\title{
Gesellschaft auf einen Blick OECD-SOZIALINDIKATOREN
}

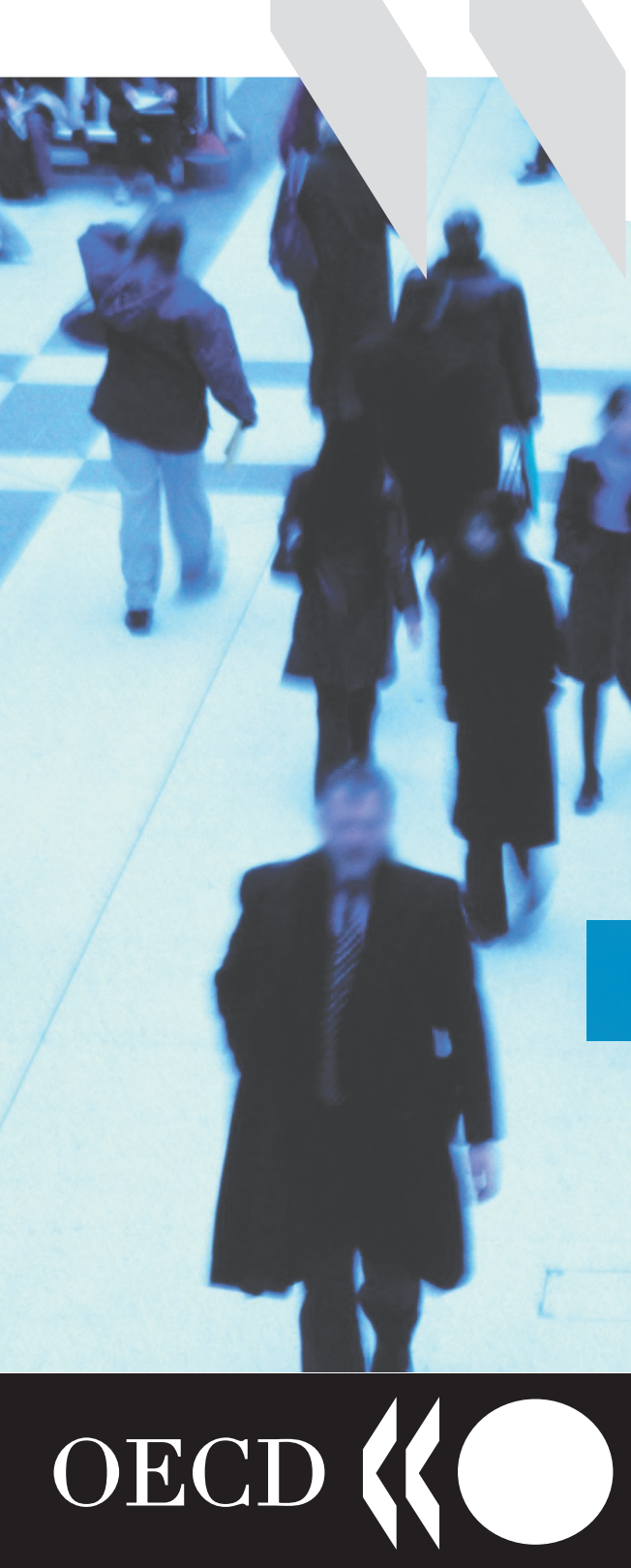

OECDPUBLISHING

2006 


\section{Gesellschaft auf einen Blick OECD-SOZIALINDIKATOREN}

Soziale Probleme haben selten eine alleinige Ursache. Geht es beispielsweise darum, Lösungen für das Problem der sozialen Ausgrenzung zu finden, muss gleichzeitig auch gegen Schranken für die Arbeitsmarktintegration, Gesundheitsprobleme und ein niedriges Bildungsniveau vorgegangen werden. Um den Herausforderungen der alternden Gesellschaft gerecht zu werden, bedarf es neuer Konzepte für Gesundheitswesen, Beschäftigung und Renten. Sozialindikatoren ermöglichen die Betrachtung der Gesellschaft aus einer breiteren Perspektive, wie sie für alle internationalen Vergleiche und Beurteilungen sozialer Entwicklungen, Ergebnisse und Maßnahmen notwendig ist. Durch die Verknüpfung von Status- und Reaktionsindikatoren über ein breites Spektrum an Politikfeldern gestatten Sozialindikatoren es dem Leser, sich darüber zu informieren, ob und wie die Maßnahmen und gesellschaftlichen Aktionen von ihrer allgemeinen Stoßrichtung her wesentlichen sozialen Problemen Rechnung tragen, denen sich die Gesellschaften der OECD-Länder gegenübersehen. Sozialindikatoren bieten einen knappen und präzisen Überblick über gesellschaftliche Trendentwicklungen und die einschlägigen Maßnahmen, schenken gleichzeitig aber auch dem unterschiedlichen nationalen Kontext, in dem diese Politiken verfolgt werden, gebührende Beachtung. Die Sozialindikatoren in Gesellschaft auf einen Blick lassen sich anhand einer zweidimensionalen Klassifikation darstellen. Die erste Dimension entspricht den drei Hauptzielen der Sozialpolitik - Autonomie, soziale Gerechtigkeit und sozialer Zusammenhalt. Die zweite Dimension bezieht sich auf die Natur der Indikatoren - den sozialen Kontext, den sozialen Status und die Reaktion der Gesellschaft. Die vorliegende Ausgabe bietet ferner ein breites Spektrum an Informationen über gesellschaftliche Aspekte wie demographische Entwicklung, Familienmerkmale, Beschäftigung, erwerbstätige Mütter, Lohnersatzquoten bei Nichterwerbstätigkeit, Armutspersistenz, Sozialausgaben, Gesundheitsausgaben, subjektives Wohlbefinden und Suizide. Ferner enthält der Bericht einen Interpretationsleitfaden für den Leser zum besseren Verständnis des Aufbaus der OECD-Sozialindikatoren sowie den Versuch einer Beurteilung der Rolle der Sozialindikatoren innerhalb des umfassenderen Programms zur Messung des Wohlergehens der Bürger und Gesellschaften des OECD-Raums.

www.oecd.org/els/social/indicators/SAG

Die Bücher, periodisch erscheinenden Publikationen und statistischen Daten der OECD sind nunmehr in unserer Online-Bibliothek unter www.SourceOECD.org erhältlich.

Diese Veröffentlichung ist im Rahmen folgender thematischer Abonnements über SourceOECD verfügbar:

Social Issues/Migration/Health

Wegen näherer Einzelheiten bezüglich des Online-Zugangs zu OECD-Veröffentlichungen wenden Sie sich bitte an Ihre Informations- und Dokumentationsstelle oder schreiben Sie uns an

SourceOECD@oecd.org

www.oecd.org 


\title{
Gesellschaft auf einen Blick
}

OECD-SOZIALINDIKATOREN

\author{
2006
}




\section{ORGANISATION FÜR WIRTSCHAFTLICHE ZUSAMMENARBEIT UND ENTWICKLUNG}

Die OECD ist ein in seiner Art einzigartiges Forum, in dem die Regierungen von 30 demokratischen Staaten gemeinsam daran arbeiten, den gl obalisierungsbedingten Herausforderungen im Wirtschafts-, Sozial- und Umweltbereich zu begegnen. Die OECD steht auch in vorderster Linie bei den Bemühungen um ein besseres Verständnis der neuen Entwicklungen und der dadurch ausgelösten Befürchtungen. Sie hilft den Regierungen dabei, diesen neuen Gegebenheiten Rechnung zu tragen, indem sie Untersuchungen zu Themen wie Corporate Governance, Informationswirtschaft oder Probleme der Bevölkerungsalterung durchführt. Die Organisation bietet den Regierungen einen Rahmen, der es ihnen ermöglicht, ihre Politikerfahrungen auszutauschen, nach Lösungsansätzen für gemeinsame Probleme zu suchen, empfehlenswerte Praktiken aufzuzeigen und auf eine Koordinierung nationaler und internationaler Politiken hinzuarbeiten.

Die OECD-Mitgliedstaaten sind: Australien, Belgien, Dänemark, Deutschland, Finnland, Frankreich, Griechenland, Irland, Island, Italien, Japan, Kanada, Korea, Luxemburg, Mexiko, Neuseeland, die Niederlande, Norwegen, Österreich, Polen, Portugal, Schweden, Schweiz, die Slowakische Republik, Spanien, die Tschechische Republik, Türkei, Ungarn, das Vereinigte Königreich und die Vereinigten Staaten. Die Kommission der Europäischen Gemeinschaften nimmt an den Arbeiten der OECD teil.

Über die OECD-Veröffentlichungen finden die Arbeiten der Organisation weite Verbreitung. Letztere erstrecken sich insbesondere auf Erstellung und Analyse statistischer Daten und Untersuchungen über wirtschaftliche, soziale und umweltpolitische Themen sowie die von den Mitgliedstaaten vereinbarten Übereinkommen, Leitlinien und Standards.

Das vorliegende Dokument wird unter der Verantwortung des Generalsekretärs der OECD veröffentlicht. Die darin zum Ausdruck gebrachten Meinungen und A rgumente spiegeln nicht zwangsläufig die offizielle Einstellung der Organisation oder der Regierungen ihrer Mitgliedstaaten wider.

Originalfassungen veröffentlicht unter dem Titel:

Society at a Glance - OECD Social Indicators - 2006 Edition

Panorama de la société - Les indicateurs sociaux de l'OCDE - Édition 2006

Übersetzung durch den Deutschen Übersetzungsdienst der OECD 


\section{Vorwort}

ies ist die vierte Ausgabe von Gesellschaft auf einen Blick, dem im zweijährigen Turnus erscheinenden Kompendium von Sozialindikatoren der OECD, das dieses Jahr erstmals auf Deutsch erscheint. Dieser Bericht soll der wachsenden Nachfrage nach quantitativen Daten zur Beantw ortung der Frage gerecht werden, ob in unseren Gesellschaften die Ungleichheiten größer oder kleiner werden, ob sich der Gesundheitszustand der Menschen verbessert und ob der soziale Zusammenhalt zu- oder abnimmt. In der Ausgabe 2006 werden einige in den ersten drei Ausgaben enthaltene Indikatoren aktualisiert und neue hinzugefügt: zu den Kosten der Kinderbetreuung, zur A rmutspersistenz, zur gesundheitlichen Ungleichheit und zum Vertrauen in die politischen Institutionen. Der Bericht enthält auch zwei neue Sonderkapitel: a) einen Interpretationsleitfaden, der den Lesern das Verständnis des Aufbaus der OECD-Sozialindikatoren erleichtern soll, und b) den Versuch einer Beurteilung der Rolle der Sozialindikatoren innerhalb des umfassenderen Programms zur Messung des Wohlergehens der Bürger und Gesellschaften des OECD-Raums. Detailliertere Informationen zu sämtlichen Indikatoren, auch solchen, die in dieser Ausgabe nicht enthalten sind, finden sich auf der entsprechenden OECD-Website (www.oecd.org/els/social/indicators/sag).

Dieser Bericht wurde von Anna Cristina D'Addio, Pauline Fron, Maxime Ladaique und Marco Mira d'Ercole erstellt. Da er ein breites Spektrum von Themen anspricht, wäre er nicht möglich gewesen ohne die Mitwirkung zahlreicher Personen in- und außerhalb der OECD-Abteilung Sozialpolitik, darunter Willem Adema, Gaëlle Balestat, Herwig Immervoll, Insook Jeong, Michael Förster, Rie Fujisawa, Gaetan Lafortune, David Morgan, Pascal Marianna, Christopher Prinz, Monika Queisser, Peter W hiteford und Ed W hitehouse. M ark Pearson, Leiter der OECD-A bteilung Sozialpolitik, spielte die führende Rolle bei der Ausarbeitung des Projektes und übernahm dessen Oberaufsicht. 


\section{Dieser Bericht enthält ...}

\section{StatLinks 部 SL}

Ein Service für OECD-Veröffentlichungen, der es ermöglicht, Dateien im Excel-Format herunterzuladen.

Suchen Sie die StatLinks rechts unter den in diesem Bericht wiedergegebenen Tabellen oder Abbildungen. Um die entsprechende Datei im Excel-Format herunterzuladen, genügt es,

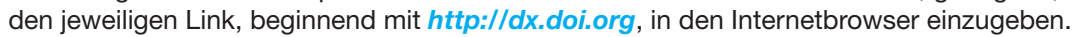
Wenn Sie die elektronische PDF-Version online lesen, dann brauchen Sie nur den Link anzuklicken. Sie finden StatLinks in weiteren OECD-Publikationen. 


\section{Inhaltsverzeichnis}

Teil I

Struktur und Definition der Indikatoren

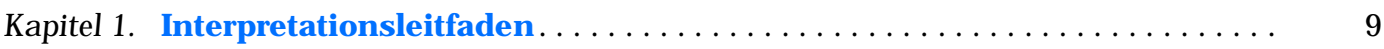

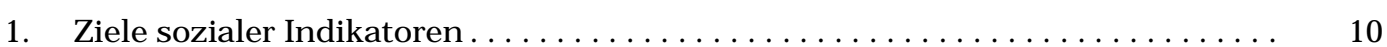

2. Der Rahmen der OECD-Sozialindikatoren $\ldots \ldots \ldots \ldots \ldots \ldots \ldots \ldots \ldots \ldots \ldots \ldots$

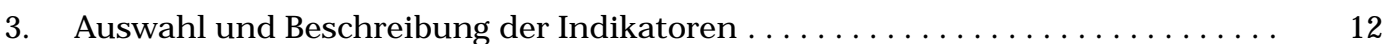

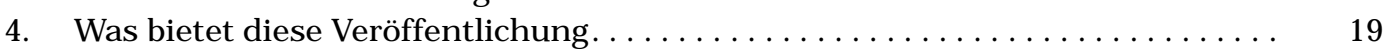

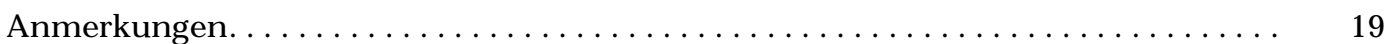

Kapitel 2. Die Messung des Wohlergehens:

Welche Rolle spielen Sozialindikatoren? . . . . . . . . . . . . . 21

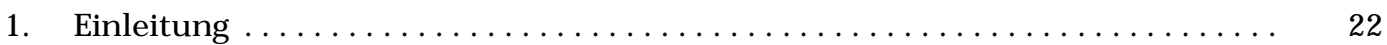

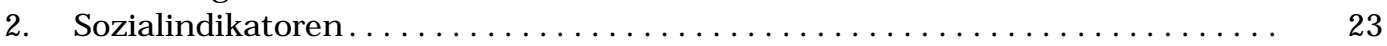

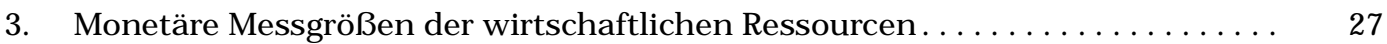

4. Weitere Berichtigungen der Messgrößen auf der Basis

der Volkswirtschaftlichen Gesamtrechnung . . . . . . . . . . . . . . . . . . 31

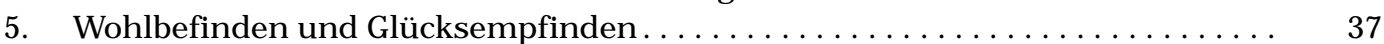

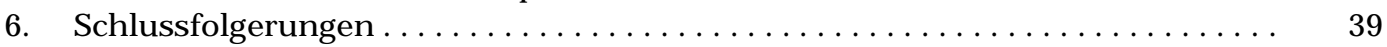

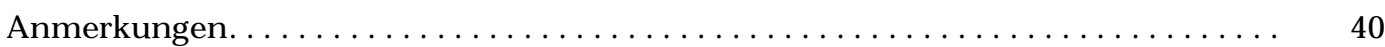

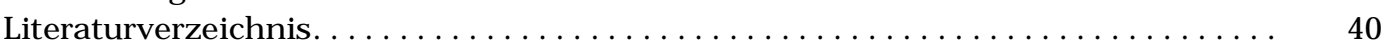

Teil II

OECD-Sozialindikatoren

Allgemeine Kontextindikatoren (GE):

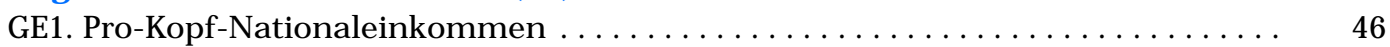

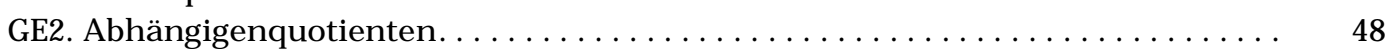

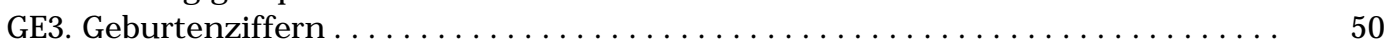

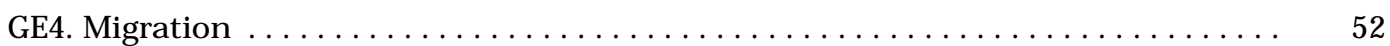

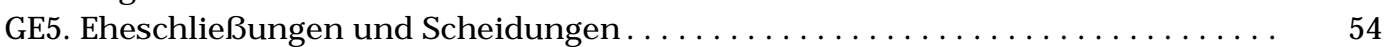

Autonomie-Indikatoren (SS):

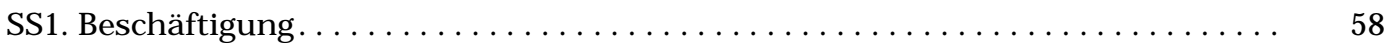

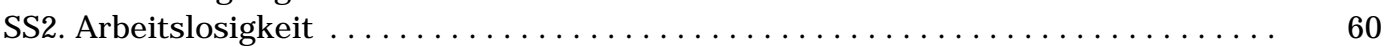

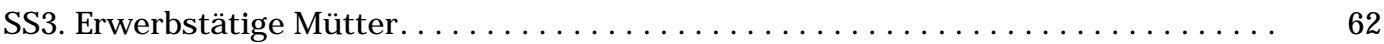

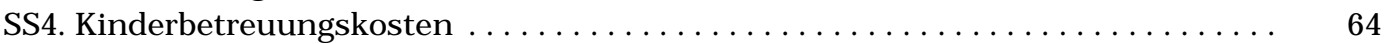

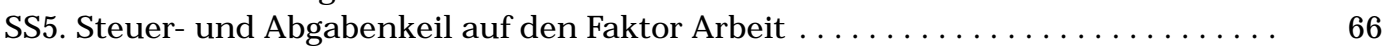

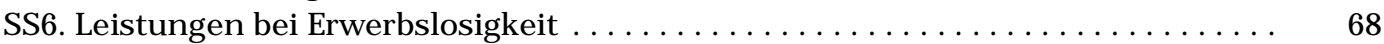

SS7. Schülerleistungen $\ldots \ldots \ldots \ldots \ldots \ldots \ldots \ldots \ldots \ldots \ldots \ldots \ldots \ldots \ldots \ldots \ldots \ldots \ldots \ldots \ldots \ldots \ldots, \quad 70$

Indikatoren der sozialen Gerechtigkeit (EQ):
EQ1. Materielle Entbehrung $\ldots \ldots \ldots \ldots \ldots \ldots \ldots \ldots \ldots \ldots \ldots \ldots \ldots \ldots \ldots \ldots \ldots \ldots$

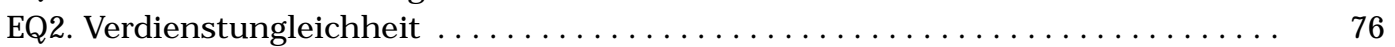




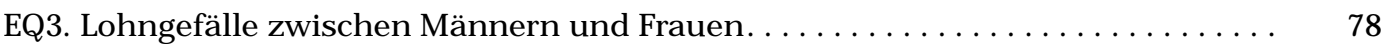

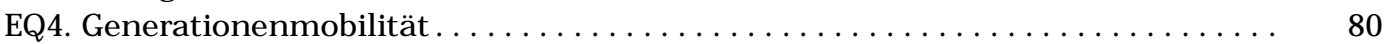

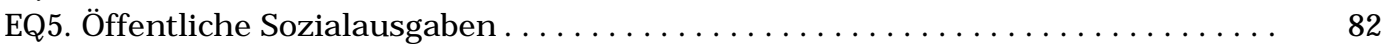

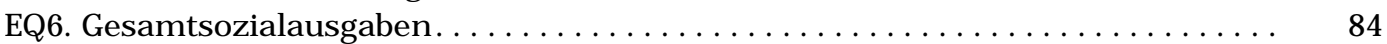

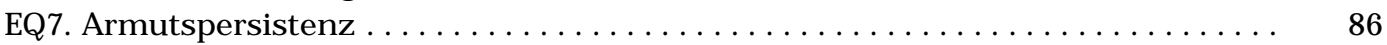

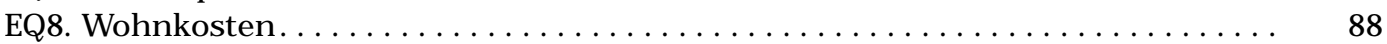

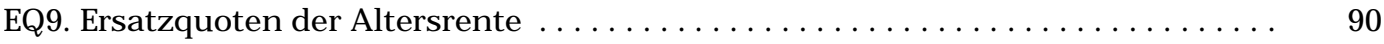

Gesundheitsindikatoren (HE):

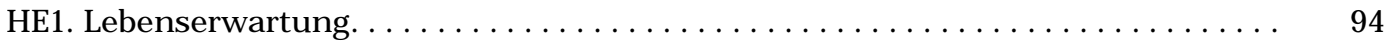

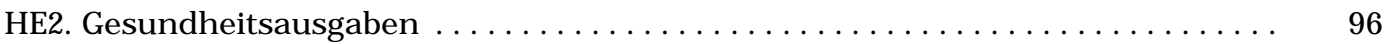

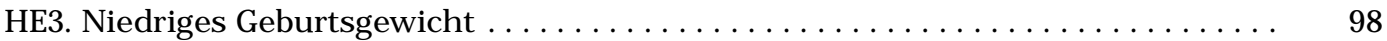

HE4. Krankheitsbedingte Abwesenheit vom Arbeitsplatz . . . . . . . . . . . 100

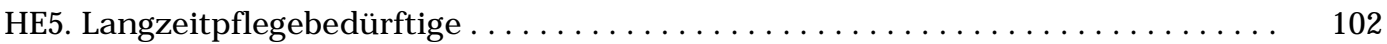

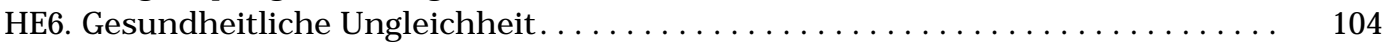

Indikatoren für den sozialen Zusammenhalt (CO):

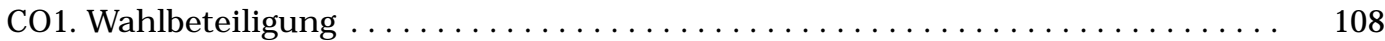

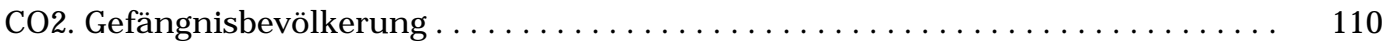

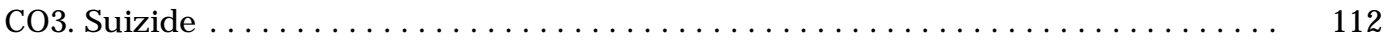

C04. Arbeitsunfälle ..................................... 114

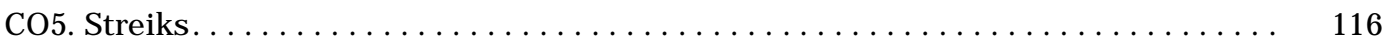

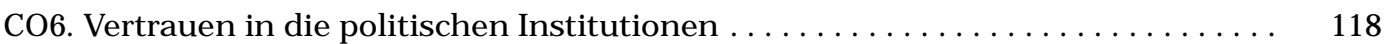

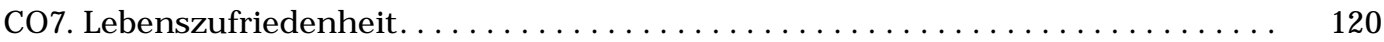


TEIL I

\section{Struktur und Definition der Indikatoren}





\section{TEIL I}

Kapitel 1

\section{Interpretationsleitfaden}




\section{Ziele sozialer Indikatoren}

Mit dem vorliegenden Bericht sollen Erkenntnisse vermittelt werden, die für die Beantwortung folgender zwei Fragen bedeutsam sind:

- Welche Fortschritte haben die OECD-Länder im Hinblick auf ihre soziale Entwicklung erreicht?

- Wie wirksam waren die Maßnahmen der Gesellschaft, um die soziale Entwicklung voranzubringen?

Zur Beantwortung der ersten Frage bedarf es Indikatoren, die ein breites Spektrum sozialer Fragen abdecken. Da die soziale Entwicklung Gesundheit, Bildung, Wirtschaftsressourcen und eine stabile Grundlage für soziale Interaktionen voraussetzt, müssen die Indikatoren Informationen zu all diesen Dimensionen liefern. Die zweite Frage lässt sich schwerer beantworten. Gesellschaften versuchen generell über die staatliche Politik Ergebnisse im Sozialbereich zu beeinflussen, und es stellt sich die Frage, ob diese staatlichen Maßnahmen ihre Ziele effektiv erreichen. Indikatoren helfen bei der Beurteilung dieser Frage. Ein erster Schritt besteht darin, die Veränderungen im Sozialbereich, die die Sozialpolitik zu bewirken sucht, mit der Skala der Ressourcen zu vergleichen, die für diesen Zweck eingesetzt werden. Auch wenn ein derartiger Vergleich keine umfassende Evaluierung der Effektivität eines spezifischen Programms ermöglicht, können die Indikatoren dennoch jene Bereiche hervorheben, in denen es einer eingehenderen Analyse bedarf.

\section{Der Rahmen der OECD-Sozialindikatoren}

Auch wenn die in dieser Ausgabe gewählte Struktur keinen umfassenden Rahmen für Sozialstatistiken bietet, handelt es sich dennoch um mehr als eine einfache eindimensionale Auflistung. Sie orientiert sich an den in anderen OECD-Tätigkeitsfeldern gesammelten Erfahrungen und Informationen im Hinblick auf die Beurteilung der Politikmaßnahmen und Ergebnisse, die mit diesen Maßnahmen in einer Vielzahl von Bereichen angestrebt werden. Diese Struktur macht sich insbesondere die von der OECD gesammelten Erfahrungen mit Umweltindikatoren zu Nutze. Diese Indikatoren sind in einen als PSR-Modell (Pressure-State-Response) bekannten Rahmen integriert ${ }^{1}$. Nach diesem Modell üben menschliche Aktivitäten Druck (pressure) auf die Umwelt aus, der seinerseits die Naturressourcen und den Umweltzustand (state) beeinflusst und die Gesellschaft dazu bewegt, auf diese Veränderungen mittels verschiedener Maßnahmen zu reagieren (societal response). Der PSR-Rahmen ermöglicht die Hervorhebung dieser Verknüpfungen und hilft Entscheidungsträgern und der breiten Öffentlichkeit, die Zusammenhänge zwischen Umwelt- und sonstigen Problemen besser zu erkennen. In diesem Rahmen wurden die Indikatoren für die Reaktion von Staat und Gesellschaft (Reaktionsindikatoren) und die Indikatoren der Faktoren, die sie zu beeinflussen suchen (Zustands- und Belastungsindikatoren), miteinander verknüpft. 
Ein ähnliches Konzept findet im vorliegenden Bericht für die Sozialindikatoren Anwendung. Die Indikatoren werden anhand von zwei Dimensionen klassifiziert, von denen die erste die Natur dieser Indikatoren betrachtet und sie wiederum in drei Bereiche unterteilt ${ }^{2}$ :

1. Die Indikatoren für den Sozialen Kontext beziehen sich auf Variablen, die, ohne ein unmittelbares Ziel der Sozialpolitik zu sein, für das Verständnis des allgemeinen Kontextes, in dem die Sozialpolitik operiert, entscheidend sind. So bildet beispielsweise der Anteil älterer Menschen an der Gesamtbevölkerung kein unmittelbares Politikziel, ist aber ein Bestimmungsfaktor dafür, wie sich einzelne Maßnahmen auf den Lebensstandard älterer Menschen auswirken und wie hoch die damit verbundenen Kosten sind. Im Unterschied zu anderen Indikatoren können sozialpolitische Indikatoren nicht eindeutig als "gut" oder "schlecht" interpretiert werden. Beispiel sweise können die zwischen den Ländern bestehenden Unterschiede in Bezug auf die Zahl der Alleinerzieherhaushalte auf kulturelle Faktoren zurückzuführen sein, obgleich in allen Ländern die für die Sozialpolitik Verantwortlichen aufgerufen sind, sich mit den Folgen auseinanderzusetzen.

2. Die Indikatoren für den Sozialen Status beschreiben jene Ergebnisse im Sozial bereich, die Politikmaßnahmen zu beeinflussen suchen. Im Idealfall werden Indikatoren gewählt, die einfach und eindeutig interpretiert werden können - so hätten beispielsweise alle Länder lieber niedrige als hohe Armutsquoten. Diese Indikatoren beschreiben die allgemeinen sozialen Bedingungen der Bevölkerung oder eine spezifische Dimension, auf die die Sozialpolitik Einfluss auszuüben versucht.

3. Die Indikatoren für die Reaktion der Gesellschaft liefern Informationen über die Größenordnung und die Natur sozialpolitischer Interventionen, d.h. über die konkreten Maßnahmen, die die Gesellschaft zur Beeinflussung des sozialen Status ergreift. Sie umfassen Indikatoren für den Kurs der staatlichen Politik, aber auch für die Aktivitäten des privaten Sektors und einer Reihe von Nichtregierungsorganisationen (NRO). In diese Kategorie fallen Indikatoren für die Entwicklung privater Altersversorgungssysteme oder die von Einzelnen bzw. Familien übernommene Verantwortung für ältere Menschen und Kinder ${ }^{3}$. Ein Vergleich der Reaktionsindikatoren mit den Statusindikatoren gibt erstklassigen Aufschluss über die Effizienz der staatlichen Politik.

Die zweite Dimension des OECD-Rahmens gruppiert die Indikatoren anhand der von ihnen abgedeckten globalen Politikfelder. Zur Klassifizierung der Indikatoren des sozialen Status und der Reaktionen der Gesellschaft werden vier Ziele der Sozialpolitik aufgestellt:

A) Die Stärkung der Autonomie ist ein grundlegendes Ziel der Sozialpolitik, das auch im Kommuniqué der OECD-Tagung der Sozial- und Gesundheitsminister (www.oecd.org/ socmin2005) ausdrücklich formuliert wurde. Erhöht wird die Autonomie des Einzelnen, indem seine aktive Teilnahme am Wirtschafts- und Gesellschaftsleben wie auch Selbstständigkeit in Aktivitäten des täglichen Lebens gefördert wird.

B) Soziale Gerechtigkeit bezieht sich in diesem Zusammenhang auf Benachteiligungen am Arbeitsmarkt und in der Gesellschaft sowie auf die Chancengleichheit. Gerechte Ergebnisse werden vor allem anhand des Zugangs von Haushalten zu Ressourcen gemessen.

C) Zwar ist die Verbesserung des Gesundheitszustand der Bevölkerungen das grundlegende Ziel aller Gesundheitssysteme, doch geht die Fokussierung der für die Erreichung dieses Ziels zu berücksichtigenden Faktoren über die alleinige Betrachtung 
von Krankheiten und Heilmethoden hinaus und umfasst auch andere soziale Faktoren wie Mortalität und Morbidität.

D) Der soziale Zusammenhalt wird häufig als überwölbendes Ziel der Sozialpolitik eines Landes identifiziert. Während wenig Übereinstimmung hinsichtlich der genauen Bedeutung dieses Begriffs besteht, gibt es dennoch eine Reihe pathologischer Befunde, die Aufschluss über einen Mangel an sozialem Zusammenhalt liefern. Hierzu zählen beispielsweise Kriminalität, Inhaftierungen, Selbstmorde, Arbeitskämpfe und familiäre Instabilität. Ferner fallen unter diese Kategorie auch Messungen des Beteiligungsgrads des Einzelnen am Gemeinschaftsleben.

Auf der Basis dieser beiden Dimensionen lassen sich die OECD-Sozialindikatoren als eine "Matrix" darstellen (Tabelle 1.1).

\section{Auswahl und Beschreibung der Indikatoren}

Die statistische Datenerfassung der OECD-Länder weicht vor allem im Sozialbereich erheblich voneinander ab. Bei der Auswahl der Indikatoren im Hinblick auf ihre Aufnahme in diesen Bericht wurden folgende Überlegungen angestellt:

- Eine erste Erwägung betrifft das Ausmaß der Vergleichbarkeit der Indikatoren zwischen den Ländern. Wenngleich in dieser Ausgabe angestrebt wurde, für jeden untersuchten Bereich die am besten vergleichbaren Informationen zu liefern, beschränken sich die hier dargelegten Indikatoren nicht auf jene mit "absoluter" Vergleichbarkeit; der Leser wird aber auf die Natur der verwendeten Daten und die jeweils mit innen verbundene Problematik aufmerksam gemacht.

- Eine zweite Erwägung berührt die Frage, ob Indikatoren aufgenommen werden sollen, die für alle Länder verfügbar sind, oder umgekehrt, wie weit von diesem Grundsatz abgewichen werden kann. Generell gilt, dass diese Ausgabe nur jene Indikatoren enthält, die in der Mehrzahl der OECD-Länder verfügbar sind.

- Eine dritte Überlegung bezieht sich auf die sinnvolle Aufschlüsselung der Daten. Sozialindikatoren können häufig in Unterkategorien aufgeteilt werden, wie beispielsweise Alter der Personen, Familienstand und Geschlecht. Die verfügbare Aufschlüsselung (entweder nach individuellen oder Haushaltsmerkmalen) ist von Indikator zu Indikator unterschiedlich, wobei in diesem Bericht mehrere verwendet werden. Es wird auch nicht der Versuch unternommen, alle Daten in derselben Einheit darzustellen, d.h. die in dieser Ausgabe zu Grunde gelegten Sozialindikatoren stellen einen Mix aus absoluten Zahlen, Währungseinheiten, BIP-Prozentsätzen usw. dar.

Für jeden ausgewähIten Indikator enthält Teil II dieses Berichts eine Beschreibung der Hauptmerkmale gemeinsam mit allgemeinen Informationen zu Definition und Messung. Die meisten Indikatoren existieren bereits in der einen oder anderen Form, und viele werden auf regelmäßiger Basis in anderen OECD-Veröffentlichungen publiziert (z.B. Labour Force Statistics, Social Expenditure Database, Gesundheitsdaten); andere wurden auf Ad-hoc-Basis zusammengetragen. Während einige Indikatoren in alle Ausgaben von Gesellschaft auf einem Blick (vor 2006 nur in englischer Originalfassung mit dem Titel Society at a Glance) aufgenommen wurden, erscheinen andere nur von Fall zu Fall.

Einzelne Indikatoren können für multiple Bereiche der Sozialpolitik von Bedeutung sein, d.h. sie können mehr als einer Kategorie zugeordnet werden. Beispielsweise ist die Fähigkeit, Aktivitäten des täglichen Lebens ohne Hilfe durchzuführen, ein Indikator für sozialen Zusammenhalt, Autonomie und Gesundheit. Auch wenn dieses Problem kein 


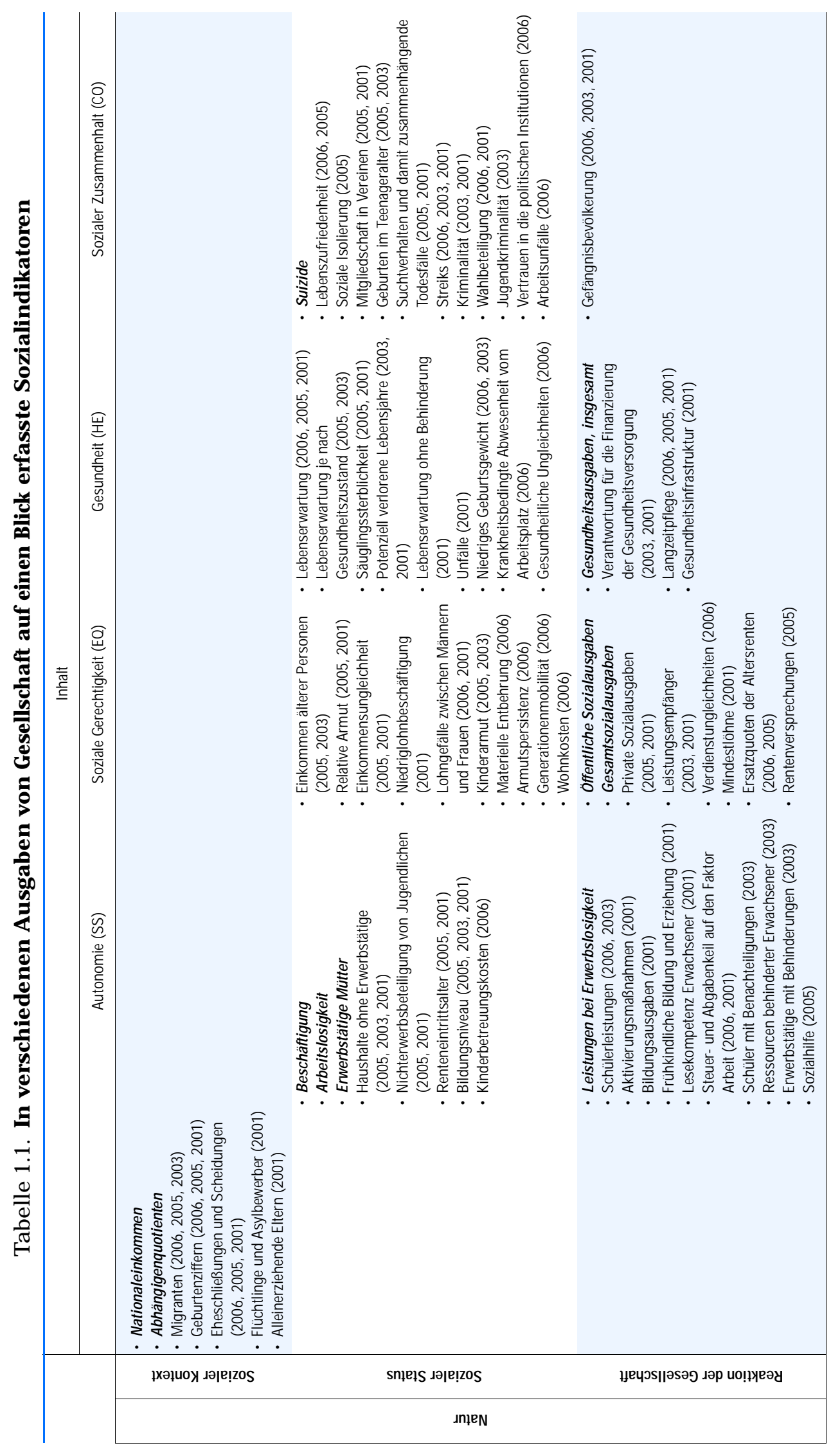

$\stackrel{0}{\frac{0}{0}}$

๑

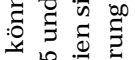

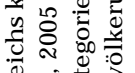

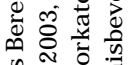

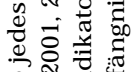

잉

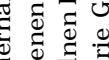

号

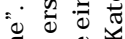

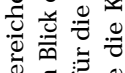

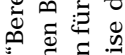

ब के

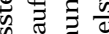

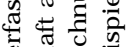

通这

这

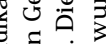

类

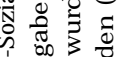

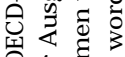

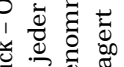

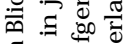

을

言的曾产

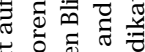

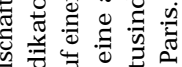

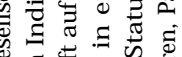

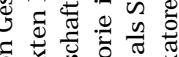

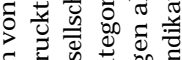

政密

号

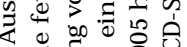

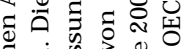

屯

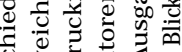

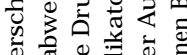

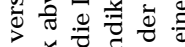

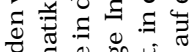

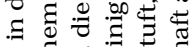

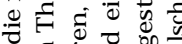

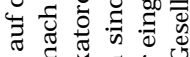

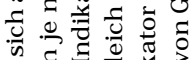

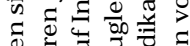

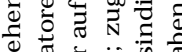

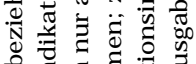

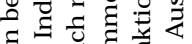

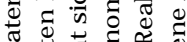

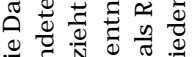

-

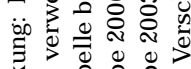

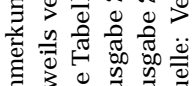

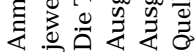


spezifisches Problem der Sozialpolitik an sich ist, wurde in dieser Ausgabe die Lösung gewählt, die Indikatoren nur in der Kategorie aufzuführen, für die sie am unmittelbarsten von Bedeutung sind, statt sie in verschiedenen Abschnitten zu wiederholen. Gleichzeitig enthalten aber die Beiträge zu diesem Bericht häufig mehrere Indikatoren, die Informationen sowohl über den sozialen Status als auch die Reaktion der Gesellschaft liefern. Im gesamten Bericht dient der in Klammern stehende Code hinter jedem einzelnen Indikator (z.B. GE1) der Zuordnung desselben zu einem Politikfeld (wie in den unten stehenden Tabellen aufgeführt), und die Nummerierung der Indikatoren dient der Vereinfachung von Querverweisen. Obwohl Bezeichnung und Code der in dieser Ausgabe erfassten Indikatoren von denen in früheren Ausgaben von Gesellschaft auf einen Blick abweichen können, werden Anstrengungen unternommen, um in den untersuchten Politikfeldern Kontinuität zu gewährleisten.

\subsection{Kontextindikatoren (GE)}

Beim Vergleich der Indikatoren für den sozialen Status und die Reaktion der Gesellschaft kann leicht die Aussage getroffen werden, dass ein Land im Vergleich zu anderen schlecht abschneidet oder dass wieder ein anderes Land im Vergleich zu anderen sehr viel Geld für ein spezifisches Politikziel ausgibt. Wichtig ist es, derartige Aussagen in einen globaleren Kontext zu stellen. So ist beispielsweise das Volkseinkommen in den OECD-Ländern unterschiedlich. Besteht also ein Zusammenhang zwischen Nationaleinkommen und Gesundheit, steht zu erwarten, dass die gesundheitlichen Bedingungen in reicheren Ländern unabhängig von der Reaktion der Gesellschaft besser sind als in ärmeren. Falls die Nachfrage nach Gesundheitsleistungen mit dem Einkommen steigt (was der Fall zu sein scheint), müssten reichere Länder (in Prozent des Nationaleinkommens) mehr Geld für die Gesundheitsversorgung aufwenden als ärmere. Das bedeutet aber nicht, dass die Indikatoren für den Gesundheitszustand und die Gesundheitsausgaben irreführend sind: Es bedeutet nur, dass bei der Interpretation der Indikatoren der den Daten zu Grunde liegende allgemeine Kontext berücksichtigt werden sollte. Ein weiteres Merkmal der meisten Kontextindikatoren besteht darin, dass a priori keine Aussage darüber möglich ist, ob ein hoher Wert positiv oder negativ ist.

Viele Kontextindikatoren sind für die Interpretation mehrerer in dieser Veröffentlichung enthaltener Indikatoren von Bedeutung. Hierzu zählen das Pro-KopfNationaleinkommen (GE1), das Auswirkung auf Qualität, Quantität und Natur des sozialen Schutzes hat, den eine Gesellschaft bieten kann, aber auch auf den Abhängigenquotienten (GE2), Geburtenziffern (GE3), Migration (GE4) sowie die Kategorie Eheschließungen und Scheidungen (GE5). Wie weiter oben festgehalten wurde, werden Kontextindikatoren keinem der vier Felder der Sozialpolitik zugeordnet - soziale Gerechtigkeit, Autonomie, Gesundheit oder sozialer Zusammenhalt.

\section{Liste der allgemeinen Kontextindikatoren (GE)}

$\begin{array}{ll}\text { GE1. } & \text { Pro-Kopf-Nationaleinkommen } \\ \text { GE2. } & \text { Abhängigenquotienten } \\ \text { GE3. } & \text { Geburtenziffern } \\ \text { GE4. } & \text { Migration } \\ \text { GE5. } & \text { Eneschließungen und Scheidungen }\end{array}$




\subsection{Autonomie (SS)}

Bezahlte Beschäftigung (SS1) verschafft dem Großteil der Bevölkerung im erwerbsfähigen Alter Zugang zu wirtschaftlichen Ressourcen, verhilft ihm zu einer Identität, ermöglicht gesellschaftliche Interaktionen und verleiht ihm einen Status. Darüber hinaus stützen sich fast alle sozialen Sicherungssysteme zur Finanzierung ihrer Leistungen auf Beiträge der Erwerbsbevölkerung. So ist auch die Förderung einer höheren Beschäftigung für alle OECD-Länder eine Priorität.

Dennoch hat Arbeitslosigkeit (SS2) häufig zur Folge, dass die Versorgung des Einzelnen und seiner Familie durch den Erwerb von Arbeitseinkommen manchmal keine Realität ist. Der Zugang zu bezahlter Beschäftigung ist für Mütter mit kleinen Kindern häufig ganz besonders schwierig (SS3), was in vielen Fällen auf die hohen Kosten der Kinderbetreuung zurückzuführen ist (SS4). Da die Benachteiligungen am Arbeitsmarkt in erster Linie gering qualifizierte Arbeitskräfte betreffen, dürften Leistungsunterschiede am Ende der Pflichtschulzeit dauerhafte Auswirkungen auf die Chancen der Schüler für einen erfolgreichen Übergang von der Schule ins Erwerbsleben haben (SS7).

Die Antwort der Gesellschaft auf dieses Problem besteht traditionsgemäß aus einer Kombination von finanziellen Unterstützungsleistungen für Personen, die nicht in der Lage sind, für sich aufzukommen, und Maßnahmen zur Überwindung der Hindernisse für die Ausübung einer Erwerbstätigkeit sowie zur Erleichterung der Integration in den Arbeitsmarkt. Weisen diese beiden Arten von Maßnahmen aber konzeptuelle Schwächen auf, so kann ihre Wirkung antinomisch sein. Insbesondere können Arbeitslosen zur Verfügung gestellte Sozialleistungen den finanziellen Anreiz zur Aufnahme einer Beschäftigung (SS6) und auch die Nachfrage der Unternehmen nach Arbeitskräften verringern (SS5).

Die nachstehende Tabelle enthält eine Auflistung der Indikatoren des sozialen Status und der Reaktion der Gesellschaft, die für die Beurteilung der Frage, ob OECD-Länder die Ziele zur Gewährleistung der Autonomie des Einzelnen und seiner Familie erreicht haben, besonders relevant sind.

\section{Liste der Autonomie-Indikatoren (SS) ${ }^{1}$}

\begin{tabular}{l|l}
\hline Sozialer Status & Reaktion der Gesellschaft \\
\hline SS1. Beschäftigung & SS5. Steuer- und Abgabenkeil auf den Faktor Arbeit \\
SS2. Arbeitslosigkeit & SS6. Leistungen bei Erwerbslosigkeit \\
SS3. Erwerbstätige Mütter & \\
SS4. Kinderbetreuungskosten & \\
SS7. Schülerleistungen & EQ5. Öfentliche Sozialausgaben \\
EQR. Verdienstungleichheit & EQ6. Gesamtsozialausgaben \\
EQB. Lohngefällezwischen Männern und Frauen & \\
EQ4. Generationenmobilität & \\
EQ7. Armutspersistenz & \\
\hline
\end{tabular}

1. Kursiv gedruckt sind jene Indikatoren, die trotz ihres Erscheinens in einer anderen Unterkategorie auch für die Beurteilung der Autonomie relevant sind.

\subsection{Soziale Gerechtigkeit (EQ)}

Dieser Begriff hat zahlreiche Dimensionen und kann sich u.a. auf den Zugang zu sozialen Diensten, aber auch auf wirtschaftliche Chancen bzw. Ergebnisse beziehen. Die 
Auffassungen darüber, was unter einer gerechten Verteilung der Ressourcen oder Chancen genau zu verstehen ist, gehen weit auseinander, und zwar sowohl auf nationaler als auch auf internationaler Ebene. Da es schwierig ist, Informationen über sämtliche Aspekte der sozialen Gerechtigkeit zu erhalten, beschränkt sich die Mehrzahl der Indikatoren des sozialen Status, die für die Beurteilung der Ergebnisse im Bereich der sozialen Gerechtigkeit relevant sind, auf Ungleichheiten bei den finanziellen Ressourcen und sehr viel seltener bei den Konsumstrukturen.

Obwohl Armut meistens anhand der finanziellen Ressourcen beurteilt wird, lässt sie sich auch über das Ausmaß der materiellen Entbehrung in den einzelnen Ländern messen (EQ1). Die Effekte der Armut hängen von der Armutspersistenz (EQ7) wie auch davon ab, ob die Armut Möglichkeiten der Generationenmobilität untergräbt (EQ4). Armut hat ihre Wurzeln häufig in größerer Verdienstungleichheit (EQ2) wie auch im Lohngefälle zwischen Männern und Frauen (EQ3), während hohe Wohnkosten die finanziellen Folgen erschweren können (EQ8).

Soziale Schutzsysteme sind das wichtigste Instrument, mit dessen Hilfe die staatliche Politik auf Probleme der sozialen Ungleichheit reagiert. Alle OECD-Länder haben soziale Schutzsysteme entwickelt (oder sind dabei, es zu tun), die es in unterschiedlichem Ausmaß erlauben, Ressourcen innerhalb der Gesellschaft umzuverteilen und deren Mitglieder gegen diverse Risiken abzusichern. Bei den Interventionen handelt es sich um Sozialleistungen, die über das soziale Sicherungssystem (EQ5) oder eine Kombination aus Steuervergünstigungen und privaten Ausgaben vergeben werden (EQ6). In den meisten OECD-Ländern dient der Großteil dieser Ressourcen der Sicherung der Renteneinkommen, und Indikatoren der von den Altersrenten (EQ9) gebotenen Lohnersatzquoten zeigen den langfristigen Effekt der heutigen Rentenbestimmungen und -parameter auf die Rentner von morgen.

Die Indikatoren der sozialen Gerechtigkeit können nicht einfach von den AutonomieIndikatoren getrennt werden. Zusammen betrachtet zeigen sie, wie sich die nationalen sozialen Sicherungssysteme mit einem chronischen politischen Dilemma auseinandersetzen, dem Interessenkonflikt zwischen der Bereitstellung angemessener Ressourcen auf der einen und der Nachhaltigkeit des Systems und Förderung der Autonomie des Einzelnen auf der anderen Seite.

\section{Liste der Indikatoren der sozialen Gerechtigkeit (EQ) ${ }^{1}$}

\begin{tabular}{l|l}
\hline Sozialer Status & Reaktion der Gesellschaft \\
\hline EQ1. Materielle Entbehrung & EQ5. Öffentliche Sozialausgaben \\
EQR. Verdienstungleichheit & EQ6. Gesamtsozialausgaben \\
EQB. Lohngefälle zwischen Männern und Frauen & EQ9. Essatzquoten der Altersrente \\
EQ4. Generationenmobilität & \\
EQ7. Armutspersistenz & \\
EQ8. Wohnkosten & SS6. Leistungen bei Nichterwerbstätigkeit \\
SS2. Beschäftigung & HE2. Gesundheitsausgaben \\
SS3. Arbeitslosigkeit & \\
SS4. Ewwerbstätige Mütter & \\
\hline
\end{tabular}

1. Kursiv gedruckt sind jene Indikatoren, die trotz ihres Erscheinens in einer anderen Unterkategorie auch für die Beurteilung der Ergebnisse im Bereich der sozialen Gerechtigkeit relevant sind. 


\subsection{Gesundheit (HE)}

Es besteht ein enger Zusammenhang zwischen sozialen und gesundheitlichen Bedingungen. So hat denn auch der Anstieg des Lebensstandards im Verein mit dem besseren Zugang zu Gesundheitsversorgungsleistungen und den kontinuierlichen Fortschritten der medizinischen Technik zu einer signifikanten Verbesserung des Gesundheitszustands beigetragen, wie sie sich beispielsweise anhand des Indikators der Lebenserwartung messen lässt (HE1). In bedeutendem Ausmaß fanden diese Fortschritte ihren Niederschlag in einer niedrigeren Säuglingssterblichkeit und Verbesserungen bei anderen Indikatoren der Kindergesundheit (wie beispielsweise die Prävalenz von niedrigem Geburtsgewicht, HE3). Dennoch sind noch schwierige Herausforderungen zu meistern. Bei den gesundheitlichen Bedingungen gibt es zwischen und innerhalb der Länder nach wie vor große Unterschiede (HE6), die häufig die Tendenz widerspiegeln, dass Personen mit niedrigem Bildungsniveau und sozialem Status früher sterben. Unzulängliche gesundheitliche Bedingungen haben direkte Auswirkungen auf die Wirtschaftsergebnisse, wenn sie zu häufiger krankheitsbedingter Abwesenheit vom Arbeitsplatz führen (HE4).

Die Gesundheitsausgaben (HE2) sind fester Bestandteil des Politikinstrumentariums der Gesundheitssysteme zur Reaktion auf Anliegen im Zusammenhang mit den gesundheitlichen Bedingungen für die breite Bevölkerung und auch für spezifische Bevölkerungsgruppen. Ein weiterer konkreter Ausdruck dieser Reaktion ist die steigende Zahl pflegebedürftiger Menschen, die Pflegeleistungen entweder in entsprechenden Einrichtungen oder im gewohnten häuslichen Umfeld in Anspruch nehmen (HE5). Und dennoch haben Gesundheitsprobleme manchmal ihre Wurzeln in anderen sozialen Bedingungen - wie Arbeitslosigkeit, Armut, unzulängliche Wohnverhältnisse -, die miteinander verknüpft sind, aber außerhalb der Reichweite der Gesundheitspolitik liegen. Darüber hinaus hängt die Effizienz gesundheitspolitischer Interventionen häufig stärker von anderen Merkmalen des Gesundheitssystems ab als vom Ausgabenniveau selbst, wie beispielsweise ein geringer Versicherungsschutz bzw. Zuzahlungen, die den Einzelnen davon abhalten können, medizinische Hilfe zu suchen ${ }^{4}$. Ein wesentlich breiteres Spektrum an Indikatoren für die gesundheitlichen Bedingungen und Interventionen findet sich in OECD-Gesundheitsdaten und dem dazugehörigen Begleitband Gesundheit auf einen Blick, der ebenfalls im zweijährigen Turnus veröffentlicht wird.

\section{Liste der Gesundheitsindikatoren (HE) ${ }^{1}$}

\begin{tabular}{l|l}
\hline Sozialer Status & Reaktion der Gesellschaft \\
\hline HE1. Lebenserwartung & HE2. Gesundheitsausgaben \\
HE3. Niedriges Geburtsgewicht & HE5. Langzeitpflegebedürftige \\
HE4. Krankheitsbedingte Abwesenheit vom Arbeitsplatz & \\
HE6. Gesundheitliche Ungleichheit & EQ5. Öfentliche Sozialausgaben \\
EQ4. Generationenmobilität & EQ6. Gesamtsozialausgaben \\
CO4. Suizide & \\
CO5. Arbeitsunfälle & \\
\hline
\end{tabular}

1. Kursiv gedruckt sind jene Indikatoren, die trotz ihres Erscheinens in einer anderen Unterkategorie auch für die Beurteilung der Gesundheitsergebnisse relevant sind. 


\subsection{Sozialer Zusammenhalt (CO)}

Die Förderung des sozialen Zusammenhalts ist in vielen OECD-Ländern ein zentrales Ziel der Sozialpolitik. Auf Grund des Fehlens einer allgemein anerkannten Definition des Begriffs wird die Erstellung geeigneter Indikatoren erschwert. Das in dieser Ausgabe gewählte Konzept besteht darin, den sozialen Zusammenhalt anhand von Indikatoren zu beurteilen, die sowohl beschreiben, bis zu welchem Grad die Bürger am gesellschaftlichen Leben teilhaben und mit den Aktivitäten ihres täglichen Lebens zufrieden sind, als auch Informationen über verschiedene Pathologien und Bedingungen liefern, die gefährdete Kranke der Gefahr aussetzen, von der Mainstream-Gesellschaft ausgeschlossen zu werden, bzw. Aufschluss über das Ausmaß der sozialen Konflikte in einem Land geben.

Die Wahlbeteiligung (CO1) und das Maß des Vertrauens der Bürger in die politischen Institutionen ihrer Gemeinde (CO6) sind zwei wichtige Dimensionen, an denen sich ablesen lässt, wie gut der Einzelne in die Gesellschaft integriert ist und am sozialen Leben teilnimmt ${ }^{5}$. Erhebungsdaten über die subjektive Lebenszufriedenheit (CO7) sind auch wichtige "direkte" Messgrößen für das Wohlbefinden des Einzelnen und den Zusammenhalt der Gesellschaft insgesamt.

Umgekehrt umfassen die Indikatoren, die nicht nur Aufschluss über persönliche Schwierigkeiten, sondern auch über tiefer liegende Missstände in der Gesellschaft geben, Messungen der Suizidraten (CO3) und Gefängnisbevölkerung (CO2). Streikindikatoren (CO5) liefern Informationen über den Konsenscharakter des Systems der ArbeitgeberArbeitnehmer-Beziehungen, wobei eine hohe Zahl an Arbeitsunfällen (CO4) häufig Missstände bei den Sicherheitsvorkehrungen am Arbeitsplatz widerspiegelt.

Über diese Indikatoren des sozialen Status hinaus lässt sich die Existenz verschiedener Gruppen und Haushalte in der Gesellschaft, die der speziellen Gefahr der sozialen Ausgrenzung ausgesetzt sind (z.B. allein lebende Personen), auch durch Kontextindikatoren beleuchten. Letztlich sollte beachtet werden, dass es sehr viel schwieriger ist, geeignete Reaktionsindikatoren zu finden. Umgekehrt beeinflussen alle Maßnahmen, die für andere Dimensionen der Sozialpolitik von Bedeutung sind (Autonomie, soziale Gerechtigkeit und Gesundheit) ebenfalls den sozialen Zusammenhalt.

\section{Liste der Indikatoren für den sozialen Zusammenhalt (CO) ${ }^{1}$}

\begin{tabular}{l|l}
\hline Sożaler Status & Reaktion der Gesellschaft \\
\hline$\infty 1 . \quad$ Wahlbeteiligung & $\infty$ R. Gefängnisbevölkerung \\
$\infty 03 . \quad$ Suizide & \\
$\infty$ 4. Arbeitsunfälle & \\
$\infty$ 5. Streiks & \\
$\infty 06 . \quad$ Vertrauen in die politischen Institutionen & EQ5. Öfentliche Sozialausgaben \\
$\infty$ 7. Lebenszufriedenheit & EQ6. Gesamtsozialausgaben \\
SS2. Arbeitslosigkeit & HE2. Gesundheitsausgaben \\
EQ1. Materielle Entbehrung & \\
EQ7. Armutspersistenz & \\
HE1. Lebenserwartung & \\
HE4. Krankheitsbedingte Abwesenheit vom Arbeitsplatz & \\
\hline
\end{tabular}

1. Kursiv gedruckt sind jene Indikatoren, die trotz ihres Erscheinens in einer anderen Unterkategorie auch für die Beurteilung der Ergebnisse des sozialen Zusammenhalts von Bedeutung sind. 


\section{Was bietet diese Veröffentlichung}

Für jede in Teil II dieses Berichts behandelte Thematik bietet der Text die Definition des oder der einschlägigen Indikatoren sowie gegebenenfalls Informationen zu Messproblemen. Die Länder weichen in so vielerlei Hinsicht voneinander ab, dass bei einigen Indikatoren kaum Anspruch auf eine präzise Definition erhoben werden kann: Zwischen den Ländern bestehende Unterschiede im Hinblick auf die Datenqualität sind unvermeidlich. Wo dies der Fall ist, wird im Text versucht, ausdrücklich auf diesen Sachverhalt hinzuweisen. Diesem ersten Abschnitt zu "Definition und Messung" folgen eine Beschreibung der Grundtrends und länderspezifischen Unterschiede bei den verschiedenen Indikatoren und eine Erklärung über die Gründe etwaiger Unterschiede. Generell enthält jeder Abschnitt Informationen für ein Jahr und für alle OECD-Länder, für die die entsprechenden Informationen verfügbar sind, und stellt Trendentwicklungen für eine Auswahl von Ländern vor. Die entsprechenden Daten werden in Form von Abbildungen und Tabellen dargestellt, mit ausgewählten Verweisen "auf weitere Informationen" und Titel von Veröffentlichungen, denen die Indikatoren entnommen wurden.

Bei den meisten Indikatoren lassen sich die zu Grunde liegenden Daten nach Alter der Personen, Geschlecht und Familienstand aufschlüsseln. Zeitreihendaten sind nahezu immer verfügbar. Bei Verzicht auf eine außergewöhnlich umfangreiche Publikation können allerdings nicht alle Dimensionen der gesammelten Indikatoren veröffentlicht werden. Die jedem Indikator zu Grunde liegenden Daten finden sich auf der OECD-Website (www.oecd.org/els/social/indicators/sag) oder durch Eingabe bzw. Anklicken der Schaltfläche "electronic books" beim "StatLink" unten rechts an jedem Indikator (wo auch die Daten für die anderen Länder verfügbar sind).

\section{Anmerkungen}

1. Der PSR-Rahmen ist wiederum eine Variante eines Konzepts, das dem von der VN-Kommission für nachhaltige Entwicklung verwendeten "Driving force-State-Response"-Modell (DSR) und dem von der Europäischen Umweltbehörde verwendeten "Driving force-Pressure-State-Impact-Response"-Modell (DPSIR) zu Grunde liegt.

2. Diese Einteilung weicht etwas vom PSR-Modell ab. Bei den Umweltindikatoren beziehen sich die Belastungsindikatoren auf Stromgrößen (Emissionen, Abfallerzeugung und Ressourcennutzung), die den Bestand an Umweltgütern (Wasser- bzw. Luftqualität, biologische Vielfalt) verändern, während Reaktionsindikatoren sich entweder auf Strom- oder Bestandsgrößen beziehen können.

3. Während die Sozialindikatoren einer der drei oben genannten Gruppen zugeordnet werden, ist die Unterscheidung zwischen Kontext und Status nicht immer so eindeutig. Die Geburtenziffern können beispielsweise in einigen Ländern das Ziel geburtenfreundlicher Maßnahmen sein, während sie in anderen Ländern ein Indikator für den sozialpolitischen Kontext sein können. Entsprechend kann Familienzerfall in einigen Ländern als Ergebnis einer gescheiterten öffentlichen Politik betrachtet werden, während er in anderen Ländern gar kein ausdrückliches Politikthema ist. Unweigerlich sind alle Trennlinien zwischen einzelnen Indikatoren etwas willkürlich.

4. Unzureichende medizinische Dienstleistungen in einigen geographischen Regionen können auch implizit zu einer Rationierung führen, und eine bessere regionale Planung könnte Lösungen für dieses Problem bieten.

5. So erfassen diese beiden Indikatoren eine wichtige Dimension des Sozialkapitals, d.h. "der Netzwerkbeziehungen, zusammen mit gemeinsamen Normen, Wertmaßstäben und Überzeugungen, die die Zusammenarbeit in oder zwischen den Gruppen erleichtern" (OECD, 2001, Vom Wohlergehen der Nationen: Die Rolle von Sozial- und Humankapital, Paris). 

TEIL I

Kapitel 2

\section{Die Messung des Wohlergehens: Welche Rolle spielen Sozialindikatoren?}




\section{Einleitung}

Sozialindikatoren liefern Informationen über das Wohlergehen, die weit über das hinausgehen, was von den traditionellen ökonomischen Messgrößen abgeleitet werden kann ${ }^{1}$. Niveau und Veränderung des Bruttoinlandsprodukts (BIP) pro Kopf der Bevölkerung waren lange Zeit hindurch Hauptmaßstab für die Messung und den Vergleich des Lebensstandards in und zwischen den Ländern. Worum es den politischen Entscheidungsträgern wie auch den Bürgern geht, reicht indessen weit über das bloße Pro-Kopf-BIP hinaus. Sie sind in erster Linie bestrebt, das allgemeine Wohlergehen der Gesellschaft für die heutigen wie die künftigen Generationen sicherzustellen.

Aber was genau ist unter "Wohlergehen" zu verstehen? Hierauf gibt es unterschiedliche Antworten. Die Sozialindikatoren messen die erfassbaren Ergebnisse in einer Reihe von Bereichen (Gesundheit, Grundbildung, Armut), und zwar ausgehend von der Prämisse, dass ein breiter Konsens über die Bedeutung dieser Elemente besteht und die betreffenden sozialen Parameter zuverlässig und unabhängig von der subjektiven Wahrnehmung des Einzel nen gemessen werden können. Andererseits geht die wirtsch aftswissenschaftliche Literatur davon aus, dass das Wohlergehen des Einzelnen von der Befriedigung seiner Wünsche entsprechend seiner jeweiligen Präferenzen abhängt, was hauptsächlich über den Markt geschieht. Die Befriedigung der Bedürfnisse ist eine Funktion des Konsums dieser Personen; da ihr Konsum aber durch ihr Einkommen bestimmt wird, kann letzteres als Hilfsvariable für das Wohlergehen betrachtet werden, und das Einkommen wiederum kann mit Hilfe der Volkswirtschaftlichen Gesamtrechnungen zuverlässig gemessen werden.

Bis in die jüngste Zeit erschien die Wahl einer monetären Messgröße wie des Pro-KopfBIP als Indikator für das Wohlergehen der Bevölkerung durchaus sinnvoll. Denn das ProKopf-BIP liefert präzise Angaben über die Kapazität eines Landes, die materiellen Bedürfnisse seiner Einwohner zu befriedigen. Und so lange die Grundbedarfsgüter knapp sind, kann davon ausgegangen werden, dass Steigerungen des Pro-Kopf-BIP in engem Zusammenhang mit einer besseren Befriedigung der Grundbedürfnisse der Bevölkerung stehen und somit ein gesteigertes Wohlergehen widerspiegeln. Die Aussagefähigkeit des Pro-Kopf-BIP als verlässlicher Indikator für das Wohlergehen wird jedoch in dem Maße fraglicher, wie sich in den fortgeschritteneren Ländern ein Übergang von der Knappheit zum Überfluss vollzieht. Die intuitive Vermutung, dass weitere Steigerungen des Wirtschaftswachstums, wenn erst einmal ein bestimmtes Niveau der materiellen Bedürfnisbefriedigung erreicht ist, nicht mehr zwangsläufig zu äquivalenten Verbesserungen des Wohlergehens der Bürger führen, wird durch zahlreiche Untersuchungen bestätigt, in denen gezeigt wird, dass ein solches Auseinanderklaffen von Steigerung des Einkommens und Steigerung des individuellen Wohlergehens sowohl auf nationaler wie auf internationaler Ebene festzustellen ist.

Daher brauchen wir Indikatoren, die die nichtmonetären Faktoren besser erfassen. Gibt es aber einen Einzelindikator, der in allen Ländern zuverlässige Messungen erlaubt 
und als alternativer Maßstab für das Wohlergehen herangezogen werden kann? Die Antwort darauf lautet leider: nein. Das könnte dafür sprechen, am Pro-Kopf-BIP als Messgröße festzuhalten: Es kann schließlich mit einem gewissen Grad an Verlässlichkeit berechnet werden und Daten liefern, die sich gut für einen Ländervergleich eignen. Allerdings sollte hierbei die historische Dimension nicht außer Acht gelassen werden. Die Entwicklung vergleichbarer ökonomischer Indikatoren ist eine relativ neue Errungenschaft. Seit dem Zweiten Weltkrieg wurden große Anstrengungen unternommen, um harmonisierte Instrumente zur Messung des Wirtschaftswachstums zu entwickeln. Dieses Instrumentarium ist in dem Maße zunehmend komplex geworden, wie in den Volkswirtschaften eine Verlagerung von der relativ einfach zu messenden Produktion von Gütern wie Weizen und Stahl auf die Erbringung von Dienstleistungen stattgefunden hat, die schwerer zu quantifizieren ist. Beträchtliche Fortschritte wurden aber auch bei der Entwicklung eines vergleichbaren Katalogs von Sozialindikatoren insbesondere seit den achtziger Jahren erzielt, als die OECD erstmals ihre Sozialindikatoren vorstellte (OECD, 1986). Diese Anstrengungen müssen fortgesetzt werden, insbesondere durch eine verstärkte Zusammenarbeit zwischen den statistischen Ämtern der Mitgliedstaaten und internationalen Organisationen wie der OECD, die in diesem Bereich eine ganz ähnliche Rolle spielen kann, wie sie es in Bezug auf die klassischen Wirtschaftsstatistiken getan hat.

Dieses Kapitel befasst sich mit vier Konzepten zur Messung des Wohlergehens². Erstens wird aufgezeigt, welche Bedeutung den in den verschiedenen Ausgaben von Gesellschaft auf einen Blick dargestellten Sozialindikatoren für das Wohlergehen zukommt und inwieweit sie mit dem Pro-Kopf-BIP korrelieren. Zweitens wird ein Überblick über die von den Volkswirtschaftlichen Gesamtrechnungen abgeleiteten monetären Messgrößen der wirtschaftlichen Ressourcen gegeben. Drittens wird untersucht, wie diese monetären Messgrößen so angepasst werden können, dass sie auch anderen Bestimmungsfaktoren des Wohlergehens Rechnung tragen, wie namentlich Freizeit, Haushaltsgröße und Aversion gegenüber Ungleichheit. Abschließend werden subjektive Messgrößen des Glücksgefühls und der Lebenszufriedenheit untersucht, bevor die Schlussfolgerungen zusammengefasst werden.

\section{Sozialindikatoren}

Die Sozialindikatoren stellen eine Ergänzung zu den vom BIP abgeleiteten Proxyvariablen des Wohlergehens dar. In diesem Kapitel wurden vier Indikatoren für die vier in Kapitel 1 beschriebenen Bereiche (Autonomie, soziale Gerechtigkeit, Gesundheit und sozialer Zusammenhalt) gewählt ${ }^{3}$. Die Auswahl dieser Indikatoren ist zwar subjektiv, basiert aber sowohl auf ihrer Bedeutung für das soziale Wohlergehen als auch auf dem Kriterium ihrer Verfügbarkeit, damit aussagefähige Ländervergleiche möglich sind.

Liefern diese Indikatoren zusätzliche Informationen zu denen, die sich vom Pro-KopfBIP ableiten lassen? Zur Beantwortung dieser Frage werden im oberen Teil der Abbildung 2.1 die Korrelationen zwischen dem Niveau dieser 16 Sozialindikatoren und dem Pro-Kopf-BIP dargestellt. Der untere Teil der Abbildung veranschaulicht die Korrelation zwischen den durchschnittlichen jährlichen Veränderungen der beiden Variablenreihen. Die Abbildung zeigt, dass der Grad der jeweiligen Korrelation zwischen 


\section{Abbildung 2.1. Korrelationen zwischen Pro-Kopf-BIP und verschiedenen Sozialindikatoren in den OECD-Ländern}

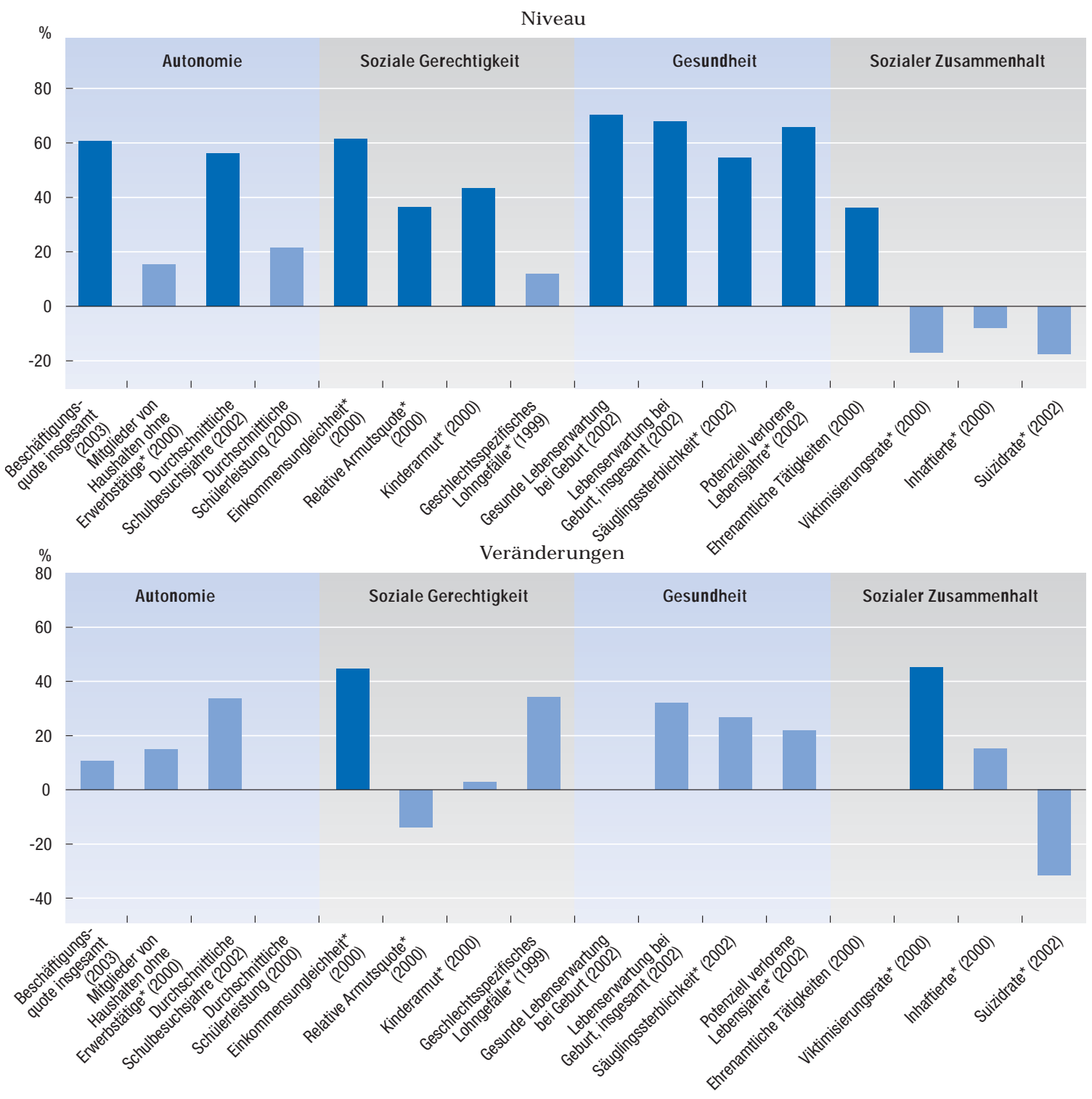

Anmerkung: Stand der Daten: um 2002; jährliche prozentuale Veränderung über den jeweils längsten belegten Zeitraum. Pearsonscher Korrelationskoeffizient: Die dunkler gefärbten Balken zeigen statistisch signifikante Korrelationen an (Niveau von 5\%). Für Variable, bei denen höhere Indikatorwerte für schlechtere Ergebnisse im Sozialbereich stehen (z.B. Säuglingssterblichkeit oder Inhaftierte, gekennzeichnet durch *), weisen die Korrelationen mit dem Pro-Kopf-Einkommen das jeweils umgekehrte Vorzeichen auf (z.B. haben Länder mit höherem Pro-Kopf-Einkommen niedrigere Säuglingssterblichkeitsraten (positives Vorzeichen) und höhere Inhaftierungsquoten (negatives Vorzeichen). Das Pro-Kopf-Einkommen wird errechnet als BIP zu laufenden Preisen und Kaufkraftparitäten, dividiert durch die Gesamtbevölkerung. Die Korrelationen beziehen sich auf die Werte des Pro-Kopf-BIP und die der Sozialindikatoren im selben Zeitraum; die Zahl der untersuchten Länder kann für die verschiedenen Variablenpaare je nach Datenverfügbarkeit variieren.

Quelle: Verschiedene Ausgaben von Gesellschaft auf einen Blick - OECD-Sozialindikatoren.

StatLink 2 http://dx.doi.org/10.1787/184757611082 
den 16 Sozialindikatoren und dem Pro-Kopf-BIP variiert, d.h. am stärksten ist er im Fall der Gesundheitsindikatoren und am schwächsten beim sozialen Zusammenhalt.

- Autonomie: Diese Größe repräsentiert den Umfang der Teilhabe des Einzelnen an Wirtschaft und Gesellschaft sowie dessen Fähigkeit, im täglichen Leben allein zurechtzukommen. Sie wird gemessen anhand der globalen Erwerbsquote, des in Haushalten ohne Erwerbstätige lebenden Bevölkerungsanteils, der durchschnittlichen Zahl der Schuljahre und der durchschnittlichen schulischen Leistungen 15-Jähriger. All diese Faktoren beeinflussen jetzt oder später die Fähigkeit des Einzelnen, seinen Lebensunterhalt angemessen zu bestreiten. Das Pro-Kopf-BIP weist eine signifikante Korrelation mit den Beschäftigungsquoten, nicht jedoch mit den Indikatoren für die Verteilung der Beschäftigungschancen (und folglich der Arbeitslosigkeit) in der Bevölkerung auf. Desgleichen hat der durchschnittliche Erwachsene in Ländern mit relativ höherem Pro-Kopf-BIP mehr Bildungsjahre durchlaufen, doch schneidet der durchschnittliche 15-jährige Schüler nicht zwangsläufig besser ab. Die Korrelation zwischen Veränderungen dieser Autonomie-Indikatoren und des Pro-Kopf-BIP ist nur schwach.

- Soziale Gerechtigkeit: Sie spiegelt die Verteilung der Haushaltseinkommen sowie den Grad der Chancengleichheit für den Einzelnen wider. Sie kann anhand der Einkommensungleichheit, der relativen Armutsquoten, der Kinderarmut und dem Lohngefälle zwischen Männern und Frauen gemessen werden. Es besteht eine gewisse Korrelation zwischen höheren Niveaus des Pro-Kopf-BIP und einer vergleichsweise geringeren Ungleichheit der Einkommensverteilung. Zudem weisen OECD-Länder mit relativ niedrigerem Pro-Kopf-BIP in der Regel auch eine höhere relative Armut und Kinderarmut auf, was aber nicht zwangsläufig mit einem niedrigeren Lohngefälle zwischen Männern und Frauen einhergeht. Erhöhungen des Pro-Kopf-BIP sind assoziiert mit einem Rückgang der Einkommensungleichheit und des Lohngefälles zwischen den Geschlechtern, hingegen besteht eine nur sehr schwache bzw. gar keine Korrelation mit Veränderungen der Kinderarmut bzw. der relativen Armut.

- Gesundheitszustand: Dieser Indikator erfasst nicht nur Krankheiten und deren Behandlung, sondern auch andere soziale Faktoren, die Mortalität und Morbidität beeinflussen können. Die hier verwendeten vier Schlüsselindikatoren für den Gesundheitszustand sind die Lebenserwartung bei Geburt, die "gesunde" Lebenserwartung bei Geburt (d.h. die Lebensspanne, die nicht durch Behinderungen/ Krankheiten eingeschränkt ist), die Säuglingssterblichkeitsraten und die potenzielle Verkürzung der Lebenserwartung durch Unfälle oder vermeidbare Krankheiten. Diese Indikatoren sind stark mit dem Pro-Kopf-BIP korreliert, was bedeutet, dass der Gesundheitszustand der Bevölkerung in OECD-Ländern mit höherem Einkommen durchschnittlich besser ist. Gleichwohl kann es signifikante Differenzen zwischen den Ländern geben - so unterscheiden sich z.B. die Säuglingssterblichkeitsraten zwischen Ländern mit vergleichbarem Pro-Kopf-BIP um einen Faktor von bis zu rd. 2. Zwar sind Veränderungen des Pro-Kopf-BIP positiv mit Veränderungen des Gesundheitszustands korreliert, doch ist diese Korrelation nur schwach ausgeprägt und statistisch nicht signifikant.

- Das Gefühl der Zugehörigkeit zu einer größeren Gemeinschaft und die Zufriedenheit, die sich von der Teilhabe am allgemeinen Leben der Gesellschaft ableitet, sind wichtige Elemente des individuellen Wohlbefindens. Der soziale Zusammenhalt wird aber nicht nur 
mit Hilfe von positiven Indikatoren gemessen, wie dem Anteil der Menschen, die ehrenamtlich in örtlichen Einrichtungen tätig sind, sondern auch anhand negativer Indikatoren, wie z.B. des Niveaus der Kriminalität und der Viktimisierung sowie der Suizidraten. Zwar sind ehrenamtliche Tätigkeiten in Ländern mit höherem Pro-Kopf-BIP weiter verbreitet, doch besteht keine signifikante Korrelation mit den negativen Indikatoren, auch wenn eine Erhöhung des Pro-Kopf-BIP effektiv mit einem Rückgang der Zahl der Opfer von Gewalttaten Hand in Hand zu gehen scheint.

Alles in allem liefern die Sozialindikatoren Informationen über bestimmte Aspekte des Wohlergehens, die allem Anschein nach über die diesbezüglichen Aussagen des BIP hinausgehen ${ }^{4}$. Die wichtigste Schwachstelle der Sozialindikatoren besteht jedoch darin, dass sie wegen des mangelnden Konsenses darüber, wie sie zu aggregieren sind, keine globale Darstellung des Wohlergehens erlauben. Eine einfache synthetische Messgröße lässt sich jedoch konstruieren, indem die 16 weiter oben beschriebenen Indikatoren normalisiert und dann in einem Gesamtindex zusammengefasst werden, mit dem Vergleiche zwischen den verschiedenen Ländern möglich sind ${ }^{5}$. Dieser Index muss sodann daraufhin getestet werden, inwieweit er gegenüber unterschiedlichen Gewichtungen bei der Aggregierung der verschiedenen Grundindikatoren robust ist. Die hierbei angewandten Methoden sind beschrieben bei Boarini et al. (2006).

Abbildung 2.2 zeigt den Medianwert und das Konfidenzintervall für einen Gesamtindex, der mit Hilfe der sechzehn jeweils unterschiedlich gewichteten Sozialindikatoren konstruiert wurde. Der so gewonnene Gesamtindex der Sozialindikatoren weicht in etwas

\section{Abbildung 2.2. Medianwert und Konfidenzintervall eines Mehrkomponentenindex auf der Basis ausgewählter Sozialindikatoren in den OECD-Ländern und Pro-Kopf-BIP}

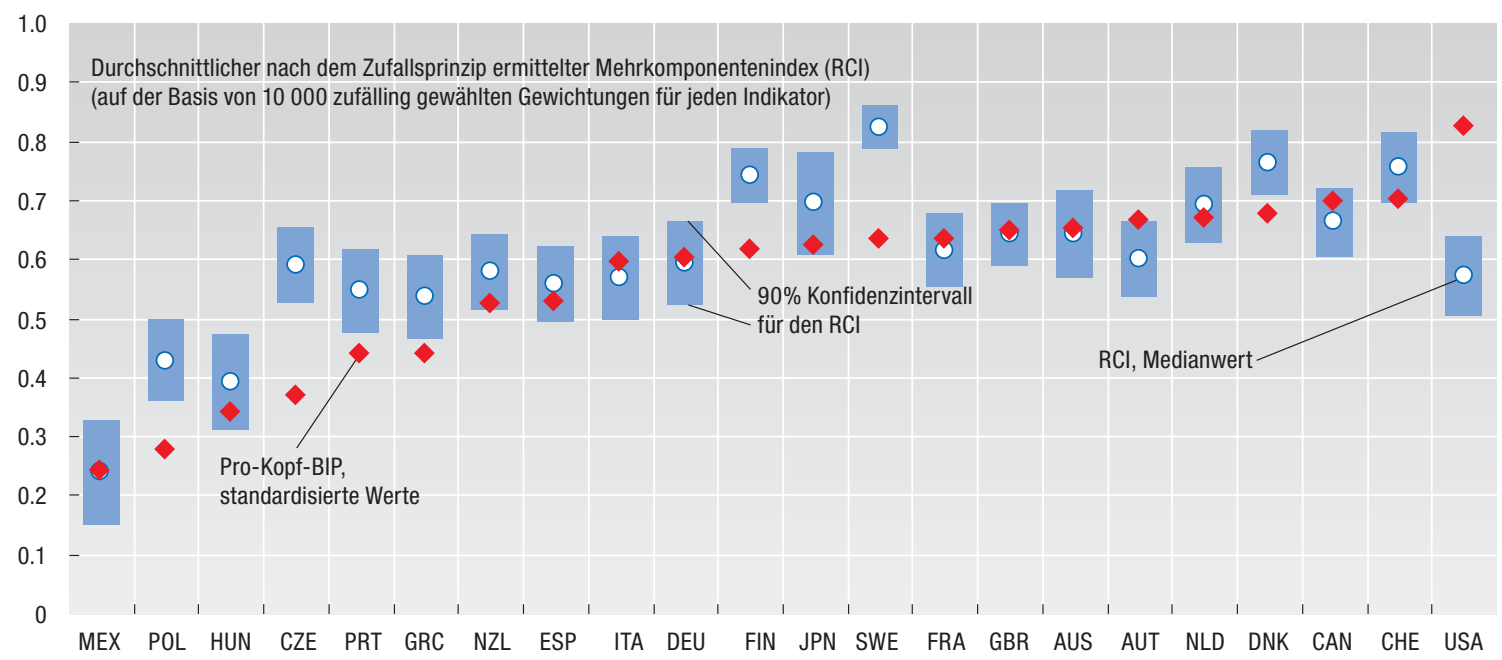

Anmerkung: Der zusammengesetzte Index basiert auf den Werten der 16 in Abbildung 2.1 dargestellten Sozialindikatoren. Die Analyse beschränkt sich auf diejenigen OECD-Länder, für die mindestens 13 der 16 Indikatoren verfügbar waren. Um Vergleiche zwischen dem zusammengesetzten Index der Sozialindikatoren und dem Pro-Kopf-BIP zu ermöglichen, wurden die Werte des Pro-Kopf-BIP auf einer Skala angeordnet, die durch den minimalen und den maximalen Medianwert des zusammengesetzten Index begrenzt wird. Der Medianwert und das Konfidenzintervall von $90 \%$ basieren auf 10000 Tests, bei denen jedem der Hauptindikatoren eine Gewichtung nach dem Zufallsprinzip zugeordnet wurde, wobei die Werte sodann mit dem (standardisierten) Pro-Kopf-BIP von 2001 verglichen wurden. Luxemburg wurde von der Analyse ausgeschlossen, um jede etwaige Verzerrung auf Grund seines “anormal” hohen Pro-Kopf-BIP zu vermeiden.

Quelle: Berechnungen auf der Basis von Daten aus verschiedenen Ausgaben von Gesellschaft auf einen Blick - OECDSozialindikatoren. 
mehr als der Hälfte der Länder signifikant von den mit Hilfe des Pro-Kopf-BIP gefundenen Ergebnissen $a b^{6}$. Bei Anwendung anderer methodischer Ansätze zur Konstruktion der Gesamtindizes erhält man ähnliche Resultate. Im Großen und Ganzen lassen die verschiedenen in einzelnen OECD-Ländern entwickelten Gesamtindizes ein übereinstimmendes Muster erkennen: Seit Beginn der siebziger Jahre ist das Wohlergehen weit weniger gestiegen als das Pro-Kopf-BIP und ist offenbar in den letzten Jahren sogar gesunken (Sharpe, 1999).

\section{Monetäre Messgrößen der wirtschaftlichen Ressourcen}

Der zweite Ansatz zur Messung des Wohlergehens besteht darin, von einer der verschiedenen Methoden des Systems der Volkswirtschaftlichen Gesamtrechnungen zur Berechnung des Realeinkommens auszugehen. Dieses Konzept beruht zwar auf solideren Grundlagen, das Problem der Vergleichbarkeit zwischen den Ländern besteht jedoch fort. Auch wird die Wirkung nichtmonetärer Faktoren auf das Wohlergehen außer Acht gelassen.

Wie schon erwähnt, ist das Pro-Kopf-BIP die am häufigsten herangezogene monetäre Messgröße zur Evaluierung des Gesamtwerts der wirtschaftlichen Ressourcen, die das Wohlergehen beeinflussen. Das BIP entspricht dem Wert der von einem Land in einem gegebenen Zeitraum erzeugten Güter und Dienstleistungen, was in der Praxis bedeutet, dass die Produktion derjenigen Wirtschaftszweige gemessen wird, die unter die Volkswirtschaftlichen Gesamtrechnungen fallen. Die erzeugten Güter und Dienstleistungen werden im Allgemeinen zu Marktpreisen unter der Annahme bewertet, dass diese Preise (für den Einzelnen wie für die Gesellschaft) exakt den Wert der für die Produktion verwendeten Ressourcen widerspiegeln, die ja auch anderweitigen Verwendungszwecken zugeführt werden könnten. Einige Aktivitäten, die in die Berechnung des BIP einbezogen werden, sind jedoch besonders schwer zu evaluieren. Öffentliche Dienstleistungen z.B. werden den direkten Nutzern häufig gebührenfrei oder zu subventionierten Preisen zur Verfügung gestellt, sodass die diesbezügliche Produktion nicht zu Marktpreisen evaluiert werden kann. In der Vergangenheit wurde für derartige Schätzungen der Wert der Inputs herangezogen, was darauf hinausläuft, die vom staatlichen Sektor erbrachten Dienstleistungen mit deren Produktionskosten gleichzusetzen. Einige OECD-Länder, wie z.B. das Vereinigte Königreich, haben in jüngster Zeit eine Umstellung vollzogen, indem sie damit begonnen haben, die Veränderungen der staatlichen Produktion auf der Basis direkter Output-Messungen zu erfassen. So umstritten diese Berichtigungen auch sind, haben sie doch signifikante Konsequenzen: Atkinson (2005) schätzt, dass die unterschiedlichen methodologischen Ansätze zur Berechnung der staatlichen Produktion nahezu die Hälfte der Differenz zwischen den BIPWachstumsraten für das Vereinigte Königreich und die Vereinigten Staaten im Zeitraum 1995-2003 erklären.

Bei der Evaluierung der erbrachten Güter und Dienstleistungen zu Marktpreisen wird unterstellt, dass die Preise den marginalen Beitrag der verschiedenen konsumierten Güter zum Nutzen des Einzelnen repräsentieren. Bei diesem Konzept stellt das Pro-Kopf-BIP jedoch lediglich eine indirekte Messgröße für das Wohlergehen dar, d.h. eine Reihe ebenfalls wichtiger Faktoren bleibt unberücksichtigt:

- Das BIP lässt eine Reihe nicht marktbestimmter Aktivitäten außer Acht, die das Wohlergehen ebenfalls beeinflussen; der Grund hierfür sind praktische Probleme bei 
deren Messung, weil der Marktwert nur schwer zu quantifizieren ist. Dazu zählen nicht nur illegale Tätigkeiten oder häusliche Tätigkeiten wie Hausarbeit und Heimwerken, sondern auch Freizeitaktivitäten, die von nicht zu leugnender Bedeutung für die Gesellschaft und wesentlich für das Wohlbefinden des Einzelnen sind.

- Bei den klassischen BIP-Messungen bleiben Veränderungen der Vermögenswerte unberücksichtigt, obwohl diese natürlich auf jeden Fall einen Einfluss darauf haben, wie viel eine Person zu einem gegebenen Zeitpunkt für Konsumzwecke ausgeben kann, ohne dadurch ihre künftige finanzielle Situation zu gefährden. Oder anders gesagt, das BIP misst eher das, was die Gesellschaft produziert, als das, was sie potenziell konsumiert.

- Das BIP trägt nicht den Externalitäten Rechnung, wie z.B. der Umweltverschmutzung, der Verschlechterung der Umweltbedingungen oder dem Raubbau an nicht erneuerbaren Ressourcen. Dadurch wird das Bild verzerrt, das die Marktpreise vom marginalen Beitrag zeichnen, den bestimmte Faktoren effektiv zum Wohlergehen der Gesellschaft - namentlich der künftigen Generationen - leisten.

- Das BIP trägt nicht den Unterschieden der Einkommensverteilung innerhalb der einzelnen Länder Rechnung. Für den größten Teil der Bevölkerung hat eine enorme Zunahme des Nationaleinkommens, die ausschließlich einer kleinen Zahl sehr reicher Familien zugute kommt, nicht die gleiche Steigerung des allgemeinen Wohlergehens zur Folge, wie dies bei einer gerechteren Verteilung dieses Einkommens der Fall wäre.

Aus diesen und anderen Gründen wurden gewisse Berichtigungen der auf den Volkswirtschaftlichen Gesamtrechnungen beruhenden Messungen vorgenommen, um alternative monetäre Messgrößen für das Wohlergehen zu entwickeln.

\subsection{Bruttonationaleinkommen: Berichtigung um Nettotransfers aus dem Ausland}

Das BIP trägt lediglich dem Produktionsprozess Rechnung, der sich innerhalb der Grenzen eines Landes vollzieht, lässt jedoch unberücksichtigt, dass ein Teil des durch diese Aktivitäten generierten Einkommens an Gebietsfremde gezahlt wird, während Gebietsansässige wiederum Einkommen aus Produktionsprozessen in anderen Ländern beziehen. Die Kaufkraft Gebietsansässiger kann in Bezug auf ausländische Güter auch infolge von Veränderungen der Terms of Trade - d.h. der Relation zwischen Import- und Exportpreisen - zu- oder abnehmen. Bei Einbeziehung des "Nettoeinkommens aus dem Ausland" in das BIP erhält man das Bruttonationaleinkommen (BNE), ein Konzept, das für die Messung des Wohlergehens der Gebietsansässigen eines Landes relevanter ist.

Um Ländervergleiche dieser Daten zu ermöglichen, müssen die in Landeswährung ausgedrückten Produktionsdaten mit Hilfe von Kaufkraftparitäten-Wechselkursen (KKP) in eine gemeinsame Währung umgewandelt werden. In den meisten OECD-Ländern ist die Differenz zwischen Pro-Kopf-BIP und Pro-Kopf-BNE gering, da die Zuflüsse an Bruttoeinkommen aus dem Ausland in der Regel ungefähr den Bruttoabflüssen entsprechen - allerdings mit einigen bemerkenswerten Ausnahmen wie z.B. Irland und Schweiz (Abb. 2.3). In den vergangenen zehn Jahren waren die Veränderungen des ProKopf-BIP und des Pro-Kopf-BNE weitgehend analog; eine Ausnahme bilden Irland und Südkorea, d.h. Länder, die in großem Maßstab IKT-Produkte herstellen und daher eine relativ drastische Verschlechterung ihrer Terms of Trade hinnehmen mussten. 


\section{Abbildung 2.3. Bruttoinlandsprodukt sowie Pro-Kopf-Brutto- und -Nettonationaleinkommen in den OECD-Ländern}

Zu laufenden Preisen und KKP in US-\$

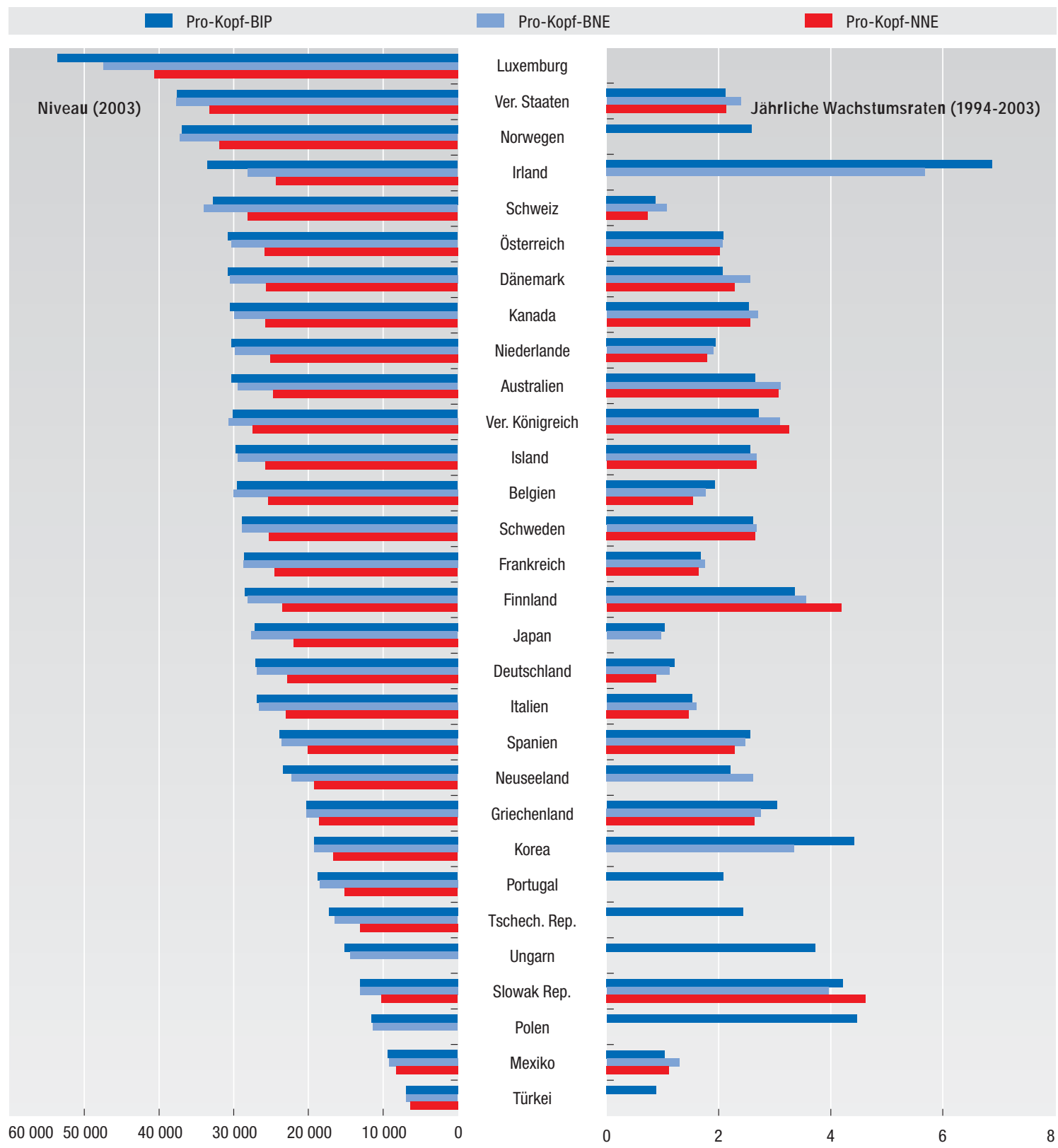

Anmerkung: Die Länder sind von oben nach unten in abnehmender Rangfolge ihres Pro-Kopf-BIP angeordnet.

Quelle: OECD Annual National Accounts.

S tat Link 2 http://dx.doi.org/10.1787/025143474403

\subsection{Nettonationaleinkommen: Berichtigung um Abschreibungen}

Das BIP trägt nicht dem Kapitalverschleiß während des Produktionsprozesses Rechnung und überschätzt folglich den Wert der Produktion, der effektiv zum Wohlergehen beiträgt, ohne die künftige Produktion zu beeinträchtigen. Zur Berichtigung werden die Kapitalabschreibungen geschätzt und sodann vom BIP subtrahiert, um das 
Nettoinlandsprodukt (NIP) zu erhalten. Dieser Parameter entspricht dem maximalen Umfang der Produktion, der dem Konsum zugeführt werden kann, ohne dass dadurch die Produktionskapazität eines Landes beeinträchtigt wird. Wenn auch alle Länder Schätzungen über die Abschreibungen erstellen, sind doch die Berechnungsmethoden unterschiedlich, was die internationale Vergleichbarkeit der NIP-Messungen einschränkt.

Trotzdem variiert die Differenz zwischen Pro-Kopf-BIP und Pro-Kopf-NIP von Jahr zu Jahr kaum, und zwar ebenso wenig wie die Rangfolge der Länder auf der Basis dieser beiden Kriterien. Das Pro-Kopf-NIP beträgt in den OECD-Ländern durchschnittlich $85 \%$ des Pro-Kopf-BIP. In den letzten zehn Jahren ist es jedoch etwas langsamer gewachsen als das Pro-Kopf-BIP, was darauf zurückzuführen ist, dass die Abschreibungen auf Grund der zunehmenden Investitionen in neue Technologien mit kürzerer Nutzungsdauer stärker gestiegen sind als das BIP.

Ähnlich wie das BIP kann auch das NIP um den Effekt des "Nettoeinkommens aus dem Ausland" berichtigt werden, um das Nettonationaleinkommen (NNE) zu erhalten. Berücksichtigt man die Probleme bei der Berechnung der Abschreibungen, so vermittelt dieser Parameter im Prinzip ein genaueres Bild der zur Gewährleistung des Wohlergehens aller Bürger des jeweiligen Landes effektiv verfügbaren wirtschaftlichen Ressourcen und bestätigt, dass das Pro-Kopf-BIP diese tendenziell effektiv zu hoch ansetzt. Gleichwohl ist die Rangfolge der Länder auf der Basis des Pro-Kopf-NNE insgesamt ähnlich wie auf der Basis des Pro-Kopf-BIP, wobei die Differenz für einige wenige Länder allerdings signifikant ist (Abb. 2.3). Auch die Wachstumsraten sind für die beiden Messgrößen im Großen und Ganzen sehr ähnlich.

\subsection{Messung der wirtschaftlichen Ressourcen der privaten Haushalte}

Die vorstehend beschriebenen Gesamtgrößen ermöglichen es lediglich, die Produktion bzw. das Einkommen der gesamten Volkswirtschaft zu messen. Der Begriff des Wohlergehens bezieht sich jedoch hauptsächlich auf den Einzelnen bzw. auf die Haushalte. Die diesen zur Verfügung stehenden wirtschaftlichen Ressourcen, einschließlich der ihnen vom Staat und von gemeinnützigen Einrichtungen gebührenfrei bereitgestellten Gütern und Dienstleistungen, vermitteln ein genaueres Bild ihres wirtschaftlichen Wohlergehens. Das lässt sich ausgehend von der Volkswirtschaftlichen Gesamtrechnung auf dreierlei Art und Weise berechnen: anhand des verfügbaren Pro-Kopf-Einkommens, des Pro-KopfEndverbrauchs und des "effektiven" Pro-Kopf-Verbrauchs der Haushalte, der eine Schätzung der vom Staat und von gemeinnützigen Einrichtungen erbrachten Dienstleistungen einschließt? .

Alle drei Messgrößen, und insbesondere der Endverbrauch, fallen natürlich signifikant niedriger aus als das Pro-Kopf-BIP. Gleichwohl korrelieren alle stark mit dem Pro-Kopf-BIP, auch wenn die Diskrepanz zwischen dem verfügbaren Einkommen und dem Pro-Kopf-BIP von $20 \%$ in der Türkei bis zu $57 \%$ in Dänemark reicht. In den letzten zehn Jahren sind die Einkommen der privaten Haushalte und der effektive Konsum in den meisten Ländern jedoch weniger rasch gestiegen als das Pro-Kopf-BIP (Abb. 2.4) - wobei die Differenz in einigen Ländern nicht weniger als 1 Prozentpunkt ausmacht -, was sich aus Einkommensumschichtungen zwischen privaten Haushalten, Unternehmen und öffentlichem Sektor erklärt. 


\section{Abbildung 2.4. Verfügbares Realeinkommen der privaten Haushalte, reale Endverbraucherausgaben und reales Pro-Kopf-BIP, durchschnittliche jährliche Wachstumsrate, 1994-2003}

In Prozent
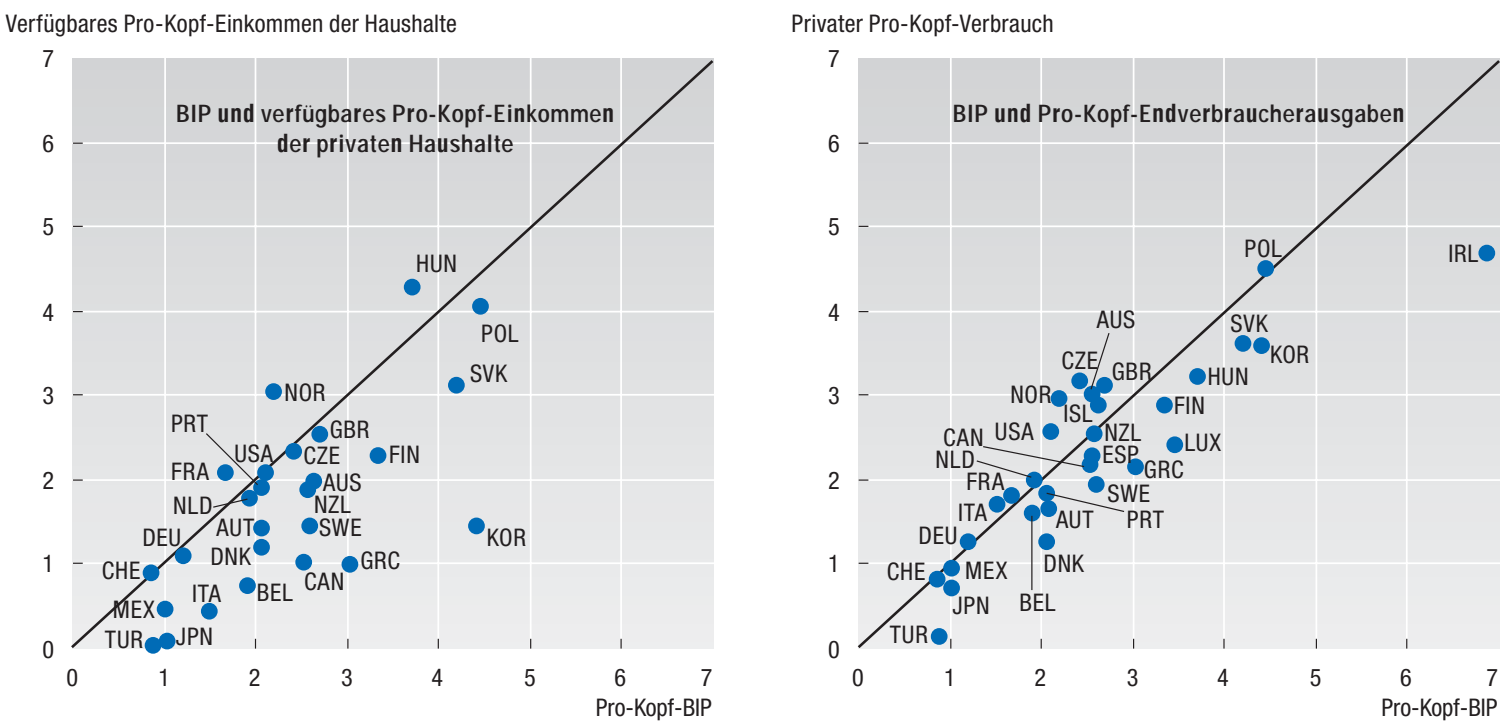

Source: OECD Annual National Accounts und OECD (2005), OECD-Wirtschaftsausblick 76, Paris.

StatLink 2 http://dx.doi.org/10.1787/602121645683

\subsection{Zusammenfassende Bemerkungen zu den monetären Indikatoren}

Bleibt man im Rahmen der Volkswirtschaftlichen Gesamtrechnung, so ist alles in allem und ungeachtet der unterschiedlichen Berichtigungen ein recht hoher Grad an Konvergenz beim Niveau und in geringerem Maße auch bei den Wachstumsraten der verschiedenen Indikatoren für die in einem Land insgesamt verfügbaren wirtschaftlichen Ressourcen festzustellen. Je stärker sich die Messungen aber auf die den privaten Haushalten zur Gewährleistung ihres Wohlergehens effektiv zur Verfügung stehenden wirtschaftlichen Ressourcen konzentrieren, desto geringer wird die Konvergenz mit den Messgrößen für die Ressourcen der Volkswirtschaft insgesamt.

Die vorstehend erörterten Indikatoren der wirtschaftlichen Ressourcen messen einen Schlüsselfaktor des Wohlergehens des Einzelnen wie auch der Gesellschaft insgesamt. So wichtig die wirtschaftlichen Ressourcen aber auch sein mögen, sind sie doch für sich alleine genommen nicht ausschlaggebend für das Wohlbefinden des Einzelnen - denn wie schon das Sprichwort sagt, "Geld allein macht nicht glücklich". Das scheint sich in dem Maße zu bestätigen, wie eine Gesellschaft den Punkt überschreitet, ab dem sie die Grundbedürfnisse der Bevölkerung in Bezug auf Ernährung, Unterkunft und Bekleidung zu befriedigen imstande ist. Die Ökonomen haben sel bst diese Grenze erkannt und haben sich um die Entwicklung verschiedener anderer Mechanismen zur Berücksichtigung nichtmarktbezogener Faktoren bemüht ${ }^{8}$.

\section{Weitere Berichtigungen der Messgrößen auf der Basis der Volkswirtschaftlichen Gesamtrechnung}

Diese Messgrößen der wirtschaftlichen Ressourcen können zusätzlich berichtigt werden, indem verschiedene nichtmonetäre Faktoren mit einem monetären Wert 
versehen werden, um auf diese Weise eine bessere Hilfsvariable für das Wohlergehen des Einzelnen und der Gesellschaft zu erhalten. Die Hauptschwierigkeit besteht darin, den Wert verschiedener Aktivitäten des Nichtmarktbereichs, wie z.B. den Geldwert von Freizeit und nicht erfassten wirtschaftlichen Tätigkeiten wie Hausarbeit, zu beziffern. Die verschiedenen Schätzungen bewerten diese Aktivitäten zumeist auf der Basis der Wiederbeschaffungs- oder Opportunitätskosten. Nachstehend wird eine Reihe von Ergebnissen dargestellt, die eine Vorstellung von der Wirkung einiger dieser nichtmarktbestimmten Faktoren geben.

\subsection{Wohlergehen und Freizeit}

Die vom BIP abgeleiteten Messgrößen sind ein unvollständiger Indikator für das Wohlergehen, weil die Freizeit unberücksichtigt bleibt. Freizeit hat aber zweifellos große Bedeutung für das Wohlbefinden der meisten Menschen. So gesehen handelt es sich um ein "Gut" mit einem gewissen Grenznutzen. Wie lässt sich aber dieses Gut quantifizieren? Und wie kann ihm ein monetärer Wert beigemessen werden? Wir sind sicherlich noch weit davon entfernt, zufriedenstellende Antworten auf diese Fragen zu haben. Die erheblichen Diskrepanzen zwischen der effektiven entgeltlichen Jahresarbeitszeit je Erwerbstätigen in den einzelnen Ländern lassen jedoch darauf schließen, dass es auch große quantitative Unterschiede in Bezug auf die Freizeit gibt. Ein Teil der Differenz zwischen dem Pro-KopfBIP der Vereinigten Staaten und der meisten anderen OECD-Länder ist darauf zurückzuführen, dass die Erwerbstätigen in den USA jährlich insgesamt mehr Arbeitsstunden leisten. Inwieweit ist dies durch kulturelle Unterschiede und/oder Präferenzen bedingt oder aber durch die Effekte von Politik und Institutionen? Auch wenn sich diese Fragen nicht präzise beantworten lassen, liegt doch auf der Hand, dass eine realistische Evaluierung des Wohlergehens nur möglich ist, wenn der Freizeit der Arbeitnehmer ein gewisser monetärer Wert beigemessen wird ${ }^{9}$.

Das hier gewählte Konzept besteht darin, das Pro-Kopf-BIP um eine quantitative Schätzung der jährlichen Freizeit je Arbeitnehmer zu erweitern, wobei der monetäre Wert mit Hilfe dreier verschiedener Messgrößen ermittelt wird, nämlich BIP pro Arbeitsstunde, Stundenlohn je Arbeitnehmer und $50 \%$ dieses Stundenlohns (um der Möglichkeit Rechnung zu tragen, dass die niedrigere Zahl der Arbeitsstunden in einigen Ländern auf die Wirkung von Steuern und Abgaben sowie andere Maßnahmen zurückzuführen ist). Ermittelt man mit Hilfe dieser drei Ansätze das "freizeitberichtigte" Pro-Kopf-BIP und das diesbezügliche Gefälle gegenüber den Vereinigten Staaten, so zeigt sich, dass jede positive Bewertung der Freizeit den Abstand gegenüber den auf der Basis des Pro-Kopf-BIP durchgeführten Berechnungen verringert (je höher der Wert der Freizeit angesetzt wird, desto geringer ist der Abstand). Das durchschnittliche jährliche Wachstum des "freizeitberichtigten" Pro-Kopf-BIP ist vor allem in einigen europäischen Ländern tendenziell höher als das des nicht bereinigten BIP (Abb. 2.5).

\subsection{Wohlergehen und Haushaltsgröße}

Das Pro-Kopf-Haushaltseinkommen wird in den Volkswirtschaftlichen Gesamtrechnungen geschätzt, indem die Summe der Einkommen sämtlicher Haushalte durch die Zahl der Gebietsansässigen dividiert wird. Bei diesem Ansatz bleiben etwaige Veränderungen der Haushaltsgröße unberücksichtigt. In der Tat verfügen die Haushalte je nach Größe über unterschiedliche Kapazitäten für die Zusammenlegung finanzieller Mittel und benötigen daher nicht immer das gleiche Einkommen, um ihren Mitgliedern das 


\section{Abbildung 2.5. Durchschnittliche jährliche Wachstumsrate des um die Freizeit der Arbeitnehmer berichtigten BIP, 1970-2003}

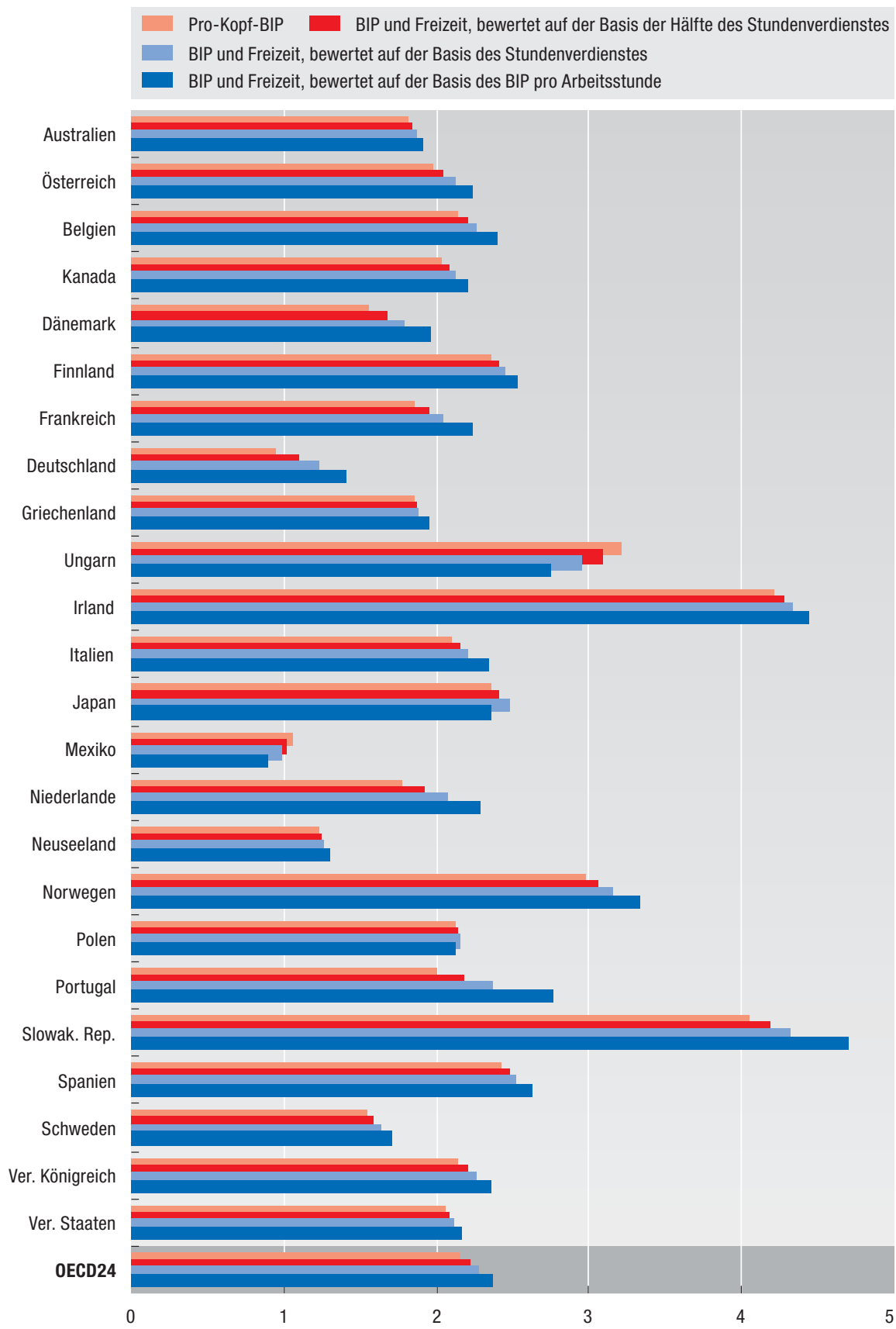

Anmerkung: Der Umfang der Freizeit seines Arbeitnehmers wird geschätzt, indem von der Zeit, über die jeder Arbeitnehmer verfügt, die (für alle identische) geschätzte Zeit, die der persönlichen Pflege und unbezahlten Aktivitäten gewidmet ist, sowie die für das jeweilige Land geschätzten jährlichen Arbeitsstunden je Arbeitnehmer abgezogen werden. Der Wert der Freizeit wird zu drei verschiedenen Preisen berechnet: Stundenverdienst der abhängig Beschäftigten, 50\% dieses Stundenverdienstes und BIP je Arbeitsstunde. Der Beobachtungszeitraum reicht für die meisten Länder von 1970 bis 2003, ist für einige jedoch kürzer (Deutschland, Griechenland, Korea, Luxemburg, Mexiko, Neuseeland, Österreich, Portugal, Slowakische Republik, Tschechische Republik und Ungarn). Wegen näherer Einzelheiten vgl. Boarini et al. (2006).

Quelle: OECD Productivity database und Annual National Accounts. 
gleiche Niveau des Wohlergehens zu sichern. So benötigt z.B. ein Haushalt, der aus einem Paar mit zwei Kindern besteht, nicht zwangsläufig das doppelte Einkommen wie ein kinderloses Paar, um das gleiche Niveau des Wohlergehens zu erreichen. Eine Möglichkeit, diesen Faktor zu berücksichtigen, besteht darin, auf die Erhebungsdaten des Haushaltseinkommens eine gemeinsame "Äquivalenzskala" anzuwenden, um das so genannte "verfügbare Äquivalenzeinkommen" jedes Einzelnen zu berechnen.

Äquivalenzskalen sind Rechenhilfen, über deren "wirklichen" Wert es keinen vollständigen empirischen Konsens gibt; sie können zudem von Land zu Land wie auch im Zeitverlauf variieren. Wenn das Niveau des verfügbaren Äquivalenzeinkommens daher auch keinen besonders großen Informationswert besitzt - Schätzungen zeigen erwartungsgemäß, dass das verfügbare Äquivalenzeinkommen der privaten Haushalte höher ist als das Nichtäquivalenzeinkommen, dem ja die Annahme zu Grunde liegt, dass jede Person allein lebt und dass diese Differenz in Ländern mit umfangreicherer durchschnittlicher Haushaltsgröße ausgeprägter ist -, geht aus den Veränderungen des verfügbaren Äquivalenzeinkommens im Zeitverlauf doch hervor, dass der allgemeine Trend hin zu kleineren Familienverbänden die Skalenerträge und das Wohlergehen in allen Ländern verringert hat, zuweilen sogar in beträchtlichem Umfang (wie z.B. in Italien und Mexiko, Abb. 2.6).

\subsection{Ungleiche Verteilung der wirtschaftlichen Ressourcen}

In keinem der OECD-Länder ist das Einkommen gleichmäßig verteilt, und die OECDweiten Trends lassen seit Mitte der achtziger Jahre, namentlich in einer kleinen Zahl von Ländern, eine Zunahme der Ungleichheit erkennen (Förster und Mira d'Ercole, 2005). Die klassischen Methoden zur Messung des Pro-Kopf-BIP geben jeder Einkommenseinheit dassel be Gewicht, unabhängig davon, wie gleichmäßig das Einkommen verteilt ist. Viele Verfechter von Theorien der sozialen Gerechtigkeit würden jedoch dafür plädieren, dem Einkommen, das den ärmsten Bevölkerungsschichten zugute kommt, eine höhere Gewichtung beizumessen, vor allem in den Gesellschaften, in denen die Ungleichheit besonders stark ausgeprägt ist. Nimmt man z.B. den Fall, dass das Einkommen des reichsten Bevölkerungsdezils in demselben Umfang zunimmt, wie das Einkommen des ärmsten Bevölkerungsdezils abnimmt, dann bleibt das Pro-Kopf-Einkommen zwar unverändert, doch wären sich die meisten Beobachter darüber einig, dass sich das allgemeine Wohlergehen der Gesellschaft verschlechtert hat.

Um dem Problem der ungleichen Einkommensverteilung Rechnung zu tragen, können das BIP und das Haushaltseinkommen berichtigt werden, indem das Durchschnittseinkommen in jedem Dezil der Einkommensverteilung mit einem Koeffizienten gewichtet wird, der den Grad der sozialen Aversion gegenüber Ungleichheit repräsentiert (Kolm, 1969). Abbildung 2.7 zeigt die Ergebnisse von Berechnungen, die mit drei verschiedenen Gewichtungen angestellt wurden. Höhere Koeffizienten stehen für einen höheren Grad der Aversion und ziehen folglich eine geringere Gewichtung höherer Einkommen nach sich. In Ländern, in denen das Einkommenswachstum groß enteils den besser gestellten Bevölkerungsschichten zugute kommt, wird sich die Anwendung eines höheren Koeffizienten in einer geringeren jährlichen Veränderung des Haushaltseinkommens niederschlagen (Vereinigtes Königreich, Tschechische Republik, Vereinigte Staaten), während in Ländern, wo die ärmeren Bevölkerungsschichten stärker vom Einkommenswachstum profitieren, eine tendenzielle Zunahme der jährlichen Veränderung die Folge ist (Mexiko, Spanien, Norwegen). Während ein niedriger oder sogar mittlerer Grad der Aversion gegenüber 


\section{Abbildung 2.6. Jährliche reale Veränderung des verfügbaren Pro-Kopf- Haushaltseinkommens und Berichtigung um Veränderungen der Haushaltsgröße, 1995 bis Anfang der 2000er Jahre}

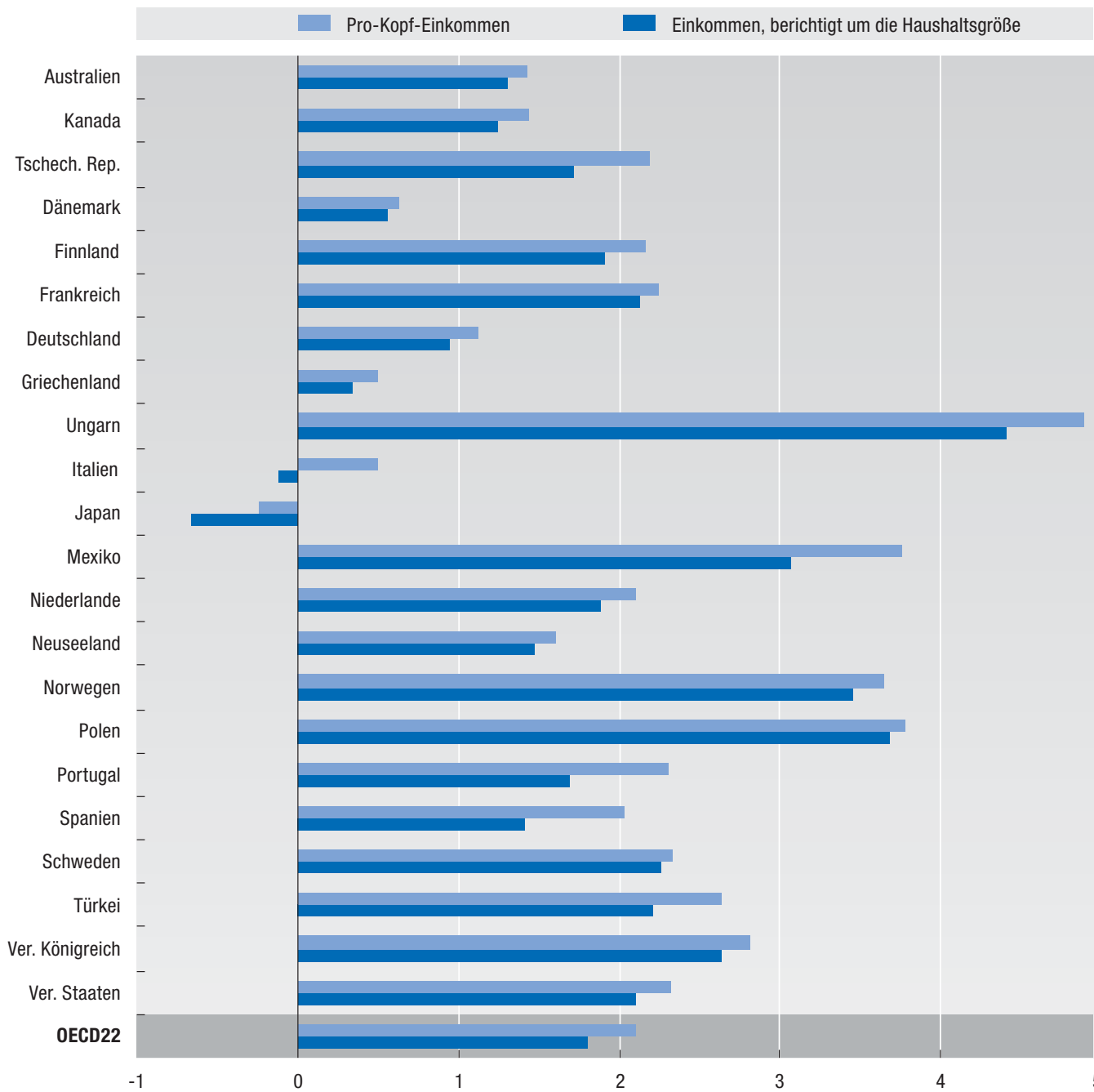

Anmerkung: Die Erhebungsdaten über das verfügbare Haushaltseinkommen beziehen sich nicht auf eine chronologische Folge von Jahren, sondern auf jeweils einzelne Jahre (Mitte der 80er, Mitte der 90er und Anfang der 2000er Jahre), die für die einzelnen Länder unterschiedlich sein können. Um Vergleiche zwischen den berücksichtigten Jahren zu ermöglichen, wurden diese Daten zwischen den vorliegenden Beobachtungen interpoliert und (wenn notwendig) bis 2002 extrapoliert. Die (aus diesen Erhebungen stammenden) Daten über die Durchschnittsgröße der privaten Haushalte wurden auf die "aggregierte" Messgröße der Volkswirtschaftlichen Gesamtrechnung für das verfügbare Einkommen der privaten Haushalte angewandt (um das Problem der Vergleichbarkeit wegen der unterschiedlichen, einerseits auf Erhebungen und andererseits auf der Volkswirtschaftlichen Gesamtrechnung basierenden Messgrößen des verfügbaren Einkommens der privaten Haushalte zu vermeiden).

Quelle: Berechnungen auf der Basis der OECD National Accounts und des OECD-Fragebogens über Einkommensverteilung und Armut.

StatLink 2 http://dx.doi.org/10.1787/534122818370

Ungleichheit die Rangfolge der Länder nicht wesentlich verändert, bewirkt ein höherer Aversionsgrad signifikante Veränderungen. Bei Anwendung eines Koeffizienten, der dem höchsten Aversionsgrad entspricht, sinkt auch die bereinigte Zuwachsrate des verfügbaren Einkommens im Zeitraum 1985-2002 auf 0,6\%, verglichen mit 1,4\% beim klassisch berechneten Einkommen, wobei der Rückgang in einigen Ländern sogar noch stärker ausfällt 


\section{Abbildung 2.7. Jährliche reale Veränderung des verfügbaren Einkommens der privaten Haushalte auf der Basis verschiedener Werte des Koeffizienten der Aversion gegenüber Ungleichheit}

Durchschnittliche jährliche Wachstumsrate, 1995-2002

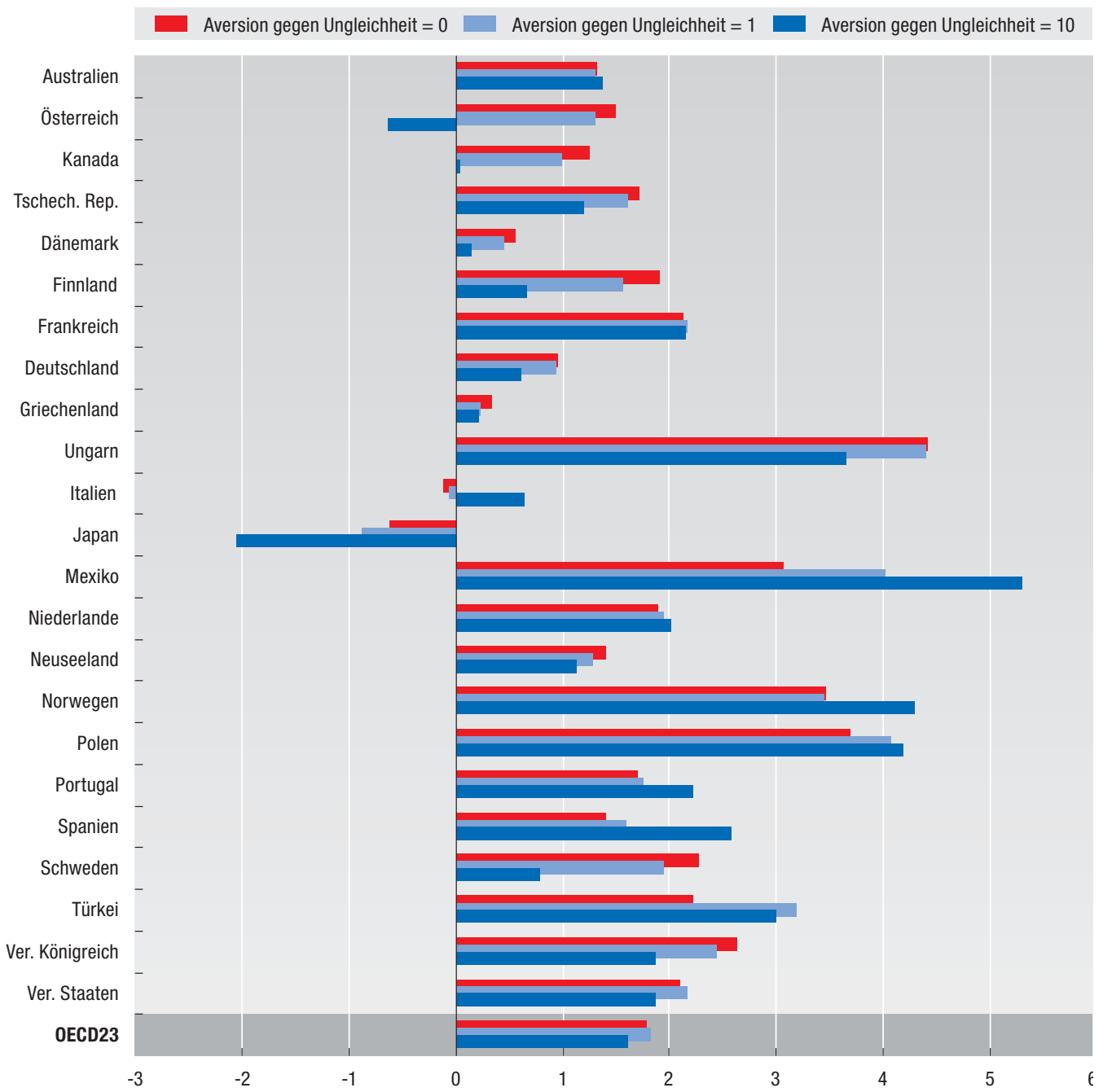

Anmerkung: Die nationalen Werte des "gleichmäßig verteilten" verfügbaren Einkommens der privaten Haushalte werden berechnet als Durchschnitt der Werte für jedes Dezil, unter Verwendung von drei Koeffizienten der Aversion gegenüber Ungleichheit, nämlich 0, 1 und 10. Das Niveau des verfügbaren Haushaltseinkommens wird je Dezil berechnet als Produkt aus dem um die Haushaltsgröße berichtigten, auf der Basis der Volkswirtschaftlichen Gesamtrechnungen geschätzten verfügbaren Haushaltseinkommen und dem nach Erhebungen geschätzten Verhältnis zwischen dem verfügbaren Einkommen des jeweiligen Dezils und dem arithmetischen Mittel. Die Daten für das verfügbare Einkommen der privaten Haushalte werden anhand der Kaufkraftparitäten (KKP) für private Verbraucherausgaben in eine gemeinsame Währung umgerechnet; die Daten für das Pro-Kopf-BIP basieren auf den KKP für das BIP.

Quelle: Berechnungen auf der Basis der OECD National Accounts und des OECD-Fragebogens über Einkommensverteilung und Armut.

S tat Link 2 http://dx.doi.org/10.1787/533254380580

(Abb. 2.7). Abschließend lässt sich sagen, dass der Grad der Ungleichheit der Einkommensverteilung einen signifikanten Einfluss auf die Evaluierung des Wohlergehens im Vergleich zu den vom klassischen Einkommen ausgehenden Messungen haben kann, dass jedoch die Stärke dieses Effekts in einer gegebenen Gesellschaft maßgeblich vom unterstellten Grad der Aversion gegenüber Ungleichheit abhängt. 


\subsection{Wohlergehen und Umwelt}

Das Wohlergehen wird nicht allein durch soziale und ökonomische, sondern auch durch umweltbezogene Faktoren bestimmt. In der Tat lag den Untersuchungen über erweiterte Messgrößen des Wohlergehens weitgehend die Besorgnis über die Verschlechterung der Umweltbedingungen zu Grunde. Das Konzept der nachhaltigen Entwicklung unterstreicht die Notwendigkeit, auch solchen Ressourcen und Aktiva Rechnung zu tragen, die nicht in die klassischen ökonomischen Gesamtrechnungen eingehen. Obgleich das Konzept der nachhaltigen Entwicklung unmittelbare Implikationen für die Messung des Einkommens hat - namentlich auf der Ebene der Ressourcen und der ökologischen Werte, die durch die wirtschaftliche Produktion beeinflusst, aber bei den Markttransaktionen nicht berücksichtigt werden -, gibt es bislang noch keine etablierten Mechanismen zur Einbeziehung dieser Belange in die Messung der wirtschaftlichen Ressourcen ${ }^{10}$. Analog zum Sozialbereich bestehen ferner komplexe Beziehungen zwischen Umweltqualität und wirtschaftlicher Entwicklung. Ein höheres BIP-Niveau hat in der Regel eine tendenziell stärkere Umweltbelastung zur Folge, steigert aber andererseits auch die Kapazitäten und Ressourcen zur Behandlung von Umweltproblemen.

\subsection{Zusammenfassung der notwendigen Bereinigungen monetärer Messgrößen}

Die verschiedenen vorstehend beschriebenen Konzepte zur Berücksichtigung eines Teils der Faktoren, die das Wohlergehen beeinflussen, bei den herkömmlichen Berechnungen aber nicht berücksichtigt werden, beruhen auf der volkswirtschaftlichen Lehre. Die unterschiedlichen Methoden zur Bewertung dieser Faktoren führen jedoch zwangsläufig zu unterschiedlichen Schlussfolgerungen. Darüber hinaus bleiben andere Faktoren, die ebenfalls von Bedeutung sind, wie z.B. die Arbeit im Haushalt, Ausgaben zur Behebung gewisser durch das Wirtschaftswachstum verursachter Schäden sowie Umweltfaktoren, nach wie vor unberücksichtigt. Diese Faktoren sind zwar schwer messbar, aber ihre Nichtbeachtung kann insofern zu falschen Folgerungen führen, als sie im Zeitverlauf und von Land zu Land variieren ${ }^{11}$. Das ist besonders dann der Fall, wenn diese Faktoren unmittelbar durch wirtschaftliche Tätigkeiten beeinflusst werden.

\section{Wohlbefinden und Glücksempfinden}

Eine dritte Vorgehensweise besteht darin, das Wohlbefinden nicht mit Hilfe objektiver Messgrößen zu ermitteln, sondern ganz einfach die Menschen selbst zu fragen, wie zufrieden sie mit ihrem Leben sind. Bei der subjektiven Messung des Wohlbefindens tauchen aber natürlich Schwierigkeiten methodologischer Art auf. Diese können auf unterschiedliche Konzepte, zeitlich befristete Faktoren oder auch sprachliche bzw. kulturelle Unterschiede zurückgehen. Untersuchungen haben gleichwohl ergeben, dass Menschen, die ihren eigenen Angaben zufolge relativ zufrieden mit ihrem Leben sind, auch von ihren Verwandten und Freunden als relativ glücklich eingeschätzt werden, im täglichen Umgang mit ihren Mitmenschen häufiger eine freundliche Miene zur Schau stellen, sich durch eine intensivere Aktivität im präfrontalen Cortex (dem mit positiven Befindlichkeiten assoziierten Teil des Gehirns) auszeichnen, sich eher an glückliche Ereignisse ihres Lebens erinnern und eine höhere Stressresistenz aufweisen (Layard, 2005). Es gibt mehrere globale Untersuchungen, wie den Weltwertebericht (World Values Survey), die von vergleichbaren Kriterien ausgehen und bei denen eine repräsentative Stichprobe der Bevölkerung befragt wird, wie zufrieden sie mit ihrem Leben sind. 
Vergleiche subjektiver Indikatoren der Lebenszufriedenheit und des nationalen Durchschnittseinkommens führen zu zwei Schlussfolgerungen:

- Im Ländervergleich sind die Einwohner von Ländern mit höherem Pro-Kopf-BIP nach eigenen Angaben zu einem gegebenen Zeitpunkt meist glücklicher; der Zugewinn an subjektivem Wohlbefinden geht jedoch tendenziell zurück, sobald das Pro-Kopf-BIP 10000 US-\$ überschreitet (Frey und Stutzer, 2002). Dieser sinkende Trend ist indessen weniger ausgeprägt, wenn lediglich die OECD-Länder betrachtet werden (Abb. 2.8, linker Teil) und variiert je nach der verwendeten Messgröße des Nationaleinkommens (d.h. Pro-Kopf-BIP oder Pro-Kopf-NNE).

\section{Abbildung 2.8. Vergleich des subjektiven Wohlbefindens und des Pro-Kopf-BIP zwischen und in den OECD-Ländern}

Grad der Lebenszufriedenheit in den einzelnen OECD-Ländern

Lebenszufriedenheit (Durchschnittswerte)

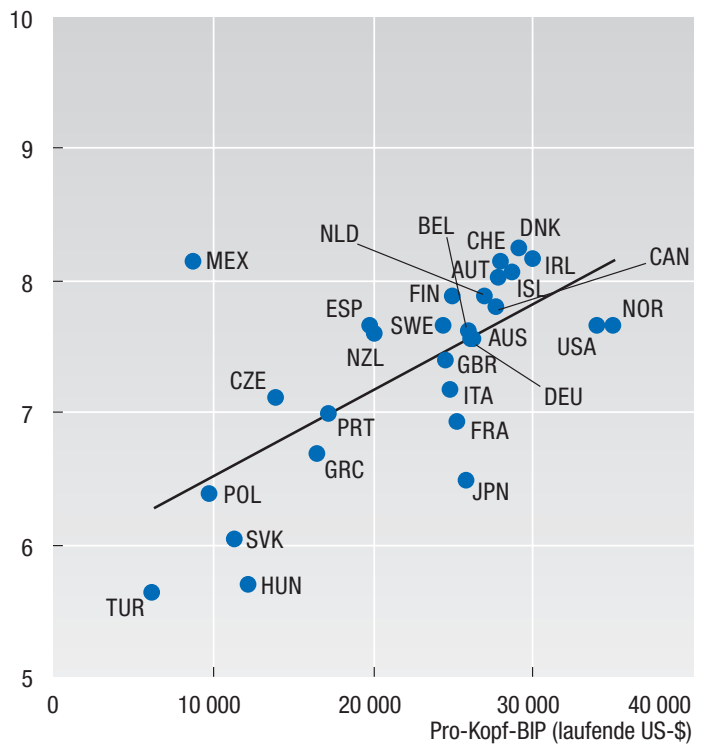

Trends in ausgewählten OECD-Ländern²

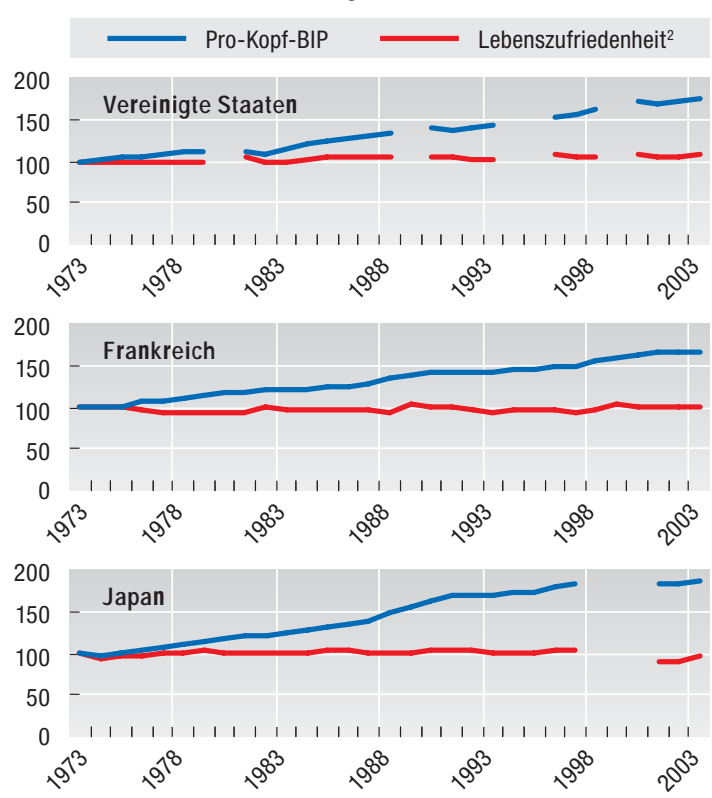

1. Die Angaben über den Grad der Lebenszufriedenheit stammen von Antworten auf die folgende Frage: "Wie zufrieden sind Sie heute alles in allem mit ihrem Leben?" Die durchschnittliche Lebenszufriedenheit wird gemessen als gewichtete Summe zehn unterschiedlicher Zufriedenheitsgrade (von 0 für Personen, die sich als ganz und gar unzufrieden bezeichnen, bis zu 10 für jene, die angeben, rundum zufrieden zu sein), wobei jeder Grad mit dem Anteil der befragten Personen gewichtet wurde, die die betreffende Punktzahl angaben. Das Pro-Kopf-BIP ist in US-\$ zu laufenden KKP von 2000 ausgedrückt.

2. Die Angaben über die Trends der Lebenszufriedenheit beziehen sich auf die Antworten auf Erhebungsfragen nach der Zufriedenheit mit dem Leben allgemein (diese Antworten wurden in zwei Kategorien für die Vereinigten Staaten und in vier Kategorien für die anderen Länder unterteilt).

Quelle: Die Angaben im linken Teil der Abbildung stammen aus den World Values Surveys 1999-2001 und den World Development Indicators der Weltbank (2004); die Daten im rechten Teil der Abbildung wurden der World Database of Happiness entnommen (w w w 2.eur.nl/fsw/research/happiness/trendnat).

Stat Link 2 http://dx.doi.org/10.1787/108105853035

- Was die Vergleiche im Zeitverlauf betrifft, so wurde das Nebeneinander von rasch wachsendem Pro-Kopf-BIP und konstantem Niveau des subjektiven Wohlbefindens als Hinweis darauf gewertet, dass größerer materieller Wohlstand die Menschen nicht zwangsläufig glücklicher macht (vgl. Abb. 2.8, rechter Teil, wo dieser Befund auf der Basis von Daten für ausgewählte OECD-Länder veranschaulicht wird). Die Stabilität des Indikators für das subjektive Wohlbefinden könnte jedoch z.T. darauf zurückzuführen sein, dass bei seiner Messung eine gebundene Variable verwendet wird (d.h. die 
befragten Personen beurteilen ihre Lebenszufriedenheit anhand einer Skala - z.B. von 1 bis 10 -, die im Zeitverlauf konstant bleibt), während das Einkommen mit Hilfe einer ungebundenen Variable (Pro-Kopf-BIP) berechnet wird.

Während die Schlussfolgerungen, die sich aus den aggregierten Daten der einzelnen Länder bezüglich des Zusammenhangs zwischen Einkommen und subjektiver Lebenszufriedenheit ergeben, auch nicht unumstritten sind, besteht auf individueller Ebene größere Klarheit über die Bestimmungsfaktoren von Glücksempfinden und Lebenszufriedenheit.

- Erstens lassen die individuellen Daten zwar einen Zusammenhang zwischen Einkommen und Wohlbefinden erkennen, doch zeigen sie andererseits auch, dass sich die laut eigenen Aussagen bestehenden Differenzen zwischen verschiedenen Personen des subjektiven Wohlbefindens nicht proportional zu den jeweiligen Einkommensunterschieden verhalten. Eine Veränderung des individuellen Einkommens führt nicht zu einer vergleichbaren Veränderung des subjektiven Wohlbefindens, wobei zudem viel von der Richtung der Einkommensänderung abhängt; so hat ein Verlust eine viel einschneidendere Wirkung als ein vergleichbarer Zuwachs. Das liegt vermutlich daran, dass sich die Menschen auf ein bestimmtes Einkommensniveau einstellen ("Tretmühle der Zufriedenheit") und ein höheres Einkommensniveau dann Erwartungen weckt, die schwerer zu erfüllen sind. Ein weiterer Faktor, der den positiven Effekt einer Einkommenserhöhung auf das subjektive Wohlbefinden einschränkt, ist der Wunsch, "mit den Nachbarn mitzuhalten" - obwohl soziale Vergleiche das subjektive Wohlbefinden zuweilen auch erhöhen können.

- Zweitens fallen die Unterschiede beim persönlichen Einkommen in Bezug auf etwaige Differenzen bei der Selbsteinschätzung des Wohlbefindens weniger ins Gewicht als eine Reihe anderer Faktoren, wie z.B. Beruf und Arbeit, familiäre Beziehungen, Gesundheit und Ausbildung sowie Einkommensungleichheit (Di Tella und MacCulloch, 2005). Einige dieser Faktoren sind aber wiederum selbst mit den Differenzen des Pro-Kopf-BIP korreliert.

\section{Schlussfolgerungen}

Alles in allem besteht eine gewisse Kohärenz zwischen den vier Ansätzen zur Messung des Wohlbefindens (Sozialindikatoren, monetäres Einkommen, um verschiedene nichtmarktbestimmte Faktoren berichtigtes monetäres Einkommen sowie subjektive Messgrößen), was aber eine Reihe wichtiger Unterschiede nicht ausschließt. Wenn die Untersuchungen über soziale und subjektive Indikatoren auch noch in den Kinderschuhen stecken, zeigt doch allein schon die Einbeziehung solcher nichtmaterieller Faktoren, dass das monetäre Einkommen nicht der einzige relevante Faktor ist. Diese Untersuchungen bestätigen ferner die Richtigkeit dessen, was der englische Dichter John Donne bereits vor Jahrhunderten so formulierte: "Kein Mensch ist eine Insel, in sich selbst vollständig; jeder Mensch ist ein Stück des Kontinents". Das heißt, dass das Glück des Einzelnen großenteils von der Situation der größeren Gemeinschaft abhängt, in der er lebt, sowie von seinen Beziehungen zu dieser Gemeinschaft. Deshalb werden die in dieser und in den folgenden Ausgaben von Gesellschaft auf einen Blick dargestellten Sozialindikatoren aller Voraussicht nach eine zunehmend wichtige Rolle bei jeder Beurteilung des Wohlergehens des Einzelnen und der Gesellschaft spielen. 


\section{Anmerkungen}

1. Das ist aber natürlich nur eine der Funktionen der OECD-Sozialindikatoren. Neben der Messung des "sozialen Status" in den OECD-Ländern dienen sie dazu, den "sozialen Kontext" und die "Reaktionen der Gesellschaft" auf verschiedene Probleme zu beschreiben (auch kurz "Reaktionsindikatoren" genannt) (vgl. Kap. 1).

2. Dieses Kapitel lehnt sich an die Analyse in Boarini et al. (2006) an.

3. Die vollständige Liste der in sämtlichen Ausgaben von Gesellschaft auf einen Blick veröffentlichten Indikatoren ist Tabelle 1.1 zu entnehmen.

4. Diese Aussage ist noch eindeutiger, wenn die Analyse auf OECD-Länder mit einem Pro-Kopf-BIP von über 25000 US-\$ beschränkt wird; in diesem Fall ist keine der Korrelationen zwischen den Werten der Sozialindikatoren und dem Pro-Kopf-BIP statistisch signifikant.

5. Eine praktische Anleitung zur Konstruktion dieser Gesamtindikatoren finden sich in Hoffman et al. (2005).

6. Der Korrelationskoeffizient zwischen dem (standardisierten) Pro-Kopf-BIP und dem Medianwert des Gesamtindex beträgt 0,76.

7. Die gleiche Berichtigung um die vom Staat und von gemeinnützigen Einrichtungen bereitgestellten Dienste kann auch beim verfügbaren Einkommen der privaten Haushalte vorgenommen werden.

8. Ein umfassendes Konzept für die Entwicklung einer nichtmarktbestimmten Gesamtrechnung in den Bereichen Haushaltsproduktion, Humankapital, Umwelt, Gesundheit und Bildung, Staat und gemeinnütziger Sektor wird beschrieben bei Abraham und Mackie (2005), wo die Ergebnisse eines Expertenpanels des National Research Council for the United States zusammengefasst werden.

9. Die Berücksichtigung der Freizeit nichterwerbstätiger Personen hätte kontroverse Hypothesen über den freiwilligen oder unfreiwilligen Charakter der Erwerbslosigkeit sowie eine Unterscheidung zwischen der Haushaltsproduktion und der Freizeit von Hausfrauen vorausgesetzt. Eine bereits weiter zurückliegende Evaluierung des Effekts von Freizeit (und Einkommensungleichheit) auf das Wohlergehen stammt von Beckerman (1978).

10. Praktische Anregungen zur besseren Integration physikalischer Messgrößen der Umweltbelastung in die Volkswirtschaftlichen Gesamtrechnungen enthält das Handbook of Integrated Environmental and Economic Accounts (eine Gemeinschaftsveröffentlichung von Vereinten Nationen, Europäischer Kommission, IWF, OECD und Weltbank) von 2003. Solche Satellitenkonten stellen in den OECDLändern jedoch keine gängige Praxis dar.

11. Nordhaus und Tobin (1973) ergänzen in ihrem wegweisenden Beitrag über die Messung des wirtschaftlichen Wohlergehens die Aggregate der Volkswirtschaftlichen Gesamtrechnungen um Freizeit, Sicherheitsausgaben und sonstige intermediäre Ausgaben, unbezahlte Hausarbeit sowie einige der verstädterungsbedingten Funktionsstörungen. Sie stellen fest, dass der von ihnen bevorzugte Indikator des auf Pro-Kopf-Basis ermittelten wirtschaftlichen Wohlergehens in den Vereinigten Staaten zwischen 1929 und 1965 um jährlich 1\% zugenommen hat, verglichen mit einer Zuwachsrate von 1,7\% für den privaten Pro-Kopf-Verbrauch und 1,6\% für das Pro-KopfNettosozialprodukt.

\section{Literaturverzeichnis}

Abraham, K.G. und C. Mackie (2005), Beyond the Market - Designing Nonmarket Accounts for the United States, National Research Council of the National Academies, Washington DC.

Atkinson, A.B. (2005), Measurement of Government Output and Productivity for the National Accounts, Atkinson Review: Final Report, Palgrave-MacMillan, Vereinigtes Königreich.

Beckerman, W. (1978), M easures of Leisure, Equality and Welfare, OECD, Paris.

Boarini, R., A. Johansson und M. Mira d'Ercole (2006), "Alternative Measures of Well-being”, OECD Economics Department Working Paper, No. 476, und OECD Social, Employment and Migration Working Paper, No. 33, OECD, Paris.

Di Tella, R. und R. MacCulloch (2005), "Gross National Happiness as an Answer to the Easterlin Paradox?", Economic Working Paper Archive at WUSTLE, April. 
Förster, M. und M. Mira d'Ercole (2005), “Income Distribution and Poverty in OECD Countries in the Second Half of the 1990s", OECD Social, Employment and Migration Working Paper, No. 22, OECD, Paris.

Frey, B.S. und A. Stutzer (2002), Happiness and Economics, Princeton University Press, Princeton und Oxford.

Hoffman, A.E. Giovannini, M. Nardo, M. Saisana, A. Saltelli und S. Tarantola (2005), “Handbook on Constructing Composite Indicators: Methodology and User Guide", OECD Statistics Working Paper, Paris.

Kolm, S.C. (1969), "The Optimal Production of Social Justice”, in J. Margolis und H. Guitton (Hrsg.), Public Economics, MacMillan, London.

Layard, R. (2005), Happiness - Lessons from a New Science, Penguin Press, New York.

Nordhaus, W.D. und J. Tobin (1973), “Is Growth Obsolete?", in M. Moss (Hrsg.), The Measurement of Economic and Social Performance, Studies in Income and Wealth, Vol. 38, National Bureau of Economic Research, Cambridge.

OECD (1986), Living Conditions in OECD Countries - A Compendium of Social Indicators, OECD, Paris.

Sharpe, A. (1999), "A Survey of Indicators of Economic and Social Well-being", Bericht für Canadian Policy Research Networks, Juli. 

TEIL II

\section{OECD-Sozialindikatoren}





\section{Definition und Messung}

Unter den verschiedenen Messgrößen des Systems der Volkswirtschaftlichen Gesamtrechnungen (SNA) ist das Pro-Kopf-Nettonationaleinkommen (NNE) der am besten geeignete Indikator zum Vergleich des wirtschaftlichen Wohlergehens in verschiedenen Ländern. Das Nettonationaleinkommen ist definiert als das Bruttoinlandsprodukt (BIP) zuzüglich Nettoerwerbs- und Vermögenseinkommen aus dem Ausland, abzüglich der Wertminderung des Anlagevermögens (Wohnbauten, Gebäude, Maschinen, Transportmittel und Sachinfrastruktur) durch Verschleiß, Abnutzung und Alterung. Schätzungen des Pro-Kopf-NNE sind jedoch mit größerer Unsicherheit behaftet als solche des Pro-Kopf-BIP, des am häufigsten verwendeten Indikators des Nationaleinkommens (der auch in früheren Ausgaben von Gesellschaft auf einem Blick herangezogen wurde), weil es in der Praxis schwierig ist, die internationalen Einkommensströme und die Wertminderung des Anlagevermögens zu messen. Wegen des Mangels an Daten zur Wertminderung liegen für Ungarn und Polen keine NNE-Schätzungen vor. Gemessen an ihrem Pro-Kopf-Bruttonationaleinkommen (14 000 US-\$ bzw. 11000 US-\$ im Jahr 2003) dürften beide Länder jedoch der unteren Hälfte des in Abbildung GE1.1 aufgezeigten Einkommensspektrums von 10 000-20 000 US-\$ zuzurechnen sein.

Für die Zwecke des Ländervergleichs werden die NNE-Werte in Landeswährung anhand von Kaufkraftparitäten $(K K P)$ in eine gemeinsame Messgröße umgerechnet. Die Kaufkraftparitäten entsprechen dem Betrag in nationaler Währung, der in den einzelnen Ländern erforderlich ist, um den gleichen Waren- und Dienstleistungskorb zu erstehen, der in den Vereinigten Staaten für 1 US-\$ zu haben ist. Die KKP-Schätzungen werden (von OECD und Eurostat gemeinsam) auf der Grundlage eines Vergleichs der Preise von rd. 2500 Posten in verschiedenen Ländern berechnet (Schreyer und Koechlin, 2002). Zur Berechung des Pro-Kopf-NNE wird das NNE durch die Zahl der Einwohner des jeweiligen Landes geteilt, was sowohl die in Privathaushalten als auch die in Einrichtungen lebende Bevölkerung umfasst. Das NNE ebenso wie die KKP unterliegen statistischen Fehlern, weshalb Unterschiede zwischen den Ländern von bis zu $5 \%$ nicht signifikant sind.

Im Durchschnitt der 28 OECD-Länder, für die Daten vorliegen, belief sich das Pro-Kopf-NNE im Jahr 2004 auf rd. 24000 US-\$. In drei Ländern überstieg es in diesem Jahr 30000 US-\$ - in Luxemburg, Norwegen und den Vereinigten Staaten -, während es sich in sechs Ländern unter 20000 US-\$ und in zwei Ländern (Mexiko und der Türkei) unter 10000 US-\$ bewegte (Abb. GE1.1). Im Durchschnitt ist das Pro-Kopf-NNE in den OECDLändern seit 2000 um rd. 15\% gestiegen und um doppelt so viel in Griechenland und der Slowakischen Republik. Über einen längeren Zeitraum betrachtet ging das Wachstum des Pro-Kopf-NNE im OECD-Raum während der siebziger und achtziger Jahre mit einer deutlichen Verringerung der Länderunterschiede einher, in den neunziger Jahren kam diese Konvergenzbewegung jedoch zum Stillstand. In der Gruppe der großen Volkswirtschaften erhöhte sich in Deutschland, Frankreich, Italien und Japan seit Anfang der neunziger Jahre der Abstand gegenüber dem Pro-Kopf-NNE der Vereinigten Staaten (auf 28-35\%), wohingegen das Vereinigte Königreich eine merkliche Verringerung des Gefälles (auf weniger als 20\%) verzeichnete.

OECD-Länder mit höherem Durchschnittseinkommen geben in der Tendenz mehr für den sozialen Schutz aus (öffentliche sowie obligatorische private Ausgaben). Dieser Zusammenhang, der in der Ausgabe 2005 von Gesellschaft auf einen Blick für das Pro-Kopf-BIP belegt wurde, trifft auch für das ProKopf-NNE zu. Besonders eng ist er bei den Gesundheitsausgaben, er lässt sich aber auch bei anderen
Ausgaben feststellen, allerdings in geringerem Umfang. Dafür gibt es mehrere Erklärungen. Bei vielen Sozialausgaben handelt es sich um "Einkommensersatzleistungen", d.h. Transferzahlungen an Personen ohne Erwerbseinkommen oder an ältere Menschen. Je reicher ein Land wird, umso großzügiger werden auch die Transferleistungen. Andere Sozialausgaben dienen de facto dem Kauf von Dienstleistungen bei anderen Akteuren, z.B. von medizinischer Versorgung oder Kinderbetreuung. In dem Maße, wie der Verdienst dieser Dienstleister parallel zu dem der anderen Arbeitskräfte steigt, wachsen auch die Sozialausgaben (Arjona et al., 2001).

Aus diesen Gründen nimmt die Nachfrage nach Sozialausgaben bei wachsendem Nationaleinkommen nicht ab. In der Tat werden die Menschen bei steigendem Einkommen möglicherweise einen größeren Teil davon für den Kauf von sozialen Diensten aufwenden (d.h. die Einkommenselastizität der Sozialausgaben kann größer als 1 sein). Die Daten für die OECD-Länder bestätigen dieses Muster: Der auf Sozialausgaben entfallende Teil des Nationaleinkommens nimmt mit steigendem Pro-Kopf-Einkommen zu, wenngleich hier große Unterschiede zwischen den Ländern bestehen. Dies gilt sowohl für Gesundheitsals auch für andere Sozialausgaben (Abb. GE1.2). Dänemark und Schweden wenden wesentlich mehr für Sozialausgaben auf, als in Anbetracht ihres ProKopf-Einkommens zu erwarten wäre; Mexiko, Korea sowie in geringerem Maße die Vereinigten Staaten geben umgekehrt wesentlich weniger aus. 


\section{GE1.1. Das Nettonationaleinkommen pro Kopf variiert in den OECD-Ländem zwischen 7000 US-\$ und 40000 US-\$}

NNE in US-\$ zu laufenden Preisen und laufenden KKP von 2004

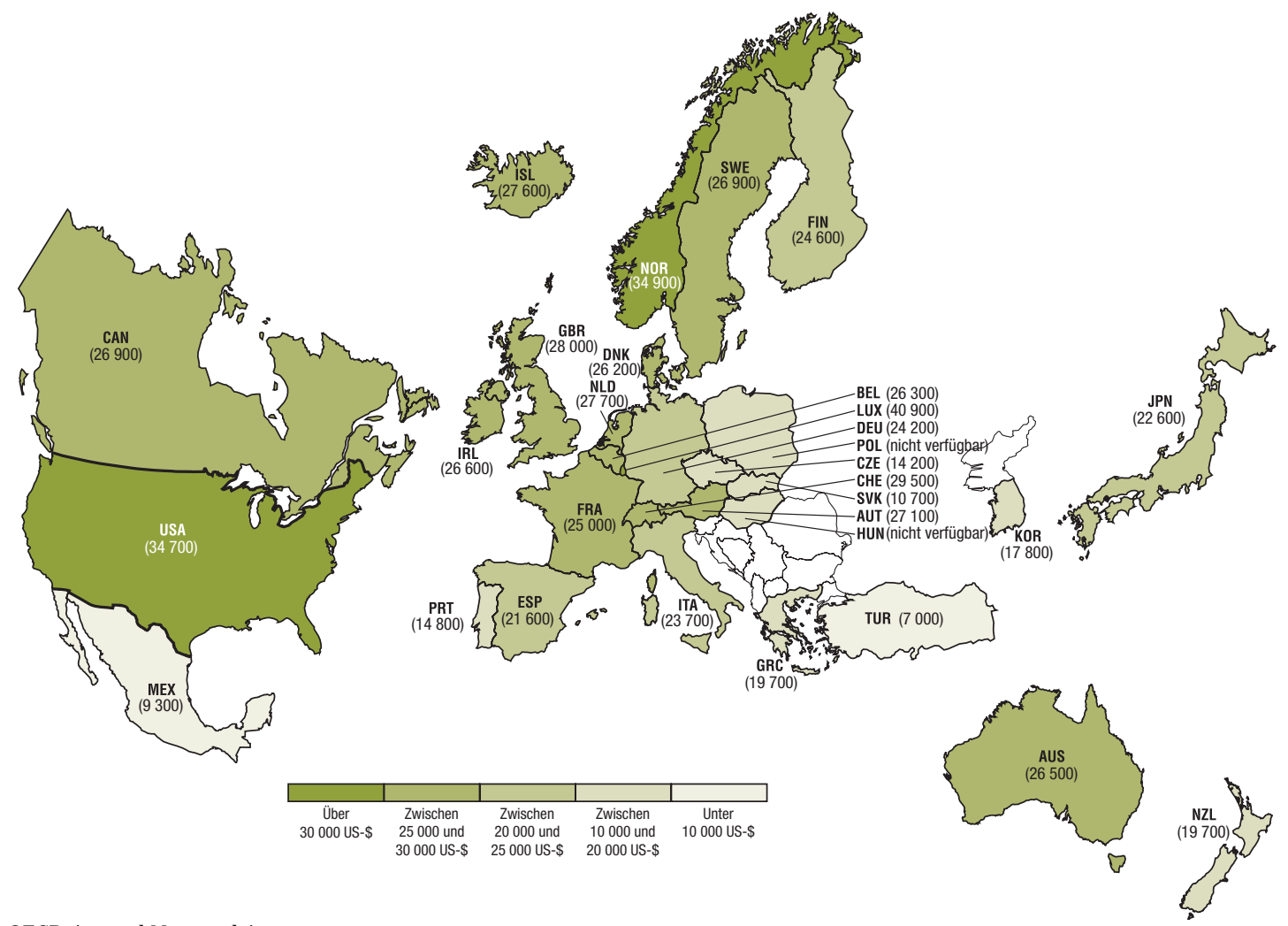

Quelle: OECD Annual National Accounts.

\section{GE1.2. Bei steigendem Pro-Kopf-Einkommen verwenden die OECD-Länder einen größeren Teil ihres Nationaleinkommens für Sozialausgaben}

Pro-Kopf-NNE und Anteil der nicht auf Gesundheit entfallenden Sozialausgaben sowie der gesamten Sozialausgaben am Nationaleinkommen, 2003
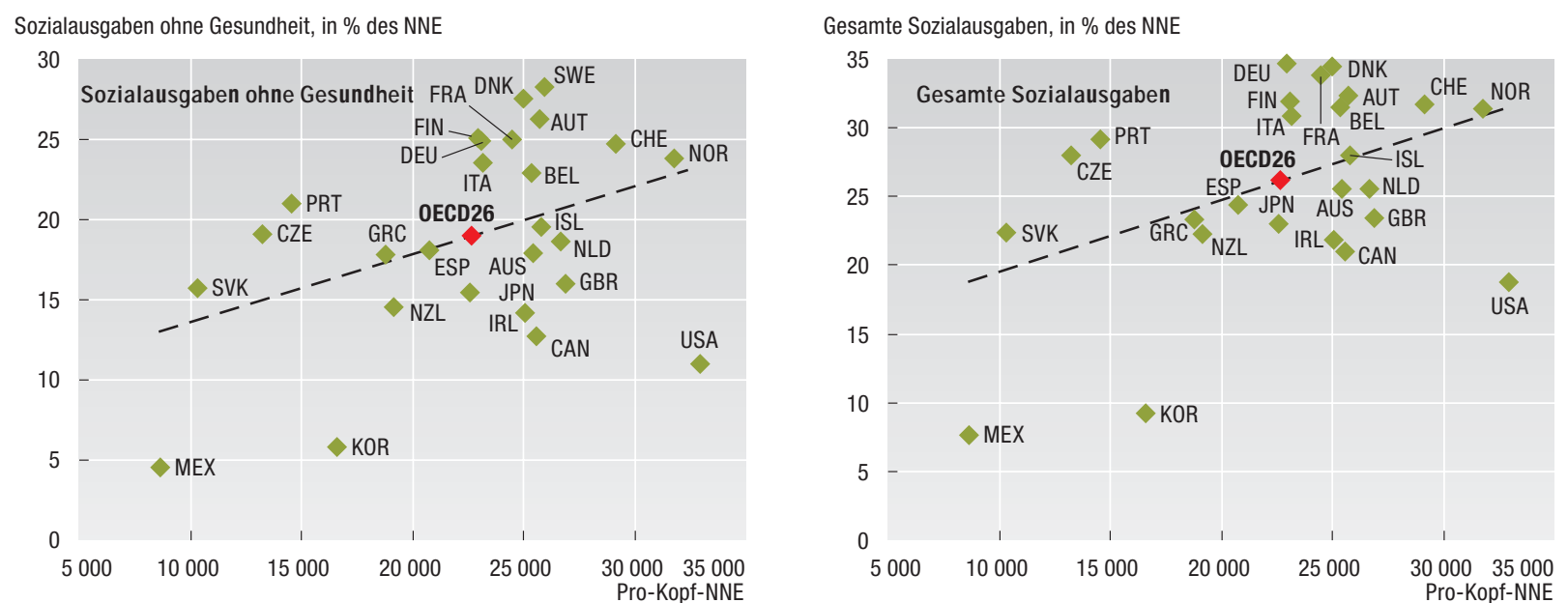

Anmerkung: Die gesamten Sozialausgaben umfassen öffentliche und obligatorische private Ausgaben. Vorläufige Daten.

Quelle: OECD Annual National Accounts und Social Expenditure database.

Weitere Informationen $\square$ Arjona, R., M. Ladaique und M. Pearson (2001), "Growth, Inequality and Social Protection", OECD Labour Market and Social Policy Occasional Paper, No. 51, Paris. OECD (2006), National Accounts of OECD Countries: Main Aggregates, Volume I, 19932004 (2nd version), Paris. — Schreyer P. und F. Koechlin (2002), "Purchasing Power Parities - Measurement and Uses", OECD Statistics Brief, No. 3, März, Paris. 


\section{Definition und Messung}

Die Abhängigenquotienten sind ein Maß der Altersstruktur der Bevölkerung. Zu ihrer Berechnung wird die Zahl der Personen, die in ihrem täglichen Leben normalerweise von der Unterstützung anderer "abhängig" sind - d.h. junge und ältere Menschen -, der Zahl der Personen gegenübergestellt, die diese Unterstützung zu leisten imstande sind. Der nachstehend verwendete Schlüsselindikator setzt die Zahl der Personen unter 20 und ab 65 Jahren zur Zahl der Personen im Alter von 20 bis 64 Jahren in Bezug. In diesem Abschnitt werden zudem zwei weitere Indikatoren vorgestellt, der Jungenquotient (der sich auf die Personen unter 20 Jahren bezieht) und der Altenquotient (der sich auf Personen ab 65 Jahren bezieht), wobei beide Kennziffern im Verhältnis zur Zahl der Personen im Alter von 20 bis 64 Jahren berechnet werden. Zusammengenommen geben diese Quotienten Aufschluss über die in den OECD-Ländern bereits eingetretenen und in Zukunft zu erwartenden demographischen Veränderungen.

Die in diesem Abschnitt verwendeten Projektionen der Abhängigenquotienten stützen sich auf die "mittlere Variante" der jüngsten von den jeweiligen OECD-Ländern erstellten Bevölkerungsvorausberechnungen, wie sie in der OECD Demographic and Labour Force Database erfasst sind. Diese Schätzungen weichen von denen der vorangegangenen Ausgaben von Gesellschaft auf einen Blick ab, die auf den Bevölkerungsprojektionen der Vereinten Nationen basierten.

Die Abhängigenquotienten beeinflussen den globalen Kontext, in dem die Sozialpolitik operiert, und die Art von Bedürfnissen, denen sie gerecht werden muss. Ihre Entwicklung ist eine Funktion von Mortalität, Geburtenziffern und Nettomigration. Eine gestiegene Lebenserwartung und geringere Geburtenziffern führten in allen OECD-Ländern im Zeitverlauf zu einer Zunahme des Altenquotienten und einer Abnahme des Jungenquotienten, wenngleich die erreichten Niveaus und das Ausmaß dieser Entwicklungen in den einzelnen Ländern sehr unterschiedlich waren.

Der Abhängigenquotient reichte 2005 von rd. 55\% in Korea, der Tschechischen Republik und der Slowakischen Republik bis über $80 \%$ in Mexiko und der Türkei, wobei der Durchschnittswert für den OECDRaum insgesamt $65 \%$ betrug (Abb. GE2.1). In Mexiko und der Türkei wird der Abhängigenquotient den Projektionen zufolge sinken (auf rd. 70\%), wohingegen er in allen anderen OECD-Ländern deutlich steigen dürfte; für den OECD-Raum insgesamt wird mit einer Zunahme um 23 Prozentpunkte ( $\mathrm{rd}$. 1/4) gerechnet. Im Jahr 2050 dürfte dieser Quotient in Italien, Japan und Spanien bei über $100 \%$ liegen (d.h. die Zahl der "Abhängigen" wird höher sein als die derjenigen, die für sie sorgen können).

Diese Veränderung des Abhängigenquotienten ist die Folge divergierender Trends an beiden Enden der Altersverteilung. Was die Älteren betrifft, so kamen $2005 \mathrm{im}$ Durchschnitt 24 Menschen im Alter von 65 Jahren und darüber auf 10020 - bis 64-Jährige - um etwa ein Fünftel höheres Verhältnis als 1980.
Zwischen den Ländern bestehen große Unterschiede (Abb. GE2.2, linker Teil): 30-35\% in Deutschland, Griechenland, Italien und Japan gegenüber nur $\mathrm{rd} .10 \%$ in Mexiko und der Türkei. Bis 2050 wird sich der Altenquotient im OECD-Raum insgesamt den Projektionen zufolge mehr als verdoppeln (auf 52\%) und in Mexiko und der Slowakischen Republik verdreifachen. 2050 wird er in Italien, Spanien und Japan bei über 70\% liegen, während er in Dänemark, Island, Luxemburg, Mexiko, der Türkei und den Vereinigten Staaten unter $40 \%$ verharren wird. Die OECD geht davon aus, dass dieser Anstieg des Altenquotienten zu höheren Ausgaben für Gesundheitsversorgung, Langzeitpflege und Renten führen wird. Allein für die Gesundheitsversorgung und die Langzeitpflege könnte sich der Ausgabenanstieg je nach Szenario auf 3,5 bis 6 Prozentpunkte des BIP belaufen (OECD, 2006b).

Demgegenüber sank der Jungenquotient im Durchschnitt der OECD-Länder 2005 auf $41 \%$ - was im Vergleich zu seinem Niveau von 1980 einen Rückgang um 20 Prozentpunkte darstellt - und reichte von 31\% in Italien und Japan bis $70 \%$ oder mehr in der Türkei und Mexiko (Abb. GE2.2, rechter Teil). In den meisten OECD-Ländern wird der Jungenquotient in Zukunft weiter sinken (und $2050 \mathrm{im}$ OECD-Durchschnitt bei $37 \%$ liegen), sich in einer großen Zahl von OECDLändern aber stabilisieren. Der leichte Rückgang des Jungenquotienten könnte zur Senkung der öffentlichen Ausgaben im Bildungsbereich beitragen, diese Abnahme wird jedoch nicht ausreichen, um die höheren Aufwendungen für die ältere Bevölkerung zu kompensieren. 


\section{GE2. ABHÄNGIGENQUOTIENTEN}

GE2.1. Der Abhängigenquotient wird sich in den nächsten 50 Jahren um rd. $1 / 4$ erhöhen

Anteil der Bevölkerung unter 20 und über 64 Jahren in Prozent der Bevölkerung zwischen 20 und 64 Jahren

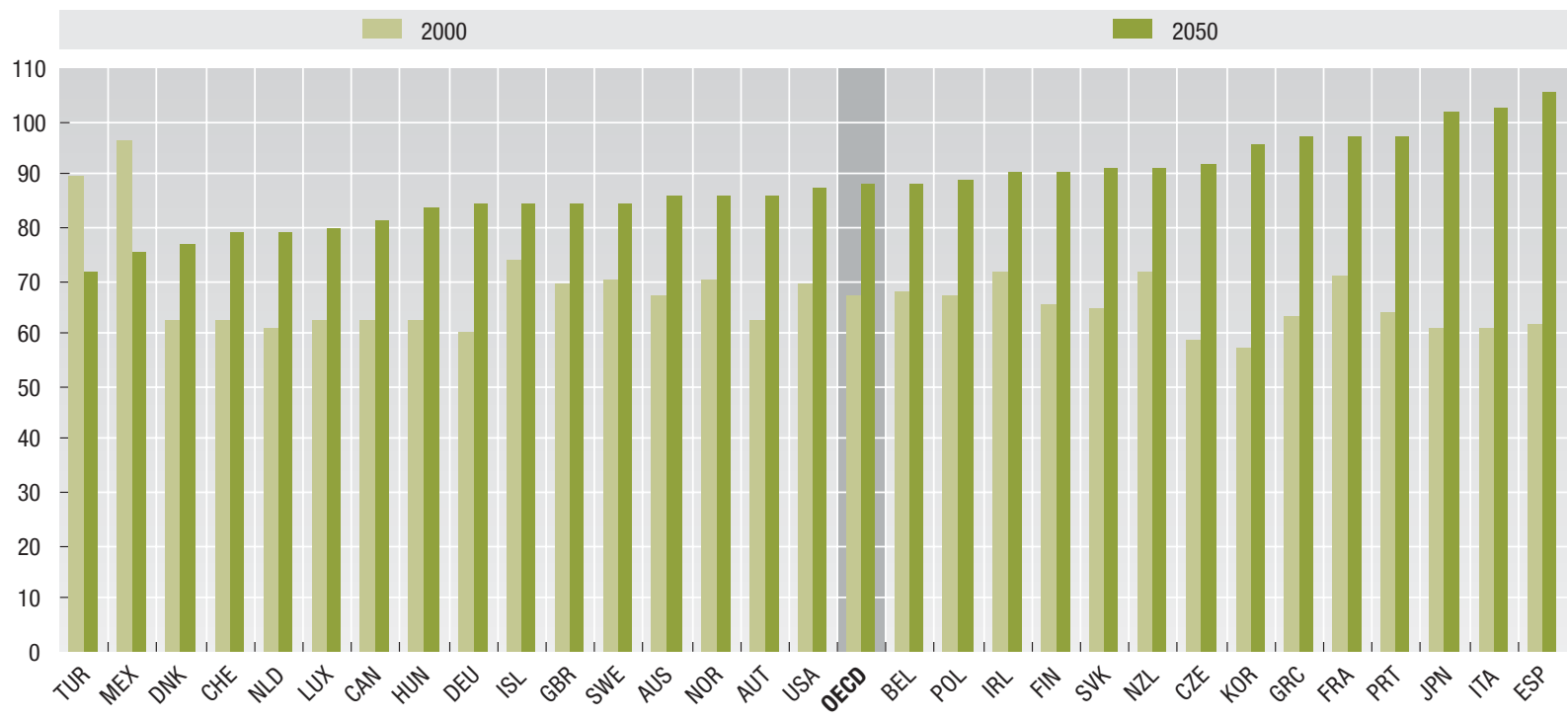

\section{GE2.2. Steiler Anstieg des Altenquotienten und stabile Entwicklung des Jungenquotienten}

A bhängigenquotienten in ausgew ählten OECD-Ländern (Projektionen von 2005)

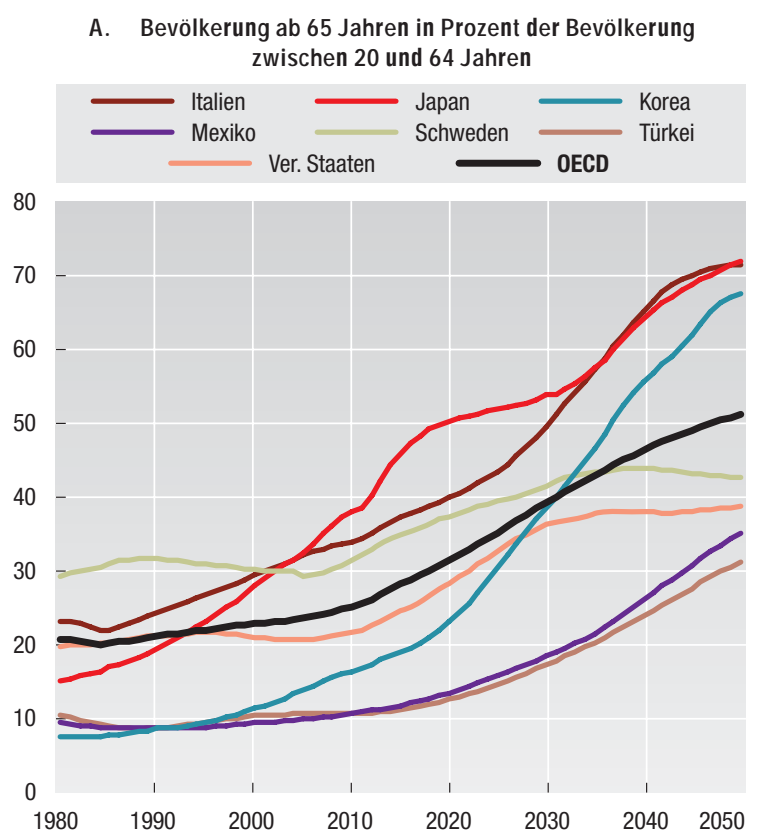

B. Bevölkerung unter 20 Jahren in Prozent der Bevölkerung zwischen 20 und 64 Jahren

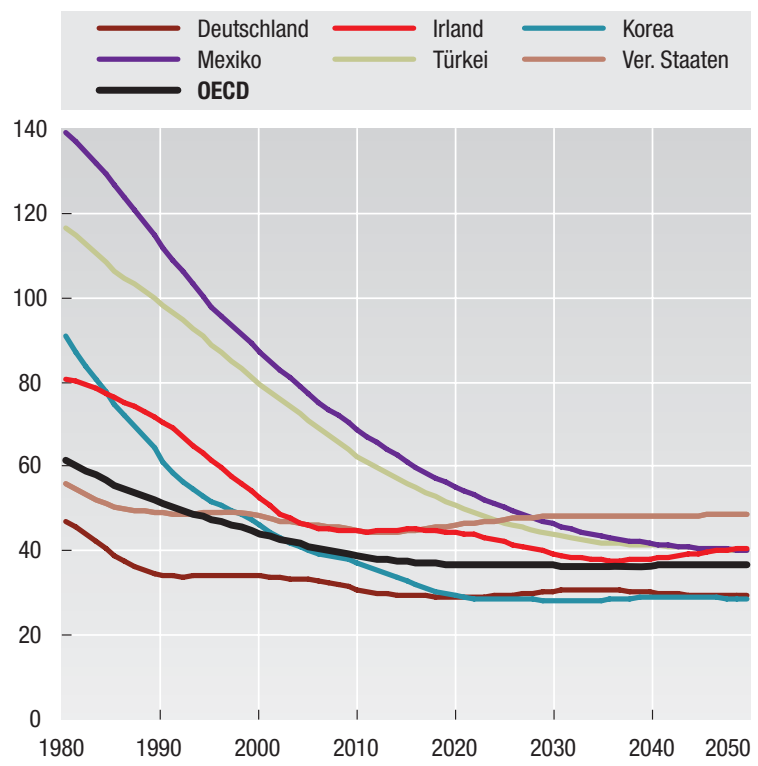

Quelle: OECD Demographic and Labour Force database.

Weitere Informationen $\square$ OECD (2006a), Live Longer, Work Longer, Paris. $\square$ OECD (2006b), “Projecting OECD Health and Long-term Care Expenditures: What are the main drivers?", OECD Economics Department Working Paper, No. 477, Paris. 


\section{Definition und Messung}

Die zusammengefasste Geburtenziffer eines bestimmten Jahres ist die Gesamtzahl der Kinder, die von jeder Frau geboren würden, wenn diese bis zum Ende des gebärfähigen Alters leben und ihre Geburtenwahrscheinlichkeit in jedem Alter der jeweiligen altersspezifischen Geburtenziffer entspräche. Sie wird in der Regel durch Addition der über einen Fünfjahreszeitraum berechneten altersspezifischen Geburtenziffern ermittelt. Bei einer zusammengefassten Geburtenziffer von 2.1 Kindern je Frau bleibt die Bevölkerungszahl ohne Migration und bei unveränderten Sterberaten weitgehend stabil.

Das durchschnittliche Alter bei Geburt des ersten Kindes entspricht dem Alter, in dem die Mütter im Durchschnitt ihr erstes Kind zur Welt bringen, und liefert einen Anhaltspunkt für das Ausmaß der zeitlichen Verschiebung der Mutterschaft. Es errechnet sich aus dem Mittelwert der verschiedenen Altersgruppen, gewichtet mit der Geburtenziffer der jeweiligen Altersgruppen. Der Anteil der nichtehelichen Geburten entspricht der Zahl der in einem bestimmten Jahr außerhalb der Ehe geborenen Kinder im Verhältnis zur Gesamtzahl der Lebendgeburten des gleichen Jahres.

Die zusammengefasste Geburtenziffer liegt in den meisten OECD-Ländern unterhalb des Bestandserhaltungsniveaus, die einzigen Ausnahmen sind Mexiko und die Türkei $(2,2)$ sowie Island und die Vereinigten Staaten (wo sie rd. 2,1 beträgt). 2004 lag die Geburtenziffer im OECD-Durchschnitt bei 1,6, d.h. weit unter dem Niveau, ab dem stabile Bevölkerungszahlen gewährleistet sind; seit 2002 steigt sie aber wieder geringfügig. Hinter diesem Durchschnittswert verbergen sich große Unterschiede zwischen den Ländern wie auch Abweichungen in Bezug auf den Zeitpunkt und das Tempo des Rückgangs der Geburtenziffern. In Japan und Korea sind die Geburtenziffern stark und kontinuierlich gesunken, wohingegen sie in den Vereinigten Staaten und Dänemark (in beiden Fällen ab Mitte der achtziger Jahre) sowie in Frankreich (seit Mitte der neunziger Jahre) wieder gestiegen sind. Seit Anfang dieses Jahrzehnts ist auch in Australien, Deutschland, Italien, Norwegen, Schweden und Spanien erneut ein leichter Anstieg der Geburtenziffern zu beobachten (Abb. GE3.1). Die Gründe für diese Entwicklung sind unterschiedlich, hier machen sich die Einführung spezifischer Maßnahmen in mehreren Ländern wie auch der Effekt der höheren Geburtenziffern der Zuwandererbevölkerung bemerkbar.

Der Rückgang der Geburtenziffern hat eine Reihe negativer Konsequenzen für die Gesellschaft. Dazu gehören ein Verlust an reproduktivem Potenzial, das an der Zahl der Frauen im gebärfähigen Alter gemessen wird, eine Abnahme der Zahl der Personen, die für ältere Familienangehörige sorgen können, eine zunehmende Belastung der Personen im Erwerbsalter, die die Renten und die Gesundheitsversorgung finanzieren müssen, eine ältere und weniger anpassungsfähige Erwerbsbevölkerung sowie eine Abnahme der Inlandsersparnis. Fortgesetzt niedrige Geburtenziffern können auch zur Entstehung von "Niedrigfertilitätsfallen" führen, wenn sich die Geburtenziffern auf einem Niveau von rd. 1,5 oder weniger stabilisieren (Lutz et al., 2005).

Gesunkene Geburtenziffern sind das Resultat von Veränderungen der individuellen Lebensstilpräferenzen und der im Alltagsleben auftretenden Schwierigkeiten, z.B. Arbeitsmarktunsicherheit, Wohnungsnot, zu hohe Kinder- betreuungskosten, wie auch des Unvermögens der Politik, diesen Problemen durch geeignete Formen der Unterstützung zu begegnen. Die positive (und sich vergrößernde) Differenz zwischen der Zahl der Kinder, die die Frauen laut eigenen Angaben haben möchten, und der Zahl der Kinder, die sie tatsächlich zur Welt bringen, lässt darauf schließen, dass diese Probleme ein wichtiger Erklärungsfaktor für den langfristigen Rückgang der Geburtenziffern sind, der in den meisten OECD-Ländern zu beobachten war (D'Addio und Mira d'Ercole, 2005).

Der Trend zu niedrigeren Geburtenziffern ging mit einer Verschiebung des durchschnittlichen Alters bei Geburt des ersten Kindes einher. Das durchschnittliche Alter bei der ersten Geburt ist seit 1970 im Durchschnitt pro Jahrzehnt um ein Jahr gestiegen (Tabelle GE3.2). Abgesehen davon, dass sie zu einem Rückgang der Geburtenziffern führt, hat die Erhöhung des durchschnittlichen Erstgebärendenalters noch andere dauerhafte Konsequenzen: Mit ihr steigt die Wahrscheinlichkeit, dass Frauen kinderlos bleiben oder weniger Kinder haben als sie eigentlich möchten, und vergrößert sich das Morbiditätsrisiko für Mutter und Kind. Etwa die Hälfte der Frauen mit Hochschulabschluss (im Alter von 35 Jahren) ist in Deutschland kinderlos und in der Schweiz sind es unter 40-jährigen Frauen 40\%.

Der Rückgang der Geburtenziffern hängt z.T. auch mit Veränderungen beim Familienstand der Frauen zusammen. In den Ländern, in denen ein starker Zusammenhang zwischen Ehe und Mutterschaft besteht (in Japan, Korea sowie einigen südeuropäischen Ländern) könnte der gestiegene Anteil der unverheirateten Frauen die Geburtenziffern gedrückt haben. Allerdings hat sich auch das Geburtenverhalten der unverheirateten Frauen deutlich verändert. Aus diesem Grund und infolge einer gesunkenen Heiratsneigung werden in den nordischen Ländern heute über die Hälfte aller Kinder nichtehelich geboren, wohingegen dies 1960 nur für jedes zehnte Kind galt. 2004 lag der Anteil der nichtehelichen Geburten in Frankreich bei $47 \%$ und in den Vereinigten Staaten bei $37 \%$. Im Allgemeinen weisen die OECD-Länder mit einem höheren Anteil nichtehelicher Geburten auch höhere Geburtenziffern auf (Abb. GE3.3). 


\section{GE3.1. In den meisten OECD-Ländern liegen die zusammengefassten Geburtenziffern unter dem Bestandserhaltungsniveau}

Zusammengefasste Geburtenziffern von 1970 bis 2004

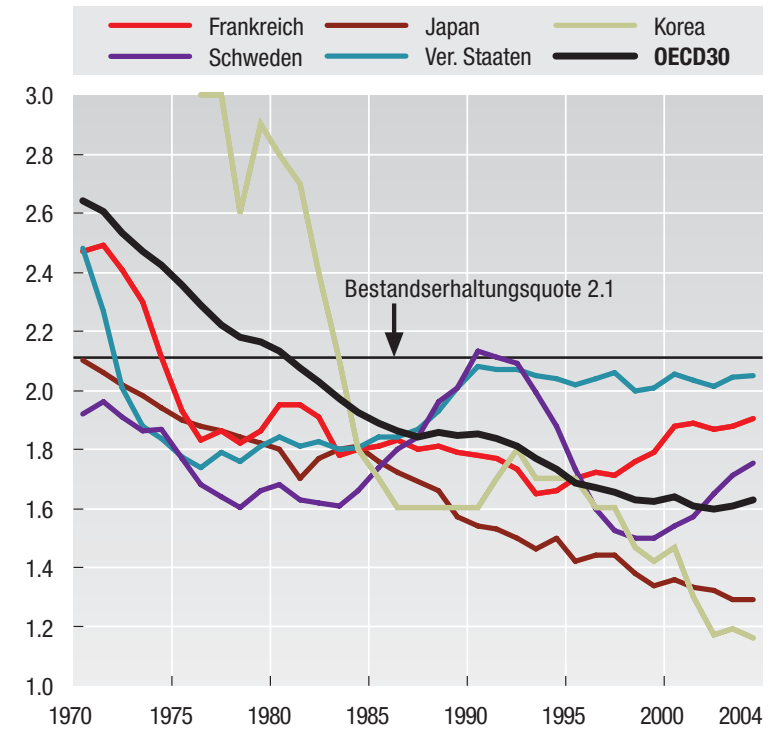

GE3.2. Das Durchschnittsalter bei Geburt des ersten Kindes steigt weiter

\begin{tabular}{|c|c|c|c|c|}
\hline & \multicolumn{4}{|c|}{ Durchschnittsalter bei Geburt des ersten Kindes } \\
\hline & 1970 & 1995 & $2000^{1}$ & $2004^{2}$ \\
\hline Australien & 23.2 & 26.8 & $\ldots$ & . \\
\hline Belgien & 24.3 & 27.3 & $\ldots$ & . \\
\hline Dänemark & 23.8 & 27.4 & 27.7 & 28.4 \\
\hline Deutschland & 24.0 & 27.5 & 28.2 & 29.0 \\
\hline Finnland & 24.4 & 27.2 & 27.4 & 27.8 \\
\hline Frankreich & 24.4 & 28.1 & 27.9 & 28.4 \\
\hline Giechenland & 25.0 & 26.6 & 27.5 & 28.0 \\
\hline Irland & . & 27.3 & 27.6 & 28.5 \\
\hline Island & 21.3 & 25.0 & 25.5 & 26.2 \\
\hline Italien & 25.0 & 28.0 & $\ldots$ & $\ldots$ \\
\hline Japan & 25.6 & 27.5 & 28.0 & 28.9 \\
\hline Korea & $\ldots$ & $\ldots$ & $\ldots$ & 28.9 \\
\hline Luxemburg & 24.7 & 27.4 & 28.4 & 28.6 \\
\hline Neuseeland & 23.5 & 29.2 & 28.0 & 28.0 \\
\hline Niederlande & 24.8 & 28.4 & 28.6 & 28.9 \\
\hline Norwegen & . & 26.4 & 26.9 & 27.6 \\
\hline Polen & 22.8 & 23.8 & 24.5 & 25.6 \\
\hline Portugal & $\ldots$ & 25.8 & 26.5 & 27.1 \\
\hline Österreich & $\ldots$ & 25.6 & 26.4 & 27.0 \\
\hline Schweden & 25.9 & 27.2 & 27.9 & 28.6 \\
\hline Schweiz & 25.3 & 28.1 & 28.7 & 29.3 \\
\hline Slowak. Rep. & 22.6 & 23.0 & 24.2 & 25.3 \\
\hline Spanien & . & 28.4 & 29.1 & 29.2 \\
\hline Tschech. Rep. & 22.5 & 23.3 & 25.0 & 26.3 \\
\hline Ungarn & 22.8 & 23.8 & 25.1 & 26.3 \\
\hline Ver. Königr. & .. & 28.3 & 29.1 & 29.5 \\
\hline Ver. Staaten & 24.1 & 24.5 & 24.9 & 25.1 \\
\hline OECD14 & 23.9 & 26.1 & 26.7 & 27.4 \\
\hline
\end{tabular}

1. 2001 für Neuseeland.

2. 2003 für Finnland, Griechenland, Spanien und Vereinigtes Königreich. 2002 für die Vereinigten Staaten.

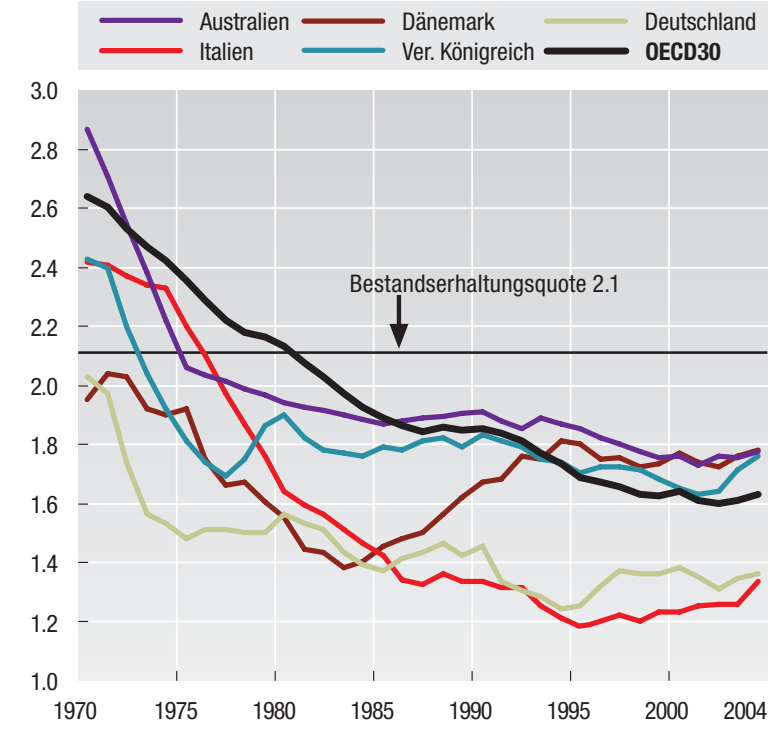

GE3.3. In Ländern mit einem hohen Anteil nichtehelicher Geburten sind auch die Geburtenziffern höher

A nteil der nichtehelichen Geburten und zusammengefasste Geburtenziffer, 2004

Zusammengefasste Geburtenziffer

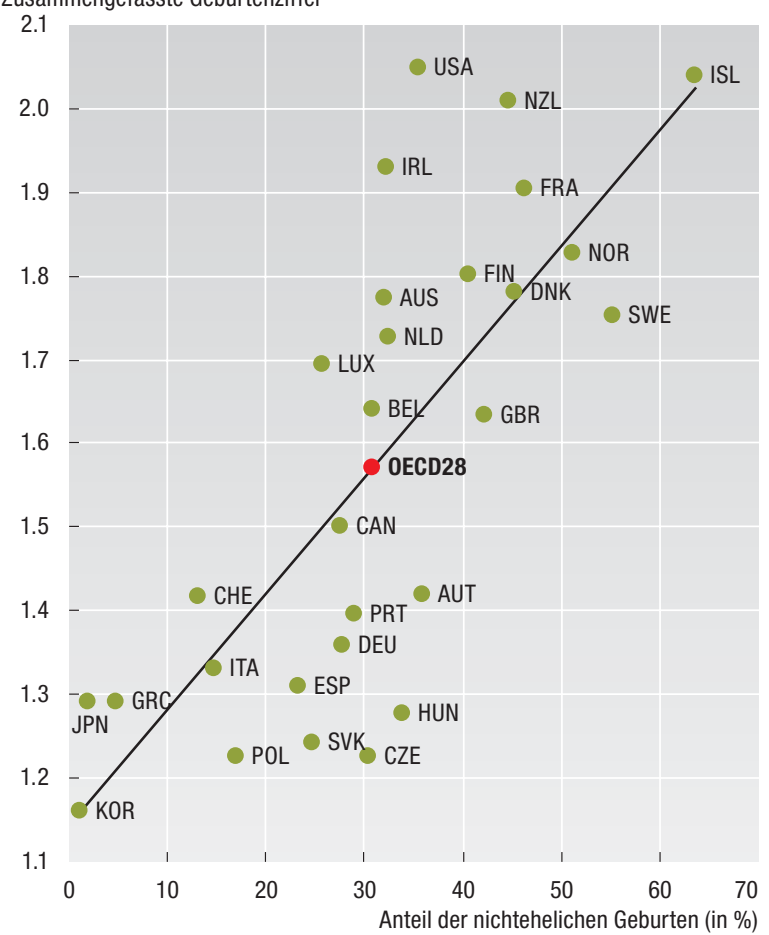

Quelle: Europarat (2006), Recent Demographic Development in Europe, 2004; Eurostat sowie nationale Statistikämter.

$$
\text { StatLink } 2 \text { http://dx.doi.org/10.1787/114055050113 }
$$

Weitere Informationen D'Addio, A.C und M. Mira d'Ercole (2005), “Trends and Determinants of Fertility Rates in OECD Countries: the Role of Policies", OECD Social, Employment and Migration Working Paper, No. 27, Paris. Lutz, W, V. Skirbekk und M.R. Testa (2005), "The Low Fertility Trap Hypothesis", European Demographic Research Papers, No. 01/06, Vienna Institute of Demography, Wien. 


\section{Definition und Messung}

Geburtsland und Staatsangehörigkeit sind die beiden von den OECD-Ländern am häufigsten zur Definition der "zugewanderten" Bevölkerung verwendeten Kriterien. Gemäß dem ersten Kriterium sind Zuwanderer in einem Land ansässige, aber dort nicht geborene Personen, d.h. es handelt sich um Zuwanderer der ersten Generation. Gemäß dem zweiten Kriterium sind Zuwanderer Gebietsansässige, die die Staatsangehörigkeit ihres Herkunftslands besitzen, aber u.U. im Aufnahmeland geboren sind. Im Ländervergleich festzustellende Unterschiede zwischen dem Umfang der im Ausland geborenen Bevölkerung und der ausländischen Bevölkerung hängen mit den jeweils geltenden Regelungen für den Erwerb der Staatsbürgerschaft zusammen. Schätzungen der Zuwandererbevölkerung, die sich auf die im Ausland geborene Bevölkerung stützen, fallen im Allgemeinen wesentlich höher aus als solche, die sich an der Staatsangehörigkeit orientieren. Während die Vergleichbarkeit der Zuwandererzahlen in den verschiedenen Ländern bislang durch Unterschiede in Bezug auf die Definition der Zuwandererbevölkerung beeinträchtigt wurde, liefert diese Ausgabe von Gesellschaft auf einen Blick erstmals international vergleichbare, auf Zensusdaten beruhende Angaben zur im Ausland geborenen Bevölkerung (Dumont und Lemaître, 2005).

Der Wanderungssaldo entspricht der Summe der zuziehenden Ausländer und der zurückkehrenden Migranten in einem gegebenen Jahr, abzüglich der Fortzüge von Aus- und Inländern im selben Jahr. Zwar sind die Zuzugsund Fortzugsdaten im Allgemeinen nicht vergleichbar, in den Statistiken zum Wanderungssaldo, bei dem es sich um die Differenz zwischen Zuzügen und Fortzügen handelt, werden die Hauptursachen der Nichtvergleichbarkeit, d.h. die kurzfristigen Wanderungsbewegungen, aber in der Regel herausgerechnet. Der jährlich erscheinende Internationale Migrationsausblick der OECD liefert eine Gesamtanalyse der jüngsten Trends und Zuwanderungspolitiken in den OECD-Ländern.

Zwischen den OECD-Ländern bestehen große Unterschiede in Bezug auf den Umfang ihrer Zuwandererbevölkerung. Insgesamt waren 2004 rd. 10\% der Bevölkerung des OECD-Raums im Ausland geboren. Am höchsten war dieser Anteil in Luxemburg, der Schweiz und Australien mit über $20 \%$, gefolgt von Neuseeland und Kanada mit fast $18 \%$ sowie den Vereinigten Staaten, Österreich und Deutschland mit rd. 13\% (Abb. GE4.1). Demgegenüber war der Anteil in Mexiko und Polen unerheblich und in der Türkei und Italien lag er bei weniger als $2 \%$.

Die Daten zur im Ausland geborenen Bevölkerung liefern eine vergleichbare Momentaufnahme der Situation zu einem bestimmten Zeitpunkt, ermöglichen jedoch keine Beurteilung der Trends im Zeitverlauf. Dazu sind Daten über Zuzüge und Fortzüge während eines bestimmten Zeitraums, d.h. den Wanderungssaldo, notwendig. Der Wanderungssaldo erhöhte sich - in den 28 in Abbildung GE4.2 dargestellten OECDLändern - in den ersten Jahren dieses Jahrzehnts im Vergleich zu dem Niveau der neunziger Jahre um rund ein Drittel. In einigen Ländern war dieser Anstieg jedoch begrenzt. In Irland, wo traditionell ein Abwanderungsüberschuss in das Vereinigte Königreich und andere klassische Einwanderungsländer verzeichnet wurde, ist seit 1996 ein hoher Zuwanderungsüberschuss zu beobachten, weil das starke Wirtschaftswachstum ehemalige Auswanderer zur Rückkehr bewegt. Südeuropäische Länder wie Italien, Portugal und Spanien entwickelten sich Anfang dieses Jahrzehnts ebenfalls zu Zuwanderungsländern, und auch in Australien, Kanada und Neuseeland wurden höhere Nettozuwanderungsraten verzeichnet. Gering blieb die Nettozuwanderung demgegenüber in der Slowakischen Republik, der Tschechischen Republik und Ungarn, und in Polen war sie negativ (negative Wanderungssalden wurden allerdings einige Jahre lang auch in Island und Japan sowie in jüngerer Zeit in den Niederlanden verzeichnet), während sie in mehreren europäischen Ländern (Dänemark, Niederlande, Finnland, Griechenland, Deutschland und Schweiz) stabil blieb oder zurückging.

Angesichts wachsender Zuwandererströme stehen die OECD-Länder vor der Herausforderung der wirtschaftlichen und sozialen Integration der Migranten. Der Integrationserfolg wird durch die Merkmale der Migranten beeinflusst. Harmonisierte Daten zu den erteilten Aufenthaltsgenehmigungen ermöglichen Vergleiche der Aufschlüsselung der langfristigen Zuwanderer nach Zuwanderungskategorie. Die Daten in Abbildung GE4.3 beziehen sich auf langfristige Zuwanderer, d.h. Personen, denen ein dauerhaftes Niederlassungsrecht eingeräumt wurde, Freizügigkeitsberechtigte (z.B. Bürger anderer EU-Länder) sowie Personen, deren Aufenthaltsgenehmigung mehr oder minder unbegrenzt verlängerbar ist. Die Zusammensetzung dieser langfristigen Zuströme ist von Land zu Land sehr unterschiedlich. Auf Familienangehörige (die im Rahmen des Familiennachzugs oder zusammen mit einem Arbeitsmigranten einreisen) entfiel 2004 der größte Teil der dauerhaften Zuzüge (durchschnittlich rd. 60\%), vor allem in den Vereinigten Staaten und in Schweden (OECD, 2006). Die Aufnahmen aus humanitären Gründen (einschließlich nachziehender Familienmitglieder) sowie sonstigen Motiven (z.B. Abstammung) machten demgegenüber jeweils $10 \%$ aus. Im Durchschnitt handelte es sich bei rd. $20 \%$ aller langfristigen Zuwanderer um Arbeitsmigranten, in Portugal und Dänemark bei fast 50\%. Der Anteil der Arbeitsmigranten hat sich seit Ende der neunziger Jahre deutlich erhöht, nachdem in einigen OECD-Ländern Maßnahmen zur Begrenzung anderer Formen der Zuwanderung sowie (häufig auch) zur Förderung der Zuwanderung qualifizierter Kräfte verabschiedet wurden. Der Anteil der Arbeitsmigranten an den langfristigen Zuzügen stieg im Vereinigten Königreich zwischen 1995 und 2004 von 10\% auf $35 \%$ und in Australien von $20 \%$ auf $32 \%$. 


\section{GE4.1. In Australien, Luxemburg und der Schweiz ist mehr als jeder fünfte Einwohner im Ausland geboren}

Im Ausland geborene Bevölkerung in Prozent der Gesamtbevölkerung, 2004 ${ }^{1}$

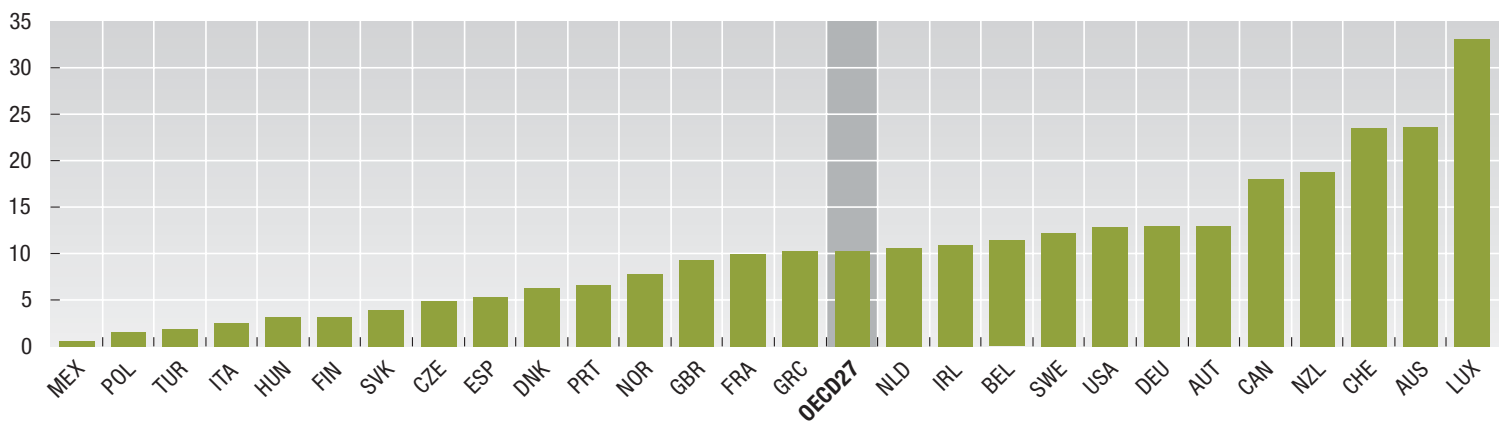

1. Die Daten beziehen sich auf 2004 außer für Frankreich (1999), Mexiko und die Türkei (2000), Griechenland, Italien und Spanien (2001) sowie Portugal (2002). Bei den Daten für Deutschland, Irland, Kanada, Luxemburg, Neuseeland, Portugal, die Schweiz und die Tschechische Republik handelt es sich um Schätzungen der OECD.

\section{GE4.2. Die Wanderungssalden nehmen in den meisten Ländern zu}

JahresdurchschnittlicheWanderungssalden, je1 000 Einw ohner, Durchschnitt 1991-99 (hell) und 2000-04 bzw. letzter vorliegender Zeitraum (dunkel)

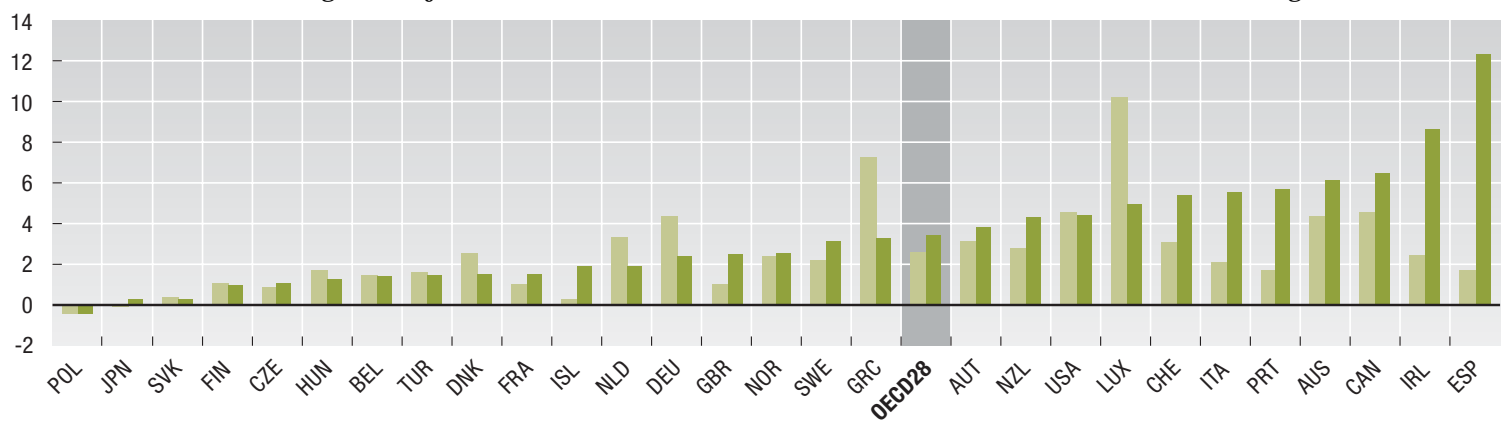

Anmerkung: Die Länder sind in aufsteigender Reihenfolge nach den durchschnittlichen Wanderungssalden im Zeitraum $2000-2004$ dargestellt.

\section{GE4.3. Deutliche Unterschiede bei der Zusammensetzung der langfristigen Zuwandererströme} Aufschlüsselung der langfristigen Zuwandererströme nach Zuwanderungskategorie, 2004, Summe = 100\%

$$
\begin{array}{|ll}
\text { Arbeitsmigranten } & \text { Familienangehörige } \\
\text { Humanitäre Migranten und Angehörige } & \text { Sonstige (ethnische Migranten, Rentner usw.) }
\end{array}
$$

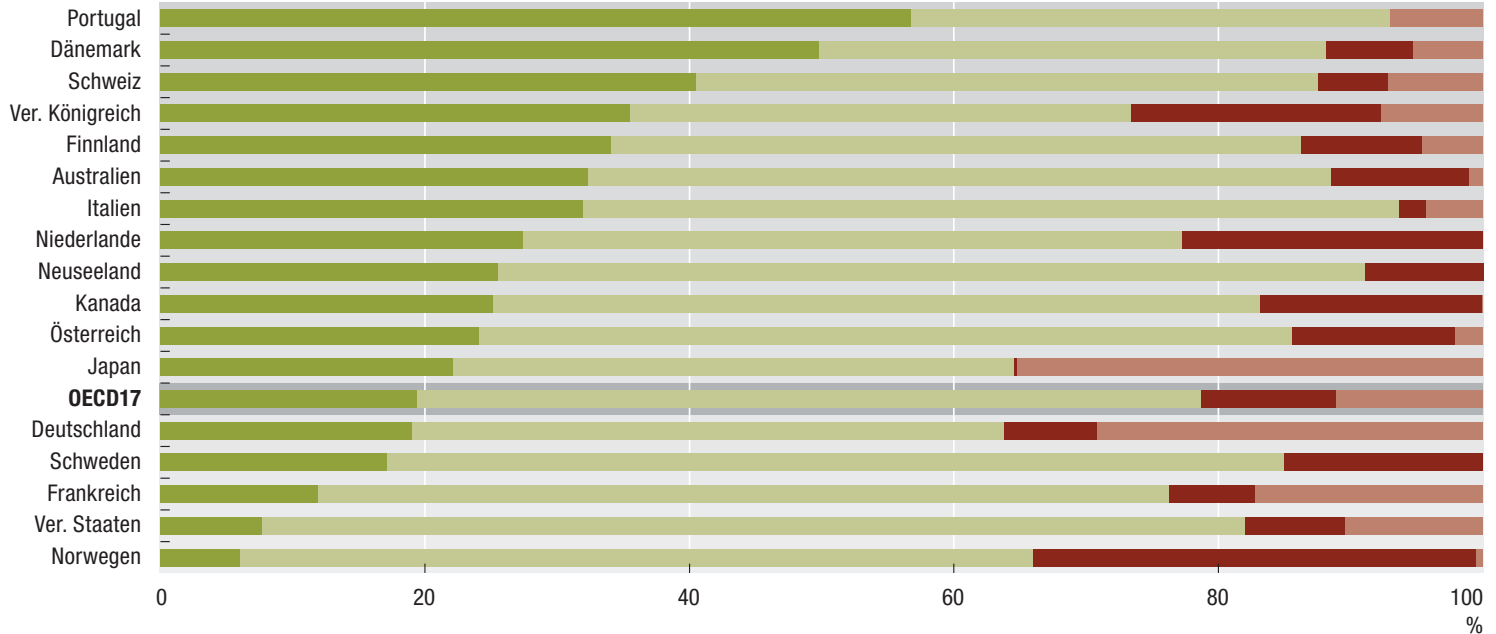

Anmerkung: Die Länder sind in absteigender Reihenfolge nach dem Anteil der Arbeitsmigranten an den Gesamtzuzügen im Jahr 2004 dargestellt. Die Angaben beruhen auf harmonisierten Zahlen, die sich zumeist auf Daten zu den erteilten Aufenthaltsgenehmigungen stützen. In der Gruppe der Familienangehörigen sind sowohl Zuzüge im Rahmen des Familiennachzugs als auch mitreisende Familienangehörige von Arbeitsmigranten erfasst. Quelle: OECD (2006), Internationaler Migrationsausblick, Paris (w ww.oecd.org/els/migration/imo2006).

StatLink $2 \quad$ http://dx.doi.org/10.1787/871482340543

Weitere Informationen Dumont, J-C. und G. Lemaître (2005), “Counting Immigrants and Expatriates in Countries: A New Perspective", OECD Social, Employment and Migration Working Paper, No. 25, Paris. 


\section{Definition und Messung}

Die rohe Heiratsrate entspricht der Zahl der Eheschließungen in einem bestimmten Jahr im Verhältnis zur Gesamtbevölkerung; analog dazu handelt es sich bei der rohen Scheidungsrate um die Zahl der Ehen, die in einem Jahr aufgelöst wurden, im Verhältnis zur Gesamtbevölkerung. In beiden Messgrößen nicht berücksichtigt sind informelle Partnerschaften und andere Formen rechtskräftiger Lebensgemeinschaften (wie sie in einigen OECDLändern in jüngster Zeit eingeführt wurden) wie auch verheiratete, aber getrennt lebende Paare. Alle Angaben - vom Europarat, von Eurostat sowie aus nationalen Quellen - beruhen auf Verwaltungsregistern.

Der nachstehend angegebene Indikator der Rate der nichtehelichen Lebensgemeinschaften misst die Häufigkeit dieser Partnerschaftsformen im Verhältnis zur Zahl der Verheirateten und der Ledigen. Die Daten zu den freien Partnerschaften sind weniger zahlreich und lassen sich unter den verschiedenen OECD-Ländern auch weniger gut vergleichen, was u.a. dadurch bedingt ist, dass diese Lebensgemeinschaften sehr unterschiedliche Formen haben können und der Grad der formellen Anerkennung durch die Rechtssysteme der einzelnen Länder ebenfalls variiert. Die nachstehenden Daten zu den nichtehelichen Lebensgemeinschaften sind nur für die europäischen Länder vergleichbar, wo sie aus der Europäischen Sozialerhebung von 2002 stammen und sich auf die Angaben der Befragten stützen.

Mit wenigen Ausnahmen sind die Eheschließungsraten in allen OECD-Ländern im Zeitraum 1970-2004 gesunken. 2004 lag die rohe Heiratsrate - im Durchschnitt von 26 OECD-Ländern - bei 5,1 je 1000 Einwohner, was das Niveau von 1970 um mehr als ein Drittel unterschritt. Der Rückgang der Heiratsraten vollzog sich in den einzelnen OECD-Ländern in unterschiedlichem Tempo. Besonders ausgeprägt war der Rückgang in Japan und Korea, während Spanien, Dänemark und Schweden seit Ende der neunziger Jahre stabile oder sogar steigende Eheschließungsraten aufweisen (Abb. GE5.1). Parallel zum Rückgang der rohen Heiratsraten war in den meisten OECD-Ländern ein Anstieg der Scheidungsraten zu verzeichnen. 2004 betrug die rohe Scheidungsrate durchschnittlich 2,3 bezogen auf 1000 Einwohner, und war damit doppelt so hoch wie 1970 und um 0,2 Prozentpunkte höher als 2000. Im Zeitraum 1999-2003 waren die Scheidungsraten in Kanada, den Vereinigten Staaten, Finnland, Schweden, der Schweiz und den Niederlanden stabil oder rückläufig, wohingegen sie in Spanien und Korea weiter stiegen (Abb. GE5.2). Der Rückgang der Heiratsraten ging mit einer Tendenz zu einem späteren Heiratsalter einher. Das durchschnittliche Alter der Frauen bei der ersten Heirat erhöhte sich zwischen 1990 und 2002-2003 von 24,8 auf 27,7 Jahre, wobei dieser Anstieg in Ungarn, der Tschechischen Republik und Island stärker ausfiel (Abb. GE5.3).

Die Entscheidung zu heiraten oder sich scheiden zu lassen hängt sowohl von den Merkmalen der Einzelnen als auch der Gesellschaft ab. Wer wirtschaftlich gut gestellt ist, heiratet mit größerer Wahrscheinlichkeit und bleibt auch mit größerer Wahrscheinlichkeit verheiratet (Smock, 2004). Die Heiratsentscheidung ist aber auch davon abhängig, ob die Betreffenden überzeugt sind, dass ihre wirtschaftliche Situation eine dauerhafte Ehe ermöglicht. Die Ehe gilt daher häufig als eine Lebensphase, in die man erst eintritt, wenn eine gewisse wirtschaftliche Sicherheit erreicht ist (z.B. Wohneigentum, finanzielle Stabilität usw.). Der gesellschaftliche Kontext hat auch
Einfluss auf die Entscheidung, sich scheiden zu lassen. Zu den Faktoren, die hier ins Gewicht fallen, gehört die bessere finanzielle Stellung erwerbstätiger Frauen, die zunehmende Sensibilisierung für den Konflikt zwischen der Rolle der Frau in Familie und Beruf sowie die geringere Stigmatisierung im Fall einer Scheidung. Im Ländervergleich festzustellende Unterschiede bei den Scheidungsraten können auch auf unterschiedliche rechtliche Vorschriften bezüglich der Zeit bis zur Aussprechung einer Scheidung (die von null in den Niederlanden sowie einigen osteuropäischen Ländern bis zu vier Jahren in Irland und Griechenland reichen kann), besondere Regeln für Scheidungen im beiderseitigen Einvernehmen sowie Bestimmungen, die eine Eheberatung vorschreiben, zurückzuführen sein. In einigen Ländern (z.B. Polen) ist eine Scheidung nur möglich, wenn ein Verschulden seitens eines der Ehepartner vorliegt.

Das Auseinanderbrechen einer Familie kann negative Auswirkungen auf die Kinder haben, insbesondere wenn sie noch klein sind. Dazu gehören ein größeres Risiko finanzieller Armut, schlechtere schulische Leistungen sowie eine höhere Scheidungswahrscheinlichkeit im späteren Leben (Diekmann und Schmidheiny, 2006). Der Kausalzusammenhang lässt sich hier allerdings schwer nachweisen, weil die schlechteren Entwicklungsergebnisse von Kindern geschiedener Eltern auf nichtbeobachtete Faktoren zurückzuführen sein könnten; einige Untersuchungen lassen in der Tat darauf schließen, dass es für die Kinder besser ist, getrennte Eltern zu haben, als ständigen Konflikten zwischen den Eltern ausgesetzt zu sein (Morrison und Coiro, 1999).

Parallel zum Rückgang der Eheschließungsraten war eine Zunahme der Häufigkeit anderer Lebensgemeinschaften zu beobachten. In den europäischen Ländern gaben durchschnittlich rd. 8\% der Befragten an, in einem eheähnlichen Verhältnis zu leben, wobei dieser Prozentsatz in den nordischen Ländern sowie in der Altersgruppe der 20- bis 40-Jährigen wesentlich höher war (Tabelle GE5.4). 


\section{GE5.1. Starke Abnahme der rohen Heiratsrate in vielen OECD-Ländern}

Eheschließungen je 1000 Einw ohner, 1970-2004

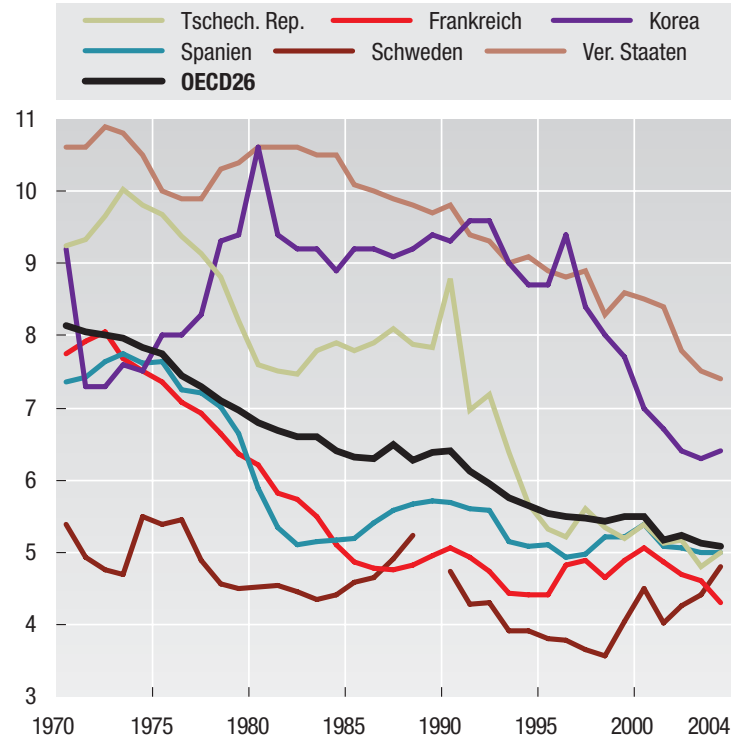

GE5.3. Das Alter bei der ersten Heirat ist gestiegen

Durchschnittsalter der Frauen bei der ersten Heirat, 1990 (heller Farbton) und 2004 (dunkler Farbton)

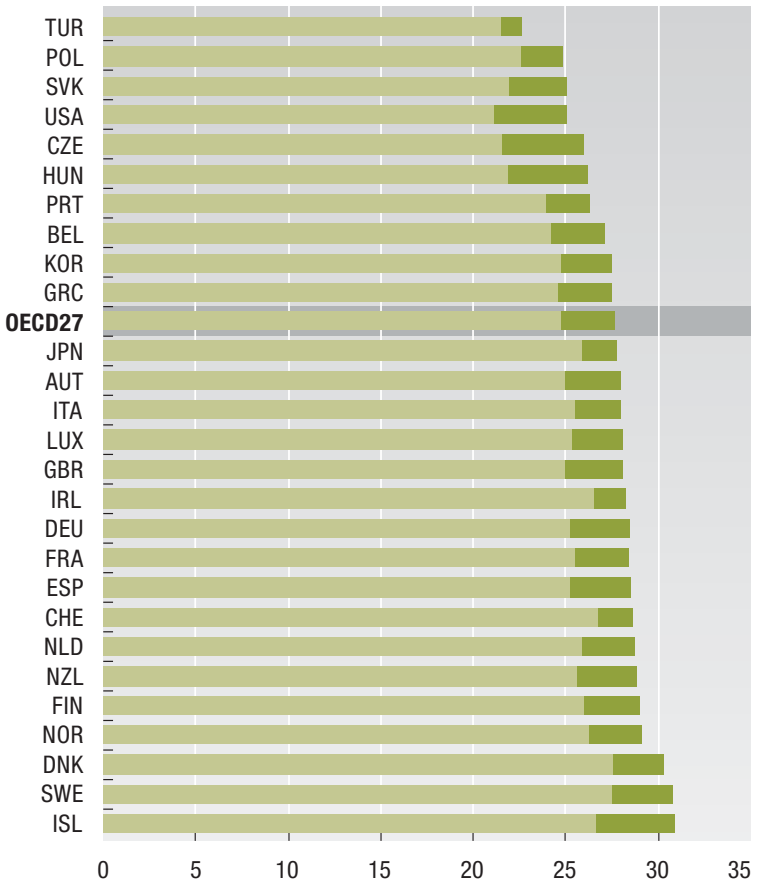

Quelle: Eurostat NewCronos; Europarat: Recent Demographic Development in Europe; nationale Statistikämter.

\section{GE5.2. Allmählicher Anstieg} der rohen Scheidungsrate

Ehescheidungen je 1000 Einw ohner, 1970-2004

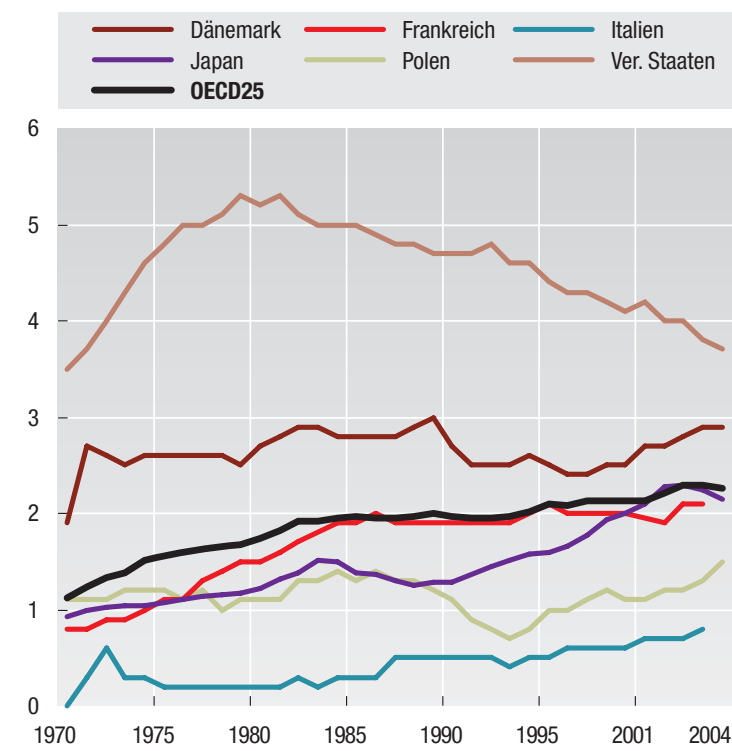

GE5.4. Hohe Prävalenz nichtehelicher Lebensgemeinschaften und anderer Partnerschaftsformen

A nteil der verheirateten, der unverheiratet zusammenlebenden und der alleinstehenden Erwachsenen, 2002

\begin{tabular}{lrrr|rrr}
\hline & \multicolumn{3}{c|}{ Alle Altersgruppen } & \multicolumn{3}{c}{ 20- bis 40-Jährige } \\
\cline { 2 - 7 } & Verheiratet Zusammen- & $\begin{array}{c}\text { Allein- } \\
\text { lebend }\end{array}$ & Stehend & Verheiratet & $\begin{array}{c}\text { Zusammen- } \\
\text { lebend }\end{array}$ & $\begin{array}{c}\text { Allein- } \\
\text { stehend }\end{array}$ \\
\hline Belgien & 52.4 & 7.4 & 40.2 & 42.1 & 13.6 & 44.4 \\
Dänemark & 54.1 & 15.8 & 30.1 & 36.7 & 30.6 & 32.7 \\
Deutschland & 54.8 & 9.6 & 35.6 & 44.6 & 18.7 & 36.7 \\
Finnland & 49.4 & 12.1 & 38.5 & 36.5 & 26.8 & 36.7 \\
Frankreich & 50.7 & 10.7 & 38.6 & 43.2 & 20.9 & 35.9 \\
Griechenland & 61.2 & 1.2 & 37.7 & 54.9 & 2.6 & 42.5 \\
Irland & 55.1 & 3.7 & 41.3 & 41.7 & 7.7 & 50.6 \\
Italien & 59 & 2.8 & 38.2 & 43.7 & 3.6 & 52.7 \\
Luxemburg & 52.7 & 6.1 & 41.2 & 48.2 & 12 & 39.8 \\
Niederlande & 56.4 & 9.2 & 34.3 & 53.7 & 19.5 & 26.8 \\
Norwegen & 50.9 & 18.1 & 31.1 & 32.1 & 34.8 & 33.2 \\
Österreich & 50.7 & 9.4 & 40 & 44 & 15.3 & 40.8 \\
Polen & 56.6 & 1.7 & 41.8 & 57.7 & 2.9 & 39.4 \\
Portugal & 59.1 & 2.1 & 38.8 & 57.6 & 3.6 & 38.8 \\
Schweden & 45.5 & 19.8 & 34.7 & 28 & 35.4 & 36.6 \\
Schweiz & 51.7 & 9.9 & 38.5 & 48.9 & 14.7 & 36.4 \\
Spanien & 56 & 2.1 & 41.9 & 51.3 & 3.2 & 45.5 \\
Tschech. Rep. & 53.9 & 4.1 & 42 & 53.6 & 6.1 & 40.3 \\
Ungarn & 54.8 & 6.7 & 38.5 & 49.6 & 10.8 & 39.6 \\
Ver. Königreich & 47.7 & 8.6 & 43.7 & 38.8 & 17.6 & 43.5 \\
OECD20 & 53.6 & 8.1 & 38.3 & 45.3 & 15.0 & 39.6 \\
\hline Ouenyyyyyyyy & & & & & & \\
\hline
\end{tabular}

Quelle: Hamplová, D. (2005), “Educational Homogamy in Marriage and Cohabitation in Selected European Countries", Treffen des International Sociological Association Research Comittee 28 on Social Stratification and Mobility zum Thema "Inequality and Mobility in Family, School and Work", Los Angeles, 18.-21. August.

StatLink 2 http://dx.doi.org/10.1787/436105386284

Weitere Informationen Diekmann, A. und K. Schmidheiny (2006), "The Intergenerational Transmission of Divorce - A FifteenCountry Study with the Fertility and Family Survey", Arbeitsdokument für das 2004 Annual Meeting of the Population Association of America. Morrison, D. und M. Coiro (1999), "Parental Conflict and Marital Disruption: Do Children Benefit When High-Conflict Marriages are Dissolved?", Journal of Marriage and the Family, Vol. 61. " Smock, P. (2004), "The Wax and Wane of Marriage: Prospects for Marriage in the 21st Century", Journal of Marriage and the Family, Vol. 66. 




\section{Definition und Messung}

Nach der Definition der Internationalen Arbeitsorganisation gilt eine Person als "erwerbstätig", wenn sie mindestens eine Stunde pro Woche gegen Entgelt oder im Rahmen einer selbstständigen oder mithelfenden Tätigkeit (gegen Geld- oder Sachleistung) arbeitet oder sich in einem Arbeitsverhältnis befindet, das sie wegen Krankheit, Urlaub oder Arbeitskonflikten vorübergehend nicht ausübt. Die in diesem Abschnitt verwendeten Daten aus Arbeitskräfteerhebungen der OECD-Länder basieren auf dieser Abgrenzung. Der hier für die Beschäftigung herangezogene Basisindikator ist das Verhältnis zwischen Beschäftigung und Bevölkerung (auch Erwerbstätigenquote genannt). Diese Quote gibt den Anteil der selbstständig oder abhängig Beschäftigten an der Bevölkerung im erwerbsfähigen Alter (Personen im Alter von 15 bis 64 Jahren) wieder. Die Erwerbstätigenquoten sind nach Alter, Geschlecht und Bildungsabschluss aufgeschlüsselt.

Dieser Abschnitt enthält auch Daten über die Inzidenz der Teilzeitbeschäftigung in Prozent der Gesamtbeschäftigung. Der Begriff Teilzeiterwerbstätigkeit bezieht sich auf Personen, die gewöhnlich weniger als 30 Wochenstunden an ihrem Hauptarbeitsplatz verbringen, und die Daten erfassen nur Personen, die Fragen zu ihrer normalen Arbeitszeit beantworteten. Die OECD-Daten über Beschäftigung stützen sich auf nationale Arbeitskräfteerhebungen.

Die Erwerbstätigenquoten unter der Bevölkerung im erwerbsfähigen Alter sind in den meisten OECD-Ländern seit Mitte der neunziger Jahre gestiegen. In vielen OECD-Ländern bewegten sich die Erwerbstätigenquoten 2005 auf ihrem höchsten Stand seit den achtziger Jahren (Abb. SS1.1). Die Zunahme der Erwerbstätigenquoten betrug in Finnland, Griechenland, Italien, Kanada und den Niederlanden mehr als 5 Prozentpunkte und in Irland und Spanien über 10 Prozentpunkte. Demgegenüber liegen die Erwerbstätigenquoten in mehreren mittel- und osteuropäischen Ländern wie auch in der Türkei um etwa 5 Prozentpunkte unter ihrem Niveau von Mitte der neunziger Jahre. Nach wie vor sind bei den Erwerbstätigenquoten beträchtliche Unterschiede zwischen den Ländern auszumachen, wobei die Spanne von $70 \%$ oder mehr in den Vereinigten Staaten, Australien, den Niederlanden, Kanada, dem Vereinigten Königreich und Schweden bis zu $60 \%$ oder weniger in der Türkei, Polen, Ungarn, Italien, der Slowakischen Republik und Mexiko reicht (Tabelle SS1.3).

Vom allgemeinen Anstieg der Erwerbstätigenquoten haben nicht alle Gruppen gleichermaß en profitiert. Jugendliche, Frauen, ältere und weniger qualifizierte Arbeitskräfte sehen sich bei der Arbeitsuche nach wie vor größeren Schwierigkeiten gegenüber als Männer im besten Erwerbsalter. Die Erwerbstätigenquoten im Ausland geborener Personen sind, vor allem bei Frauen, ebenfalls viel niedriger als die der im Inland geborenen Personen (OECD, 2006). Der Anteil junger Menschen (zwischen 15 und 24 Jahren) in bezahlter Beschäftigung ist im Zeitverlauf stetig zurückgegangen, was sich vor allem aus dem längeren Verbleib im Bildungssystem erklärt. Im Durchschnitt ist die Erwerbstätigenquote junger Menschen um ein Drittel niedriger als die der Personen im Haupterwerbsalter, und in Luxemburg, der Tschechischen Republik, Frankreich, Ungarn und Belgien beträgt der Unterschied über 50\%. Die Erwerbstätigenquoten älterer Menschen (zwischen 55 und 64 Jahren) liegen im Durchschnitt ebenfalls 25 Prozentpunkte unter denen der Personen im besten Erwerbsalter. Eine effizientere Mobilisierung des potenziellen Arbeitsangebots älterer Menschen wird bei der Bewältigung der Bevölkerungsalterung in Zukunft eine ganz entscheidende Rolle spielen.
Die Erwerbstätigenquoten der Frauen blieben 2005 um 16 Prozentpunkte hinter denen der Männer zurück. In der Türkei, Mexiko, Italien, Griechenland und Polen erreichten sie weniger als 50\%, in der Schweiz, Dänemark, Schweden, Norwegen und Island dagegen über 70\%. In zahlreichen Ländern (Dänemark, Kanada, Norwegen, Island, Schweden und Finnland) beträgt der Abstand bei den Erwerbstätigenquoten von Männern und Frauen im Haupterwerbsalter weniger als 10 Prozentpunkte. Im Durchschnitt liegen die Erwerbstätigenquoten von Personen ohne SekundarstufeII-Abschluss um 17 Prozentpunkte unter denen von Personen mit einem solchen Abschluss und um 27 Punkte unter denen von Personen mit Hochschul- oder sonstigem Tertiärabschluss.

Höhere Erwerbstätigenquoten gingen in mehreren OECD-Ländern mit einer größeren Inzidenz der Teilzeitbeschäftigung einher, insbesondere in Belgien, Deutschland, Irland, Italien, Japan und den Niederlanden (OECD, 2006). Die Bedeutung der Teilzeitarbeit ist in den einzelnen OECDLändern recht unterschiedlich (Abb. SS1.2). Im Allgemeinen ist sie unter älteren und jüngeren Arbeitskräften sowie Frauen stärker verbreitet. In elf OECD-Ländern übersteigt die Inzidenz der Teilzeitbeschäftigung unter der Bevölkerung im erwerbsfähigen Alter 30\%, hierzu zählen u.a. Australien, Dänemark, Japan, Kanada, die Niederlande und das Vereinigte Königreich; unter älteren Arbeitskräften liegt sie in Japan, den Niederlanden und dem Vereinigten Königreich nahe an diesem Niveau. Für jüngere Menschen ist Teilzeitbeschäftigung eine Möglichkeit, Arbeit und Ausbildung miteinander zu kombinieren. Ebenso können Teilzeitbeschäftigungen es Frauen erleichtern, berufliche und familiäre Pflichten miteinander zu vereinbaren.

Statusindikatoren: Arbeitslosigkeit (SS2), Erwerbstätige Mütter (SS3), Lohngefälle zwischen Männern und Frauen (EQ3).

Reaktionsindikatoren: Steuer- und Abgabenkeil auf den Faktor Arbeit (SS5), Leistungen bei Erwerbslosigkeit (SS6). 


\section{SS1.1. Im Durchschnitt sind mehr Menschen in Beschäftigung}

Erwerbstätigenquote, Personen zwischen 15 und 64 Jahren

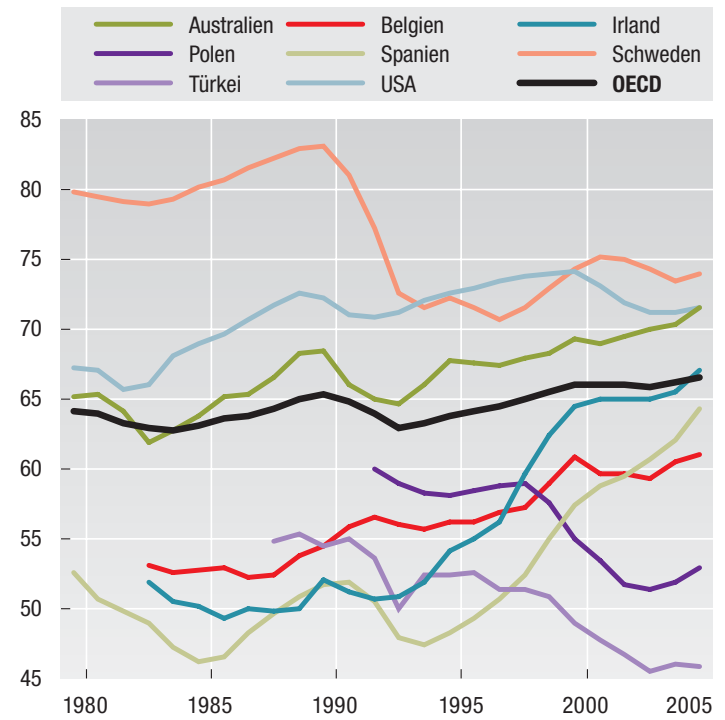

\section{SS1.2. Höhere Inzidenz der Teilzeitbeschäftigung} unter älteren und unter jungen Menschen

Inzidenz der Teilzeitbeschäftigung, nach Altersgruppe, $2005^{1}$

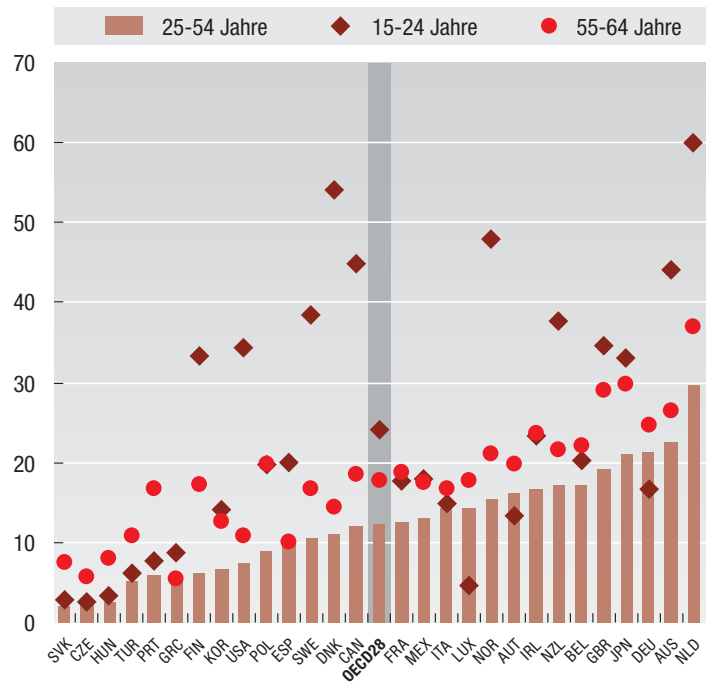

1. 2004 für Mexiko und Schweden.

SS1.3. Beschäftigungsindikatoren, $2005^{1}$

\begin{tabular}{|c|c|c|c|c|c|c|c|c|c|c|c|c|}
\hline & \multirow{3}{*}{$\begin{array}{c}\text { Erwerbstätigen- } \\
\text { quote für die } \\
\text { Bevölkerung } \\
\text { zwischen } 15 \\
\text { und } 64 \text { Jahren }\end{array}$} & \multicolumn{8}{|c|}{ Erwerbstätigenquote nach: } & \multirow{2}{*}{\multicolumn{3}{|c|}{$\begin{array}{l}\text { Inzidenz der Teilzeitbeschäftigung } \\
\text { in \% der Beschäftigung }\end{array}$}} \\
\hline & & \multicolumn{3}{|c|}{ Alter } & \multicolumn{2}{|c|}{ Geschlecht } & \multicolumn{3}{|c|}{ Bildungsabschluss } & & & \\
\hline & & $15-24$ & $25-54$ & $55-64$ & Männer & Frauen & $\begin{array}{c}\text { Ohne } \\
\text { Sekundar- } \\
\text { stufell- } \\
\text { Abschluss }\end{array}$ & $\begin{array}{l}\text { Sekundar- } \\
\text { stufe-II- } \\
\text { Abschluss }\end{array}$ & $\begin{array}{c}\text { Tertiär- } \\
\text { abschluss }\end{array}$ & Insgesamt & Männer & Frauen \\
\hline Australien & 71.6 & 63.6 & 78.8 & 53.7 & 78.5 & 64.7 & 60.6 & 78.8 & 83.3 & 27.3 & 15.7 & 41.7 \\
\hline Belgien & 61.0 & 26.6 & 78.3 & 32.1 & 67.7 & 54.1 & 49.4 & 73.1 & 84.1 & 18.1 & 6.2 & 33.1 \\
\hline Dänemark & 75.5 & 62.0 & 83.9 & 59.8 & 80.1 & 70.8 & 62.0 & 79.7 & 86.5 & 18.0 & 12.0 & 24.9 \\
\hline Deutschland & 65.5 & 42.6 & 77.4 & 45.5 & 71.4 & 59.6 & 48.6 & 69.5 & 82.7 & 21.8 & 7.4 & 39.4 \\
\hline Finnland & 68.0 & 39.2 & 81.7 & 52.6 & 69.4 & 66.5 & 57.0 & 74.2 & 84.5 & 11.2 & 7.9 & 14.8 \\
\hline Frankreich & 62.3 & 26.0 & 79.6 & 40.7 & 67.8 & 56.9 & 59.6 & 75.4 & 81.7 & 13.6 & 5.3 & 23.3 \\
\hline Giechenland & 60.3 & 25.3 & 74.3 & 41.6 & 74.5 & 46.2 & 56.8 & 69.0 & 82.4 & 6.1 & 3.0 & 11.1 \\
\hline Irland & 67.1 & 46.3 & 78.0 & 51.7 & 76.2 & 58.0 & 57.2 & 75.7 & 86.1 & 18.6 & 6.8 & 34.8 \\
\hline Island & 84.4 & 71.6 & 88.2 & 84.8 & 87.4 & 81.2 & 80.5 & 87.7 & 93.3 & 20.1 & 10.2 & 31.2 \\
\hline Italien & 57.5 & 25.5 & 72.2 & 31.4 & 69.7 & 45.3 & 51.6 & 73.5 & 82.3 & 14.7 & 5.3 & 29.2 \\
\hline Japan & 69.3 & 40.9 & 79.0 & 63.9 & 80.4 & 58.1 & 66.7 & 73.6 & 79.2 & 25.8 & 14.2 & 42.3 \\
\hline Kanada & 72.5 & 57.8 & 81.3 & 54.8 & 76.7 & 68.3 & 57.1 & 76.7 & 82.2 & 18.3 & 10.8 & 26.9 \\
\hline Korea & 63.7 & 29.9 & 73.4 & 58.7 & 75.0 & 52.5 & 66.4 & 70.1 & 76.7 & 9.0 & 6.5 & 12.5 \\
\hline Luxemburg & 63.6 & 25.0 & 80.6 & 31.7 & 73.4 & 53.7 & 59.3 & 68.5 & 83.2 & 14.0 & 1.7 & 31.1 \\
\hline Mexiko & 59.6 & 43.7 & 68.7 & 52.5 & 80.2 & 41.5 & 64.8 & 63.8 & 82.2 & 15.1 & 8.1 & 27.6 \\
\hline Neuseeland & 74.6 & 56.9 & 82.0 & 69.7 & 81.5 & 68.0 & 65.1 & 82.0 & 83.9 & 21.7 & 10.2 & 35.3 \\
\hline Niederlande & 71.1 & 61.9 & 80.9 & 44.9 & 77.4 & 64.8 & 59.0 & 77.7 & 85.6 & 35.7 & 15.3 & 60.9 \\
\hline Norwegen & 75.2 & 52.9 & 83.2 & 67.6 & 78.3 & 72.0 & 62.1 & 78.9 & 89.3 & 20.8 & 10.0 & 32.9 \\
\hline Österreich & 68.6 & 53.1 & 82.6 & 31.8 & 75.4 & 62.0 & 52.2 & 73.9 & 82.5 & 16.2 & 4.8 & 29.6 \\
\hline Polen & 53.0 & 20.9 & 69.5 & 29.1 & 59.0 & 47.0 & 37.5 & 61.3 & 82.3 & 11.7 & 7.1 & 17.4 \\
\hline Portugal & 67.5 & 36.1 & 80.8 & 50.5 & 73.4 & 61.7 & 71.9 & 80.3 & 88.0 & 9.8 & 5.9 & 14.4 \\
\hline Schweden & 73.9 & 42.5 & 83.9 & 69.6 & 75.9 & 71.8 & 67.0 & 80.7 & 85.4 & . & $\ldots$ & $\ldots$ \\
\hline Schweiz & 77.2 & 59.9 & 85.1 & 65.0 & 83.9 & 70.4 & 66.1 & 79.8 & 89.7 & 25.1 & 8.0 & 45.8 \\
\hline Slowak. Rep. & 57.7 & 25.6 & 75.3 & 30.4 & 64.6 & 50.9 & 22.0 & 70.3 & 83.6 & 2.6 & 1.4 & 4.1 \\
\hline Spanien & 64.3 & 41.9 & 74.4 & 43.1 & 76.4 & 51.9 & 57.5 & 73.0 & 81.9 & 11.4 & 4.2 & 22.2 \\
\hline Tschech. Rep. & 64.8 & 27.3 & 82.0 & 44.6 & 73.3 & 56.3 & 42.3 & 74.8 & 86.4 & 3.3 & 1.6 & 5.5 \\
\hline Türkei & 45.9 & 31.2 & 54.1 & 30.8 & 68.2 & 23.7 & 50.1 & 61.5 & 75.2 & 5.8 & 3.2 & 13.4 \\
\hline Ungarn & 56.9 & 21.8 & 73.7 & 33.0 & 63.1 & 51.0 & 36.9 & 70.9 & 82.7 & 3.2 & 1.8 & 5.0 \\
\hline Ver. Königreich & 72.6 & 58.1 & 81.1 & 56.8 & 78.6 & 66.8 & 53.0 & 79.4 & 88.8 & 23.6 & 10.0 & 39.3 \\
\hline Ver. Staaten & 71.5 & 53.9 & 79.3 & 60.8 & 77.6 & 65.6 & 56.5 & 72.8 & 82.0 & 12.8 & 7.8 & 18.3 \\
\hline OECD & 66.6 & 42.3 & 78.1 & 49.4 & 74.5 & 58.7 & 56.6 & 74.2 & 83.9 & 15.7 & 7.3 & 26.5 \\
\hline
\end{tabular}

1. 2004 für Deutschland, Niederlande und Schweden; 2004 für den Bildungsabschluss; Teilzeitbeschäftigung: 2004 für Mexiko und 2002 für Island.

Quelle: OECD (2006), OECD-Beschäftigungsausblick, Paris (www.oecd.org/els/employmentoutlook/EmOR006).

Weitere Informationen n OECD (2005), Live Longer, Work Longer, Paris. 


\section{Definition und Messung}

Der hier verwendete Basisindikator für die Arbeitslosigkeit ist die Erwerbslosenquote - d.h. der Anteil der Personen ohne Beschäftigung an der Bevölkerung im erwerbsfähigen Alter (15-64 Jahre). Die in diesem Abschnitt wiedergegebenen Daten stammen aus Arbeitskräfteerhebungen der Mitgliedsländer. Gemäß der ILO-Standarddefinition, die diesen Erhebungen zu Grunde gelegt wird, gelten als erwerbslose Personen diejenigen, die in der Berichtswoche nicht mindestens eine Stunde als abhängige Beschäftigte oder Selbstständige gearbeitet haben, für den Arbeitsmarkt verfügbar sind und in den vier Wochen vor der Erhebung aktive Schritte der Arbeitsuche unternommen haben. Demzufolge werden beispielsweise Personen, die auf Grund physischer Behinderungen nicht arbeiten können, die nicht aktiv nach einer Arbeit suchen, weil sie geringe Hoffnung haben, eine Beschäftigung zu finden, oder die sich in einer Vollzeitbildungsmaßnahme befinden, nicht als erwerbslos betrachtet. Weiter unten werden die Daten nach verschiedenen Kriterien aufgeschlüsselt, d.h. Alter (15-24, 25-54 und 55-64 Jahre), Geschlecht und Bildungsabschluss sowie Dauer der Arbeitslosigkeitsperiode.

Dieser Abschnitt enthält auch Daten über die Inzidenz der Langzeitarbeitslosigkeit unter allen Erwerbslosen. Für die Langzeitarbeitslosen gibt es zwei alternative Definitionen: Personen, die seit mindestens sechs Monaten erwerbslos sind, und Personen mit einer Arbeitslosigkeitsdauer von mindestens zwölf Monaten.

Nachdem die Erwerbslosenquote im OECD-Raum seit 2001 in vier aufeinander folgenden Jahren zugenommen hatte, verringerte sie sich zwischen 2004 und 2005 von 7,3\% auf 7\% (Abb. SS2.1). Die jüngste Entwicklung war in den einzelnen Ländern recht unterschiedlich, mit einem Rückgang der Arbeitslosigkeit in 19 Ländern - darunter Australien, Dänemark, Finnland, Griechenland, Island, Japan, Kanada, Luxemburg, Polen, der Slowakischen Republik, Spanien und den Vereinigten Staaten - und einem fortgesetzten Anstieg in zehn Ländern, vor allem Deutschland, Korea, Norwegen, Mexiko und der Schweiz.

Im Jahr 2005 lagen die Erwerbslosenquoten in elf Ländern bei unter $5 \%$ - darunter Dänemark, Island, Japan, Korea, Mexiko sowie Neuseeland - und in der Türkei, Polen und der Slowakischen Republik bei über 10\% (Tabelle SS2.3). Im Durchschnitt sind junge Menschen, Frauen und gering qualifizierte Arbeitskräfte einem höheren Arbeitslosigkeitsrisiko ausgesetzt als andere. Auch im Ausland geborene Personen sind im Vergleich zur im Inland geborenen Bevölkerung stärker von Arbeitslosigkeit bedroht, wobei es jedoch große Unterschiede zwischen den Ländern gibt. Die Erwerbslosenquote der Frauen ist nur geringfügig höher als die der Männer (durchschnittlich 1,1 Prozentpunkte), allerdings mit sehr viel größeren Differenzen (von bis zu 4 Prozentpunkten oder mehr) in Italien, Spanien und Griechenland. Die Erwerbslosenquote junger Menschen ist wesentlich höher als die von Personen im Haupterwerbsalter (durchschnittlich 9,3 Prozentpunkte) und von älteren Menschen (nahezu 11 Prozentpunkte), während die Erwerbslosenquote älterer Arbeitskräfte generell niedriger ist als die von Personen im Haupterwerbsalter, wobei Deutschland eine Ausnahme bildet. In allen OECD-Ländern besteht für Personen mit niedrigerem Bildungsabschluss eine höhere Wahrscheinlichkeit der Arbeitslosigkeit. Im Durchschnitt der OECD-Länder ist die Erwerbslosenquote bei Personen ohne Sekundarstufe-II-Abschluss um 6 Prozentpunkte höher als bei Personen mit Tertiärabschluss. Besonders hoch sind die Erwerbslosenquoten unter Personen mit niedrigerem Bildungsabschluss in der
Tschechischen Republik, Deutschland, der Slowakischen Republik und Polen.

Im Durchschnitt war nahezu die Hälfte aller Erwerbslosen mindestens sechs Monate und fast ein Drittel mindestens ein Jahr ohne Beschäftigung, mit einer sehr viel höheren Inzidenz ( $50 \%$ oder mehr) in der Slowakischen Republik und Tschechischen Republik, Belgien, Deutschland, Italien und Polen. Bei älteren Menschen ist die Wahrscheinlichkeit der Langzeitarbeitslosigkeit größer als bei Personen anderer Altersgruppen (Abb. SS2.2) auf Grund größerer Schwierigkeiten in Bezug auf die Teilnahme an Weiterbildungs- und Umschulungskursen und möglicherweise auch wegen Altersdiskriminierung. Zwar sind die Arbeitslosigkeitsphasen junger Menschen häufig von kurzer Dauer, doch kann ein wiederholter Wechsel zwischen Arbeitslosigkeit und Kurzzeitjobs zu einer Verschlechterung von Kompetenzen, Fertigkeiten und Motivation führen.

Die Ursachen der Erwerbslosigkeit sind komplexer und vielfältiger Natur. So können Politikmaßnahmen und Institutionen den Effekt nachfrage- und angebotsseitiger Schocks auf die Arbeitslosigkeit mindern, genauso gut aber auch verstärken (OECD, 2006). Arbeitslosigkeit ist nicht nur eine persönliche Tragödie, sie hat auch Auswirkungen auf die Gesellschaft insgesamt, da eine Beschäftigung nicht nur Einkommen und finanzielle Sicherheit mit sich bringt, sondern auch soziale Integration. Die Wahrscheinlichkeit, dass Arbeitslosigkeit zu sozialer Ausgrenzung führt, nimmt mit wachsender Dauer der Arbeitslosigkeitsepisode zu, da die Erwerbslosigkeit möglicherweise mit einem Verlust an Qualifikationen, Gesundheitsproblemen, einem geringen Selbstwertgefühl und sozialer Isolation einhergeht.

Statusindikatoren: Beschäftigung (SS1), Armutspersistenz (EQ7).

Reaktionsindikatoren: Leistungen bei Erwerbslosigkeit (SS6), Öffentliche Sozialausgaben (EQ5). 


\section{SS2.1. Die OECD-weite Enwerbslosenquote verharrt} bei etwa $7 \%$

Erw erbslosenquote für die Altersgruppe 15-64 Jahre, in Prozent der Erwerbsbevölkerung, 1980-2005
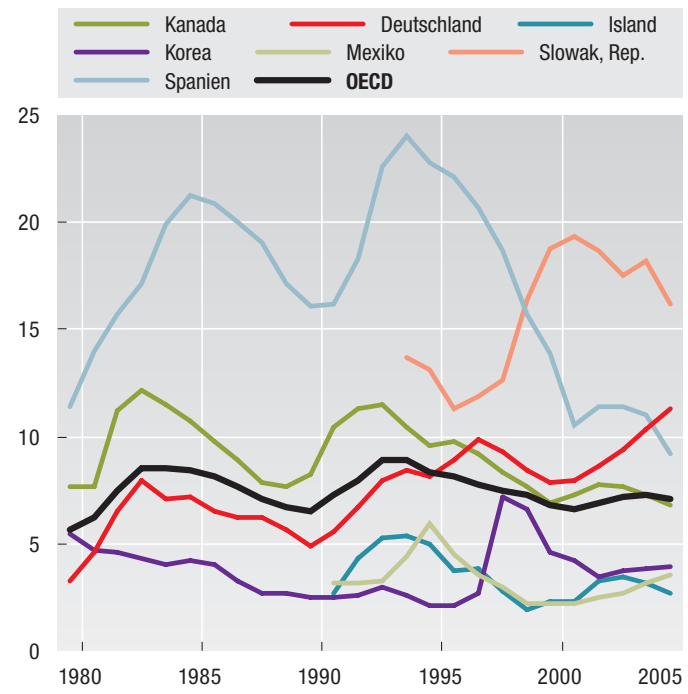

SS2.2. Ältere Personen bleiben länger enwerbslos

Personen mit einer Arbeitslosigkeitsdauer von mindestens 12 Monaten in Prozent aller Erwerbslosen, nach Altersgruppen, $2005^{1}$

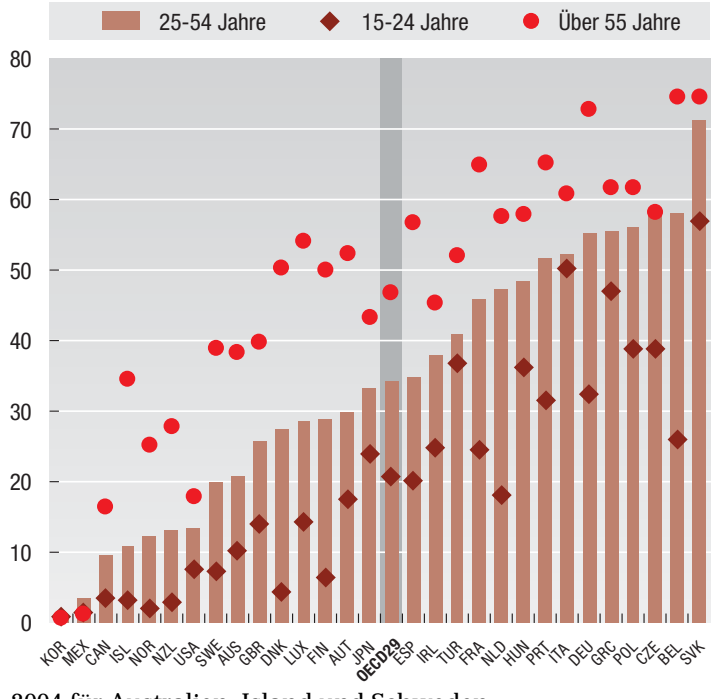

1. 2004 für Australien, Island und Schweden.

SS2.3. Arbeitslosigkeitsindikatoren, $\mathbf{2 0 0 5}^{1}$

\begin{tabular}{|c|c|c|c|c|c|c|c|c|c|c|c|}
\hline & \multirow{3}{*}{$\begin{array}{c}\text { Exwerbslosenquote } \\
\text { für die Bevölkerung } \\
\text { zwischen } 15 \\
\text { und } 64 \text { Jahren }\end{array}$} & \multicolumn{8}{|c|}{ Erwerbslosenquote nach: } & \multirow{2}{*}{\multicolumn{2}{|c|}{$\begin{array}{l}\text { Inzidenz der Langzeit- } \\
\text { arbeitslosigkeit in \% der } \\
\text { Gesamtarbeitslosigkeit }\end{array}$}} \\
\hline & & \multicolumn{3}{|c|}{ Alter } & \multicolumn{2}{|c|}{ Geschlecht } & \multicolumn{3}{|c|}{ Bildungsabschluss } & & \\
\hline & & $15-24$ & $25-54$ & $55-64$ & Männer & Frauen & $\begin{array}{l}\text { Onne Sekundar- } \\
\text { stufe-II-Abschluss }\end{array}$ & $\begin{array}{l}\text { Sekundarstufe- } \\
\text { II-Abschluss }\end{array}$ & $\begin{array}{l}\text { Tertiär- } \\
\text { abschluss }\end{array}$ & $\begin{array}{l}\text { Mindestens } \\
6 \text { Monate }\end{array}$ & $\begin{array}{l}\text { Mindestens } \\
12 \text { Monate }\end{array}$ \\
\hline Australien & 5.2 & 10.8 & 3.9 & 3.2 & 5.0 & 5.3 & 6.2 & 3.9 & 2.8 & 30.2 & 17.7 \\
\hline Belgien & 8.1 & 19.9 & 7.2 & 4.4 & 7.4 & 9.0 & 11.7 & 6.9 & 3.9 & 68.3 & 51.6 \\
\hline Deutschland & 11.3 & 15.2 & 10.4 & 12.7 & 11.5 & 11.0 & 20.5 & 11.2 & 5.5 & 71.0 & 54.0 \\
\hline Finnland & 8.5 & 19.9 & 6.9 & 6.8 & 8.3 & 8.7 & 12.0 & 8.2 & 4.7 & 41.8 & 24.9 \\
\hline Frankreich & 9.9 & 22.8 & 8.7 & 6.8 & 9.0 & 10.9 & 12.1 & 7.6 & 6.2 & 61.2 & 42.5 \\
\hline Griechenland & 9.8 & 25.3 & 8.9 & 3.4 & 5.9 & 15.3 & 8.4 & 9.7 & 6.9 & 72.6 & 53.7 \\
\hline Irland & 4.3 & 8.3 & 3.7 & 2.9 & 4.7 & 3.8 & 6.4 & 3.2 & 2.1 & 52.6 & 34.3 \\
\hline Island & 2.7 & 7.2 & 1.7 & 1.5 & 2.7 & 2.7 & 3.1 & 2.8 & 1.0 & 21.3 & 11.2 \\
\hline Italien & 7.8 & 24.0 & 6.7 & 3.5 & 6.3 & 10.1 & 7.8 & 5.3 & 4.8 & 67.7 & 52.2 \\
\hline Japan & 4.6 & 8.7 & 4.2 & 4.1 & 4.7 & 4.4 & 6.7 & 5.4 & 3.7 & 49.1 & 33.3 \\
\hline Kanada & 6.8 & 12.4 & 5.8 & 5.4 & 7.1 & 6.5 & 9.9 & 6.1 & 4.7 & 17.2 & 9.6 \\
\hline Luxemburg & 4.5 & 13.7 & 3.9 & 2.1 & 3.5 & 5.9 & 5.0 & 3.8 & 3.0 & (51.0) & (26.3) \\
\hline Mexiko & 3.6 & 6.6 & 2.8 & 2.0 & 3.5 & 3.7 & 1.9 & 2.8 & 3.0 & 6.8 & 2.4 \\
\hline Neuseeland & 3.8 & 9.4 & 2.7 & 1.9 & 3.5 & 4.1 & 4.2 & 2.4 & 2.4 & 21.5 & 9.4 \\
\hline Niederlande & 5.2 & 9.6 & 4.4 & 4.5 & 4.9 & 5.6 & 5.7 & 3.9 & 2.8 & 59.9 & 40.1 \\
\hline Norwegen & 4.7 & 12.0 & 4.0 & 1.7 & 4.9 & 4.4 & 3.6 & 3.8 & 2.4 & 25.3 & 9.5 \\
\hline Österreich & 5.2 & 10.3 & 4.4 & 3.6 & 4.9 & 5.5 & 7.8 & 3.8 & 2.9 & 43.2 & 25.3 \\
\hline Polen & 18.0 & 37.8 & 16.0 & 11.2 & 16.9 & 19.4 & 27.8 & 17.4 & 6.2 & 71.6 & 52.2 \\
\hline Portugal & 8.1 & 16.1 & 7.3 & 6.2 & 7.1 & 9.2 & 6.4 & 5.6 & 4.4 & 69.3 & 48.6 \\
\hline Schweden & 7.8 & 22.3 & 6.2 & 4.5 & 7.9 & 7.6 & 6.5 & 5.8 & 4.3 & 37.3 & 18.9 \\
\hline Schweiz & 4.5 & 8.8 & 3.8 & 3.8 & 4.0 & 5.2 & 7.2 & 3.7 & 2.8 & 59.2 & 38.8 \\
\hline Slowak. Rep. & 16.2 & 29.9 & 14.4 & 13.2 & 15.4 & 17.2 & 47.7 & 14.6 & 4.8 & 81.4 & 68.1 \\
\hline Spanien & 9.2 & 19.7 & 8.0 & 6.1 & 7.1 & 12.2 & 11.0 & 9.5 & 7.3 & 47.7 & 32.6 \\
\hline Tschech. Rep. & 8.0 & 19.3 & 7.1 & 5.2 & 6.5 & 9.8 & 23.0 & 6.4 & 2.0 & 72.7 & 53.6 \\
\hline Türkei & 10.5 & 19.3 & 8.7 & 3.4 & 10.5 & 10.6 & 8.1 & 10.1 & 8.2 & 55.6 & 39.6 \\
\hline Ungarn & 7.3 & 19.4 & 6.4 & 3.9 & 7.0 & 7.5 & 10.8 & 5.0 & 1.9 & 63.4 & 46.1 \\
\hline Ver. Königreich & 4.6 & 11.8 & 3.5 & 2.7 & 5.1 & 4.1 & 6.6 & 3.7 & 2.2 & 38.2 & 22.4 \\
\hline Ver. Staaten & 5.1 & 11.3 & 4.1 & 3.3 & 5.1 & 5.2 & 10.5 & 5.6 & 3.3 & 19.6 & 11.8 \\
\hline
\end{tabular}

1. 2004 für Deutschland, Niederlande und Schweden; 2004 für die Daten nach Bildungsabschluss; die Daten in Klammern für Luxemburg basieren auf kleinen Stichproben, bei der Interpretation ist daher Vorsicht geboten.

Quelle: OECD (2006), OECD-Beschäftigungsausblick, Paris (www.oecd.org/els/employmentoutlook/EmO2006).

StatLink 2 http://dx.doi.org/10.1787/043252338503

Weitere Informationen - Bassanini, A. und R. Duval (2006), “Employment Patterns in OECD Countries: Reassessing the Role of Policies and Institutions", OECD Social, Employment and Migration Working Paper, No. 35, Paris. — OECD (2005), Live Longer, Work Longer, Paris. 


\section{Definition und Messung}

In allen OECD-Ländern sind Mütter mit Schwierigkeiten konfrontiert, wenn sie ihre familiären Pflichten mit einer Erwerbstätigkeit in Einklang zu bringen suchen. Um zu veranschaulichen, wie groß die bestehenden Hindernisse sind, werden in diesem Abschnitt Messungen der Erwerbstätigenquoten von Müttern nach der Kinderzahl (ein Kind bzw. zwei oder mehr Kinder) und dem Kinderalter (unter 3, zwischen 3 und 6 sowie zwischen 6 und 14 Jahren) präsentiert und mit den Quoten von kinderlosen Frauen verglichen. Die Kategorie erwerbstätige Frauen umfasst auch Teilzeitbeschäftigte, und die Daten sind nicht in Vollzeitäquivalenten ausgedrückt.

Die Daten zu den Erwerbstätigenquoten sind nationalen Arbeitskräfteerhebungen entnommen und erstrecken sich generell auf das Jahr 2003. Die hier wiedergegebenen Daten beziehen sich auf Frauen, die in einem Beschäftigungsverhältnis stehen (also auch Frauen, die in Mutterschutz oder aus sonstigen Gründen kurzfristig von ihrem Arbeitsplatz abwesend sind) und nicht auf Frauen, die als "erwerbstätig" eingestuft sind (d.h. solche, die angeben, in der Berichtswoche der Erhebung mindestens eine Stunde gearbeitet zu haben). Der Unterschied zwischen diesen beiden Messgrößen der Erwerbstätigenquoten dürfte in Ländern mit Regelungen für lange Elternurlaube, wie z.B. Finnland, Österreich und Schweden, besonders ausgeprägt sein.

Die Erwerbstätigenquoten der Frauen sind in den vergangenen zehn Jahren in nahezu allen Ländern gestiegen: Im Jahr 2003 reichten sie von weniger als 30\% in der Türkei bis zu über $80 \%$ in Island. Jedoch liegen die Erwerbstätigenquoten von Müttern im Allgemeinen deutlich unter denen kinderloser Frauen, vor allem bei Müttern mit zwei oder mehr Kindern (unter 14 Jahren). Im Durchschnitt der OECDLänder beträgt die Differenz bei den Erwerbstätigenquoten etwa 4 Prozentpunkte bei Müttern mit einem Kind (Abb. SS3.1) und 13 Prozentpunkte bei Müttern mit zwei oder mehr Kindern (Abb. SS3.2). Diesbezüglich gibt es große Unterschiede zwischen den Ländern. In sieben Ländern (darunter Dänemark, Portugal und Frankreich) sind die Erwerbstätigenquoten von Müttern mit einem Kind höher als die kinderloser Frauen (Abb. SS3.1), in Schweden wie auch in Kanada bestehen zwischen den Erwerbstätigenquoten dieser beiden Frauengruppen nur geringfügige Unterschiede. Bei Müttern mit zwei oder mehr Kindern hingegen ist die Erwerbstätigenquote nur in Portugal und Schweden mit der kinderloser Frauen vergleichbar (oder höher). Besonders groß sind die Disparitäten bei den Erwerbstätigenquoten in Irland, Neuseeland und der Tschechischen Republik bei Müttern mit nur einem Kind (etwa 15 Prozentpunkte) ebenso wie bei Müttern mit zwei oder mehr Kindern (wo der Abstand über 20 Prozentpunkte beträgt). Letzteres gilt auch für Ungarn und Deutschland.

Die Erwerbstätigenquoten der Mütter hängen auch vom Alter des jüngsten Kindes ab (Tabelle SS3.3). Im Durchschnitt gehen Mütter eher einer Erwerbstätigkeit nach, wenn die Kinder älter werden. In Neuseeland, der Tschechischen Republik und Finnland übersteigen die Erwerbstätigenquoten von Müttern, deren jüngstes Kind zwischen 3 und 5 Jahren ist, die der Mütter mit einem Kind von unter 3 Jahren um 15 Prozentpunkte oder mehr. In den gleichen Ländern übertreffen die Erwerbstätigenquoten von Müttern, deren jüngstes Kind zwischen 6 und 14 Jahre alt ist, die von Müttern mit einem Kind unter 3 Jahren um über 30 Prozentpunkte.

Hohe oder steigende Erwerbstätigenquoten von Müttern werden durch eine Kombination von Politikinstrumen- ten erleichtert, die je nach Land mehr oder weniger ausgeprägt sind: Der Zugang zu erschwinglicher Kinderbetreuung, die Großzügigkeit des Elternurlaubs, Steuerund Transfersysteme, die den Anreiz zur Aufnahme einer Beschäftigung nicht nehmen, sowie die Prävalenz flexibler Arbeitszeitregelungen. Die Großzügigkeit der Elternurlaubsregelungen und der staatlichen Leistungen für Kinderbetreuung erklärt die hohen Erwerbstätigenquoten der Mütter in den nordischen Ländern, während es in den Niederlanden, dem Vereinigten Königreich und Australien vor allem flexible Arbeitszeitregelungen und Teilzeitbeschäftigungen sind, die den Frauen die "Vereinbarkeit von Familie und Beruf" ermöglichen (selbst wenn die Kinderbetreuungskapazitäten in den letzten Jahren in allen drei Ländern rasch ausgebaut worden sind). Starke finanzielle Anreize für die Ausübung einer Erwerbstätigkeit und die verbreitete Nutzung von informellen Betreuungsmöglichkeiten begünstigen hohe Erwerbstätigenquoten von Frauen mit Kindern in den Vereinigten Staaten.

Mehr und mehr sucht die staatliche Politik, beide Elternteile, und insbesondere die Mütter, aus einer Reihe von Gründen, zu einem Verbleib im Erwerbsleben zu bewegen; dazu gehören die Förderung der Geschlechtergleichstellung, eine bessere Nutzung der Arbeitsmarktressourcen und die Bekämpfung der Armut (OECD, 2005). Dieselbe Kombination von Maßnahmen, die sich bei der Erhöhung der Erwerbstätigenquoten der Frauen als effizient erwiesen hat, dürfte auch zu einer Steigerung der Geburtenziffern beitragen. In der Tat verzeichnen OECD-Länder mit höheren Frauenerwerbstätigenquoten auch höhere Geburtenziffern (D'Addio und Mira d'Ercole, 2005).

Statusindikatoren: Beschäftigung (SS1), Kinderbetreuungskosten (SS4), Lohngefälle zwischen Männern und Frauen (EQ3).

Reaktionsindikatoren: Öffentliche Sozialausgaben (EQ5). 
SS3.1. Differenz zwischen den Erwerbstätigenquoten von kinderlosen Frauen und Frauen mit einem Kind, 2003

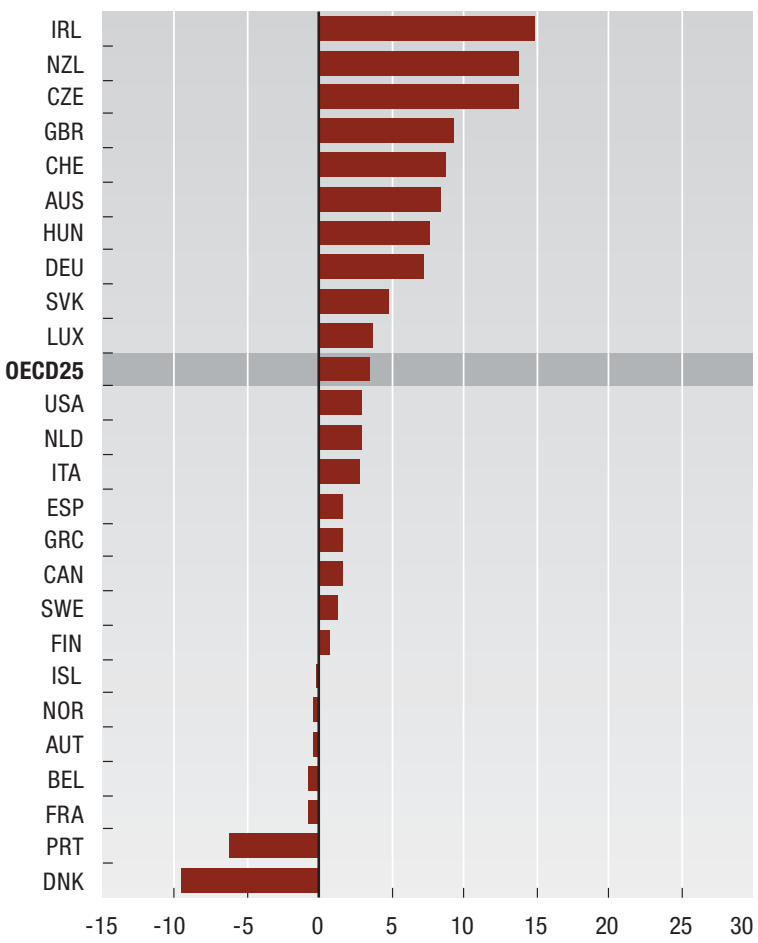

SS3.2. Differenz zwischen den Enwerbstätigenquoten von kinderlosen Frauen und Frauen mit zwei oder mehr Kindern, 2003

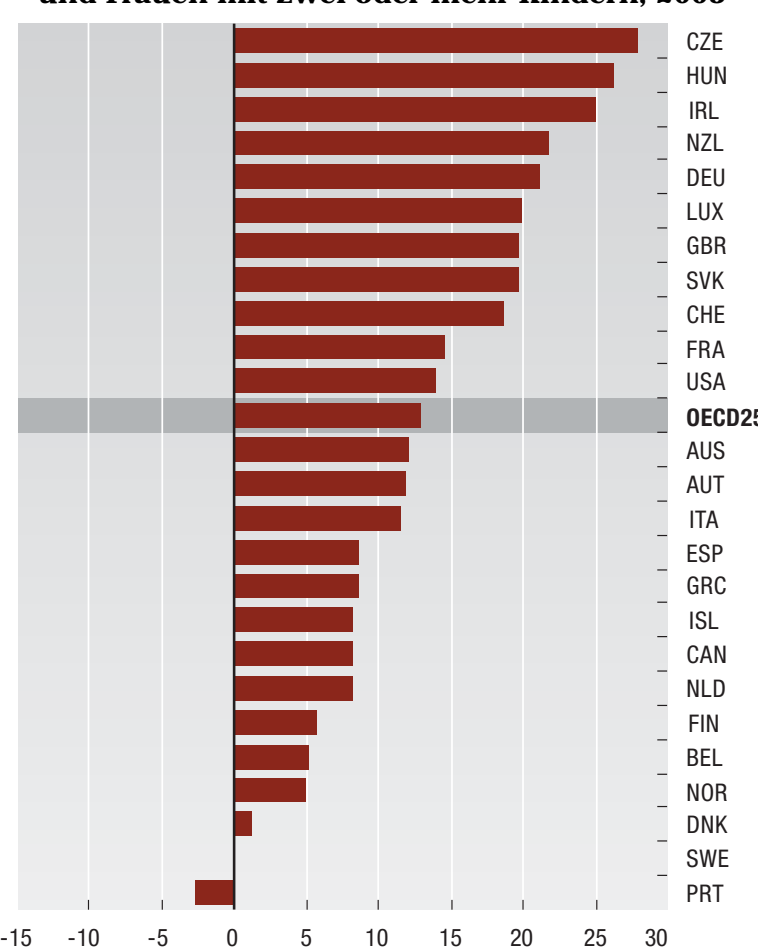

Anmerkung: Eine positive Differenz bedeutet, dass kinderlose Frauen höhere Erwerbstätigenquoten aufweisen als Frauen in den anderen Gruppen. Quelle: Nationale Arbeitskräfteerhebungen aus dem Jahr 2003.

SS3.3. Niedrigere Enwerbstätigenquoten von Müttern, deren jüngstes Kind noch keine drei Jahre alt ist Erwerbstätigenquoten der Mütter nach dem Alter des jüngsten Kindes, $2002^{1}$, in Prozent der Altersgruppe 15-64 Jahre

\begin{tabular}{|c|c|c|c|c|c|c|c|}
\hline & \multicolumn{3}{|c|}{ Alter des jüngsten Kindes } & & \multicolumn{3}{|c|}{ Alter des jüngsten Kindes } \\
\hline & Unter $3^{2}$ & 3-5 & $6-14^{3}$ & & Unter $3^{2}$ & 3-5 & $6-14^{3}$ \\
\hline Belgien & 70.4 & 67.4 & 68.6 & Neuseeland & 43.2 & 58.2 & 74.7 \\
\hline Dänemark & 71.4 & 77.5 & 79.1 & Niederlande & 74.2 & 68.2 & 70.1 \\
\hline Deutschland & 56.0 & 58.1 & 64.3 & Österreich & 80.1 & 70.3 & 69.8 \\
\hline Finnland & 32.2 & 74.7 & 85.3 & Portugal & 75.3 & 81.9 & 76.3 \\
\hline Frankreich & 66.2 & 63.2 & 67.5 & Schweden & 72.9 & 82.5 & 77.4 \\
\hline Giechenland & 47.9 & 50.9 & 53.5 & Schweiz & 58.2 & 64.5 & 77.8 \\
\hline Italien & 54.4 & 51.7 & 49.4 & Tschech. Rep. & 16.8 & 36.5 & 69.2 \\
\hline Kanada & 58.7 & 68.1 & 76.3 & Ver. Königreich & 57.2 & 56.9 & 67.0 \\
\hline \multirow[t]{2}{*}{ Luxemburg } & 70.6 & 63.1 & 58.2 & Ver. Staaten & 56.6 & 60.0 & 69.4 \\
\hline & & & & OECD18 & 59.0 & 64.1 & 69.7 \\
\hline
\end{tabular}

1. 2001 für Dänemark, Irland, Japan, Kanada, Neuseeland und die Vereinigten Staaten.

2. Bei den Angaben sind Mütter in kurzem und langem Mutterschafts- oder Elternurlaub mit berücksichtigt. Eine Ausklammerung dieser Mütter könnte einen signifikanten Effekt auf die gemessenen Beschäftigungsquoten haben; beispielsweise würden die Beschäftigungsquoten für Mütter, deren jüngstes Kind unter 3 Jahre alt ist, deutlich niedriger ausfallen und dann in Österreich 40,1\%, in Finnland 33,8\% und in Schweden $45,1 \%$ betragen.

3. 6- bis 13-Jährige in den Vereinigten Staaten; 6- bis 16-Jährige in Finnland, Kanada, Schweden; 6- bis 17-Jährige in Neuseeland.

Weitere Informationen a D'Addio, A. und M. Mira d'Ercole (2005), “Trends and Determinants of Fertility Rates in OECD Countries: The Role of Policies", OECD Social, Employment and Migration Working Paper, No. 27, Paris. — OECD (2005), Extending Opportunities - How Active Labour Market Policies Can Benefit Us All, Paris. 


\section{Definition und Messung}

Dieser Indikator quantifiziert den Kostenbeitrag der Eltern für die institutionelle Kinderbetreuung. Dieser Posten setzt sich aus einer Vielzahl von Komponenten zusammen, darunter den Gebühren, die die Kinderbetreuungseinrichtungen in Rechnung stellen, sowie den steuerlichen Vergünstigungen und Geldleistungen, die die Eltern für die Betreuung von Kindern erhalten können. Ermittelt wird der Kostenbeitrag durch einen Vergleich des verfügbaren Einkommens nach Abzug der Ausgaben für die Kinderbetreuung einer Familie, die keine institutionelle Kinderbetreuung in Anspruch nimmt, mit dem einer ansonsten gleichgestellten Familie, die dies tut. Die Schätzungen der Kinderbetreuungskosten werden aufgeschlüsselt, um die verschiedenen Politikinstrumente zu identifizieren, die von staatlicher Seite zur Reduzierung der Kosten eingesetzt werden, und unter Berücksichtigung unterschiedlicher Merkmale von Personen und Haushalten präsentiert, wobei vor allem solche Eltern betrachtet werden, deren Entscheidung für eine Erwerbstätigkeit in besonderem Maße durch finanzielle Anreize beeinflusst wird: Alleinerziehende und Zweitverdiener mit kleinen Kindern, die Betreuung benötigen.

Die Berechnungen beziehen sich auf die Kosten einer Vollzeitbetreuung für zwei Kinder im Alter von zwei und drei Jahren in einer klassischen Betreuungseinrichtung. Die Nettobetreuungskosten ergeben sich aus der Differenz zwischen den Gebühren (Bruttobeträge, die den Eltern für einen Monat Vollzeitbetreuung unter Berücksichtigung etwaiger Subventionen für den Anbieter in Rechnung gestellt werden) und allen Arten von Geldleistungen, die die Eltern für Kinderbetreuung erhalten, darunter auch steuerliche Vorteile oder Nachlässe für die Kinderbetreuung. Die Daten beziehen sich auf das Jahr 2001, außer für Kanada (1998), Portugal (2000), Neuseeland, Spanien, Schweden und die Vereinigten Staaten (2002) sowie Belgien, Frankreich und das Vereinigte Königreich (2003). Bei einigen Ländern betreffen die verfügbaren Informationen eine bestimmte Region oder Stadt: Österreich (Wien), Belgien (Wallonien), Kanada (Ontario), Deutschland (Nordrhein-Westfalen), Island (Reykjavík), Schweiz (Zürich), Vereinigte Staaten (Michigan). Nähere Einzelheiten finden sich in den Anhangstabellen A1-A2 in Immervoll und Barber (2005).

Die Kinderbetreuungskosten können ein wichtiges Hindernis für die Aufnahme einer Erwerbstätigkeit darstellen. Im Durchschnitt belaufen sich die aufzubringenden Kosten für die Vollzeitbetreuung von zwei Kindern auf $17 \%$ des Nettohaushaltseinkommens, und zwar sowohl für einen Alleinerziehenden mit dem Verdienst eines Durchschnittsarbeiters als auch für einen Doppelverdienerhaushalt, in dem ein Ehepartner den Durchschnittsverdienst bezieht und der andere auf zwei Drittel des Durchschnittsverdiensts kommt (Abb. SS4.1). Im Ländervergleich gehen die Kostenschätzungen weit auseinander. Am teuersten ist die institutionelle Kinderbetreuung in den englischsprachigen Ländern, Portugal und der Schweiz, wo die von Eltern mit zwei kleinen Kindern zu zahlenden Kosten nicht weniger als $20-34 \%$ des gesamten Familienbudgets ausmachen können. Am anderen Ende des Spektrums befindet sich eine Gruppe größtenteils ost- und nordeuropäischer Länder, in denen die Betreuungskosten für zwei Kinder per saldo etwa $10 \%$ oder weniger des gesamten Nettoeinkommens der Familie beanspruchen.

Kostenerwägungen fallen bei Alleinerziehenden, die ohne Hilfe eines Partners auskommen müssen und deshalb in stärkerem Maße auf externe Kinderbetreuung angewiesen sind, bei der Entscheidung über die Aufnahme einer Erwerbstätigkeit natürlich sehr viel mehr ins Gewicht. Auch wenn öffentliche Zuwendungen für alleinerziehende Eltern die selbst zu tragenden Kosten der Eltern in Kanada, Portugal, Neuseeland, den Vereinigten Staaten und Irland erheblich reduzieren, müssten Alleinerziehende mit zwei Kindern bei Entscheidung für eine Erwerbstätigkeit in der Regel mehr als ein Viertel ihres verfügbaren Einkommens für Kinderbetreuung ausgeben - ein Betrag, den viele von ihnen wahrscheinlich nicht aufbringen könnten. Hier ist die Rangfolge der Länder ganz anders als bei Haushalten mit zwei
Verdienern. Zum Beispiel liegen die Kinderbetreuungskosten für Alleinerziehende im Vereinigten Königreich unter dem Durchschnitt, während sie für Doppelverdienerhaushalte sehr hoch sind, was sich daraus erklärt, dass Alleinerziehende mit niedrigem Einkommen in diesem Land großzügige Nachlässe oder Geldleistungen für die Kinderbetreuung erhalten.

Unterstützungsleistungen für die Kinderbetreuung sind häufig, aber nicht immer auf Niedrigeinkommensfamilien zugeschnitten. In Dänemark, Irland, Island, Korea, Neuseeland, Österreich, der Slowakischen Republik, Ungarn und den Vereinigten Staaten wenden Doppelverdienerhaushalte auf allen in Tabelle SS4.2 aufgeführten Verdienststufen die gleichen Beträge für Kinderbetreuungskosten auf (2. Teil der Tabelle). Generell müssen Familien mit geringerem Bruttoeinkommen somit einen größeren Anteil ihres Nettohaushaltseinkommens für Kinderbetreuung aufbringen als finanziell besser gestellte Familien (1. Teil von Tabelle SS4.2). Der auf Kinderbetreuung entfallende Anteil am Familieneinkommen wird nicht nur durch die Höhe der Kinderbetreuungskosten, sondern auch die Steuerbelastung der Familien bestimmt. So sind beispielsweise in Belgien die Kinderbetreuungskosten in Prozent des Durchschnittsverdiensts niedriger als in Korea, wegen der wesentlich höheren Besteuerung des Familieneinkommens müssen die meisten belgischen Familien aber letztlich einen größeren Teil ihres Nettoeinkommens für die Kinderbetreuung aufwenden als koreanische Familien.

Statusindikatoren: Erwerbstätige Mütter (SS3). Reaktionsindikatoren: Öffentliche Sozialausgaben (EQ5). 


\section{SS4.1 Kinderbetreuungskosten machen einen großen Teil des Nettoeinkommens von Doppelverdiener- und Alleinerzieherhaushalten aus}

Kinderbetreuungskosten einschließlich Transfers und Steuervergünstigungen für einen Doppelverdienerhaushalt mit einem Vollzeitarbeitseinkommen von $167 \%$ und für einen Alleinerzieherhaushalt mit einem Vollzeitarbeitseinkommen von $100 \%$ des Verdiensts eines Durchschnittsarbeiters, beide mit zwei Kindern

Doppelverdienerhaushalt (ein Durchschnittsverdiener, ein Niedriglohnempfänger)

Alleinerzieherhaushalt (Durchschnittsverdiener)

Von den Eltern zu tragende Kinderbetreuungskosten (in \% des Nettohaushaltseinkommens)

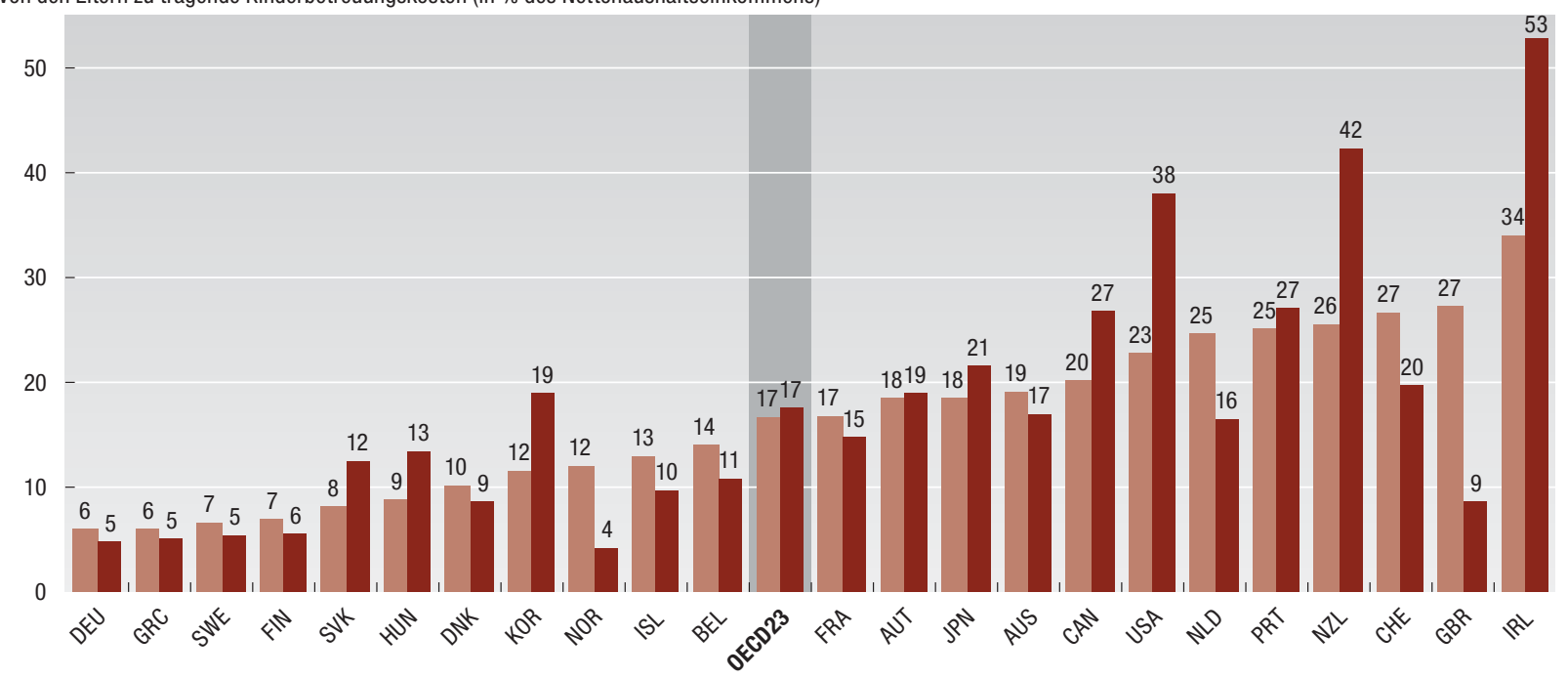

\section{SS4.2 Kinderbetreuungskosten stellen für Niedrigeinkommensfamilien in mehreren OECD-Ländern eine starke Belastung dar}

Kinderbetreuungskosten für Doppelverdienerhaushalte mit zwei Kindern auf drei Verdienststufen, in Prozent des Nettohaushaltseinkommens und des Verdiensts eines Durchschnittsarbeiters

\begin{tabular}{|c|c|c|c|c|c|c|}
\hline & \multicolumn{3}{|c|}{ In \% des Nettohaushaltseinkommens } & \multicolumn{3}{|c|}{ In \% des Bruttoverdiensts eines Durchschnittsarbeiters } \\
\hline & $100-100$ & $100-67$ & $67-67$ & $100-100$ & $100-67$ & $67-67$ \\
\hline Australien & 22 & 19 & 18 & 34 & 26 & 20 \\
\hline Belgien & 13 & 14 & 11 & 16 & 16 & 11 \\
\hline Dänemark & 9 & 10 & 12 & 10 & 10 & 10 \\
\hline Deutschland & 6 & 6 & 5 & 8 & 7 & 5 \\
\hline Finnland & 8 & 7 & 6 & 11 & 9 & 6 \\
\hline Frankreich & 18 & 17 & 15 & 28 & 23 & 17 \\
\hline Griechenland & 6 & 6 & 6 & 10 & 9 & 7 \\
\hline Irland & 29 & 34 & 40 & 51 & 51 & 51 \\
\hline Island & 12 & 13 & 15 & 18 & 18 & 18 \\
\hline Japan & 16 & 18 & 16 & 25 & 25 & 18 \\
\hline Kanada & 18 & 20 & 24 & 28 & 27 & 27 \\
\hline Korea & 10 & 12 & 14 & 18 & 18 & 18 \\
\hline Neuseeland & 21 & 26 & 32 & 34 & 34 & 34 \\
\hline Niederlande & 21 & 25 & 21 & 30 & 30 & 21 \\
\hline Norwegen & 11 & 12 & 13 & 16 & 16 & 14 \\
\hline Österreich & 16 & 18 & 21 & 26 & 26 & 26 \\
\hline Portugal & 21 & 25 & 27 & 38 & 38 & 34 \\
\hline Schweden & 6 & 7 & 6 & 10 & 8 & 7 \\
\hline Schweiz & 29 & 27 & 24 & 48 & 38 & 28 \\
\hline Slowak. Rep. & 7 & 8 & 10 & 12 & 12 & 12 \\
\hline Ungarn & 8 & 9 & 10 & 12 & 12 & 12 \\
\hline Ver. Königreich & 26 & 27 & 22 & 42 & 38 & 26 \\
\hline Ver. Staaten & 19 & 23 & 26 & 34 & 34 & 34 \\
\hline OECD23 & 15 & 17 & 17 & 24 & 23 & 20 \\
\hline
\end{tabular}

Quelle: Immervoll, H. und D. Barber (2005), "Can Parents Afford To Work? Childcare Costs, Tax-Benefit Policies and Work Incentives", OECD Social, Employment and Migration Working Paper, No. 31, Paris (ww w.oecd.org/els/w orkingpapers).

$$
\text { StatLink } 2
$$

http://dx.doi.org/10.1787/082738183216

Weitere Informationen — OECD (2006), Starting Strong, Paris (w w w.oecd.org/edu/earlychildhood). — OECD (2007), Ben efits and Wages - OECD Indicators, Paris (www.oecd.org/els/social/w orkincentives). 


\section{Definition und Messung}

Die in diesem Abschnitt dargelegte Messgröße des Steuer- und Abgabenkeils wird definiert als die Differenz zwischen den dem Arbeitgeber für einen alleinstehenden Durchschnittsarbeiter entstehenden Lohnkosten und der Höhe des verfügbaren Einkommens (Nettolohn) dieses Arbeiters. Bei den hier berücksichtigten Steuern und Abgaben handelt es sich um Einkommensteuern, Arbeitgeber- und Arbeitnehmerpflichtbeiträge zur Sozialversicherung sowie Lohnsummensteuern für die wenigen Länder, in denen solche Steuern existieren. Die Höhe dieser Steuern und Abgaben wird in Prozent der Gesamtarbeitskosten der Unternehmen ausgedrückt (d.h. Bruttolohn plus Arbeitgebersozialabgaben und Lohnsummensteuer). Der "Durchschnittsarbeiter" ist definiert als ein vollzeitbeschäftigter Arbeitnehmer in den Wirtschaftszweigen C-K der Internationalen Wirtschaftssystematik ISIC, Rev. 3.

Dieser Indikator ist abgeleitet aus dem OECD-Bericht Taxing Wages, Ausgabe 2005. Dieser Bericht enthält Daten über die von verschiedenen Arbeitskräftekategorien entrichteten Steuern und Abgaben (Alleinstehende, aber auch verheiratete Paare mit unterschiedlicher Kinderzahl und unterschiedlichem Verdienstniveau), die mit Hilfe der OECD-Steuer- und -Transfermodelle ermittelt wurden. Diese Modelle legen die steuerlichen Bestimmungen jedes Landes zu Grunde und beruhen auf gemeinsamen Typologien und Definitionen der unterschiedlichen Steuerarten. Anzumerken ist, dass ab 2005 eine neue und umfassendere Definition des Durchschnittslohns verwendet wird, was zur Folge hat, dass die hier für die Jahre 2000-2005 dargelegten Werte für den Steuer- und Abgabenkeil auf den Faktor Arbeit nicht mit denen in früheren Ausgaben von Gesellschaft auf einen Blick vergleichbar sind.

Der Steuer- und Abgabenkeil auf den Faktor Arbeit gibt Aufschluss darüber, inwieweit das Steuersystem die Beschäftigung hemmt. Im Durchschnitt der 30 OECD-Länder machte die Steuer- und Abgabenbelastung für einen alleinstehenden Durchschnittsarbeiter 2005 etwa 37\% der Arbeitskosten aus, wobei die Spanne von $50 \%$ oder mehr in Deutschland, Belgien und Ungarn bis zu weniger als $20 \%$ in Korea und Mexiko reichte (Tabelle SS5.1). Im Durchschnitt ist dieser Steuer- und Abgabenkeil seit 2000 um rund $1 / 2$ Prozentpunkt gesunken, mit Rückgängen von über 3 Prozentpunkten in Dänemark, Finnland, Irland und der Slowakischen Republik und Zunahmen um mindestens 2 Prozentpunkte in Island, Japan und der Türkei. Die jeweilige Kombination aus Steuern und Sozialversicherungsabgaben ist ebenfalls unterschiedlich. Die Einkommensteuer machte in Korea und Griechenland 2004 weniger als 5\% der Gesamtarbeitskosten aus, in Dänemark hingegen über $30 \%$ (Abb. SS5.2). Was die Arbeitnehmerbeiträge zur Sozialversicherung betrifft, gibt es zwischen den Ländern eine große Variationsbreite, die sich von null in Australien und Neuseeland bis zu annähernd $20 \%$ in den Niederlanden und Polen erstreckt. Die Arbeitgeberbeiträge zur Sozialversicherung sind die bedeutendste Komponente des Steuer- und Abgabenkeils auf den Faktor Arbeit (sie machen im Durchschnitt etwa $15 \%$ der Gesamtarbeitskosten aus), hier reicht die Palette von nahezu null in Neuseeland und Dänemark über $20 \%$ oder mehr in verschiedenen europäischen Ländern (Slowakische Republik, Griechenland, Spanien, Tschechische Republik, Italien, Österreich, Schweden, Ungarn und Belgien) bis zu fast $30 \%$ in Frankreich.

Die Steuern auf den Faktor Arbeit sind eine wichtige Komponente der Gesamtstaatseinnahmen. In OECD-Ländern mit einem höheren Steuer- und Abgabenkeil auf den Faktor Arbeit ist im Allgemeinen auch die Gesamtsteuerbelastung (gemessen anhand der gesamten Staatseinnahmen im Verhältnis zum BIP, Abb. SS5.3) höher. Es besteht jedoch eine große Variationsbreite. So können Länder mit ähnlichem Niveau der Staatseinnahmen (z.B. Island und
Italien) ganz erhebliche Unterschiede beim Steuer- und Abgabenkeil auf den Faktor Arbeit aufweisen (der im zweiten Land um 16 Prozentpunkte höher ist als im ersten). Dieser Unterschied, der die Bedeutung anderer Steuerarten - indirekte Steuern, Umweltsteuern, Alkohol- und Tabaksteuern - widerspiegelt, kann sich insofern auf die Arbeitsmarktergebnisse auswirken, als Steuern, die nicht direkt auf den Faktor Arbeit erhoben werden, weniger verzerrende Effekte auf Beschäftigungsentscheidungen haben.

Der Steuer- und Abgabenkeil auf den Faktor Arbeit ist eine wichtige Bestimmungsgröße der Arbeitslosigkeit in den OECD-Ländern (OECD, 2006). Bei einer umfassenden Evaluierung der Effekte von Steuern auf den Arbeitsmarkt müssen indessen die Verteilung der Steuerlast sowie die Verwendung der Steuereinnahmen berücksichtigt werden. So können Steuern und Abgaben auf den Faktor Arbeit der Finanzierung von Programmen dienen, die Einkommen an andere Personen umverteilen (eine Steuer im eigentlichen Sinne) oder von Leistungen, auf die der einzelne Arbeitnehmer in einer anderen Lebensphase Anspruch hat (eine Form des Sparens). Schätzungen der Größenordnung der Umverteilungs- und der Rückstellungskomponente bei den Beiträgen zu öffentlichen Rentensystemen (d.h. des Anteils der Arbeitsverdienste, der zur Finanzierung der laufenden Ausgaben der gesetzlichen Rentenversicherung benötigt wird, ohne Budgetzuschüsse oder Aufstockung bzw. Abbau der Rentenmittel) legen den Schluss nahe, dass die Umverteilungsteuer weniger als ein Viertel des Gesamtbetrags ausmacht (Disney, 2004).

Statusindikatoren: Beschäftigung (SS1), Arbeitslosigkeit (SS2).

Reaktionsindikatoren: Leistungen bei Erwerbslosigkeit (SS6), Öffentliche Sozialausgaben (EQ5). 
SS5.1 Weitgehende Stabilität des Steuer- und Abgabenkeils auf den Faktor Arbeit

\begin{tabular}{|c|c|c|c|c|c|c|c|c|c|c|c|c|c|}
\hline & 2000 & 2001 & 2002 & 2003 & 2004 & 2005 & & 2000 & 2001 & 2002 & 2003 & 2004 & 2005 \\
\hline Australien & 30.6 & 27.3 & 27.7 & 28.0 & 28.0 & 28.3 & Niederlande & 39.7 & 37.2 & 37.4 & 37.1 & 38.6 & 38.6 \\
\hline Belgien & 57.1 & 56.7 & 56.3 & 55.7 & 55.4 & 55.4 & Norwegen & 38.6 & 39.2 & 38.6 & 38.1 & 38.1 & 37.3 \\
\hline Dänemark & 44.3 & 43.6 & 42.6 & 42.6 & 41.3 & 41.4 & Österreich & 47.3 & 46.9 & 47.1 & 47.4 & 47.5 & 47.4 \\
\hline Deutschland & 53.9 & 53.0 & 53.6 & 51.5 & 53.3 & 51.8 & Polen & 43.2 & 42.9 & 42.9 & 43.1 & 43.3 & 43.6 \\
\hline Finnland & 47.8 & 46.4 & 45.9 & 45.0 & 44.5 & 44.6 & Portugal & 37.3 & 36.4 & 36.6 & 36.8 & 36.8 & 36.2 \\
\hline Frankreich & 49.6 & 49.8 & 49.8 & 49.8 & 49.8 & 50.1 & Slowak. Rep. & 41.8 & 42.8 & 42.5 & 42.9 & 42.5 & 38.3 \\
\hline Griechenland & 38.4 & 38.1 & 37.7 & 37.7 & 38.3 & 38.8 & Spanien & 38.6 & 38.8 & 39.1 & 38.5 & 38.7 & 39.0 \\
\hline Irland & 28.9 & 25.8 & 24.5 & 24.2 & 26.2 & 25.7 & Schweden & 50.1 & 49.1 & 47.8 & 48.2 & 48.4 & 47.9 \\
\hline Island & 26.1 & 26.9 & 28.4 & 29.2 & 29.4 & 29.0 & Schweiz & 30.0 & 30.1 & 30.1 & 29.7 & 29.4 & 29.5 \\
\hline Italien & 46.4 & 46.0 & 46.0 & 45.0 & 45.4 & 45.4 & Tschech. Rep. & 42.7 & 42.6 & 42.9 & 43.2 & 43.5 & 43.8 \\
\hline Japan & 24.8 & 24.9 & 30.5 & 27.4 & 27.4 & 27.7 & Türkei & 40.4 & 43.6 & 42.5 & 42.2 & 42.8 & 42.7 \\
\hline Kanada & 33.2 & 32.0 & 32.1 & 32.0 & 32.0 & 31.6 & Ungarn & 52.7 & 54.0 & 53.7 & 50.8 & 51.8 & 50.5 \\
\hline Korea & 16.4 & 16.4 & 16.1 & 16.3 & 17.2 & 17.3 & Ver. Königreich & 32.1 & 31.8 & 31.9 & 33.3 & 33.4 & 33.5 \\
\hline Luxemburg & 38.2 & 36.2 & 33.6 & 34.1 & 34.6 & 35.3 & Ver. Staaten & 29.7 & 29.6 & 29.4 & 29.2 & 29.1 & 29.1 \\
\hline Mexiko & 16.8 & 15.9 & 17.5 & 18.1 & 16.2 & 18.2 & & & & & & & \\
\hline Neuseeland & 19.4 & 19.4 & 19.5 & 19.7 & 20.0 & 20.5 & OECD & 37.9 & 37.5 & 37.5 & 37.2 & 37.4 & 37.3 \\
\hline
\end{tabular}

Anmerkung: Der Steuer- und Abgabenkeil auf den Faktor Arbeit ist definiert als die Summe aus Einkommensteuern und Arbeitnehmer- sowie Arbeitgeberpflichtbeiträgen zur Sozialversicherung für eine alleinstehende Person mit dem Verdienst eines Durchschnittsarbeiters. Dieser Steuer- und Abgabenkeil wird in Prozent der Arbeitskosten ausgedrückt.

\section{SS5.2 Große Unterschiede zwischen den Ländern bei der Zusammensetzung des Steuer- und Abgabenkeils auf den Faktor Arbeit}

Einkommensteuern, Arbeitnehmer- und Arbeitgeberbeiträge zur Sozialversicherung, in Prozent der Arbeitskosten,

für eine alleinstehende Person mit Durchschnittsverdienst, 2005

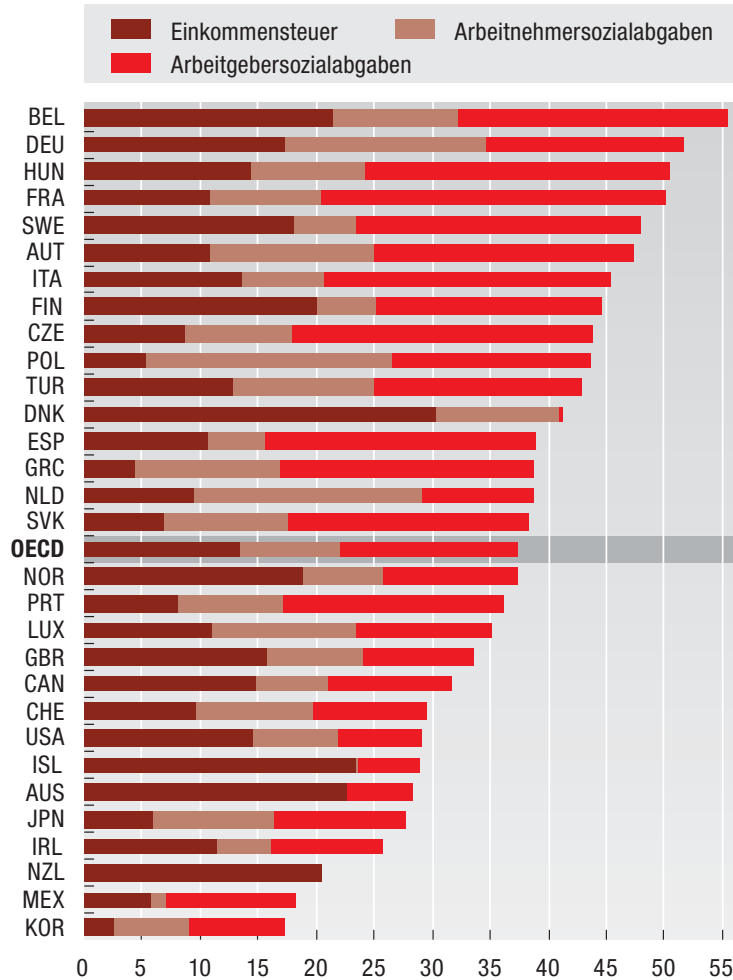

Anmerkung: Die Länder sind in absteigender Reihenfolge nach dem Steuer- und Abgabenkeil von 2005 angeordnet.
SS5.3 Länder mit ähnlichem Steuerund Abgabenkeil auf den Faktor Arbeit können eine ganz unterschiedliche Steuerbelastung haben

Steuer- und A bgabenkeil auf den Faktor Arbeit,

in Prozent der Arbeitskosten sowie Gesamtstaatseinnahmen in Prozent des BIP, letztes verfügbares Jahr

Gesamtstaatseinnahmen, in \% des BIP, 2004

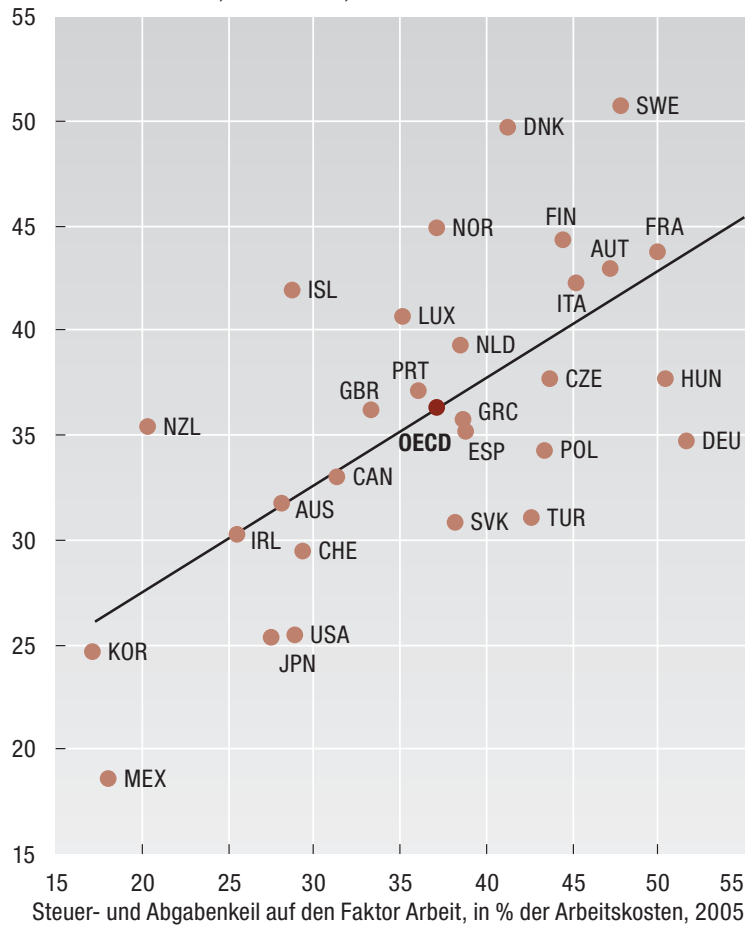

Anmerkung: Der Steuer- und Abgabenkeil auf den Faktor Arbeit bezieht sich auf eine alleinstehende Person mit dem Verdienst eines Durchschnittsarbeiters.

Quelle: OECD (2006), Taxing Wages 2004-2005, Paris (www.oecd.org/ctp/taxingwages) und OECD (2006), Revenue Statistics 1965-2004, Paris (www.oecd.org/ctp/statistics).

StatLink 2 http://dx.doi.org/10.1787/725757462120

Weitere Informationen — Disney, R. (2004), "Are Contributions to Public Pension Programmes a Tax on Employment?", Economic Policy, Juli. @ OECD (2006), "Neubewertung der Rolle von Politiken und Institutionen für die Arbeitsmarktergebnisse: eine quantitative Analyse", Kapitel 7 in OECD-Beschäftigungsausblick, Paris. 


\section{Definition und Messung}

Die Leistungen bei Erwerbslosigkeit werden gemessen als Differenz zwischen dem Nettoeinkommen eines ErwerbsIosen und eines Erwerbstätigen. Der hier dargelegte Hauptindikator ist die Nettoersatzquote, definiert als Verhältnis zwischen dem Nettohaushaltseinkommen bei Erwerbslosigkeit und dem früheren Einkommen bei Erwerbstätigkeit des Familienvorstands. Eine Betrachtung der effektiven Grenzsteuersätze liefert ähnliche Informationen in anderer Form, denn diese Sätze geben Aufschluss über finanzielle Konsequenzen der Aufnahme oder Ausweitung einer Erwerbstätigkeit (d.h. sie messen den prozentualen Anteil der zusätzlichen Arbeitsverdienste, der durch eine Kombination von reduzierten Leistungen und höheren Einkommensteuern "weggesteuert" wird). Der hier dargelegte Indikator der effektiven Steuersätze bezieht sich auf Personen, die zum Zeitpunkt der Wiederaufnahme eines Beschäftigungsverhältnisses auf unterschiedlichem Verdienstniveau weniger als 60 Monate erwerbslos waren.

Diese Schätzungen der Lohnersatzquoten bei Erwerbslosigkeit und der effektiven Steuersätze basieren auf den Steuer- und Transfermodellen der OECD für die einzelnen Länder, bei denen Personen in einer Reihe von "typischen" Situationen betrachtet werden. Folgende Familientypen werden untersucht: Ein- und Zweipersonenhaushalte mit und ohne Kinder (zwei Kinder im Alter von 4 und 6 Jahren) unter der Annahme, dass der Ehepartner weder erwerbstätig ist noch Arbeitslosengeldleistungen bezieht, und ohne Berücksichtigung von Kindergeldleistungen und Kinderbetreuungskosten. Die Ersatzquoten bei Erwerbslosigkeit können je nach Dauer des Leistungsbezugs unterschiedlich sein. Anhand des Durchschnitts dieser Ersatzquoten für die gesamte Dauer der Arbeitslosigkeit und für unterschiedliche Familientypen wird ein Gesamtindikator berechnet: Diese synthetische Messgröße ist ein einfacher Durchschnitt der Nettoersatzquoten, zu dessen Berechnung jeder Leistungsmonat in einem Fünfjahreszeitraum für vier Familientypen und zwei frühere Verdienstniveaus (100\% und $66,7 \%$ des Verdiensts eines Durchschnittsarbeiters) zu gleichen Anteilen gewichtet wird. Es werden separate Schätzungen für Personen mit und ohne Anspruch auf zusätzliche Sozialhilfeleistungen aufgezeigt. Ab 2005 basieren die hier dargelegten Indikatoren auf einer neuen und umfassenderen Definition des "Durchschnittsarbeiters"; infolgedessen weichen die in dieser Ausgabe wiedergegebenen Indikatoren von denen früherer Ausgaben von Gesellschaft auf einen Blick ab.

Die Festlegung des "richtigen" Leistungsniveaus für erwerbslose Personen stellt die Regierungen vor ein Dilemma: Ein sehr niedriges Leistungsniveau kann die Empfänger in eine wirkliche Notlage bringen und es für Arbeitsuchende schwierig machen, der Suche nach einem geeigneten und dauerhaften Arbeitsplatz die notwendige Zeit zu widmen; umgekehrt bieten sehr großzügig bemessene Leistungen dem Einzelnen u.U. nur wenig Anreize für die Arbeitsuche. Die Arbeitslosenunterstützungssysteme in den OECD-Ländern weisen große Unterschiede auf. In den meisten Ländern wird das zeitlich befristete Arbeitslosengeld nach Erlöschen des Anspruchs auf Arbeitslosenversicherungsleistungen durch zusätzliche Sozialhilfeleistungen ergänzt; diese zusätzlichen Sozialhilfeleistungen, die bei langen Arbeitslosigkeitsperioden stärker ins Gewicht fallen, können in einigen Ländern auch in den Anfangsphasen der Arbeitslosigkeit als Ergänzung zum Familieneinkommen gezahlt werden, obwohl dies nicht die Regel ist (die Einkünfte der Betreffenden liegen während dieses Zeitraums häufig über den geltenden Schwellenwerten). Andere Länder (wie z.B. Australien und Neuseeland) bieten Arbeitslosen Einkommenserhalt über eine zeitlich unbefristete Sozialhilfeleistung bei Arbeitslosigkeit. Im Durchschnitt der OECD-Länder lag die synthetische Messgröße der bei Erwerbslosigkeit zur Anwendung kommenden Ersatzquoten 2004 bei $57 \%$, wenn davon ausgegangen wird, dass die arbeitslose Person während der gesamten Arbeitslosigkeitsperiode auf alle existierenden Arten von Sozialhilfeleistungen Anspruch hat, und bei $41 \%$, wenn nur die Arbeitslosenleistungen (Arbeitslosengeld und Arbeitslosenhilfe) berücksichtigt werden (Abb. SS6.1). Der synthetische Indikator der Lohnersatzquoten bei Erwerbslosigkeit unter Berücksichtigung aller zusätzlichen Arten von Sozialhilfeleistungen ist am höchsten in Dänemark und der Schweiz (80\%) und übersteigt 70\% in Finnland, Deutschland, Island, Luxemburg, Niederlande und Schweden; während die niedrigsten Werte (30\% oder weniger) in Griechenland, Ungarn, Italien und den Vereinigten Staaten verzeichnet werden.
In einigen und insbesondere mehreren kontinentaleuropäischen Ländern kann die Aufnahme einer Beschäftigung mit großen, ja schwerwiegenden finanziellen Nachteilen verbunden sein (bei effektiven Steuersätzen von über 100\%), vor allem bei Arbeitsverdiensten, die sich auf ein bis zwei Drittel des Durchschnittsverdiensts belaufen (Abb. SS6.2). Die negativen finanziellen Anreize für die Aufnahme einer Beschäftigung können durch berufsbezogene Ausgaben (z.B. Fahrt- oder Kinderbetreuungskosten) und auf lokaler Ebene gebotene Leistungen (z.B. vergünstigte Tarife für Arbeitslose im Regionalverkehr) noch verstärkt werden. In mehreren Ländern schwanken die effektiven Steuersätze auch je nach Familienstruktur. So sind sie in den Vereinigten Staaten, aber auch in Belgien, der Slowakischen Republik und bei geringerem Verdienstniveau in Polen und dem Vereinigten Königreich für kinderlose Personen wesentlich höher als für Einverdienerhaushalte mit Kindern. Umgekehrt sind die durchschnittlichen effektiven Steuersätze in Australien, Österreich, Finnland, Irland und der Schweiz für Paare mit Kindern höher als für Alleinstehende.

Die Leistungen bei Arbeitslosigkeit stellen wichtige Bestimmungsfaktoren für die finanziellen Anreize einzelner Personen zur Aufnahme einer Erwerbstätigkeit dar, daneben fallen aber noch andere Faktoren ins Gewicht. Hierzu zählen Überprüfungen der Arbeitsbereitschaft, die nach den Arbeitslosengeld- und Sozialhilferegelungen vorgeschrieben sind, das mit Nichterwerbstätigkeit verbundene Stigma wie auch langfristige Überlegungen hinsichtlich Verdienst- und Karriereaussichten, die Arbeitslose dazu bewegen können, ein Stellenangebot anzunehmen, selbst wenn dies kurzfristig eine Reduzierung des Familieneinkommens bedeutet.

Statusindikatoren: Arbeitslosigkeit (SS2), Armutspersistenz (EQ7).

Reaktionsindikatoren: Öffentliche Sozialausgaben (EQ5), Gesamtsozialausgaben (EQ6). 


\section{SS6.1 Arbeitslosenleistungen ersetzen im Durchschnitt $\mathbf{4 0} \%$ des zuvor bezogenen Verdiensts}

Durchschnittliche Nettoersatzquoten in einem Zeitraum von 60 Monaten der Erwerbslosigkeit im Jahr 2004, für vier Familientypen und zwei Verdienstniveaus, in Prozent

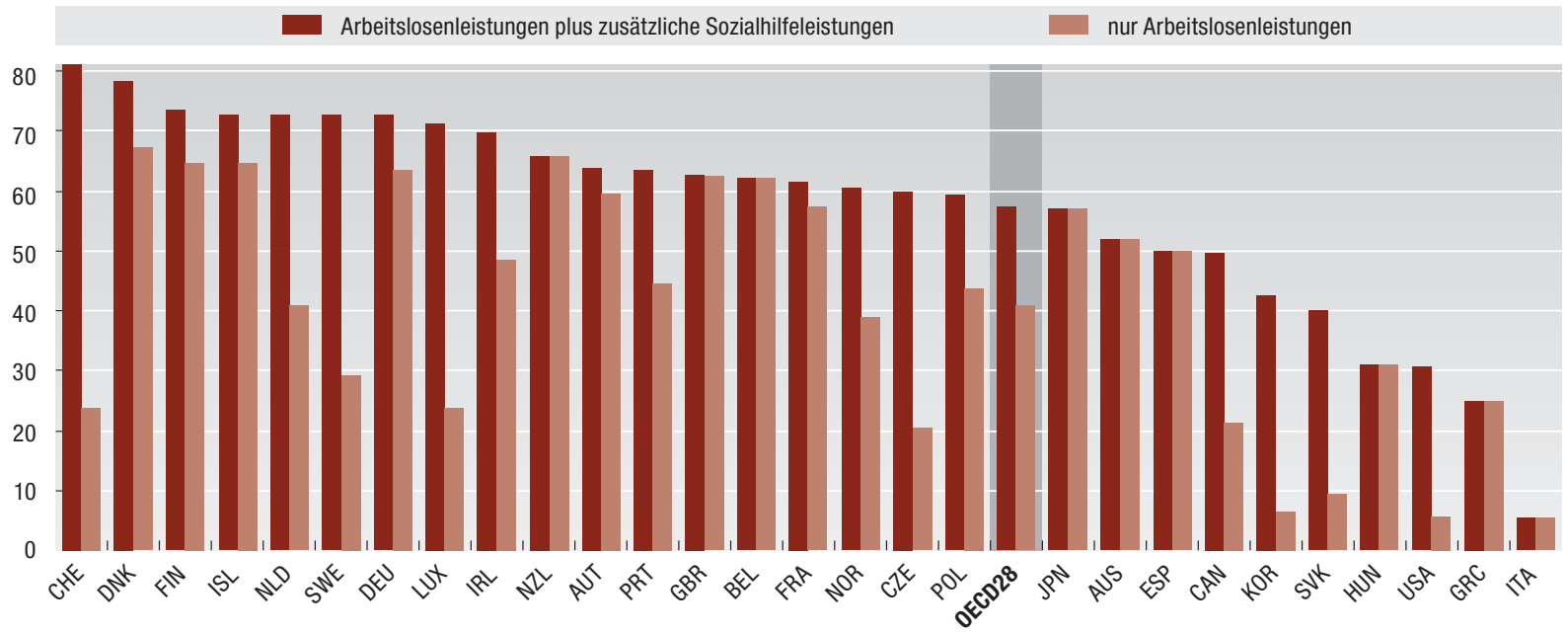

\section{SS6.2 Die Aufnahme einer Enwerbstätigkeit kann in einigen Ländern mit großen finanziellen Nachteilen verbunden sein}

Durchschnittliche effektive Steuersätze (AETR) für Kurzzeitarbeitslose bei Wiederaufnahme einer Beschäftigung, 2004, in Prozent
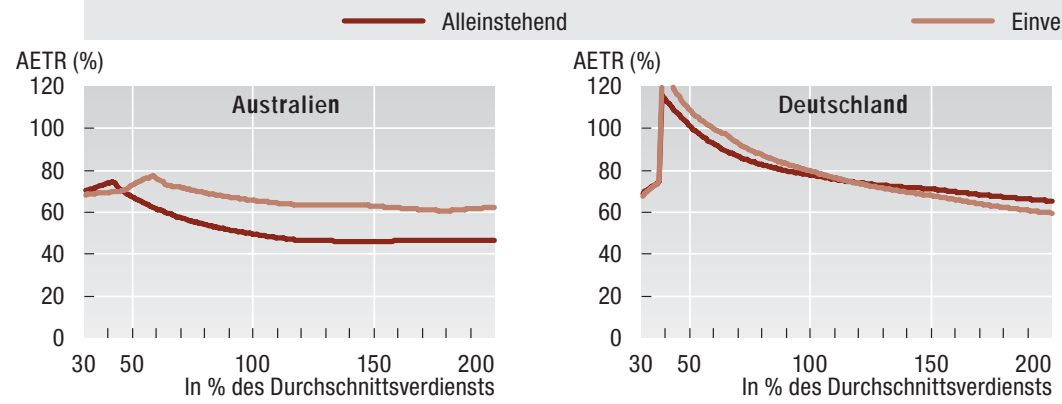

Einverdienerhaushalt mit zwei Kindern
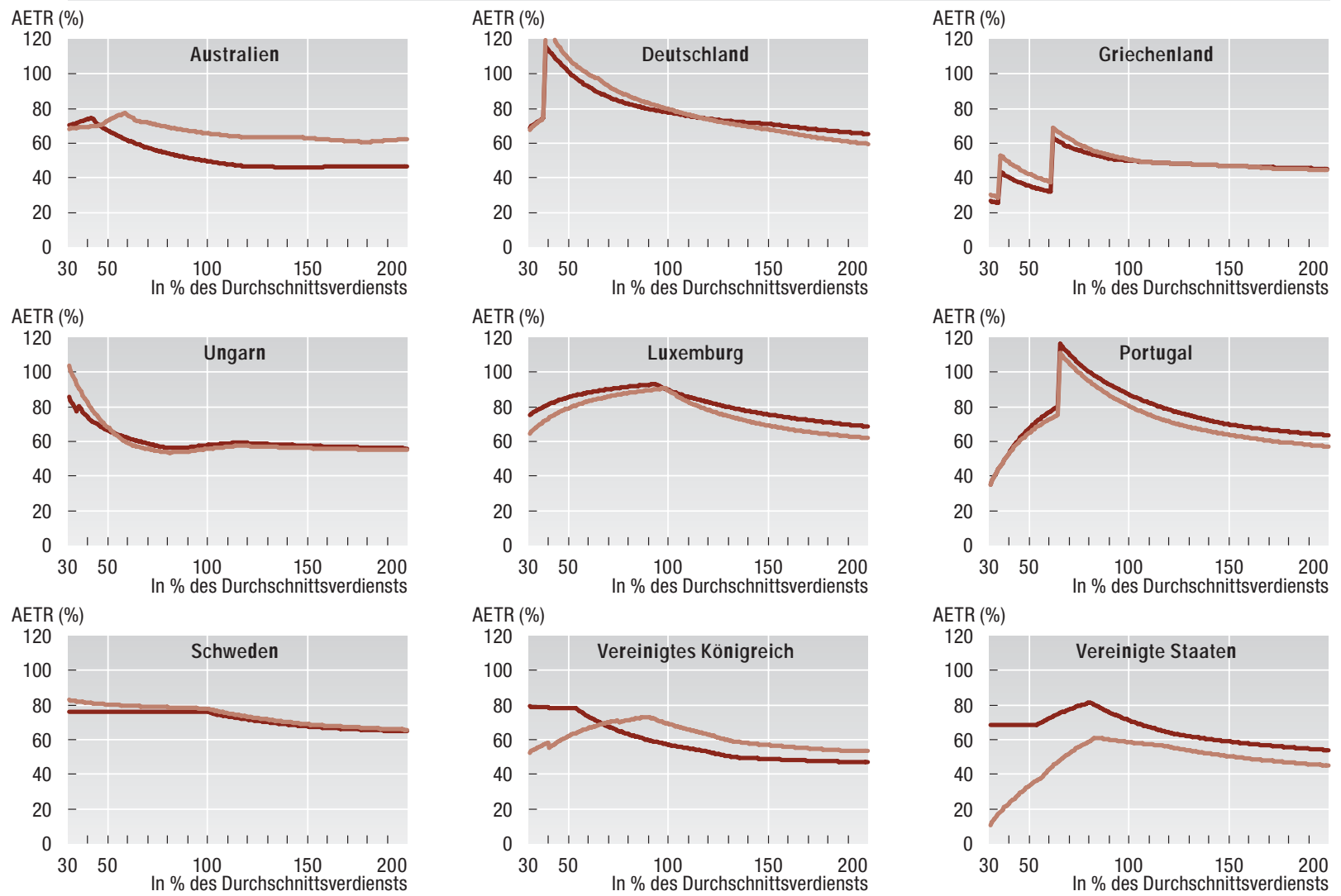

Quelle: OECD (2007), Benefits and Wages, erscheint demnächst, Paris (www.oecd.org/els/social/w orkincentives).

StatLink $2 \quad$ http://dx.doi.org/10.1787/176515702117

Weitere Informationen $\square$ Immervoll, H. (2004), “Average and Marginal Effective Tax Rates Facing Workers in the EU. A microlevel analysis of levels, distributions and driving factors", OECD Social, Employment and Migration Working Paper, No. 19, Paris. Carone, G., H. Immervoll, D. Paturot und A. Salomäki (2004), "Indicators of Unemployment and Low-wage Traps", OECD Social, Employment and Migration Working Paper, No. 18, Paris. 


\section{Definition und Messung}

Die Schülerleistungen lassen sich anhand der Ergebnisse der von der OECD getragenen Internationalen Schulleistungsstudie PISA beurteilen, das bisher umfassendste und weitreichendste internationale Projekt zur Messung der Kenntnisse und Fähigkeiten von Schülerinnen und Schülern gegen Ende ihrer Pflichtschulzeit. Mehr als eine viertel Million 15-jährige Schülerinnen und Schüler in 41 Ländern nahmen am PISA-Testzyklus 2003 teil. Die Tests wurden unter unabhängiger Aufsicht durchgeführt, um die Kompetenzen der Schülerinnen und Schüler in verschiedenen Bereichen zu evaluieren und die Vergleichbarkeit zwischen den Ländern zu gewährleisten. In der PISA-Erhebungsrunde 2003 entfielen 31/2 Stunden Testzeit auf den Bereich Mathematik und jeweils 1 1/2 Stunden auf die Bereiche Lesekompetenz, Naturwissenschaften und Problemlösung. Die PISA-Tests sind nicht an bestimmte nationale Lehrpläne gekoppelt; vielmehr wird von den Schülerinnen und Schülern verlangt, in der Schule erworbenes Wissen auf Situationen anzuwenden, denen sie in der realen Welt begegnen könnten, wie die Planung einer Reiseroute, die Interpretation der Gebrauchsanleitung für ein elektrisches Gerät oder das Auffinden von Informationen in einer graphischen Darstellung. Alle Ergebnisse sind standardisiert, so dass in den OECDLändern die mittlere Punktzahl für jeden Bereich bei 500 liegt. In den Bereichen Lesekompetenz und Naturwissenschaften sind Ergebnisvergleiche zwischen PISA 2003 und PISA 2000 möglich, obgleich Unterschiede zwischen den Erhebungen mit Vorsicht interpretiert werden sollten.

Neben den mittleren Punktzahlen, die die Schüler in jedem Land in den drei Grundbildungsbereichen (Mathematik, Lesekompetenz und Naturwissenschaften) erzielten, präsentiert dieser Abschnitt eine Messgröße der Ungleichheit bei den Testergebnissen in Mathematik, definiert als Verhältnis zwischen der durchschnittlichen Punktzahl der Schülerinnen und Schüler im obersten Quartil und im untersten Quartil der Leistungsskala.

Dafür Sorge zu tragen, dass Kinder eine gute Bildung erhalten, ist in allen OECD-Ländern eine Politikpriorität. Mit der Zunahme der Hochschulbesuchsquoten hat sich die Aufmerksamkeit der Politikverantwortlichen nach und nach auf die Beurteilung der in der Schule erworbenen Kompetenzen verlagert. Wie aus Abbildung SS7.1 hervorgeht, bestehen zwischen den Ländern große Unterschiede beim Leistungsniveau, das die Schülerinnen und Schüler gegen Ende der Pflichtschulzeit aufweisen. 2003 überstiegen die Durchschnittsleistungen der drei bestplatzierten OECDLänder auf der Gesamtskala Mathematik (Finnland, Korea und Niederlande) die entsprechenden Leistungen der drei am unteren Ende der Skala angesiedelten Länder (Mexiko, Türkei und Griechenland) um etwa 120 Punkte, was in Schuljahren ausgedrückt einer Differenz von nahezu drei Jahren entspricht. In den Bereichen Lesekompetenz und Naturwissenschaften sind die Unterschiede bei den Schülerleistungen ähnlich groß. Generell schneiden Länder, die in einem Bereich ganz oben auf der Skala stehen, auch in anderen Bereichen gut $a b$, wie eine Korrelation von über 0,80 zwischen Mathematik und Lesekompetenz bzw. Mathematik und Naturwissenschaften in der Rangfolge der Länder verdeutlicht.

Im Ländervergleich bestehende Unterschiede bei den durchschnittlichen Schülerleistungen erklären sich hauptsächlich durch Disparitäten bei den leistungsschwachen Schülern. In Mathematik sind die Länderunterschiede bei den Schülerleistungen im untersten Quartil der Leistungsskala um rd. 20\% höher als im obersten Quartil und um fast $60 \%$ bei einem Vergleich des unteren und oberen Dezils aller Schülerinnen und Schüler. Daher weisen OECD-Länder mit einer sehr unausgewogenen Verteilung der Testergebnisse der Schüler in Mathematik auch niedrige durchschnittliche Punktwerte auf (Abb. SS7.2).

Diese großen Unterschiede zwischen den Ländern bei den Lernergebnissen der Schülerinnen und Schüler hängen
z.T. mit den Merkmalen des Bildungssystems der einzelnen Länder zusammen. Zwischen den Ausgaben pro Schüler im Alter von 6-15 Jahren und den Schülerleistungen besteht nur ein schwach positiver Zusammenhang. Mehr Belege gibt es indessen dafür, dass die Ungleichheiten bei den Lernergebnissen umso größer und die Durchschnittsleistungen umso niedriger sind, je früher eine Verteilung auf verschiedene Schultypen erfolgt.

Diese Unterschiede bei den Schülerleistungen können langfristige Auswirkungen für die jungen Menschen bei ihrem Übergang ins Erwachsenenleben wie auch für die Gesamtgesellschaft haben. Schwache Lernergebnisse am Ende der Pflichtschulzeit können zu einer höheren Wahrscheinlichkeit des Schulabbruchs vor Abschluss der Sekundarstufe führen, schlechteren Verdienst- und Karriereaussichten beim Eintritt in den Arbeitsmarkt, geringeren Chancen auf einen betrieblichen Ausbildungsplatz und im extremsten Fall einer größeren Wahrscheinlichkeit, im Erwachsenenleben von Sozialhilfe abhängig zu sein. Bildung kann den Aufstieg aus sozialer Benachteilung ermöglichen, sie ist aber auch ein Motor der sozialen Selektion (Machin, 2006). In dem Maße, wie die Sozialpolitik der Arbeitsmarktintegration als dem wichtigsten Instrument zur Bekämpfung von Armut und sozialer Ausgrenzung zunehmende Bedeutung beimisst, müssen die Politikverantwortlichen den Lernergebnissen der Schülerinnen und Schüler am unteren Ende der Leistungsskala größere Aufmerksamkeit widmen.

Statusindikatoren: Beschäftigung (SS1), Verdienstungleichheit (EQ2), Generationenmobilität (EQ4).

Reaktionsindikatoren: Öffentliche Sozialausgaben (EQ5). 


\section{SS7.1 Große Unterschiede bei den Schülerleistungen in den OECD-Ländern}

Mittelwerte auf den Skalen Mathematik, Lesekompetenz und Naturwissenschaften, PISA 2003 und 2000

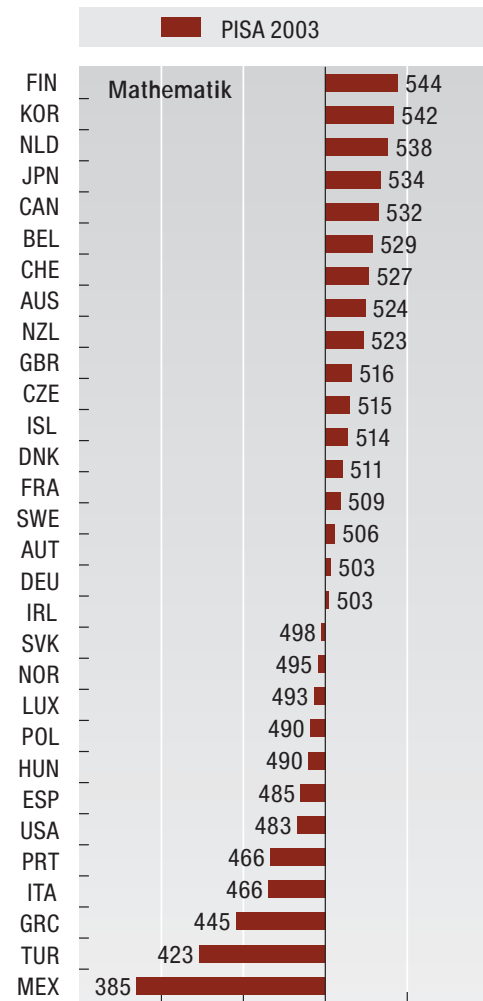

$\begin{array}{llllll}350 & 400 & 450 & 500 & 550 & 600\end{array}$
PISA 2003

PISA 2000

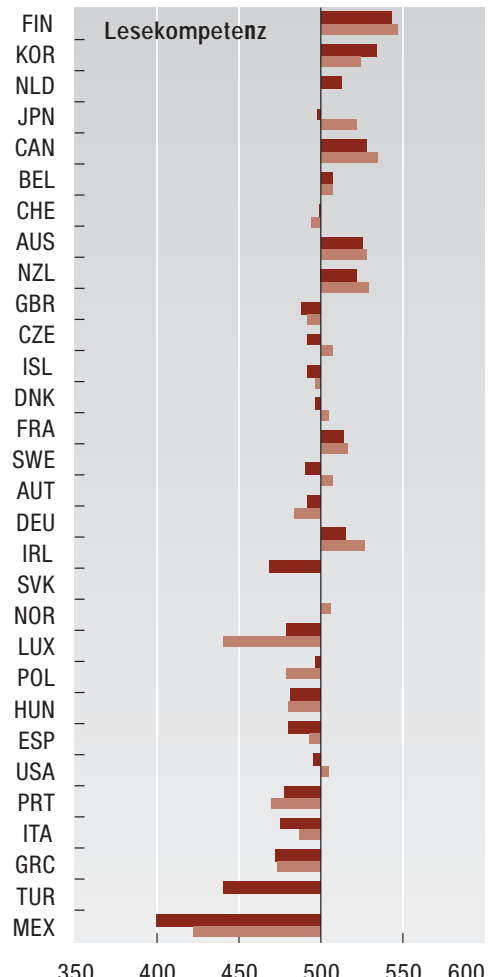

PISA $2003 \quad$ PISA 2000

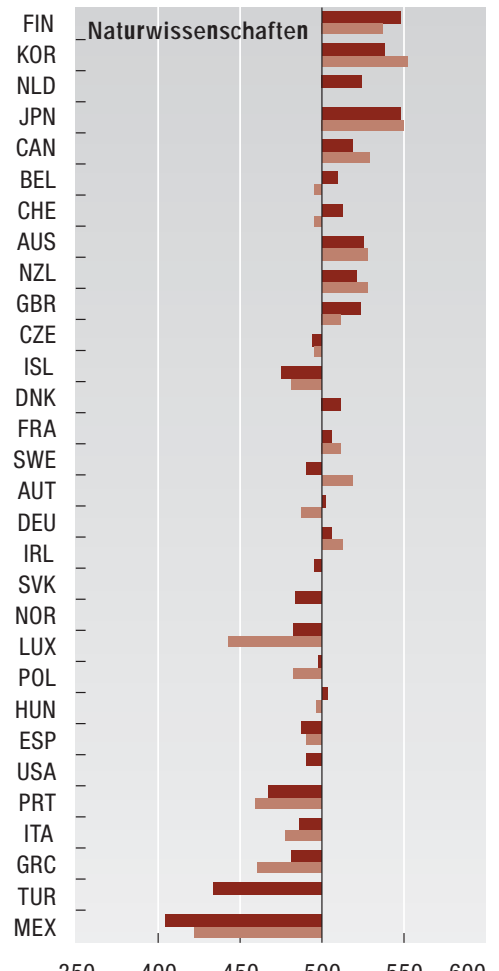

Anmerkung: Die Länder sind von oben nach unten in absteigender Reihenfolge der Schülerleistungen in Mathematik angeordnet. Die angegebenen Werte beziehen sich auf die Differenz des Mittelwerts jeden Landes gegenüber dem OECD-Durchschnitt (500).

\section{SS7.2 Höheres Leistungsniveau der Schüler in Ländern mit geringerer Ungleichheit bei den Testergebnissen der Schüler}

Durchschnittsleistungen in Mathematik und Ungleichheit bei den Schülerpunktzahlen in Mathematik, 2003

Durchschnittsleistungen in Mathematik

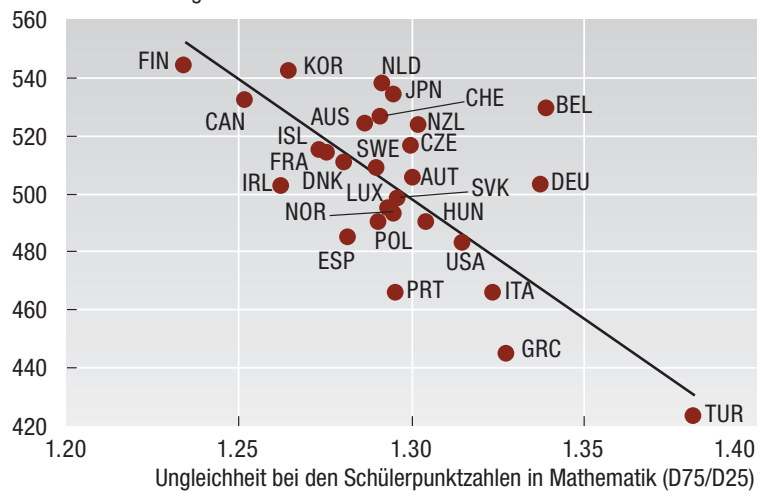

SS7.3 Die durchschnittlichen Schülerleistungen verbessern sich mit höheren Ausgaben je Schüler nur geringfügig

Durchschnittsleistungen in Mathematik und Ausgaben für Bildungseinrichtungen (je Schüler bis 15 Jahre), $2003^{1}$ Durchschnittsleistungen in Mathematik

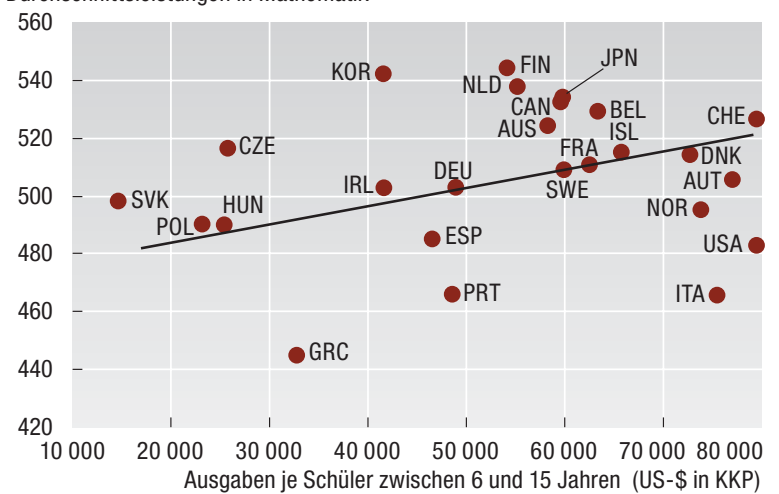

1. Die tatsächlichen Ausgaben je Schüler im Jahr 2002 werden ermittelt durch Multiplikation der öffentlichen und privaten Ausgaben je Schüler auf jeder Bildungsstufe mit der theoretischen Verweildauer auf dieser Stufe, bis zum Alter von 15 Jahren. Quelle: OECD (2004), Lernen für die Welt von morgen - Erste Ergebnisse von PISA 2003, Paris (ww w.pisa.oecd.org).

S tatLink 2 http://dx.doi.org/10.1787/787232841255

Weitere Informationen $\square$ Machin, S. (2006), "Social Advantage and Education Experiences", OECD Social, Employment and Migration Working Paper, No. 32, Paris. 



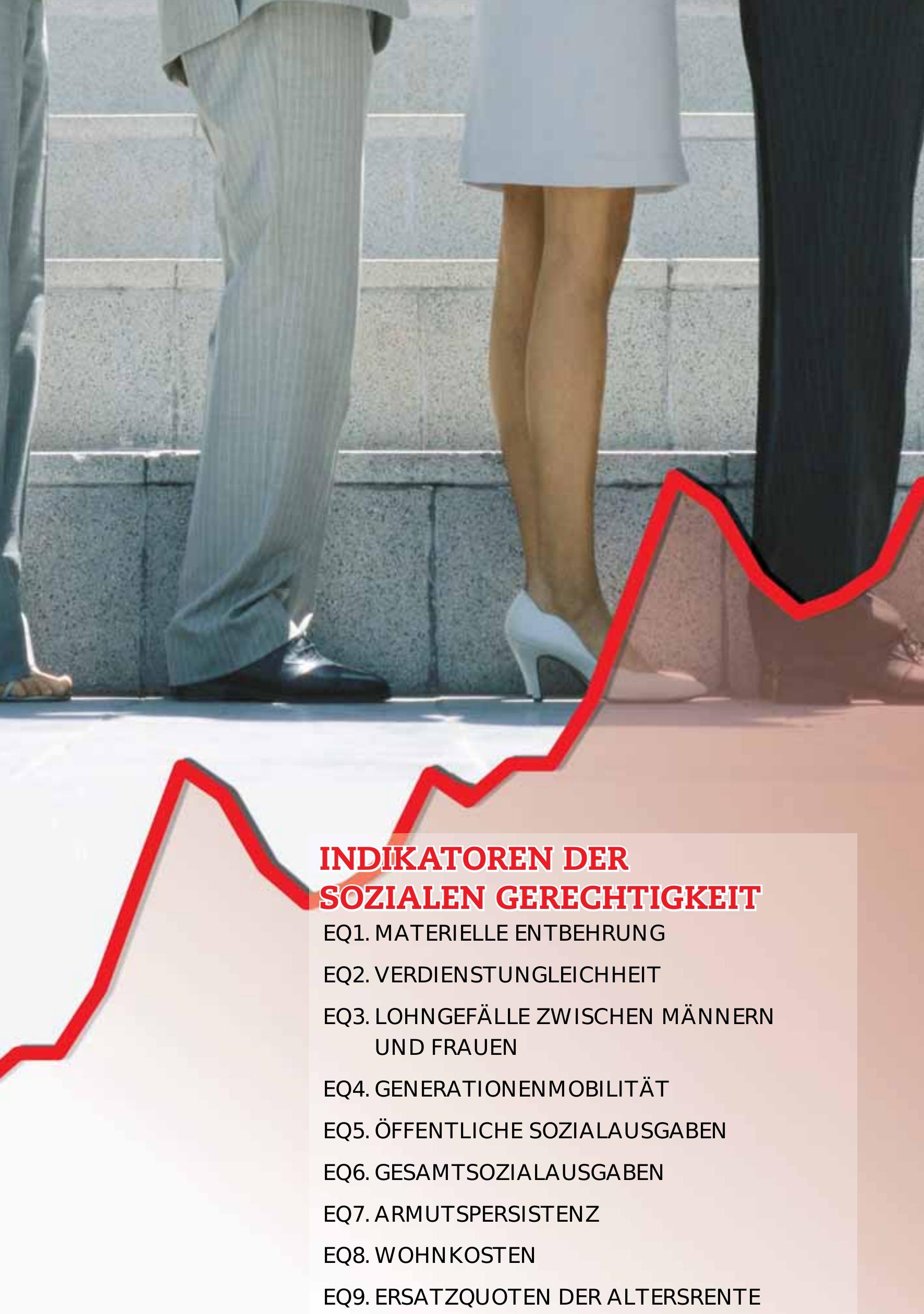




\section{Definition und Messung}

Messgrößen der materiellen Entbehrung ergänzen das Bild der Armutssituation, das anhand konventioneller Einkommensmessgrößen gezeichnet werden kann. Mit materieller Entbehrung ist die Unfähigkeit einer Person bzw. eines Haushalts gemeint, die Konsumgüter und Aktivitäten zu finanzieren, die in einer Gesellschaft zu einem bestimmten Zeitpunkt üblich sind, ohne Berücksichtigung der individuellen Präferenzen in Bezug auf diese Güter oder Aktivitäten. Für eine Reihe von Ländern können Indikatoren der materiellen Entbehrung Haushaltserhebungen entnommen werden, am Einkommen orientierte Armutsmaße liegen jedoch für mehr Länder vor.

Die nachstehenden Daten beziehen sich auf den Anteil der Haushalte, die sich bestimmte Güter und Aktivitäten laut eigener Angabe nicht leisten können. Daraus lässt sich in zwei Schritten ein einfacher summarischer Indikator der materiellen Entbehrung ableiten. Nach Erfassung von Daten zur Häufigkeit bestimmter Formen der Deprivation, die in sechs große Kategorien eingeteilt sind (Grundbedarf, grundlegende Freizeitaktivitäten, Gebrauchsgüter, Wohnbedingungen, finanzielle Belastung und Abhängigkeit von der Unterstützung durch andere) wird zunächst für jede dieser sechs Kategorien ein Durchschnittswert berechnet. Anschließend wird aus diesem einfachen Durchschnitt der sechs Aggregate ein summarischer Gesamtindex konstruiert. Die Vergleichbarkeit der Daten der verschiedenen Länder wird durch die unterschiedliche Formulierung der Fragebogen, durch abweichende Erhebungsmerkmale (z.B. Stichprobengröße, Heranziehung von Proxy-Befragten usw.) sowie möglicherweise in manchen Ländern durch fehlende Angaben zu bestimmten Gütern beeinträchtigt. Detailliertere Informationen zu diesen Messgrößen sind Boarini und Mira d'Ercole (2006) zu entnehmen.

In allen OECD-Ländern sieht sich ein signifikanter Teil der Haushalte laut eigenen Angaben mit verschiedenen Formen materieller Entbehrung konfrontiert. Tabelle EQ1.1 liefert Informationen zu einigen dieser Formen. Anfang dieses Jahrzehnts waren in den OECD-Ländern, die in der Tabelle EQ1.1 erfasst sind, insgesamt rd. 10\% der privaten Haushalte nicht in der Lage, ihren Grundbedarf zu decken - d.h. ihre Wohnung ausreichend zu heizen, gesund zu essen oder Zugang zu Gesundheitsversorgung zu erlangen -, und rund ein Drittel konnte es sich nicht leisten, in den vorangegangenen zwölf Monaten wenigstens eine Woche in Urlaub zu fahren. Was die Gebrauchsgüter anbelangt, verfügten nur wenige Haushalte nicht über einen Fernseher oder ein Telefon, aber rund ein Fünftel hatte keinen Computer. Zwar verfügen die meisten Haushalte in den genannten OECD-Ländern über eine Innentoilette, aber jeder zehnte Haushalt gab an, dass das Haus reparaturbedürftig, und $13 \%$, dass es Umweltbelastungen ausgesetzt sei. Weniger als $10 \%$ der Haushalte waren laut eigener Angaben im vorangegangenen Jahr in Zahlungsrückstand geraten, aber immerhin $20 \%$ gaben an, dass sie nur mit Not über die Runden kommen bzw. gelegentlich nicht in der Lage sind, wichtige Ausgaben zu tätigen.

In Bezug auf die Verbreitung materieller Entbehrung bestehen große Unterschiede zwischen den Ländern. Laut einem summarischen Index der materiellen Entbehrung - dem einfachen Durchschnitt der Entbehrungsindikatoren der sechs in Tabelle EQ1.1 behandelten Kategorien - sind in Griechenland, Polen, der Türkei und Ungarn über 20\% der Haushalte mit verschiedenen Formen von Entbehrung konfrontiert, wohingegen dieser Anteil in Dänemark, Luxemburg und Schweden nur 5\% beträgt. Bei Betrachtung aller OECD-Länder, für die Daten vorliegen, lässt sich nur ein schwacher Zusammenhang zwischen dem summarischen Index der materiellen Entbehrung und der Verbreitung von
Einkommensarmut feststellen (die anhand einer bei der Hälfte des Medianeinkommens angesetzten Armutsschwelle gemessen wird), im Verhältnis zum Pro-Kopf-BIP ist die Korrelation jedoch stärker (Abb. EQ1.2). Bei Nichtberücksichtigung der OECD-Länder, in denen das Pro-Kopf-BIP unter 25000 US-\$ liegt, ist allerdings das Gegenteil der Fall, d.h. es besteht eine stärkere Korrelation mit der relativen Einkommensarmut und eine schwächere mit dem durchschnittlichen Pro-Kopf-Einkommen. Dies lässt darauf schließen, dass dieser einfache Index der materiellen Entbehrung sowohl Informationen über den absoluten Lebensstandard als auch über den Lebensstandard im unteren Bereich der Einkommensverteilung der einzelnen Länder liefert.

Nationale Untersuchungen des Grads der Konfrontation der privaten Haushalte mit verschiedenen Formen materieller Entbehrung zeigen zugleich durchgehend, dass zwischen mehrfacher Entbehrung und Einkommensarmut überraschenderweise nur relativ geringe Überschneidungen bestehen - selbst wenn die Entbehrungsgrenze so angesetzt ist, dass sie die gleichen Armutszahlen liefert wie eine am Einkommen orientierte Armutsschwelle (vgl. z.B. Perry, 2002) - und dass sich die Zusammensetzung der Gruppe der Einkommensarmen deutlich von der der Gruppe der Haushalte unterscheidet, die unter materieller Entbehrung leiden (selbst bei Verwendung longitunaler Messgrößen beider Phänomene, vgl. z.B. Whelan et al., 2004).

Statusindikatoren: Generationenmobilität (EQ4), Armutspersistenz (EQ7), Arbeitslosigkeit (SS2).

Reaktionsindikatoren: Öffentliche Sozialausgaben (EQ5), Leistungen bei Nichterwerbstätigkeit (SS6), Gesundheitsausgaben (HE2). 


\section{EQ1.1 Ein signifikanter Teil der Haushalte ist laut eigenen Angaben mit verschiedenen Formen materieller Entbehrung konfrontiert}

Anteil der Haushalte, die laut eigenen Angaben unter verschiedenen Formen materieller Entbehrung leiden, um das Jahr 2000

\begin{tabular}{|c|c|c|c|c|c|c|c|c|c|c|c|c|c|}
\hline & \multicolumn{13}{|c|}{ Entbehrung in Bezug auf: } \\
\hline & \multicolumn{3}{|c|}{ Grundbedarf } & \multirow{2}{*}{\begin{tabular}{|c}
$\begin{array}{c}\text { Freizeit- } \\
\text { aktivitäten }\end{array}$ \\
$\begin{array}{c}1 \text { Woche } \\
\text { Urlaub } \\
\text { auswärts } \\
\text { pro Jahr }\end{array}$
\end{tabular}} & \multicolumn{3}{|c|}{ Gebrauchsgüter } & \multicolumn{3}{|c|}{ Wohnraum } & \multicolumn{2}{|c|}{$\begin{array}{l}\text { Finanzielle } \\
\text { Belastung }\end{array}$} & \multirow{2}{*}{\begin{tabular}{|c|}
$\begin{array}{c}\text { Unterstüt- } \\
\text { zungs- } \\
\text { bedarf }\end{array}$ \\
$\begin{array}{c}\text { Regelmäßige } \\
\text { Unterstüt- } \\
\text { zung durch } \\
\text { Dritte }\end{array}$ \\
\end{tabular}} \\
\hline & Heizung & $\begin{array}{l}\text { Gesundes } \\
\text { Essen }\end{array}$ & $\begin{array}{l}\text { Gesund- } \\
\text { heitsver- } \\
\text { sorgung }\end{array}$ & & Fernseher & Telefon & $P C$ & $\begin{array}{l}\text { Repara- } \\
\text { turbedarf }\end{array}$ & $\begin{array}{l}\text { Keine } \\
\text { Innen- } \\
\text { toilette }\end{array}$ & $\begin{array}{l}\text { Umwelt- } \\
\text { belas- } \\
\text { tungen }\end{array}$ & $\begin{array}{l}\text { Zahlungs- } \\
\text { rück- } \\
\text { stände }\end{array}$ & $\begin{array}{c}\text { Ständige } \\
\text { Geld- } \\
\text { knapp- } \\
\text { heit }\end{array}$ & \\
\hline Australien & 2 & 12 & $\ldots$ & 26 & 1 & 4 & 32 & 8 & 1 & $\ldots$ & 15 & $\ldots$ & 10 \\
\hline Belgien & 4 & 3 & 8 & 20 & 0 & 1 & 5 & 6 & 2 & 10 & 5 & 11 & 7 \\
\hline Dänemark & 2 & 1 & 1 & 11 & 0 & 0 & 5 & 5 & 0 & 4 & 2 & 11 & 10 \\
\hline Deutschland & 3 & 2 & 3 & 21 & 0 & 1 & 18 & 7 & 1 & 5 & 4 & 9 & 8 \\
\hline Finnland & 7 & 4 & 3 & 26 & 1 & 0 & 8 & 2 & 1 & 14 & 6 & 12 & 13 \\
\hline Frankreich & 4 & 3 & 4 & 24 & 0 & 1 & 11 & 9 & 2 & 17 & 5 & 12 & 9 \\
\hline Griechenland & 31 & 26 & 21 & 51 & 2 & 2 & 16 & 9 & 6 & 15 & 21 & 49 & 19 \\
\hline Irland & 4 & 1 & 10 & 24 & 1 & 2 & 15 & 5 & 1 & 7 & 3 & 10 & 8 \\
\hline Italien & 17 & 5 & 26 & 36 & 1 & 1 & 15 & 6 & 1 & 15 & 3 & 22 & 6 \\
\hline Japan & 1 & . & 2 & 26 & . & 2 & 12 & 17 & 1 & . & 5 & 25 & 10 \\
\hline Kanada & $\ldots$ & 8 & $\ldots$ & 0 & $\ldots$ & 4 & $\ldots$ & 8 & . & . & 14 & . & $\ldots$ \\
\hline Luxemburg & 6 & 2 & 5 & 8 & 0 & 0 & 2 & 6 & . & 16 & 3 & 7 & 6 \\
\hline Neuseeland & 4 & 11 & 8 & 21 & 0 & 2 & . & 14 & 0 & 7 & 10 & $\ldots$ & 14 \\
\hline Niederlande & 3 & 2 & 3 & 13 & 0 & 0 & 4 & 8 & 0 & 11 & 1 & 9 & 10 \\
\hline Österreich & 1 & 6 & 5 & 21 & 0 & 1 & 9 & 4 & 3 & 4 & 1 & 14 & 13 \\
\hline Polen & 30 & 17 & 19 & 68 & $\ldots$ & .. & 40 & 25 & 11 & 22 & 28 & 53 & 17 \\
\hline Portugal & 56 & 3 & 17 & 59 & 2 & $\ddot{5}$ & 26 & 23 & 7 & 19 & 1 & 34 & 12 \\
\hline Schweden & 1 & 2 & 3 & 15 & 0 & $\ldots$ & 4 & 4 & 1 & 5 & 4 & 5 & 0 \\
\hline Slowak. Rep. & 17 & 33 & 21 & 64 & . & $\cdots$ & 28 & 26 & 7 & 18 & 15 & 24 & 17 \\
\hline Spanien & 42 & 3 & 4 & 37 & 0 & 2 & 21 & 9 & 0 & 10 & 3 & 21 & 12 \\
\hline Tschech. Rep. & 8 & 19 & 3 & 34 & $\ldots$ & $\ldots$ & 18 & 9 & 5 & 20 & 7 & 19 & 14 \\
\hline Türkei & 45 & 53 & 33 & 66 & $\cdots$ & $\cdots$ & 61 & 20 & 12 & 29 & 26 & 48 & 19 \\
\hline Ungarn & 11 & 34 & 8 & 63 & . & . & 23 & 19 & 9 & 22 & 18 & 28 & 20 \\
\hline Ver. Königreich & 2 & 8 & 3 & 24 & 0 & 0 & 10 & 6 & 1 & 7 & 11 & 7 & 11 \\
\hline Ver. Staaten & 7 & 11 & 8 & .. & 1 & 5 & 33 & 5 & .. & 3 & 10 & 15 & 24 \\
\hline Enfacher Durchschnitt & 13 & 11 & 9 & 33 & 1 & 2 & 18 & 10 & 3 & 13 & 9 & 20 & 13 \\
\hline
\end{tabular}

. .: Daten nicht verfügbar.

Anmerkung: Die Angaben beziehen sich auf den Durchschnitt der Items der sechs aufgezeigten Formen materieller Entbehrung. Wegen der unterschiedlichen Datenlage kann die Zahl der in den verschiedenen Ländern berücksichtigten Items schwanken.

\section{EQ1.2 Mehr materielle Entbehrung in Ländern mit höherer relativer Einkommensarmut und geringerem Pro-Kopf-BIP} Um das Jahr 2000
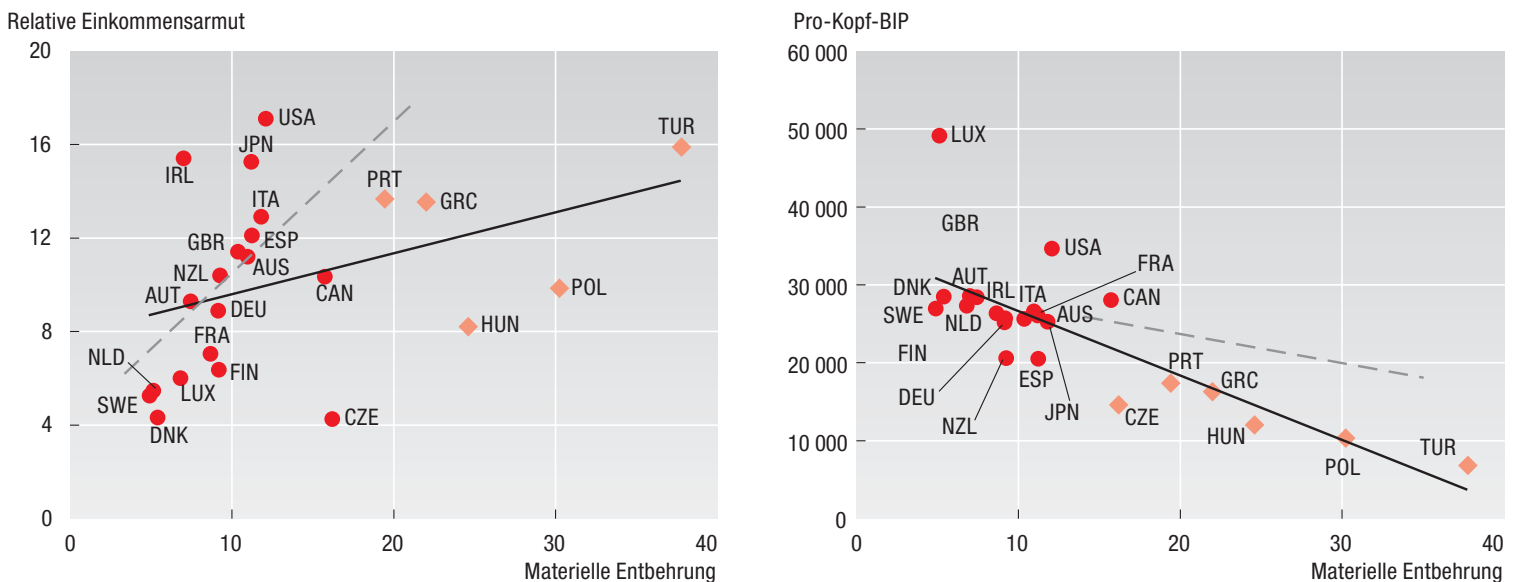

Anmerkung: Die materielle Entbehrung bezieht sich auf den Anteil der Haushalte, die verschiedene Formen der Entbehrung unter den sechs großen in Tabelle EQ1.1 aufgeführten Kategorien nannten, deren Durchschnitt ermittelt wird. Die relative Einkommensarmut wird anhand eines bei der Hälfte des verfügbaren Medianeinkommens angesetzten Schwellenwerts gemessen. OECD-Länder, in denen das Pro-Kopf-BIP unter 25000 US-\$ liegt, sind durch Rauten gekennzeichnet. Die graue gestrichelte Linie stellt in jedem Abbildungsteil jeweils den Trendverlauf zwischen den beiden Variablen dar, wenn die Analyse auf Länder mit einem Pro-Kopf-BIP von über 25000 US-\$ beschränkt wird.

Quelle: Boarini, R. und M. Mira d'Ercole (2006), "Measures of Material Deprivation in OECD Countries", OECD Social, Employment and Migration Working Paper, No. 37, Paris (ww w.oecd.org/els/w orkingpapers).

StatLink 2 http://dx.doi.org/10.1787/365101528828

Weitere Informationen a Perry, B. (2002), "The Mismatch between Income Measures and Direct Outcome Measures of Poverty”, Social Policy Journal of New Zealand, Vol. 19, S. 101-127. Whelan, C., R. Layte und B. Maitre (2004), “Understanding the Mismatch Between Income Poverty and Deprivation: A Dynamic Comparative Analysis", European Sociological Review, Vol. 20, No. 4. 


\section{Definition und Messung}

Die Verdienstungleichheit kann anhand einer Vielzahl von Daten gemessen werden. Beim hier verwendeten Indikator handelt es sich um die "Dezil-Ratio", die man erhält, wenn man die Verdienste im obersten und im untersten Dezil der Verteilung (d.h. der 10\% der Arbeitskräfte mit dem höchsten und mit dem niedrigsten Verdienst) mit dem Medianverdienst vergleicht (der Grenze, die die Arbeitskräfte in zwei gleich große Gruppen teilt). In diesem Abschnitt steht D9 für die obere Grenze des 9. Dezils der Verdienstverteilung (die der Untergrenze des obersten Dezils entspricht), während D1 die Obergrenze des untersten Dezils und D5 der Medianverdienst ist.

Die nachstehen den Angaben beziehen sich im Allgemeinen auf Vollzeitkräfte. Die Verdienste werden brutto gemessen, d.h. vor Abzug von Einkommensteuer und Arbeitnehmerbeiträgen zur Sozialversicherung. Sie umfassen Grundgehälter und -löhne, Überstundenzulagen, Prämien und Gratifikationen, zusätzliche monatliche Zahlungen sowie regelmäßige oder gelegentliche Nebenleistungen; u.U. nicht enthalten sind hingegen Teile des Vergütungspakets von Managern und sonstigen Führungskräften, wie z.B. Aktienoptionen. Die Daten aus der OECD Earnings Database stammen aus verschiedenen nationalen Quellen (Haushaltserhebungen, Betriebspanels und Verwaltungsunterlagen), so dass Abweichungen in Bezug auf die erfassten Verdienstkomponenten, den Bezugszeitraum (Jahr, Monat, Tag, Stunde) und die Behandlung sehr hoher Verdienste in erhebungsbasierten Schätzungen (top-coding) nicht auszuschließen sind. Diese Abweichungen können sich auf die Beurteilung von im Ländervergleich festzustellenden Unterschieden bei der Verdienstverteilung und deren Veränderung im Zeitverlauf auswirken.

Die seit Anfang der neunziger Jahre zu beobachtenden Trends bei der Verdienstungleichheit unterscheiden sich im oberen und unteren Teil der Verdienstverteilung. Was erstere Gruppe anbelangt, hat sich das Verhältnis D9:D5 in den meisten OECD-Ländern geringfügig, aber stetig erhöht. Der durchschnittliche Anstieg belief sich in den 11 OECDLändern, für die Daten aus dem gesamten Zeitraum vorliegen, auf über $4 \%$, in Australien, Dänemark und Schweden betrug er jedoch fast $10 \%$ oder mehr, wohingegen sich die entsprechende Relation in Japan leicht verringerte. In der unteren Hälfte der Verteilung waren die Veränderungen jedoch unerheblich. Das Verhältnis D5:D1 erhöhte sich im Durchschnitt leicht, verringerte sich in fünf OECD-Ländern und stieg nur in Dänemark, Deutschland und Schweden in stärkerem Maße (um 5\% oder mehr).

In Bezug auf den Grad der Verdienstungleichheit sind zwischen den Ländern ebenfalls einige deutliche Unterschiede festzustellen. Die Verdienstungleichheit unter den Vollzeitkräften ist erwartungsgemäß in den meisten OECDLändern im oberen Teil der Verteilung höher als im unteren (nicht jedoch in Kanada, Korea und der Schweiz). In den neunziger Jahren und Anfang dieses Jahrzehnts bewegten sich die Dezil-Ratios D9:D5 und D5:D1 in allen in Tabelle EQ2.1 dargestellten Ländern außer den Vereinigten Staaten bei unter zwei.

Im Ländervergleich zu beobachtende Unterschiede bei der Verdienstungleichheit im unteren Teil der Verteilung sind z.T. auf die gesetzlichen Mindestlöhne zurückzuführen, die in einigen Ländern für die Mehrzahl der Arbeitskräfte gelten. Diese Mindestlöhne wirken sich auf die Verdienstungleichheit aus, indem sie ein Mindestniveau für die Arbeitsentgelte von Geringverdienern festlegen, selbst wenn dadurch einige Arbeitskräfte (z.B. die mit den geringsten Qualifikationen) vom Arbeitsmarkt verdrängt werden, weil sie zu teuer sind. Die Angaben in Abbildung EQ2.2 beziehen sich auf die gesetzlichen Mindestlöhne für Erwachsene im Verhältnis zum Medianverdienst von Vollzeitkräften. 2003 schwankte dieses Verhältnis stark im OECD-Ländervergleich, es reichte von 30\% oder weniger in Mexiko, Korea und Spanien bis zu 50\% oder mehr in Frankreich, Australien, Luxemburg und den Niederlanden. In Irland und im Vereinigten Königreich, wo Ende der neunziger Jahre landesweite Mindestlöhne eingeführt wurden, beträgt dieses Verhältnis rd. 40\%. Seit 1980 sind die Mindestverdienste im Verhältnis zu den Medianverdiensten im Durchschnitt von $50 \%$ auf $43 \%$ gesunken, in Frankreich und anderen kontinentaleuropäischen Ländern sind sie jedoch gestiegen.

Statusindikatoren: Generationenmobilität (EQ4), Armutspersistenz (EQ7), Beschäftigung (SS1).

Reaktionsindikatoren: Öffentliche Sozialausgaben (EQ5), Leistungen bei Erwerbslosigkeit (SS6). 


\section{EQ2.1 Mäßiger Anstieg der Verdienstungleichheit in der oberen Hälfte der Verteilung, Stabilität in der unteren Hälfte}

Dezil-Ratios, Bruttoverdienste von Vollzeitkräften

\begin{tabular}{|c|c|c|c|c|c|c|c|c|}
\hline & \multicolumn{4}{|c|}{ Verhältnis D9:D5 } & \multicolumn{4}{|c|}{ Verhältnis D5:D1 } \\
\hline & $1990^{1}$ & $1995^{2}$ & $2000^{3}$ & $2003^{4}$ & $1990^{1}$ & $1995^{2}$ & $2000^{3}$ & $2003^{4}$ \\
\hline Australien & 1.7 & 1.8 & 1.8 & 1.9 & 1.7 & 1.6 & 1.7 & 1.6 \\
\hline Belgien & . & . & 1.5 & 1.5 & $\ldots$ & $\ldots$ & 1.3 & 1.4 \\
\hline Dänemark & 1.6 & 1.7 & 1.7 & 1.8 & 1.4 & 1.4 & 1.4 & 1.5 \\
\hline Deutschland & 1.7 & 1.8 & 1.8 & 1.8 & 1.6 & 1.6 & 1.6 & 1.7 \\
\hline Finnland & 1.7 & 1.7 & 1.7 & 1.7 & 1.5 & 1.4 & 1.4 & 1.4 \\
\hline Japan & 1.9 & 1.8 & 1.8 & 1.8 & 1.7 & 1.6 & 1.6 & 1.6 \\
\hline Kanada & . & $\ldots$ & 1.8 & 1.9 & $\ldots$ & . & 2.0 & 2.0 \\
\hline Korea & 2.1 & 1.9 & 1.9 & 2.0 & 1.9 & 1.9 & 2.1 & 2.0 \\
\hline Neuseeland & . & $\ldots$ & 1.7 & 1.8 & .. & $\ldots$ & 1.5 & 1.6 \\
\hline Norwegen & $\ldots$ & . & 1.4 & 1.5 & . & . & 1.4 & 1.4 \\
\hline Polen & 1.8 & 2.0 & 2.0 & . & 1.7 & 1.7 & 1.8 & .. \\
\hline Portugal & . & . & 2.1 & 2.2 & . & . & 1.5 & 1.4 \\
\hline Schweden & 1.5 & 1.6 & 1.7 & 1.7 & 1.3 & 1.4 & 1.4 & 1.4 \\
\hline Tschech. Rep. & $\ldots$ & 1.7 & 1.8 & 2.3 & $\ldots$ & 1.6 & 1.7 & 1.7 \\
\hline Ver. Königreich & 1.9 & 1.9 & 1.9 & 2.0 & 1.8 & 1.8 & 1.8 & 1.8 \\
\hline Ver. Staaten & 2.1 & 2.2 & 2.2 & 2.3 & 2.0 & 2.1 & 2.0 & 2.1 \\
\hline OECD11 & 1.8 & 1.8 & 1.9 & 1.9 & 1.7 & 1.7 & 1.7 & 1.7 \\
\hline
\end{tabular}

Anmerkung: Die Daten beziehen sich auf Vollzeitkräfte. D9:D5 ist das Verhältnis zwischen der Obergrenze der Verdienste der Arbeitnehmer im 9. Dezil der Verdienstverteilung und dem Medianverdienst. D5:D1 ist das Verhältnis zwischen dem Medianverdienst und der Obergrenze der Verdienste der Arbeitnehmer im 1. Dezil der Verdienstverteilung.

1991 für die Schweiz.

2. 1996 für die Tschechische Republik.

3. 1999 für die Tschechische Republik und Polen; 2001 für Portugal.

4. 2002 für Finnland, Frankreich, Deutschland, Korea, Norwegen, Spanien und OECD11.

Quelle: OECD Earnings database.

\section{EQ2.2 Die Mindestlöhne sinken im Durchschnitt im Vergleich zum Medianverdienst}

Mindestlöhne im Verhältnis zum M edianverdienst erwachsener Vollzeitkräfte, 1980, 1990 und 2003

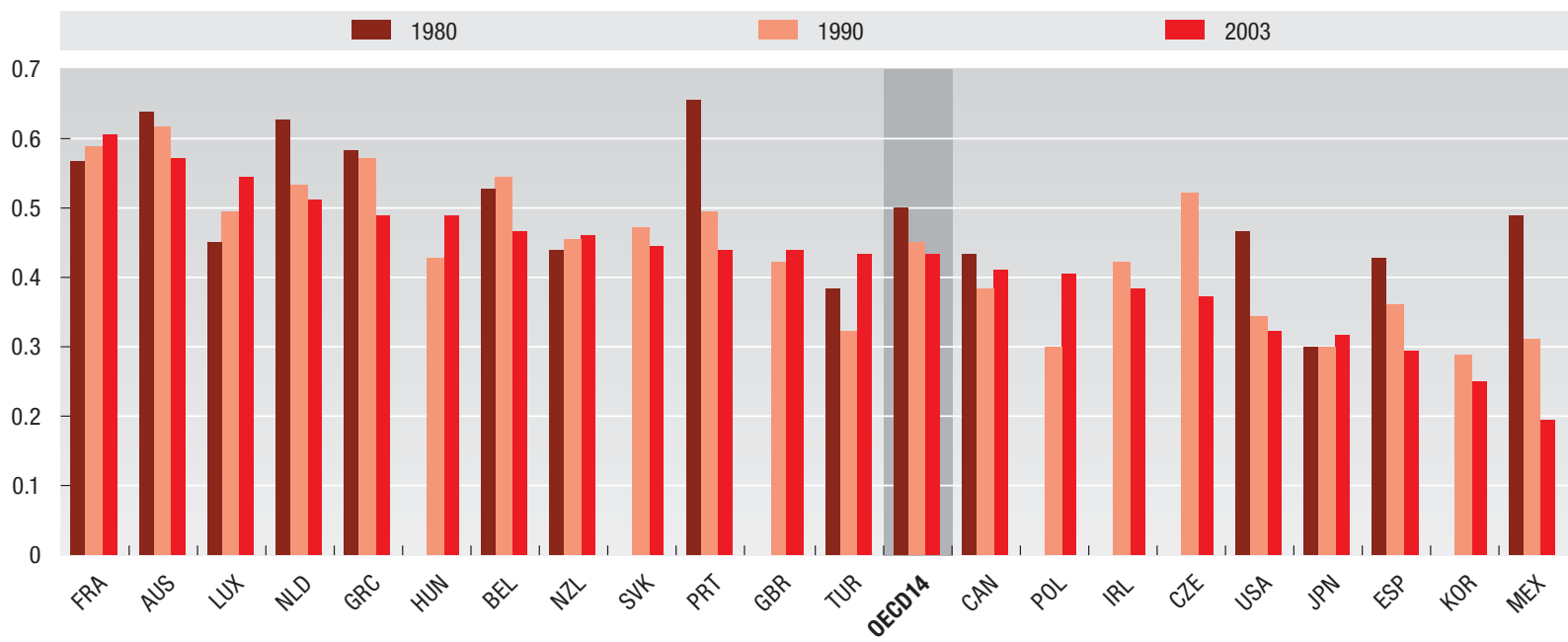

Anmerkung: Die Angaben beziehen sich auf die in Gesetzen, Verordnungen oder Tarifverträgen bzw. -abkommen festgelegten Mindestlöhne für Erwachsene, die effektiv landesweit gültig sind. Die Länder sind von links nach rechts in absteigender Reihenfolge nach der Höhe des Mindestlohns im Verhältnis zum Medianlohn von Vollzeitkräften im Jahr 2003 dargestellt. Im Fall Australiens beziehen sich die Angaben auf den nationalen Mindestlohn, der speziell für im Rahmen nationaler Tarifabkommen beschäftigte Arbeitskräfte eingeführt wurde, im Allgemeinen aber auch für nach den Tarifverträgen der einzelnen Bundesstaaten beschäftigte Arbeitskräfte und somit für die überwiegende Mehrzahl der Arbeitskräfte gilt, vor allem für Geringverdiener, die mit größter Wahrscheinlichkeit von Mindestlohnregelungen betroffen sind.

Quelle: OECD Minimum Wages database.

St tatLink 2 http://dx.doi.org/10.1787/035182557721

Weitere Informationen n OECD (1996), "Earnings Inequality, Low-paid Employment and Earnings Mobility", OECD Employment Outlook, Paris, Juni. OECD (1998), "Making the Most of the Minimum: Statutory Minimum Wages, Employment and Poverty", OECD Employment Outlook, Paris, Juni. 


\section{Definition und Messung}

Geschlechtsspezifische Lohnunterschiede liefern einen Anhaltspunkt für den Grad der Erwerbseinkommensgleichheit bzw. -ungleichheit zwischen Männern und Frauen. Das Lohngefälle zwischen den Geschlechtern wird hier an der Differenz zwischen dem Medianverdienst von männlichen und weiblichen Arbeitskräften gemessen, ausgedrückt in Prozent des Medianverdiensts von vollzeitbeschäftigten Männern. Darüber hinaus wird es auch für niedrige und hohe Verdienstniveaus gemessen (20. bzw. 80. Perzentil).

Die Angaben zum Lohngefälle zwischen Männern und Frauen stammen aus der OECD Earnings Database. Wie schon im Abschnitt zur Verdienstungleichheit erwähnt, können die entsprechenden Messgrößen auf Grund unterschiedlicher Methoden zur Messung von Vollzeitverdiensten nicht als ein genauer Indikator für Unterschiede zwischen den Ländern betrachtet werden. Außerdem sind Diskrepanzen in Bezug auf die Zahl der von Vollzeitkräften geleisteten Arbeitsstunden in dieser Messung nicht berücksichtigt.

Das geschlechtsspezifische Lohngefälle spielt eine besonders wichtige Rolle angesichts der Notwendigkeit, die Erwerbsbeteiligung der Frauen zu erhöhen und Chancengleichheit zu verwirklichen. In den OECD-Ländern sind immer noch erhebliche Lohnunterschiede zwischen Männern und Frauen festzustellen. Das geschlechtsspezifische Lohngefälle beim Median beträgt im Durchschnitt von 21 OECD-Ländern 18\% (Abb. EQ3.1). Dabei fallen Unterschiede zwischen den Ländern auf. Das geschlechtsspezifische Lohngefälle schwankt zwischen nur $6 \%$ bzw. $9 \%$ in Neuseeland und Belgien und nicht weniger als $30 \%$ bzw. $41 \%$ in Japan und Korea. Ein großer Teil des geschlechtsspezifischen Lohngefälles innerhalb der einzelnen Länder ebenso wie ein Teil der Varianz zwischen den Ländern kann auf Geschlechtsunterschiede in der Zusammensetzung der Erwerbsbevölkerung zurückgeführt werden. Der Gesamtumfang der Lohnungleichheit in den einzelnen Ländern hat ebenfalls Einfluss auf die Varianz des geschlechtsspezifischen Lohngefälles im Ländervergleich und könnte dieses zu einem großen Teil erklären (Blau und Kahn, 2001). Auch mutterschaftsbedingte Unterbrechungen der beruflichen Laufbahn schlagen sich im Lohngefälle zwischen Männern und Frauen nieder.

In den meisten Ländern ist das Lohngefälle zwischen den Geschlechtern im oberen Einkommensspektrum (beim 80. Perzentil) höher als im unteren (beim 20. Perzentil) (Abb. EQ3.2). Dabei gibt es jedoch Ausnahmen - Vereinigtes Königreich, Schweiz und Portugal -, und in Dänemark wie auch Belgien sind die Unterschiede nicht signifikant. Das geringere geschlechtsspezifische Lohngefälle im unteren Bereich der Verdienstverteilung könnte auf institutionelle Faktoren zurückzuführen sein, wie den Einfluss der Mindestlöhne oder der Tarifbindung (Blau und Kahn, 2001). Das höhere Lohngefälle zwischen Männern und Frauen im oberen Verdienstspektrum wird häufig als ein Zeichen für das Vorhandensein eines sogenannten "Glass Ceiling", einer unsichtbaren Barriere, gesehen (Arulampalam et al., 2006).

Infolge des im Vergleich zu den Männern steigenden Aus- und Weiterbildungsniveaus der Frauen und ihrer wachsenden Arbeitsmarktbindung hat sich der Umfang des geschlechtsspezifischen Lohngefälles in den meisten Ländern, für die Daten vorliegen, im Zeitverlauf tendenziell verringert (Tabelle EQ3.3). Die stärkste Abnahme war seit Anfang der achtziger Jahre in den Vereinigten Staaten zu verzeichnen, wo das geschlechtsspezifische Lohngefälle zuvor relativ hoch war. Auch in Japan und im Vereinigten Königreich ist das geschlechtsspezifische Lohngefälle im Zeitverlauf stetig gesunken. In Frankreich und Österreich steigt es inzwischen hingegen. In Frankreich kam es damit zu einer Trendwende, nachdem sich das Lohngefälle lange verringert hatte. In der Mehrzahl der OECD-Länder sind wechselnde Trends zu beobachten.

Trotz der Bestimmungen, die "gleiche Bezahlung für gleiche Arbeit" verlangen, und der in den meisten OECDLändern geltenden Antidiskriminierungsgesetze ist ein Teil des geschlechtsspezifischen Lohngefälles in allen Ländern auf eine Diskriminierung der Frauen auf dem Arbeitsmarkt zurückzuführen. Weil aber Diskriminierung nur selten direkt beobachtbar ist und zudem andere Messprobleme auftreten, lässt sich schwer genau bestimmen, in welchem Umfang die Diskriminierung zum geschlechtsspezifischen Lohngefälle innerhalb der einzelnen Länder und im Ländervergleich beiträgt. Eine indirekte, grobe Messgröße der Diskriminierung ist die "nicht erklärbare" Lohndifferenz. Durch den Vergleich der Arbeitsentgelte von Männern und Frauen in sehr ähnlichen Beschäftigungen oder die Gegenüberstellung der Arbeitsentgelte mit spezifischen Messgrößen der Produktivität konnten einige Untersuchungen stichhaltige Belege für eine Ungleichbehandlung von Männern und Frauen liefern.

Statusindikatoren: Verdienstungleichheit (EQ2), Erwerbstätige Mütter (SS3). 


\section{EQ3.1 Frauen verdienen weniger als Männer}

Differenz zwischen dem M edianverdienst von männlichen und weiblichen Vollzeitkräften, 2004 oder letztes verfügbares Jahr, in Prozent

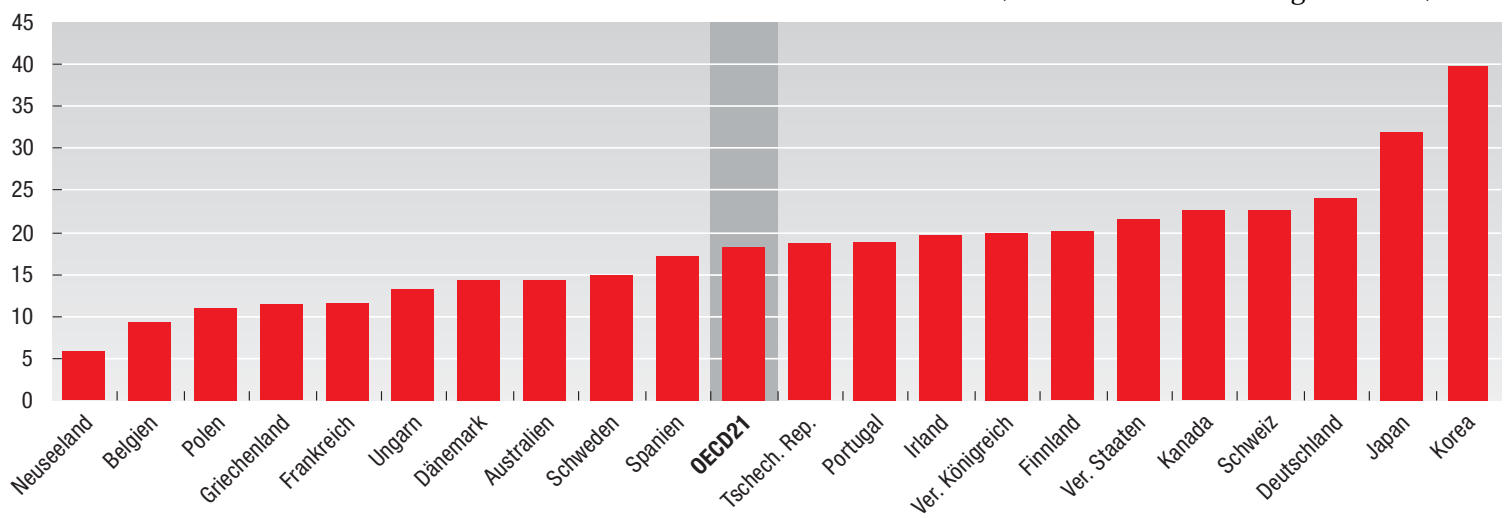

Anmerkung: Die Länder sind von links nach rechts in aufsteigender Reihenfolge nach der Höhe des geschlechtsspezifischen Lohngefälles dargestellt.

EQ3.2 Höhere Lohngefälle zwischen Männern und Frauen im oberen Verdienstspektrum

Differenz zwischen dem Vollzeitverdienst von Männern und Frauen am oberen und unteren Ende der Verdienstverteilung, 2003 oder letztes verfügbares Jahr

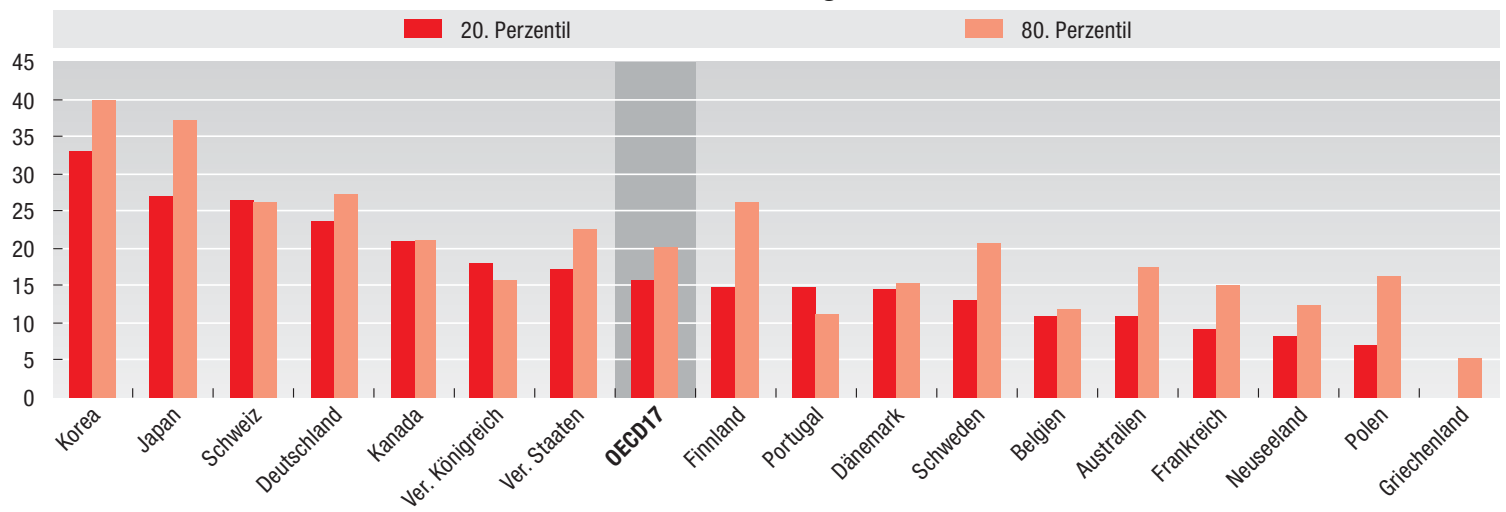

Anmerkung: Die Länder sind von links nach rechts in absteigender Reihenfolge nach der Höhe des geschlechtsspezifischen Lohngefälles im unteren, d.h. 20. Perzentil der Arbeitskräfte dargestellt.

EQ3.3 In den meisten Ländern verringert sich das Lohngefälle zwischen Männern und Frauen Differenz zwischen dem M edianverdienst von männlichen und w eiblichen Vollzeitkräften

\begin{tabular}{|c|c|c|c|c|c|c|c|c|c|c|c|c|c|}
\hline & 1980 & $1985^{1}$ & $1990^{2}$ & $1995^{3}$ & $2000^{4}$ & $2004^{5}$ & & 1980 & $1985^{1}$ & $1990^{2}$ & $1995^{3}$ & $2000^{4}$ & $2004^{5}$ \\
\hline Australien & 18.8 & 19.6 & 18.2 & 14.5 & 17.2 & 14.4 & Korea & $\ldots$ & 51.9 & & 43.1 & 40.7 & 39.8 \\
\hline Belgien & . & $\ldots$ & . & . & 8.8 & 9.3 & Niederlande & $\ldots$ & 25.6 & 25.0 & 23.1 & 21.7 & $\ldots$ \\
\hline Dänemark & . & $\ldots$ & $\ldots$ & 14.1 & 14.7 & 12.3 & Österreich & 35.1 & . & 32.2 & 31.2 & 32.9 & . \\
\hline Deutschland & $\ldots$ & 27.0 & 27.2 & 23.5 & 23.0 & 24.1 & Polen & $\ldots$ & $\ldots$ & 19.9 & 19.9 & 16.8 & 11.0 \\
\hline Fnnland & 26.6 & 22.5 & 22.9 & 22.4 & 20.4 & 20.1 & Schweden & 14.5 & 18.4 & 19.6 & 19.0 & 15.5 & 14.8 \\
\hline Frankreich & 19.7 & 17.0 & 15.3 & 10.3 & 10.8 & 11.7 & Schweiz & $\ldots$ & . & 26.4 & 25.5 & 25.7 & 22.6 \\
\hline Irland & . & $\ldots$ & $\ldots$ & . & 19.7 & . & Tschech. Rep. & $\ldots$ & $\ldots$ & . & 21.1 & 21.8 & 18.6 \\
\hline Italien & $\ldots$ & 18.8 & 19.5 & 17.1 & . & . & Ungarn & $\ldots$ & $\ldots$ & $\ldots$ & 17.8 & 13.2 & . \\
\hline Japan & 41.7 & 41.7 & 40.6 & 37.1 & 33.9 & $\ldots$ & Ver. Königreich & 35.3 & 33.6 & 31.2 & 26.6 & 24.0 & 20.0 \\
\hline Kanada & $\ldots$ & . & . & . & 24.0 & 22.6 & Ver. Staaten & 36.6 & 33.0 & 28.5 & 24.6 & 24.5 & 21.6 \\
\hline
\end{tabular}

.: Daten nicht verfügbar.

1. 1986 für Finnland und Italien.

2. 1991 für Polen und Schweiz.

3. 1996 für Dänemark, Tschechische Republik und Ungarn.

4. 1999 für Niederlande, Österreich und Polen.

5. 2003 für Belgien, Finnland, Schweiz, Tschechische Republik, Vereinigtes Königreich und Vereinigte Staaten; 2002 für Deutschland, Frankreich, Korea und Polen.

Quelle: OECD Earnings database.

StatLink $2 \quad$ http://dx.doi.org/10.1787/364104645170

Weitere Informationen arulampalam, W, A. Booth und M. L. Bryan (2006), “Is There a Glass Ceiling over Europe? Exploring the Gender Pay Gap across the Wages Distribution", Discussion Paper, No. 510, Centre for Economic Policy Research, Research School of Social Sciences, Australian National University. Barth, E., M. Røed und H. Torp (2002), “Towards a Closing of the Gender Pay Gap. A comparative study of three occupations in six European countries", Institute for Social Research and the Norwegian Centre for Gender Equality, Oslo. Blau, F.D. und L.M. Kahn (2001), “Understanding International Differences in the Gender Pay Gap”, NBER Working Paper, No. W8200, Cambridge, MA (http://ssrn.com/abstract=265295). 


\section{Definition und Messung}

Generationenmobilität wird definiert als das Ausmaß, in dem sich Personen in Bezug auf eine Reihe von Schlüsselmerkmalen und Ergebnissen von ihren Eltern unterscheiden. Verschiedene Analyserichtungen haben sich dabei für unterschiedliche Arten von Indikatoren interessiert. Die wirtschaftswissenschaftliche Literatur befasste sich vor allem mit Bewegungen zwischen verschiedenen Einkommens- (oder Verdienst-) Klassen bzw. Perzentilen der Einkommensverteilung. Die soziologische Literatur untersuchte in erster Linie Bewegungen zwischen verschiedenen Berufsgruppen, die entsprechend ihres Ansehens bzw. der Gesellschaftsschicht, der sie zugeordnet werden können, eingestuft werden.

Hier wird als Hauptmessgröße der Generationenmobilität die intergenerative Verdienstelastizität verwendet, mit der der Anteil der Verdienstunterschiede zwischen den Vätern gemessen wird, der im Durchschnitt an deren Söhne weitergegeben wird (je geringer die Elastizität, umso höher ist die intergenerative Mobilität). Die Vergleichbarkeit dieser Schätzungen wird zwar durch eine Reihe von Faktoren eingeschränkt, hier sind jedoch die "bevorzugten" Schätzungen von Corak (2006) wiedergegeben, die sich auf eine Metaanalyse nationaler Untersuchungen stützen, in der verschiedene Faktoren berücksichtigt sind (Altersunterschiede zwischen Vätern und Söhnen, Dauer des Verdienstbeobachtungszeitraums, verwendete Methoden) und die von D'Addio (2006) mit Daten aus Australien, Italien und Spanien verknüpft wurde. Dieser Indikator wird durch Informationen über vom familiären Hintergrund abhängige Unterschiede bei den Bildungsergebnissen (in Mathematik) von 15-jährigen Schülerinnen und Schülern ergänzt, gestützt auf Daten aus der 2. Erhebung der Internationalen Schulleistungsstudie PISA der OECD.

Der Grad des Ressourcentransfers von den Eltern an ihre Kinder ist ein Maß der Chancengleichheit, die wiederum als ein Indikator für den Grad der Offenheit einer Gesellschaft betrachtet werden kann. Die Komplexität der Beurteilung der Generationenmobilität des sozioökonomischen Status hängt großenteils mit der Definition dessen zusammen, was genau von einer Generation an die nächste weitergegeben wird, und wie sich die weitergegebenen Ressourcen darauf auswirken, was aus den Kindern später einmal wird. Die Übertragungsmechanismen kommen im Allgemeinen über das (Finanz-, Human- und Sozial-) Kapital der Eltern sowie deren Intelligenz, Persönlichkeit, Lebensstil und Verhalten zum Tragen. Bei den beeinflussten Elementen handelt es sich um Haushaltseinkommen, Verdienst, Vermögen, Bildung, Beruf, usw.

Gestützt auf Schätzungen der intergenerativen Verdienstelastizität ist die Generationenmobilität in Dänemark, Norwegen, Finnland, Australien und Kanada am höchsten (dort liegt die Verdienstelastizität unter 0,2) und in Italien, den Vereinigten Staaten und dem Vereinigten Königreich am geringsten (mit Werten von rd. 0,5, vgl. Abb. EQ4.1). Die intergenerative Verdienstelastizität ist niedriger in Ländern mit größerer Einkommensungleichheit sowie (in geringerem Maße) in Ländern, in denen Bildung höhere wirtschaftliche Erträge bringt (Corak, 2006).

Bildung ist ein wichtiger Motor für die Generationenmobilität. In Tabelle EQ4.2 sind die Unterschiede zwischen den Mathematikergebnissen von 15-jährigen Schülerinnen und Schülern mit unterschiedlichem familiären Hintergrund dargestellt (an unterschiedlichen Punktzahlen können Bildungsunterschiede abgelesen werden; OECD, 2004). Unter den Faktoren, die sich auf die Kompetenzen der Schüler auswirken, scheint das Bildungsniveau der Eltern bei weitem am stärksten ins Gewicht zu fallen. Schülerinnen und Schüler, deren Eltern (Vater oder Mutter) ein niedrigeres Bildungsniveau haben, erzielen im Durchschnitt geringere Mathematikergebnisse als solche, deren Eltern ein sehr hohes Bildungsniveau aufweisen; in Klassenstufen ausgedrückt entspricht dieser Leistungsunterschied rd. 11/2 Jahren (in Ungarn, der Tschechischen Republik und der Slowakischen Republik mehr als 2 Jahren). Für Schülerinnen und Schüler, deren Eltern (Vater oder Mutter) ein mittleres Bildungsniveau haben, ist der Unterschied bei den Mathematikleistungen geringer (rd. $1 / 2$ Jahr), wobei es einige Ausnahmen gibt (z.B. Italien und Mexiko). Schülerinnen und Schüler aus Alleinerzieherhaushalten weisen ein geringeres Kompetenzniveau auf (der Unterschied entspricht bis zu über einer Klassenstufe in Belgien und den Vereinigten Staaten, während er in Österreich, der Tschechischen Republik und der Slowakischen Republik kaum wahrzunehmen ist). Auch Schüler, die in einem anderen Land als ihrem Geburtsland zur Schule gehen, und Schüler der zweiten Generation schneiden schlechter ab (der Leistungsunterschied gegenüber den Schülern ohne Migrationshintergrund entspricht im Durchschnitt über einer Klassenstufe). Schülerinnen und Schüler, in deren Elternhaus eine andere Sprache gesprochen wird, erzielen ebenfalls niedrigere Ergebnisse, besonders in Belgien und Deutschland. Der Leistungsunterschied zwischen Schülerinnen und Schülern, deren Eltern im untersten Quartil des PISA-Index des sozialen, wirtschaftlichen und kulturellen Status angesiedelt sind (dieser Index fasst die elterlichen Hintergrundmerkmale zusammen) und solchen, deren Eltern zum obersten Quartil gehören, entspricht im Schnitt 21/2 Klassenstufen (zwischen drei oder mehr Stufen in Ungarn sowie Belgien und weniger als zwei in Island, Finnland und Kanada).

Statusindikatoren: Materielle Entbehrung (EQ1), Beschäftigung (SS1), Schülerleistungen (SS7), Gesundheitliche Ungleichheit (HE6).

Reaktionsindikatoren: Öffentliche Sozialausgaben (EQ5). 


\section{EQ4.1 Geringere Generationenmobilität in Ländern mit größerer Einkommensungleichheit und höherer Bildungsrendite}

Intergenerative Einkommenselastizität, Einkommensungleichheit und Bildungsrendite in ausgewählten OECD-Ländern

Intergenerative Verdienstelastizität

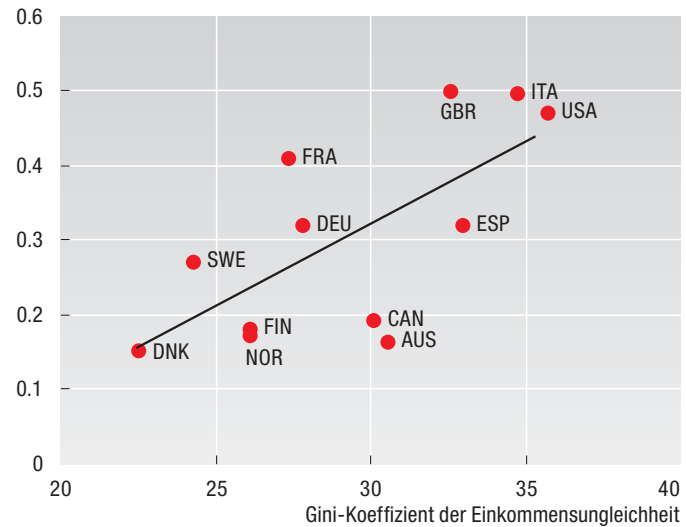

Intergenerative Verdienstelastizität

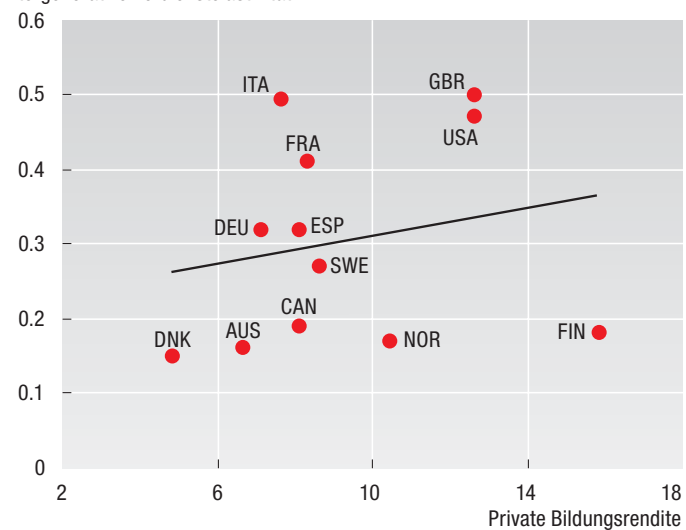

Quelle: Die Angaben zur Generationenmobilität stützen sich für die meisten Länder auf die Metaanalyse von Corak (2006). Die Angaben für Spanien, Australien und Italien stammen von D'Addio (2006). Die Daten zur privaten Bildungsrendite sind OECD, Bildung auf einen Blick, verschiedene Jahrgänge, entnommen. Die Angaben zum Gini-Koeffizienten der Einkommensungleichheit stammen aus früheren Ausgaben von Gesellschaft auf einen Blick - OECD-Sozialindikatoren.

EQ4.2 Schülerinnen und Schüler mit weniger gebildeten Eltern erzielen niedrigere Ergebnisse Punktzahlunterschiede im Vergleich zu andern Schülern

\begin{tabular}{|c|c|c|c|c|c|c|c|c|c|}
\hline & \multicolumn{2}{|c|}{$\begin{array}{c}\text { Bildungsstand } \\
\text { des Vaters: } \\
\text { Hoch im Vergleich zu: }\end{array}$} & \multicolumn{2}{|c|}{$\begin{array}{c}\text { Bildungsstand } \\
\text { der Mutter: } \\
\text { Hoch im Vergleich zu: }\end{array}$} & \multirow{2}{*}{$\begin{array}{c}\text { Paare } \\
\text { im Vergleich } \\
\text { zu: }\end{array}$} & \multicolumn{2}{|c|}{$\begin{array}{l}\text { Herkunftsland: Ohne } \\
\text { Migrationshintergrund } \\
\text { im Vergleich zu: }\end{array}$} & \multirow{2}{*}{$\begin{array}{c}\text { Sprache im Bternhaus: } \\
\text { Landessprache } \\
\text { im Vergleich zu: }\end{array}$} & \multirow{2}{*}{$\begin{array}{c}\begin{array}{c}\text { Wirtschaftlicher, sozialer und } \\
\text { kultureller Status (Index): } \\
\text { Oberstes Quartil im Vergleich zu }\end{array} \\
\text { Unterstes Quartil }\end{array}$} \\
\hline & Gering & Mittelhoch & Gering & Mittelhoch & & 2. Generation & $\begin{array}{l}\text { Im Ausland } \\
\text { Geborene }\end{array}$ & & \\
\hline Australien & -47 & -35 & -39 & -29 & -27 & -5 & -2 & -12 & -93 \\
\hline Belgien & -62 & -28 & -67 & -32 & -42 & -92 & -109 & -95 & -133 \\
\hline Dänemark & -63 & -41 & -61 & -25 & -26 & -70 & -65 & -43 & -101 \\
\hline Deutschland & -96 & -30 & -88 & -21 & -10 & -93 & -71 & -90 & -120 \\
\hline Finnland & -34 & -21 & -36 & -17 & -9 & . & . & . & -71 \\
\hline Frankreich & -50 & -19 & -55 & -17 & -18 & -48 & -72 & -66 & -105 \\
\hline Griechenland & -48 & -16 & -58 & -21 & -19 & $\ldots$ & -47 & -48 & -96 \\
\hline Irland & -49 & -24 & -49 & -19 & -33 & $\ldots$ & . & $\ldots$ & -86 \\
\hline Island & -38 & -20 & -38 & -22 & -8 & $\ldots$ & $\ldots$ & $\ldots$ & -61 \\
\hline Italien & -39 & 3 & -44 & -1 & -15 & . & . & $\ldots$ & -90 \\
\hline Japan & -66 & -34 & -57 & -28 & $\ldots$ & . & $\ldots$ & $\ldots$ & -88 \\
\hline Kanada & -41 & -23 & -45 & -21 & -20 & 6 & -7 & -13 & -74 \\
\hline Korea & -66 & -31 & -60 & -20 & -9 & . & . . & . & -90 \\
\hline Luxemburg & -61 & -24 & -53 & -25 & -19 & -31 & -45 & -42 & -102 \\
\hline Mexiko & -48 & 11 & -40 & 20 & -10 & $\ldots$ & $\ldots$ & $\ldots$ & -91 \\
\hline Neuseeland & -67 & -32 & -61 & -13 & -22 & -32 & -5 & -16 & -105 \\
\hline Niederlande & -46 & -29 & -40 & -33 & -31 & -59 & -79 & -81 & -99 \\
\hline Norwegen & -40 & -23 & -53 & -27 & -22 & . & -61 & -45 & -89 \\
\hline Österreich & -46 & -7 & -53 & -12 & -3 & -56 & -63 & -57 & -94 \\
\hline Polen & -86 & -55 & -95 & -54 & -13 & $\ldots$ & $\ldots$ & $\ldots$ & -95 \\
\hline Portugal & -31 & 11 & -41 & -2 & -10 & -30 & . & .. & -95 \\
\hline Schweden & -31 & -2 & -48 & -3 & -29 & -34 & -92 & -65 & -91 \\
\hline Schweiz & -60 & -9 & -56 & 2 & -16 & -59 & -89 & -79 & -103 \\
\hline Slowak. Rep. & -127 & -62 & -125 & -49 & -4 & . & .. & $\ldots$ & -116 \\
\hline Spanien & -47 & -27 & -43 & -25 & -12 & $\ldots$ & $\ldots$ & $\ldots$ & -85 \\
\hline Tschech. Rep. & -111 & -62 & -103 & -54 & -5 & . & . & $\ldots$ & -107 \\
\hline Türkei & -98 & -50 & -108 & -35 & -5 & $\ldots$ & $\ldots$ & $\ldots$ & -116 \\
\hline Ungarn & -120 & -64 & -115 & -58 & -16 & $\ldots$ & $\ldots$ & $\ldots$ & -127 \\
\hline Ver. Staaten & -74 & -35 & -76 & -29 & -43 & -22 & -36 & -46 & -109 \\
\hline OECD29 & -62 & -27 & -62 & -23 & -18 & -45 & -56 & -53 & -98 \\
\hline
\end{tabular}

Anmerkung: In jeder Spalte ist der Unterschied im Vergleich zu den durchschnittlichen Mathematikergebnissen der Schülerinnen und Schüler in den verschiedenen Ländern dargestellt. In der letzten Spalte steht der ungewichtete OECD-Durchschnitt, bei dessen Berechnung alle Länder gleich gewichtet wurden (in OECD 2004 waren dagegen gewichtete Durchschnitte angegeben).

Quelle: OECD (2004), Lernen für die Welt von Morgen: Erste Ergebnisse aus PISA 2003, Paris (w w w.pisa.oecd.org).

S tatLink 2 http://dx.doi.org/10.1787/618651183876

Weitere Informationen - Blanden, J., P. Gregg und S. Machin (2005), Intergenerational M obility in Europe and North A merica, Sutton Trust. - Corak, M. (2006), “Do Poor Children Become Poor Adults?”, IZA Discussion Paper, No. 1993. 口 D'Addio, A.C. (2006), “Mobility or Immobility across Generations? A review of the evidence for OECD countries", OECD Social, Employment and Migration Working Paper, erscheint demnächst, Paris. ¿ Solon, G. (2002), "Cross-Country Differences in Intergenerational Earnings Mobility", Journal of Economic Perspectives, Vol. 16, No. 3. 


\section{Definition und Messung}

Ein breites Spektrum von Personen und Institutionen (Verwandte und Freunde, öffentliche und private Einrichtungen) unterstützen auf verschiedenste Weise bedürftige Personen oder Haushalte. In entwickelten Marktwirtschaften wird ein Großteil dieser Unterstützung in Form von Sozialausgaben geleistet, bei denen es sich sowohl um finanzielle Beihilfen (durch Geldleistungen oder Steuervorteile) als auch um Sachleistungen in Form von Gütern und Dienstleistungen handeln kann. Um zu den Sozialausgaben gerechnet zu werden, müssen diese Leistungen auf eine oder mehrere Notlagen ausgerichtet sein, wie Einkommensschwäche, Alter, Arbeitslosigkeit oder Behinderung. Programme zur Bereitstellung von Sozialleistungen beruhen entweder auf einer Umverteilung von Ressourcen zwischen den privaten Haushalten oder auf dem Prinzip der Pflichtmitgliedschaft.

Sozialausgaben gelten als öffentlich, wenn die entsprechenden Finanzströme vom Staat (d.h. von einer Zentralregierung, den nachgeordneten Gebietskörperschaften oder den Sozialversicherungsträgern) kontrolliert werden. Krankengeld ist beispielsweise eine "öffentliche" Sozialleistung, wenn es aus Pflichtbeiträgen der Arbeitgeber und Arbeitnehmer zur Sozialversicherung finanziert wird, und eine "private" Leistung, wenn es direkt vom Arbeitgeber an den Arbeitnehmer gezahlt wird. Der für die Zwecke des Ländervergleichs am häufigsten verwendete Indikator für die Sozialausgaben bezieht sich auf die öffentlichen Ausgaben im Verhältnis zum BIP in Marktpreisen. Die hier dargestellten Ausgabenströme sind "brutto" angegeben, d.h. vor Abzug von direkten und indirekten, auf diese Leistungen zu entrichtenden Steuern und vor Addition von Steuererleichterungen für soziale Zwecke. Messprobleme existieren insbesondere im Hinblick auf die Ausgaben der nachgeordneten Gebietskörperschaften, die in einigen Ländern möglicherweise unterschätzt werden.

2003 machten die öffentlichen Bruttosozialausgaben im OECD-Durchschnitt 21\% des BIP aus (Abb. EQ5.1), wobei die Geldleistungen doppelt so hoch waren wie die Sachleistungen. Bei der Höhe der Ausgaben bestehen große Unterschiede zwischen den Ländern, sie reichen von $6 \%$ des BIP in Mexiko und Korea bis über $30 \%$ in Schweden.

Die drei größten Kategorien von öffentlichen Sozialausgaben sind die Renten (Alters- und Hinterbliebenenrenten, durchschnittlich 7\% des BIP), die Gesundheitsausgaben (6\%) und die Einkommenstransfers für Personen im Erwerbsalter (5\%). Innerhalb der dritten Kategorie belaufen sich die öffentlichen Ausgaben für Haushalte mit Kindern und Personen mit Behinderungen jeweils auf fast $2 \%$ des BIP. In Frankreich, Griechenland, Italien, Österreich und Polen betragen die Ausgaben für Alters- und Hinterbliebenenrenten mehr als $12 \%$ des BIP, in Australien, Irland, Island, Korea, Mexiko und der Türkei weniger als $4 \%$. Auf mehr als $5 \%$ des BIP belaufen sich die öffentlichen Bruttoausgaben für soziale Leistungen nur in den nordischen Ländern, wo dem Staat eine umfassende Funktion bei der Bereitstellung von Leistungen für Ältere, Menschen mit Behinderungen und Familien zukommt.

Auch im Zeitverlauf sind erhebliche Veränderungen bei den öffentlichen Bruttosozialausgaben im Verhältnis zum BIP festzustellen (Abb. EQ5.2). Seit 1980 sind die öffentlichen Bruttosozialausgaben im Durchschnitt von 27 OECDLändern von rd. 16\% auf 21\% des BIP im Jahr 2003 gestiegen. Die Entwicklung verlief in den einzelnen OECD-Ländern unterschiedlich, am stärksten wuchsen die öffentlichen
Bruttosozialausgaben im Verhältnis zum BIP durchschnittlich jedoch Anfang der achtziger Jahre, Anfang der neunziger Jahre und nochmals Anfang dieses Jahrzehnts, als sich das Verhältnis durchschnittliche öffentliche Sozialausgaben/BIP zwischen 2000 und 2003 um 1\% des BIP erhöhte. Zwischen den drei Jahrzehntwenden veränderte sich das Verhältnis öffentliche Sozialausgaben/BIP kaum; während der achtziger Jahre bewegte es sich im OECD-Durchschnitt knapp unter $20 \%$ des BIP, wohingegen es in den neunziger Jahren nach dem Abschwung zu Beginn des Jahrzehnts tendenziell sank, aber dennoch über $20 \%$ des BIP verharrte. In den meisten OECD-Ländern lagen die öffentlichen Sozialausgaben im Verhältnis zum BIP 2003 weit über ihrem Niveau der achtziger Jahre, außer in den Benelux-Staaten und insbesondere in den Niederlanden, wo andauerndes Wirtschaftswachstum, die Verschärfung der Anspruchskriterien und Kürzung der Leistungshöhe der Invaliditätsversicherung wie auch die Privatisierung des Krankengelds in den neunziger Jahren zu einem Rückgang der öffentlichen Sozialausgaben im Verhältnis zum BIP um 4\% führten.

Statusindikatoren: Materielle Entbehrung (EQ1), Armutspersistenz (EQ7), Arbeitslosigkeit (SS2).

Reaktionsindikatoren: Gesamtsozialausgaben (EQ6), Leistungen bei Erwerbslosigkeit (SS6), Gesundheitsausgaben (HE2). 


\section{EQ5.1 Die öffentlichen Sozialausgaben machen im OECD-Durchschnitt ein Fünftel des BIP aus} Öffentliche Bruttosozialausgaben nach Kategorie, in Prozent des BIP, $2003^{1}$

Geldleistungen

Einkommenstransfer für Personen im Erwerbsalter

Renten (Alters- und Hinterbliebenenrenten)

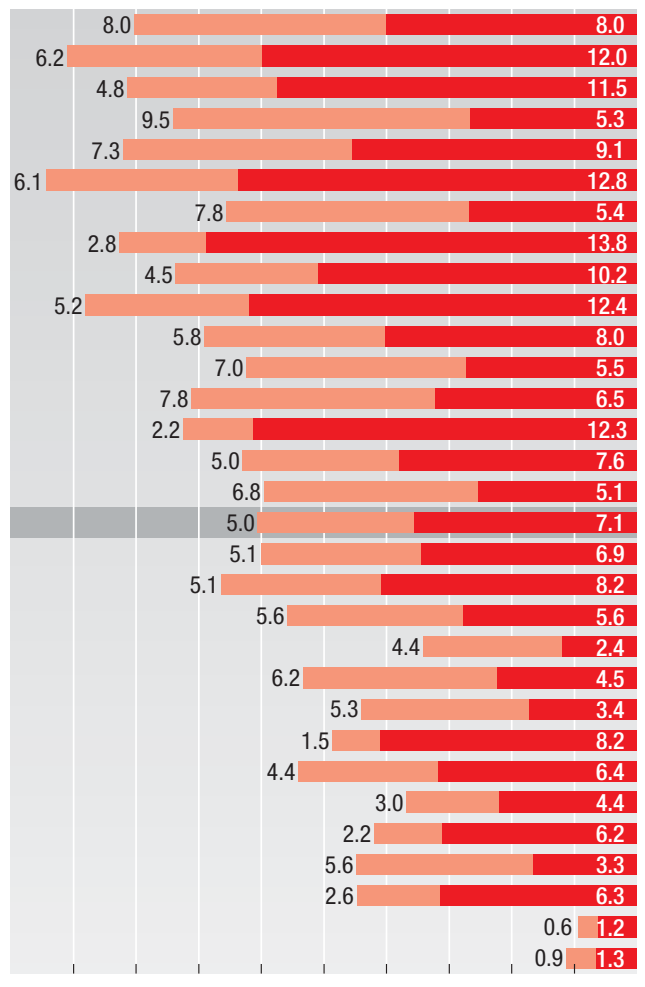

Sachleistungen

Alle Sozialdienste (ohne Gesundheit)

Gesundheitsausgaben

Schweden (31.3)

Frankreich (28.7)

Deutschland (27.6)

Dänemark (27.6)

Belgien (26.5)

Österreich (26.1)

Norwegen (25.1)

Italien (24.2)

Portugal (23.5)

Polen (22.9)

Ungarn (22.7)

Finnland (22.5)

Luxemburg (22.2)

Griechenland (21.3)

Tschech. Rep. (21.1)

Niederlande (20.7)

OECD30 (20.7)

Schweiz (20.5)

Spanien (20.3)

Ver. Königreich (20.1)

Island (18.7)

Neuseeland (18)

Australien (17.9)

Japan (17.7)

Slowak Rep. (17.3)

Kanada (17.3)

Ver. Staaten (16.2)

Irland (15.9)

Türkei (13.2)

Mexiko (6.8)

Korea (5.7)

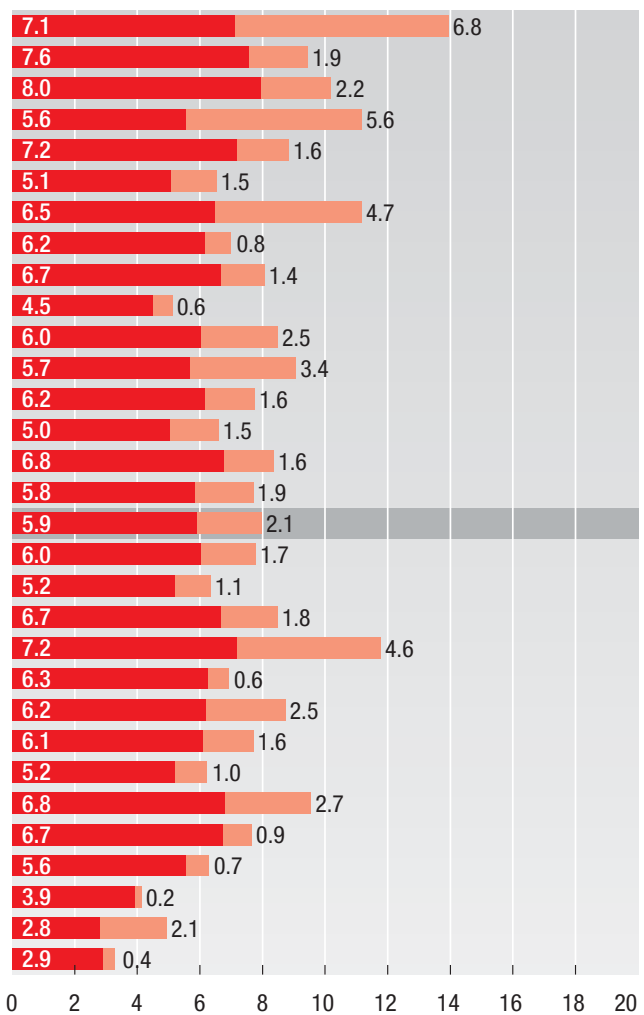

1. 1999 statt 2003 für die Türkei.

Anmerkung: Die Länder sind in absteigender Reihenfolge nach der Höhe der öffentlichen Sozialausgaben im Verhältnis zum BIP dargestellt. Ausgaben für aktive Arbeitsmarktprogramme (AAMP) können nicht in Bar- und Sachleistungen aufgeschlüsselt werden; in den öffentlichen Gesamtausgaben sind sie allerdings berücksichtigt (Werte in Klammern).

EQ5.2 Die öffentlichen Sozialausgaben sind im Verhältnis zum BIP seit 2000 wieder gestiegen Öffentliche Bruttosozialausgaben in ausgewählten OECD-Ländern, in Prozent des BIP, 1980-2003
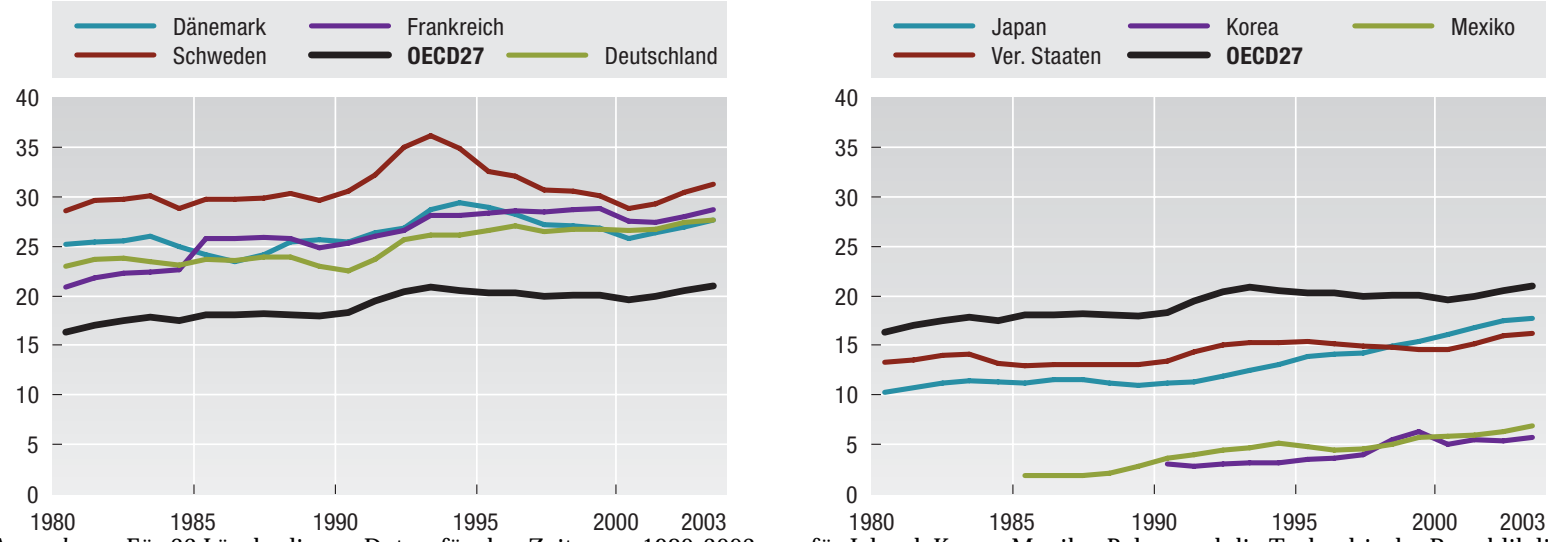

Anmerkung: Für 22 Länder liegen Daten für den Zeitraum 1980-2003 vor, für Island, Korea, Mexiko, Polen und die Tschechische Republik liegen jedoch nur Daten ab 1990 vor. OECD27 bezieht sich auf einen ungewichteten Durchschnittswert der OECD-Länder ohne Ungarn (Daten ab 1999), Slowakische Republik (Daten ab 1995) und Türkei (keine Daten ab 2000).

Quelle: OECD (2006), Social Expenditure Database 1980-2003, Paris (www.oecd.org/els/social/expenditure).

St at Link 2

http://dx.doi.org/10.1787/634461464361

Weitere Informationen — Adema, W. und M. Ladaique (2005), “Net Social Expenditure, 2005 Edition - More comprehensive measures of social support", OECD Social, Employment and Migration Working Paper, No. 29, Paris (www.oecd.org/els/workingpapers). 


\section{Definition und Messung}

Für eine umfassende Beurteilung der Gesamthöhe der Ressourcen, die von den OECD-Ländern für soziale Zwecke aufgewendet werden, gilt es sowohl die öffentlichen als auch die privaten Sozialausgaben zu berücksichtigen und zu untersuchen, inwieweit sich das Steuersystem auf die tatsächliche Höhe der geleisteten Unterstützung auswirkt. Zur Erfassung des Effekts des Steuersystems auf die "Bruttosozialausgaben", d.h. die Ausgaben vor Steuern, muss ermittelt werden, welcher Teil der Sozialausgaben durch die direkte Besteuerung der Transfereinkommen und die indirekte Besteuerung der von den Leistungsempfängern konsumierten Güter und Dienstleistungen wieder an den Staat zurückfließt. Zudem kann der Staat soziale Ziele auch dadurch fördern, dass er sozial motivierte Steuervorteile gewährt (z.B. Steuerfreibeträge für Kinder). Aus Sicht der Gesellschaft sind die "Nettosozialausgaben" (d.h. nach Steuern) aus öffentlichen und privaten Quellen ein besserer Indikator für den Umfang der für soziale Zwecke aufgewendeten Ressourcen. Aus Sicht des Einzelnen spiegelt sich in den "Nettosozialausgaben" der Teil der gesamtwirtschaftlichen Produktion wider, der von Leistungsempfängern in Anspruch genommen wird.

Zur Messung des Effekts des Steuersystems auf die Sozialausgaben sind häufig Schätzungen auf der Grundlage von Mikrodaten und Mikrosimulationsmodellen erforderlich, weil es oft an entsprechenden Verwaltungsdaten fehlt. Zudem liegen nicht immer zentral erfasste Daten zu den privaten Sozialausgaben vor. Daher sind die entsprechenden Informationen von geringerer Qualität als die Daten zu den öffentlichen Bruttosozialausgaben. Da eine Berichtigung um indirekte Steuern vorgenommen werden muss, werden die Nettosozialausgaben im Verhältnis zum BIP zu Faktorkosten und nicht zu Marktpreisen gemessen.

In Tabelle EQ6.1 ist der Effekt der Steuerzahlungen und Steuervergünstigungen auf die staatlichen Bruttosozialausgaben in ausgewählten OECD-Ländern im Jahr 2003 dargestellt. Dabei fallen drei Dinge auf. Erstens ist der "Rückfluss" der Bruttosozialausgaben in Form direkter, auf Transfereinkommen zu entrichtender Steuern in Dänemark und Schweden am höchsten, wo fast 15\% der Geldleistungen über Einkommensteuern und Sozialversicherungsbeiträge wieder in die staatlichen Kassen zurückfließen. Zweitens ist der Teil der Bruttosozialausgaben, der in Form indirekter Steuern zurückfließt, in den europäischen OECD-Ländern höher als in den nichteuropäischen. Drittens machen Länder, in denen nur wenig direkte Steuern auf Transferleistungen erhoben werden - z.B. Kanada, Deutschland und die Vereinigten Staaten -, häufiger von Steuervergünstigungen für Empfänger von Nichtrentenleistungen Gebrauch. Auf Grund von Lücken in den Datenreihen und konzeptueller Probleme bei ihrer Messung sind Steuervergünstigungen für Altersrentenempfänger - die es nur in ein paar Ländern gibt - in Tabelle EQ6.1 gesondert ausgewiesen.

Im Allgemeinen holt sich der Staat über die Besteuerung öffentlicher Sozialleistungen mehr zurück, als er in Form von sozial motivierten Steuervorteilen ausgibt. Folglich waren die öffentlichen Nettosozialausgaben 2003 in den 24 in Tabelle EQ6.1 dargestellten Ländern rd. 3 Prozentpunkte geringer als die entsprechenden Bruttoausgaben. Die einzigen Ausnahmen bildeten Mexiko und die Vereinigten Staaten (wo die öffentlichen Sozialausgaben netto höher waren als brutto) und Korea (wo die beiden Ausgabenaggregate in etwa gleich hoch waren).

Im Durchschnitt einer Gruppe von 24 OECD-Ländern machten die Nettosozialausgaben 2003 etwas mehr als 23\% des BIP aus, wobei dieser Prozentsatz von über $30 \%$ in Deutschland, Frankreich und Schweden bis zu weniger als $10 \%$ in Korea und Mexiko reichte. Bei Berücksichtigung sowohl der privaten Sozialleistungen als auch des Effekts des Steuersystems verringern sich die zwischen den Ländern zu beobachtenden Unterschiede bei der Höhe der Sozialausgaben im Verhältnis zum BIP. Der an den Nettogesamtsozialausgaben gemessene Teil der gesamtwirtschaftlichen Produktion, der von Sozialleistungsempfängern in Anspruch genommen wird, ist in Dänemark, den Niederlanden, Norwegen, Österreich, dem Vereinigten Königreich und den Vereinigten Staaten de facto ziemlich identisch (Abb. EQ6.2). Ein im Ländervergleich ähnlicher Umfang der Nettosozialausgaben bedeutet jedoch nicht, dass der Grad der vom Steuer- und Transfersystem gewährleisteten Umverteilung ebenfalls identisch oder dass der Effekt auf die Volkswirtschaft gleich wäre.

Statusindikatoren: Materielle Entbehrung (EQ1), Armutspersistenz (EQ7), Arbeitslosigkeit (SS2).

Reaktionsindikatoren: Öffentliche Sozialausgaben (EQ5), Leistungen bei Erwerbslosigkeit (SS6), Gesundheitsausgaben (HE2). 


\section{EQ6.1 Öffentliche Brutto- und Nettosozialausgaben}

In Prozent des BIP zu Faktorkosten, 2003

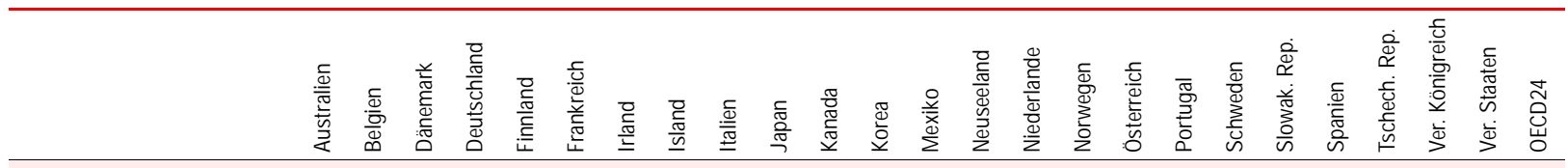

\section{Öfentliche}

Bruttosozialausgaben

- Auf Transfereinkommen zu entrichtende direkte

Stevern und Sozial-

versicherungsbeiträge

- Indirekte Besteuerung der

von Leistungsempfängern

konsumierten Güter

und Dienstleistungen

+ Steuervergünstigungen

für Empfänger von

Transferleistungen

(ohne Renten)

= Öfentliche

Nettosozialausgaben

Nachrichtlich

Steuervergünstigungen

für Rentner

.: Daten nicht verfügbar

- Null.

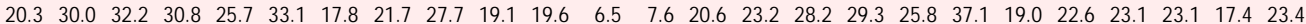

$\begin{array}{lllllllllllllllllllllllll}0.2 & 1.8 & 4.8 & 1.3 & 2.5 & 1.5 & 0.3 & 0.8 & 2.0 & 0.3 & 0.4 & 0.0 & - & 1.6 & 1.8 & 2.4 & 2.9 & 0.7 & 5.0 & - & 1.3 & 0.0 & 0.2 & 0.7 & 1.5\end{array}$

$\begin{array}{lllllllllllllllllllllllll}1.0 & 2.7 & 3.9 & 2.5 & 3.0 & 3.3 & 2.4 & 1.7 & 2.4 & 0.7 & 1.0 & 0.4 & 0.2 & 2.0 & 2.2 & 3.2 & 3.2 & 3.2 & 3.4 & 2.0 & 2.1 & 2.3 & 2.0 & 0.4 & 2.1\end{array}$

$\begin{array}{lllllllllllllllllllllllll}0.2 & 0.5 & 0.0 & 2.1 & - & 1.0 & 0.4 & - & 0.3 & 0.8 & 1.3 & 0.6 & 1.0 & 0.1 & 0.8 & 0.1 & 0.0 & 1.0 & - & 0.6 & 0.4 & 0.5 & 0.5 & 2.3 & 0.7\end{array}$

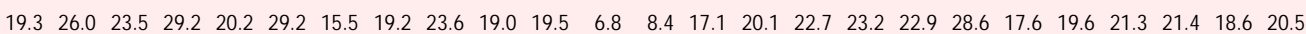

$\begin{array}{llllllllllllllllllllllllll}2.0 & 0.2 & \ldots & 0.9 & 0.2 & 0.0 & 2.2 & 1.2 & 0.0 & 0.7 & 1.9 & \ldots & 0.1 & \ldots & \ldots & 0.8 & 0.1 & 0.1 & 0.0 & 0.0 & 0.3 & 0.1 & 1.1 & 1.3 & \ldots\end{array}$

\section{EQ6.2 Öffentliche Sozialausgaben und Gesamtsozialausgaben}

In Prozent des BIP zu Faktorkosten, 2003

A. Öffentliche Sozialausgaben

Bruttoausgaben

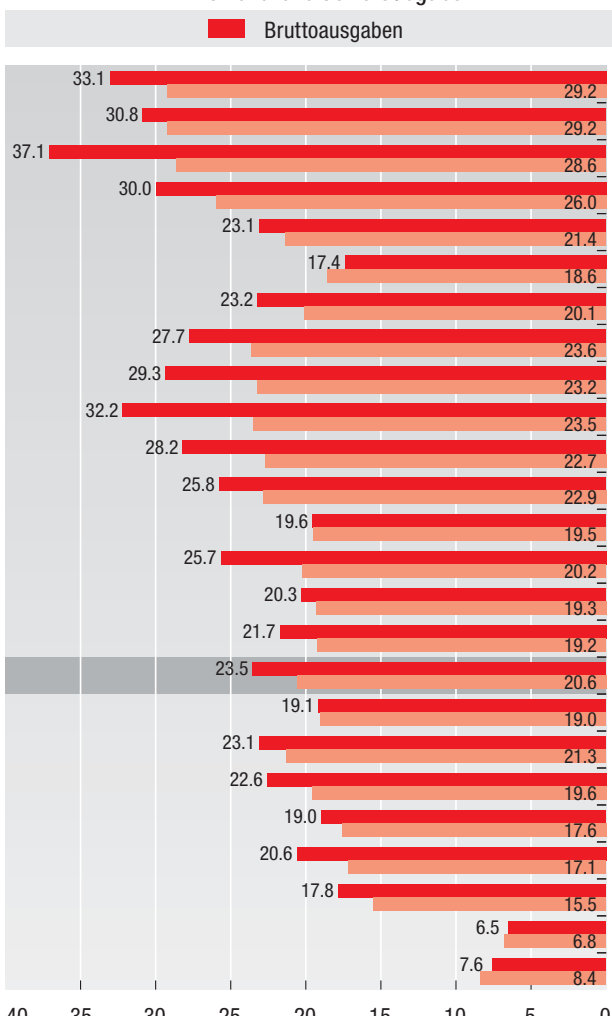

B. Gesamte (öffentliche + private) Sozialausgaben Nettoausgaben

$$
\begin{aligned}
& \text { Frankreich } \\
& \text { Deutschland } \\
& \text { Schweden } \\
& \text { Belgien } \\
& \text { Ver. Königreich } \\
& \text { Ver. Staaten } \\
& \text { Niederlande } \\
& \text { Italien } \\
& \text { Österreich } \\
& \text { Dänemark } \\
& \text { Norwegen } \\
& \text { Portugal } \\
& \text { Kanada } \\
& \text { Finnland } \\
& \text { Australien } \\
& \text { Island } \\
& \text { 0ECD24 } \\
& \text { Japan } \\
& \text { Tschech. Rep. } \\
& \text { Spanien } \\
& \text { Slowak. Rep. } \\
& \text { Neuseeland } \\
& \text { Irland } \\
& \text { Korea } \\
& \text { Mexiko }
\end{aligned}
$$

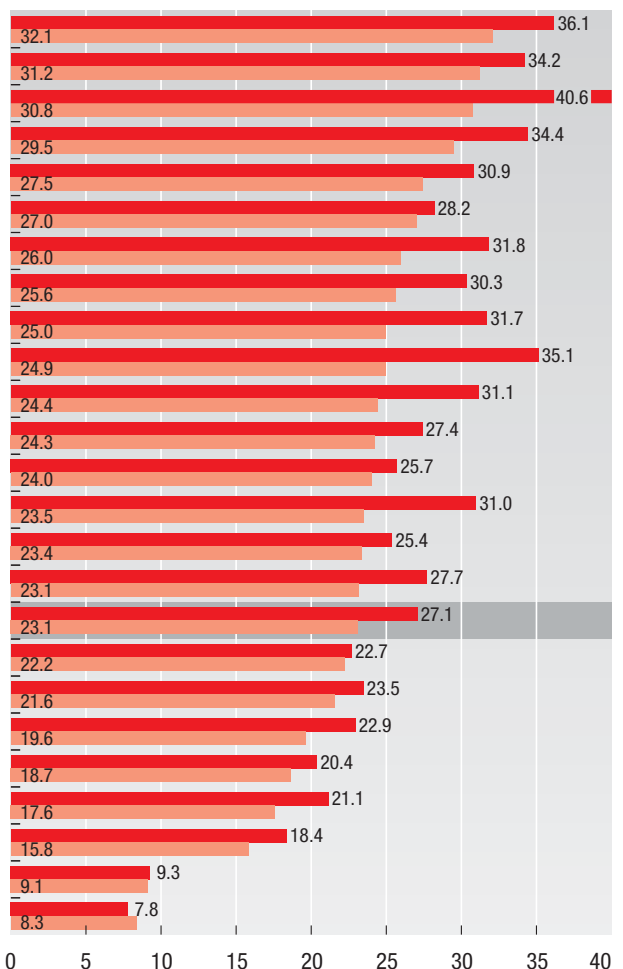

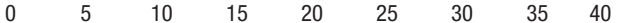

Anmerkung: Die Länder sind in absteigender Reihenfolge nach der Höhe der gesamten Nettosozialausgaben dargestellt; da eine Berichtigungum indirekte Steuern vorgenommen werden muss, werden die Nettosozialausgaben im Verhältnis zum BIP zu Faktorkosten und nicht zu Marktpreisen gemessen. Quelle: OECD (2006), Social Expenditure Database 1980-2003, Paris (ww w.oecd.org/els/social/expenditure).

S tatLink $2 \quad$ http://dx.doi.org/10.1787/535736620712

Weitere Informationen — Adema, W. (2001), “Net Social Expenditure, 2nd Edition”, OECD Labour Market and Social Policy Occasional Paper, No. 52, Paris (ww w.oecd.org/els/w orkingpapers). I OECD (2006), OECD Social Expenditure Database, 1980-2003, Paris (ww w.oecd.org/els/ social/expenditure/SOCX). Adema, W. und M. Ladaique (2005), "Net Social Expenditure, 2005 Edition - More comprehensive measures of social support", OECD Social, Employment and Migration Working Paper, No. 29, Paris (ww w.oecd.org/els/w orkingpapers). 


\section{Definition und Messung}

Die Armutspersistenz kann an der Zahl der Personen gemessen werden, deren Einkommen während eines Zeitraums von drei Jahren unter einer bestimmten Schwelle liegt (die im Allgemeinen im Verhältnis zum mittleren verfügbaren Einkommen festgelegt wird). Diese Messgröße wird anhand von Aufstellungen aus Erhebungen berechnet, in denen Einzelpersonen im Zeitverlauf beobachtet werden. Es gibt mehrere mögliche Definitionen anhaltender Armut. Eine besteht darin, den Anteil der Personen zu messen, die während des Dreijahreszeitraums "ständig arm" (d.h. die "anhaltende Armut") sind. Bei anderen Definitionen werden auch Personen berücksichtigt, die in zwei dieser drei Jahre arm sind ("wiederkehrende Armut"), oder Personen, die in diesem Zeitraum nur einmal arm sind ("einmalige Armut"). Als Einkommenskonzept wird dabei das verfügbare Jahreseinkommen (d.h. nach Transferleistungen und Zahlung von Einkommensteuer und Sozialversicherungsbeiträgen) der privaten Haushalte verwendet, wobei jedem Haushaltsmitglied das “äquivalisierte” Einkommen seines Haushalts zugewiesen wird, gestützt auf einen allgemein üblichen Faktor zur Berichtigung um unterschiedliche Haushaltsgrößen (Quadratwurzelelastizität).

Die Angaben beziehen sich für die europäischen Länder auf den Zeitraum 1999-2001 und stützen sich auf das Haushaltspanel der Europäischen Gemeinschaften (ECHP), für die Vereinigten Staaten auf den Zeitraum 2001-2003 (Survey of Income and Program Participation - SIPP), für Kanada auf die internationale Äquivalenzdatei für den Zeitraum 2001-2003 (Survey of Labour and Income Dynamics - SLID) und für Australien auf den Zeitraum 2002-2004 (Household Income and Labour Dynamic in Australia - HILDA).

Mit der am häufigsten verwendeten Messgröße der relativen Einkommensarmut (der Headcount Ratio bzw. Armutsquote, die zumeist ausgehend von Daten ermittelt wird, die nur eine Momentaufnahme der Situation zum jeweiligen Zeitpunkt liefern) kann nicht gemessen werden, ob die Einkommensschwäche dauerhaft oder vorübergehend ist. Die meisten Menschen können mit einer kurzen Phase der Einkommensschwäche fertig werden, dauert diese Situation jedoch länger an, kann sie zu materieller Not und Ausgrenzung führen. In Tabelle EQ7.1 ist - gestützt auf Erhebungen, in denen Einzelpersonen oder Haushalte über einen längeren Zeitraum beobachtet wurden - die Relation zwischen verschiedenen Armutsmaßen aufgezeigt.

Bei einer an der Hälfte des Medianeinkommens orientierten Armutsschwelle lagen die "durchschnittlichen" Armutsquoten in einem Dreijahreszeitraum in den 17 in Tabelle EQ7.1 berücksichtigten Ländern bei rd. 10\%, wobei sie zwischen $6 \%$ oder weniger in den Niederlanden, Dänemark sowie Deutschland und $14 \%$ oder mehr in Australien, Irland, den Vereinigten Staaten und Griechenland schwankten. Bei einer Armutsschwelle von $40 \%$ des Medianeinkommens sinkt die Armutsquote im OECD-Durchschnitt auf $5 \%$, wohingegen sie bei einer mit $60 \%$ des Medianeinkommens angesetzten Schwelle 16\% übersteigt. Bei diesem Armutsmaß wird die Rangfolge der Länder kaum von der Definition der Armutsschwelle beeinflusst, d.h. die Länder, in denen die Armutsquote bei einer bestimmten Armutsschwelle hoch ist, liegen auch bei anders angesetzten Schwellenwerten im oberen Bereich.

Diese Headcount Ratio liefert jedoch nur ein unvollständiges Bild der Armutssituation, da viele Menschen vorübergehende Phasen der Armut erleben, während sich andere über einen längeren Zeitraum oder über viele Jahre hinweg wiederholt mit Armut konfrontiert sehen (Burkhauser, 2001; OECD, 2006). Bei einer mit der Hälfte des Medianeinkommens angesetzten Armutsschwelle - was in den OECD-
Analysen am üblichsten ist - waren durchschnittlich rd. $83 \%$ aller Befragten in den drei betrachteten Jahren niemals arm, was heißt, dass $17 \%$ in diesem Zeitraum zumindest einmal Armut erlebten. In dieser Gruppe waren diejenigen, die in den drei Jahren nur einmal arm waren, am zahlreichsten (7,7\%), während rd. 5\% der Erhebungspopulation während dieses Zeitraums durchgehend arm waren. Ein etwas geringerer Teil (durchschnittlich 4\%) war in zwei der drei beobachteten Jahre arm. Die Unterschiede zwischen den Ländern in Bezug auf die Verbreitung anhaltender Armut sind ebenso groß wie bei den durchschnittlichen Armutsquoten. Wie aus Abbildung EQ7.2 ersichtlich ist, verzeichnen Länder, in denen die einfache Armutsquote höher ist, auch eine größere Prävalenz sowohl anhaltender als auch wiederkehrender Armut.

Für Kinder und ältere Menschen ist offenbar nicht nur das an der Armutsquote gemessene Risiko, in einem gegebenen Jahr arm zu sein, höher als unter der Bevölkerung im Erwerbsalter, sondern auch die Wahrscheinlichkeit, über einen längeren Zeitraum hinweg arm zu bleiben. Bei einer mit der Hälfte des Medianeinkommens angesetzten Armutsschwelle stellen die "anhaltend Armen" im Durchschnitt rd. 40\% der 25- bis 64-Jährigen, die zu einem gegebenen Zeitpunkt arm sind, wohingegen sie unter den Kindern fast 50\% ausmachen und unter den Älteren über $60 \%$. Auch allein lebende Frauen sind mit einem Anteil der "anhaltend Armen", der in etwa der Hälfte der Länder $10 \%$ übersteigt, einem höheren Risiko persistenter Armut ausgesetzt.

Statusindikatoren: Materielle Entbehrung (EQ1).

Reaktionsindikatoren: Öffentliche Sozialausgaben (EQ5), Leistungen bei Erwerbslosigkeit (SS6). 


\section{EQ7.1 Fast 5\% der Befragten waren während eines Dreijahreszeitraums anhaltend arm}

Dauer der Armutsphase in einem Dreijahreszeitraum in ausgewählten OECD-Ländern

\begin{tabular}{|c|c|c|c|c|c|c|c|c|c|c|c|c|}
\hline & \multicolumn{12}{|c|}{ Dauer der Armutsphase in einem Dreijahreszeitraum, bei unterschiedlich angesetzten Armutsschwellen } \\
\hline & \multicolumn{4}{|c|}{$40 \%$ des Medianeinkommens } & \multicolumn{4}{|c|}{$50 \%$ des Medianeinkommens } & \multicolumn{4}{|c|}{$60 \%$ des Medianeinkommens } \\
\hline & $\begin{array}{l}\text { Durch- } \\
\text { schnittliche } \\
\text { Armutsraten } \\
\text { in den } \\
\text { 3 Jahren }\end{array}$ & $\begin{array}{l}\text { Enmalige } \\
\text { Armut }\end{array}$ & $\begin{array}{c}\text { Wieder- } \\
\text { kehrende } \\
\text { Armut }\end{array}$ & $\begin{array}{c}\text { Anhaltende } \\
\text { Armut }\end{array}$ & $\begin{array}{c}\text { Durch- } \\
\text { schnittliche } \\
\text { Armutsraten } \\
\text { in den } \\
\text { 3 Jahren }\end{array}$ & $\begin{array}{c}\text { Enmalige } \\
\text { Armut }\end{array}$ & $\begin{array}{l}\text { Wieder- } \\
\text { kehrende } \\
\text { Armut }\end{array}$ & $\begin{array}{c}\text { Anhaltende } \\
\text { Armut }\end{array}$ & $\begin{array}{c}\text { Durch- } \\
\text { schnittliche } \\
\text { Armutsraten } \\
\text { in den } \\
\text { 3 Jahren }\end{array}$ & $\begin{array}{c}\text { Enmalige } \\
\text { Armut }\end{array}$ & $\begin{array}{l}\text { Wieder- } \\
\text { kehrende } \\
\text { Armut }\end{array}$ & $\begin{array}{c}\text { Anhaltende } \\
\text { Armut }\end{array}$ \\
\hline Australien & 6.2 & 9.0 & 2.5 & 1.6 & 14.0 & 10.5 & 6.5 & 6.5 & 20.8 & 11.5 & 8.1 & 11.8 \\
\hline Belgien & 3.0 & 4.5 & 1.2 & 0.7 & 6.7 & 7.2 & 2.4 & 2.6 & 13.7 & 10.3 & 5.4 & 6.5 \\
\hline Dänemark & 2.4 & 4.4 & 0.9 & 0.2 & 5.7 & 7.3 & 2.1 & 1.7 & 11.0 & 9.4 & 4.2 & 4.8 \\
\hline Deutschland & 3.3 & 3.8 & 1.5 & 0.9 & 6.1 & 5.6 & 2.9 & 2.3 & 11.0 & 7.2 & 4.3 & 5.7 \\
\hline Finnland & 2.9 & 3.2 & 1.1 & 0.9 & 6.8 & 5.9 & 2.5 & 2.8 & 12.9 & 5.9 & 5.9 & 6.5 \\
\hline Frankreich & 3.6 & 4.7 & 1.9 & 0.7 & 7.9 & 7.6 & 3.6 & 2.8 & 14.9 & 9.4 & 6.4 & 7.2 \\
\hline Griechenland & 9.1 & 7.8 & 4.5 & 3.5 & 14.6 & 9.7 & 6.5 & 7.1 & 21.4 & 11.6 & 8.2 & 12.1 \\
\hline Irland & 6.9 & 7.3 & 3.6 & 1.9 & 15.0 & 7.9 & 6.3 & 8.1 & 22.2 & 9.6 & 9.4 & 12.6 \\
\hline Italien & 7.9 & 7.2 & 4.3 & 2.6 & 12.6 & 8.3 & 6.1 & 5.6 & 19.6 & 10.1 & 8.5 & 10.3 \\
\hline Kanada & 6.9 & 5.9 & 3.4 & 3.0 & 11.8 & 7.4 & 4.8 & 6.6 & 18.7 & 8.3 & 6.9 & 11.8 \\
\hline Luxemburg & 1.7 & 3.2 & 0.8 & 0.2 & 5.9 & 4.4 & 3.0 & 2.6 & 13.0 & 6.0 & 5.0 & 7.9 \\
\hline Niederlande & 2.9 & 4.8 & 1.5 & 0.4 & 5.2 & 5.7 & 3.2 & 1.3 & 9.6 & 8.4 & 5.1 & 3.7 \\
\hline Österreich & 3.6 & 4.7 & 1.3 & 1.2 & 7.1 & 6.7 & 2.8 & 2.9 & 13.0 & 9.1 & 4.8 & 6.6 \\
\hline Portugal & 7.6 & 6.9 & 2.6 & 3.5 & 13.5 & 8.4 & 5.1 & 7.1 & 20.4 & 10.8 & 6.5 & 12.3 \\
\hline Spanien & 7.3 & 9.8 & 3.1 & 1.9 & 13.9 & 11.1 & 6.8 & 5.5 & 20.9 & 12.6 & 8.8 & 10.4 \\
\hline Ver. Königreich & 6.5 & 8.4 & 3.0 & 1.6 & 11.9 & 9.4 & 5.3 & 5.1 & 19.2 & 10.9 & 7.9 & 10.1 \\
\hline Ver. Staaten & 8.7 & 6.5 & 3.5 & 3.6 & 13.9 & 8.6 & 5.6 & 7.2 & 20.9 & 10.6 & 7.3 & 12.4 \\
\hline OECD17 & 5.3 & 6.0 & 2.4 & 1.7 & 10.0 & 7.7 & 4.4 & 4.6 & 16.8 & 9.5 & 6.6 & 9.0 \\
\hline
\end{tabular}

Anmerkung: Die Daten beziehen sich auf drei Jahre Anfang dieses Jahrzehnts. Relative Einkommensarmut gemäß dem äquivalisierten verfügbaren Haushaltseinkommen. Alle Maße gründen sich auf eine Stichprobe von Personen, die während des jeweiligen Dreijahreszeitraums beobachtet wurden.

\section{EQ7.2 Ständige und wiederkehrende Armut sind in Ländern mit höheren Armutsquoten weiter verbreitet} Bei 50\% des Medianeinkommens angesetzte Armutsschw elle

Durchschnittliche Armutsraten

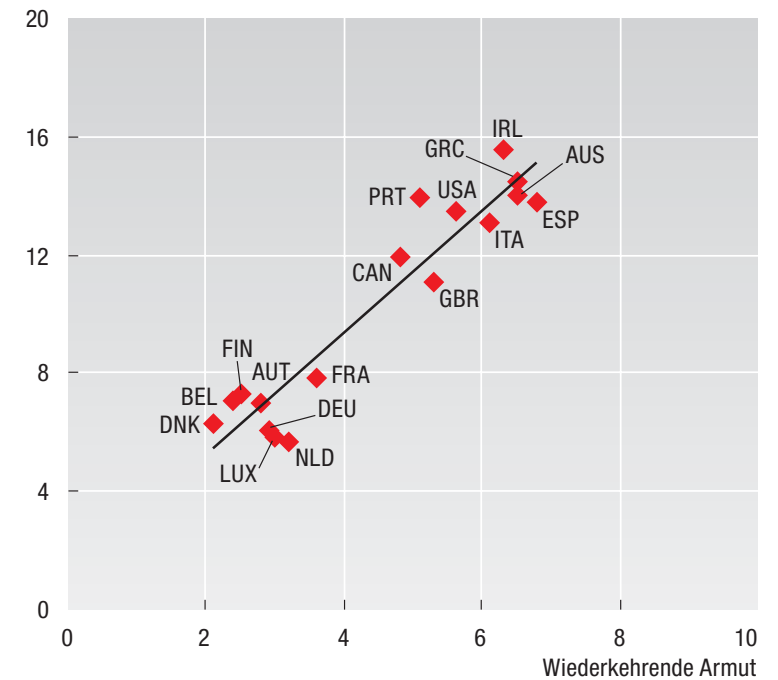

Durchschnittliche Armutsraten

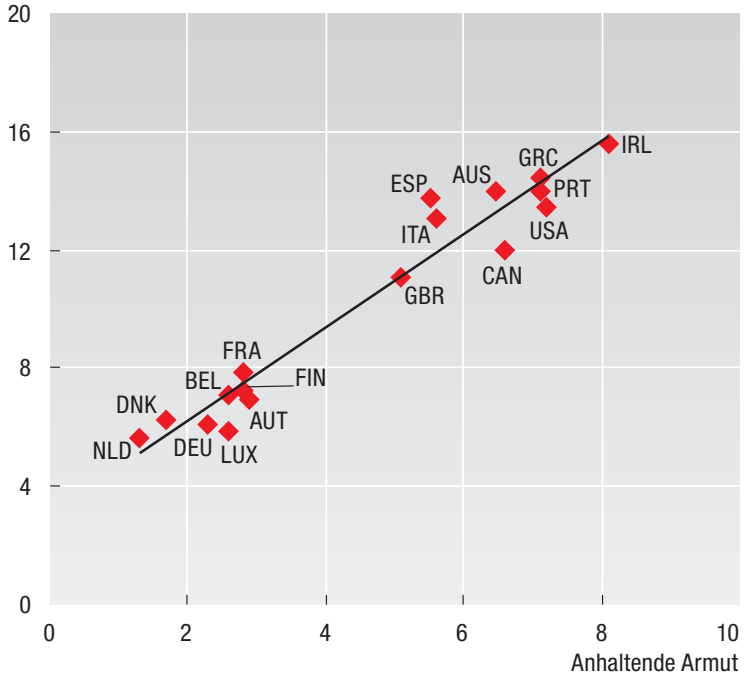

Quelle: Berechnungen auf der Grundlage des Haushaltspanels der Europäischen Gemeinschaften für die europäischen Länder und SLID für Kanada (CNEF, Fernzugriff). Die Schätzungen für die Vereinigten Staaten und Australien, entsprechend SIPP bzw. Hilda, stammen von John Iceland (University of Maryland) und Bruce Headey (University of Melbourne).

Weitere Informationen - Burkhauser, R. (2001), "What Policymakers Need to Know about Poverty Dynamics”, Journal of Policy Analysis and Management. OECD (2006), "Soziale Implikationen von Politiken zur Steigerung der Beschäftigung", Kapitel 5 in OECD-Beschäftigungsausblick, Paris. 


\section{Definition und Messung}

Die Wohnkosten sind entscheidende Bestimmungsfaktoren der Lebensbedingungen von Einzelpersonen und Haushalten. Der nachstehend verwendete Hauptindikator der Wohnkosten ist der Anteil am Haushaltseinkommen, der zur Finanzierung des Wohnens aufgewendet wird, und stützt sich auf Daten aus den jährlichen Volkswirtschaftlichen Gesamtrechnungen der OECD-Länder. Die Wohnkosten der privaten Haushalte, wie sie in den Volkswirtschaftlichen Gesamtrechnungen definiert sind, umfassen die tatsächlichen und die kalkulatorischen Mieten (d.h. das Mietäquivalent, das Wohneigentümer für eine Wohneinheit bezahlen müssten, die die gleichen Merkmale aufweist wie die, die sie besitzen), die Instandhaltungs- und Reparaturkosten sowie die Wasser-, Strom-, Gas- und sonstigen Brennstoffkosten. Nicht inbegriffen sind Zins- und Tilgungszahlungen für Immobilienkredite, da es im Fall ihrer Berücksichtigung zu einer Doppelzählung mit den kalkulatorischen Mieten kommen würde. Kalkulatorische Mieten stellen insofern eine bessere Messgröße der "realen" Wohnkosten dar, als ein gewisser Teil der Kredittilgungszahlungen in Wirklichkeit als Ersparnis der Haushalte zu sehen ist. Auf Grund starker Verzögerungen bei der Datenerfassung und -verbreitung reichen die Daten aus den Volkswirtschaftlichen Gesamtrechnungen für die meisten Länder nur bis zum Jahr 2003.

Informationen zu den Wohnkosten können auch aus Erhebungen über Einkommen und Ausgaben der privaten Haushalte entnommen werden. Erhebungsdaten haben den Vorteil, dass sie eine Analyse der Wohnkosten nach bestimmten Haushalts- bzw. individuellen Merkmalen ermöglichen. Der Nachteil dabei ist, dass in den verschiedenen Ländern unterschiedliche Kostenpunkte berücksichtigt werden (öffentliche Mietzuschüsse, Gebühren der Versorgungsunternehmen oder Tilgungszahlungen). Dieser Abschnitt liefert Informationen zur Höhe der Mietkosten im Verhältnis zum Einkommen an bestimmten Punkten der Verteilung der (äquivalisierten) verfügbaren Einkommen. Die Berechnungen stützen sich auf Mikrodaten aus der Luxemburger Einkommensstudie und nationalen Erhebungen (Frankreich und Kanada) sowie auf veröffentlichte Übersichtstafeln für die Vereinigten Staaten (die auf ähnlichen, aber nicht vollständig vergleichbaren Definitionen beruhen). Abgesehen von Definitionsunterschieden wird die Vergleichbarkeit dieser Erhebungsdaten auch durch unterschiedliche Stichprobengrößen und Stich probenfehler beeinträchtigt.

Infolge des in jüngster Zeit in den meisten OECD-Ländern verzeichneten steilen Anstiegs der Immobilienpreise und der Mieten hat die Frage der Erschwinglichkeit des Wohnens an Bedeutung gewonnen. Wohnraum ist sowohl der größte Ausgaben- als auch Vermögensfaktor der privaten Haushalte. Folglich können gestiegene Wohnkosten einerseits den finanziellen Spielraum der privaten Haushalte einengen, die nicht Eigentümer ihres Hauptwohnsitzes sind, und andererseits das Vermögen der Haushalte erhöhen, bei denen dies der Fall ist. Im Durchschnitt der 15 Länder, die in Abbildung EQ8.1 berücksichtigt sind, haben sich die Wohnkosten im Verhältnis zum verfügbaren Einkommen der privaten Haushalte seit 1995 etwas erhöht, von fast $20 \%$ im Jahr 1993 auf über $21 \%$ im Jahr 2003. Im Vereinigten Königreich setzte sich dieser Anstieg bis 2005 fort, in Frankreich und in Deutschland bis 2004. Der Großteil dieser Zunahme ist offenbar auf höhere kalkulatorische Mieten für Wohneigentümer zurückzuführen, während sich der Anstieg der tatsächlich von den Haushalten bezahlten Mieten zwischen 1995 und 2003 nur geringfügig beschleunigte (von durchschnittlich $4,1 \%$ auf $4,3 \%$ des verfügbaren Einkommens der Haushalte).

Die Inflation der Wohnimmobilien preise wirkt sich unterschiedlich auf die Lebensbedingungen der Haushalte aus, je nachdem ob sie Wohneigentümer oder Mieter sind und ob sie auf dem freien Markt mieten oder im öffentlichen Wohnungsbau. Die Wohneigentumsstrukturen variieren stark im OECD-Ländervergleich. Der Anteil der vom Eigentümer bewohnten Wohneinheiten liegt in Mexiko, der Slowakischen Republik, Griechenland, Frankreich, Finnland, Norwegen und den Vereinigten Staaten über $60 \%$, in Deutschland jedoch unter 40\% (Abb. EQ8.2). Sozialwohnungen spielen eine wichtige Rolle in Kanada ebenso wie in
Frankreich, Finnland, Dänemark und Polen. Die Bereitstellung von Sozialwohnungen, die unter Marktpreis vermietet werden, entspricht zwar einer Subvention für die privaten Haushalte, die allerdings häufig nicht den ärmsten Haushalten zugute kommt: In Frankreich entfallen beispielsweise $80 \%$ der Sozialwohnungen auf Haushalte, die nicht einkommensarm sind, während weniger als ein Drittel der einkommensarmen Haushalte in Sozialwohnungen lebt.

Die Mietkosten belasten einkommensschwache Haushalte stärker. Im Durchschnitt der 16 in Abbildung EQ8.3 dargestellten OECD-Länder entfielen auf die Mietkosten Anfang dieses Jahrzehnts mehr als 30\% des Einkommens der Haushalte im untersten Quintil der Verteilung, gegenüber $22 \%$ bzw. $16 \%$ im mittleren und im oberen Quintil. Wenn diese Kosten einen bestimmten Prozentsatz des Haushaltseinkommens übersteigen, wird Wohnraum unerschwinglich (ICCRSS, 2001). Einige OECD-Länder arbeiten mit spezifischen Maßen der Erschwinglichkeit von Wohnraum: In Kanada gaben 2001 rd. 35\% der Haushalte mindestens $30 \%$ ihres Einkommens vor Steuern für Wohnraum aus, wesentlich mehr als 10 Jahre zuvor, aber weniger als 1996; in Australien wurden 8,8\% der Haushalte im Jahr 2004 der Kategorie "Housing Stress" zugeordnet (Personen in den untersten $40 \%$ des Einkommensspektrums, die mehr als 30\% ihres Einkommens für Wohnraum ausgeben).

Statusindikatoren: Materielle Entbehrung (EQ1), Verdienstungleichheit (EQ2), Armutspersistenz (EQ7).

Reaktionsindikatoren: Öffentliche Sozialausgaben (EQ5). 
EQ8.1 Steigende Wohnkosten in den meisten OECD-Ländern

Wohnausgaben in Prozent des verfügbaren Einkommens der Haushalte, 1995-2005

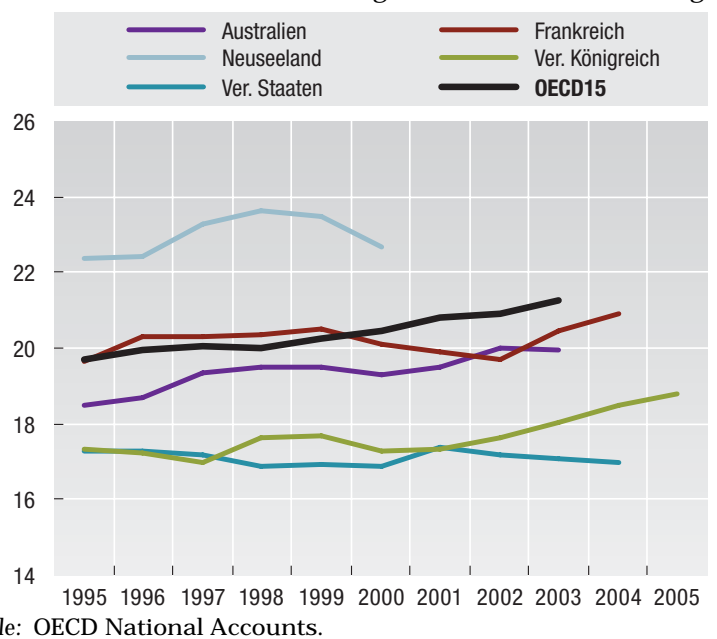

EQ8.2 Unterschiedliche Wohneigentumsstrukturen

Aufschlüsselung des Wohnungsbestands, in Prozent

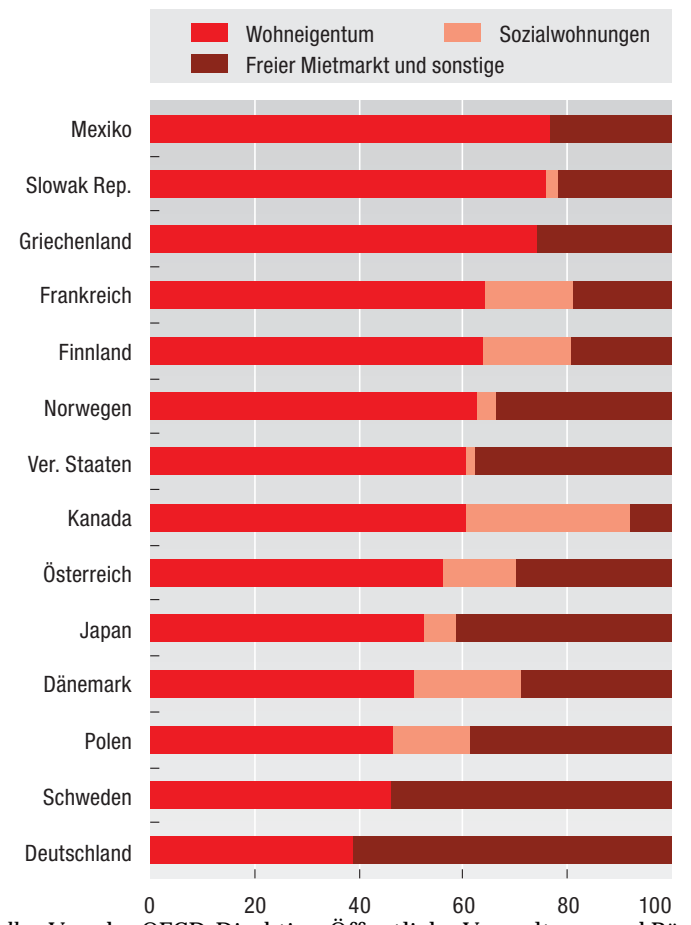

Quelle: Von der OECD-Direktion Öffentliche Verwaltung und Räumliche Entwicklung im Rahmen des Projekts über "sozialverträgliches Wohnen" gesammelte Daten.

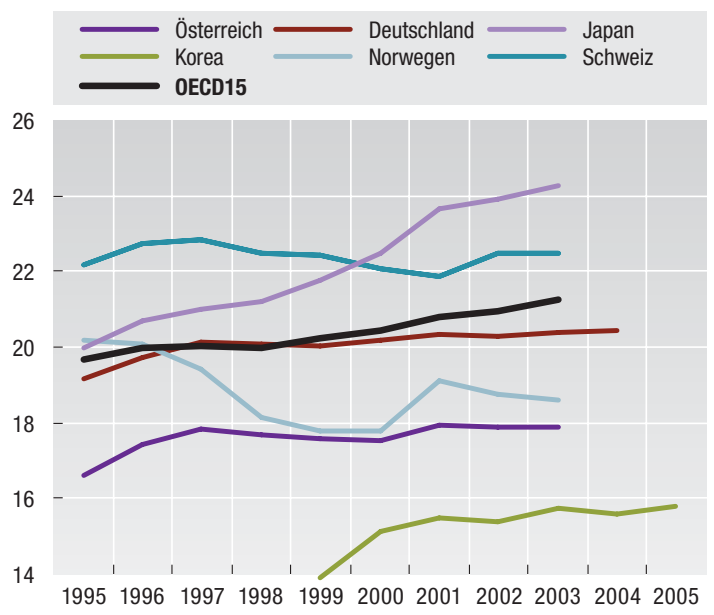

\section{EQ8.3 Einkommensschwache Mieter geben einen größeren Teil ihres Einkommens für die Miete aus als wohlhabende}

M ietkosten in Prozent des verfügbaren Nettoeinkommens der Mieter nach Einkommensquintil

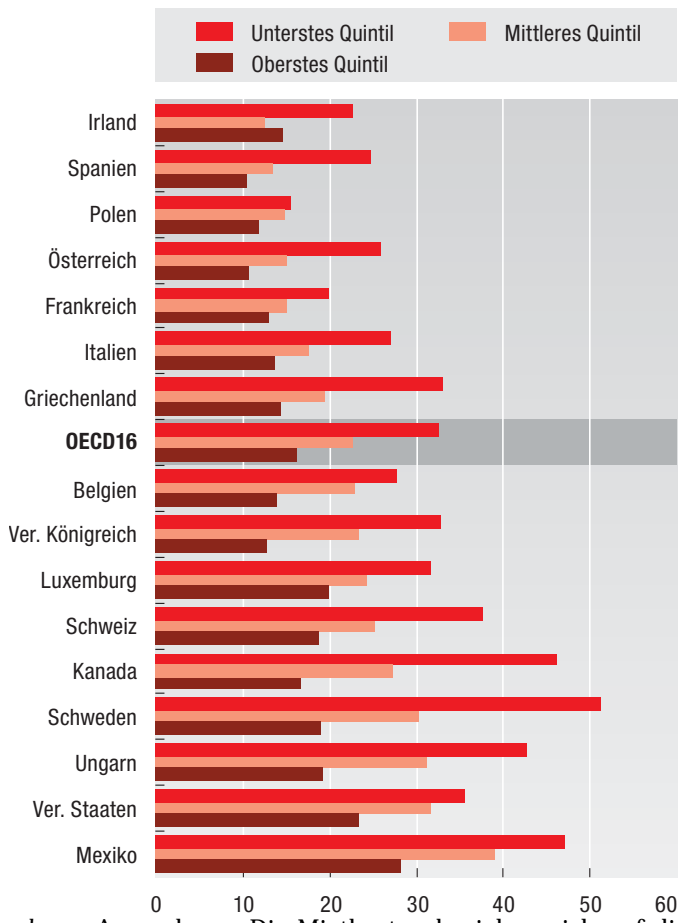

Anmerkung: Anmerkung: Die Mietkosten beziehen sich auf die gezahlten Nettomieten (in einigen Ländern einschließlich der Nebenkosten). Die Einstufung der Individuen erfolgte entsprechend ihres äquivalisierten verfügbaren Einkommens, außer in den Vereinigten Staaten (wo sie sich nach dem diskreten Niveau des Bruttohaushaltseinkommens richtet).

Quelle: Berechnungen auf der Basis der Luxemburger Einkommensstudie (LIS) und nationaler Daten.

$$
\text { StatLink } 2 \text { http://dx.doi.org/10.1787/562376451186 }
$$

Weitere Informationen $\square$ Harding, A., B. Phillips und S. Kelly (2004), Trends in Housing Stress, NATSEM, Canberra. $\square$ Interdisciplinary Centre for Comparative Research in the Social Sciences - ICCRSS (2001), Housing Stress: An Overview of Risk Populations and Policies in the EU, Wien. 


\section{Definition und Messung}

Mit der Ersatzquote der Altersrente kann gemessen werden, wie effektiv ein Rentensystem bei der Gewährleistung eines Einkommensersatzes für den Verdienst ist, der vor dem Renteneintritt die Haupteinkommensquelle darstellte. Beim hier verwendeten Indikator handelt es sich um die erwartete Rente einer alleinstehenden Arbeitskraft mit voller Erwerbsbiographie im privaten Sektor, die im Alter von 20 Jahren in den Arbeitsmarkt eintritt. Er umfasst alle obligatorischen Bestandteile der öffentlichen und privaten Altersvorsorge; die freiwillige Altersvorsorge, die in vielen Ländern eine wichtige Rolle spielt, ist jedoch nicht inbegriffen. In diesem Indikator, der den langfristigen Kurs des Rentensystems aufzeigen soll, sind alle bereits gesetzlich verankerten Änderungen der Regeln und Parameter berücksichtigt; schrittweise in Kraft tretende Änderungen sind somit zum Zeitpunkt des Renteneintritts voll erfasst. Die Parameter beziehen sich auf eine Arbeitskraft, die 2004 in den Arbeitsmarkt eingetreten ist. Für jedes Land wird ein Standardkatalog ökonomischer Annahmen zu Grunde gelegt.

Die Ersatzquote wird definiert als die Rentenansprüche geteilt durch den Verdienst vor Renteneintritt. Sie wird hier jeweils für 0,5, 0,75, 1,0, 1,5, 2,0 und 2,5 des Durchschnittsverdiensts dargestellt, gemäß dem neu definierten OECD-Konzept des "Durchschnittsarbeiters". Die Indikatoren für die erwartete Ersatzquote sind sowohl brutto (d.h. vor Steuern) als auch netto angegeben (d.h. nach Berücksichtigung der Steuern und Sozialversicherungsbeiträge, die während der Erwerbstätigkeit auf den Verdienst und nach dem Renteneintritt auf die bezogenen Renten zu zahlen sind).

In Tabelle EQ9.1 sind die Bruttoersatzquoten bei der Altersrente im Verhältnis zum Verdienst in allen 30 OECDLändern dargestellt. Dabei lassen sich verschiedene Formen von Rentensystemen unterscheiden. In Irland, Neuseeland und dem Vereinigten Königreich zahlt das Rentensystem allen Rentnern unabhängig von ihrer Verdienstbiographie einen Einheitsbetrag. Dies bedeutet, dass die Ersatzquote mit steigendem Verdienst sinkt. Diese Länder haben öffentliche Pauschalrentensysteme (die für jedes Beitrags- oder Wohnsitzjahr den gleichen Betrag auszahlen) oder bedürftigkeitsabhängige Systeme (in denen einkommensschwache Rentner höhere Leistungen beziehen). In Finnland, Italien und den Niederlanden sind die Ersatzquoten im gesamten Verdienstspektrum hingegen sehr ähnlich, d.h. die Höhe der Rente ist stark von der Höhe des früheren Verdiensts abhängig. Das polnische System setzt sich aus einer öffentlichen und einer privaten Komponente mit Beitragsprimat zusammen; die Bruttoersatzquoten verändern sich folglich nicht mit dem Verdienst. Die Systeme anderer Länder nehmen eine Zwischenposition ein: Frankreich und Deutschland gelten beide üblicherweise als Länder mit einer starken Sozialversicherungstradition; die Beitragsbemessungsgrenzen (die bei rd. 125\% bzw. 150\% des Durchschnittsverdiensts angesetzt sind), zu denen in Frankreich die großzügigen Mindestrenten hinzukommen, haben jedoch zur Folge, dass die Ersatzquoten anders als in der vorstehenden Gruppe von Ländern mit verdienstabhängigen Renten bei steigendem Verdienst sinken.

Im öffentlichen Rentensystem der Vereinigten Staaten kommt eine Rentenformel mit starker Umverteilungskomponente zur Anwendung. Bei der Hälfte des Durchschnitts- verdiensts beträgt die Ersatzquote $50 \%$, beim Durchschnittsverdienst sinkt sie auf $40 \%$ und beim Doppelten des Durchschnittsverdiensts auf $30 \%$. Japan hat ein zweistufiges öffentliches Rentensystem mit einer Pauschal- und einer verdienstabhängigen Komponente. Daraus ergibt sich eine ähnliche Ersatzquotenstruktur wie in den Vereinigten Staaten.

Für den Einzelnen kommt es auf die Nettoersatzquote an, weil diese über den Lebensstandard während der Rente im Vergleich zur Erwerbstätigkeit entscheidet (Abb. EQ9.2). Im Durchschnitt der OECD-Länder sind die Nettoersatzquoten bei einem durchschnittlichen Verdienst 15\% höher als die Bruttoersatzquoten; in Belgien, Deutschland und Frankreich sind sie deutlich höher. Bei Geringverdienern ist der Effekt der Steuern und Sozialversicherungsbeiträge weniger ausgeprägt, weil sie in der Regel weniger Steuern und Beiträge zahlen als Durchschnittsverdiener.

Bei einem durchschnittlichen Verdienst liegt die Nettoersatzquote im OECD-Durchschnitt bei $71 \%$. Dabei gibt es erhebliche Unterschiede: Die Grundrentensysteme in Irland und Neuseeland gewährleisten nur $40 \%$ oder weniger, wohingegen die Rentenansprüche in Griechenland und der Türkei höher sind als der frühere Verdienst.

Statusindikatoren: Generationenmobilität (EQ4), Lebenserwartung (HE1).

Reaktionsindikatoren: Öffentliche Sozialausgaben (EQ5), Gesamtsozialausgaben (EQ6). 
EQ9.1 Großzügigere Renten für Arbeitskräfte mit geringerem Verdienst

Bruttoersatzquoten der obligatorischen Altersvorsorge, in Prozent des individuellen Bruttoverdiensts vor Renteneintritt, Männer

\begin{tabular}{|c|c|c|c|c|c|c|c|c|c|c|c|c|c|}
\hline & \multicolumn{6}{|c|}{ Früherer Verdienst, Vielfache vom Durchschnitt } & & \multicolumn{6}{|c|}{ Früherer Verdienst, Vielfache vom Durchschnitt } \\
\hline & 0.5 & 0.75 & 1 & 1.5 & 2 & 2.5 & & 0.5 & 0.75 & 1 & 1.5 & 2 & 2.5 \\
\hline Australien & 70.7 & 52.3 & 43.1 & 33.8 & 29.2 & 26.5 & Niederlande & 80.6 & 81.5 & 81.9 & 82.4 & 82.6 & 82.7 \\
\hline Belgien & 57.3 & 40.9 & 40.4 & 31.3 & 23.5 & 18.8 & Norwegen & 60.4 & 54.3 & 51.9 & 42.4 & 34.6 & 27.7 \\
\hline Dänemark & 121.6 & 92.4 & 77.8 & 63.7 & 59.9 & 57.7 & Österreich & 80.1 & 80.1 & 80.1 & 78.5 & 58.8 & 47.1 \\
\hline Deutschland & 39.9 & 39.9 & 39.9 & 39.9 & 30.0 & 24.0 & Polen & 61.2 & 61.2 & 61.2 & 61.2 & 61.2 & 57.5 \\
\hline Finnland & 71.3 & 63.4 & 63.4 & 63.4 & 63.4 & 63.4 & Portugal & 70.4 & 68.3 & 67.8 & 66.9 & 66.0 & 65.4 \\
\hline Frankreich & 63.8 & 51.2 & 51.2 & 46.9 & 44.7 & 43.4 & Schweden & 79.3 & 66.8 & 62.5 & 65.4 & 66.8 & 67.6 \\
\hline Giechenland & 95.7 & 95.7 & 95.7 & 95.7 & 95.7 & 95.7 & Schweiz & 62.5 & 62.1 & 58.4 & 40.7 & 30.5 & 24.4 \\
\hline Irland & 65.0 & 43.3 & 32.5 & 21.7 & 16.2 & 13.0 & Slowak. Rep. & 56.7 & 56.7 & 56.7 & 56.7 & 56.7 & 56.7 \\
\hline Island & 109.9 & 85.8 & 77.5 & 74.4 & 72.9 & 71.9 & Spanien & 81.2 & 81.2 & 81.2 & 81.2 & 67.1 & 53.7 \\
\hline Italien & 67.9 & 67.9 & 67.9 & 67.9 & 67.9 & 67.9 & Tschech. Rep. & 78.8 & 59.0 & 49.1 & 36.4 & 28.9 & 24.4 \\
\hline Japan & 47.8 & 38.9 & 34.4 & 29.9 & 27.2 & 21.8 & Ungarn & 73.0 & 73.0 & 73.0 & 73.0 & 73.0 & 73.0 \\
\hline Kanada & 75.4 & 54.4 & 43.9 & 29.6 & 22.2 & 17.8 & Türkei & 72.5 & 72.5 & 72.5 & 72.5 & 72.5 & 71.7 \\
\hline Korea & 88.8 & 69.2 & 59.4 & 49.6 & 40.1 & 32.1 & Ver. Königreich & 53.4 & 37.8 & 30.8 & 22.6 & 17.0 & 13.6 \\
\hline Luxemburg & 99.8 & 92.1 & 88.3 & 84.5 & 82.5 & 76.2 & Ver. Staaten & 55.2 & 45.8 & 41.2 & 36.5 & 32.1 & 28.2 \\
\hline Mexiko & 52.8 & 37.3 & 35.8 & 34.4 & 33.6 & 33.2 & & & & & & & \\
\hline Neuseeland & 79.0 & 52.7 & 39.5 & 26.3 & 19.8 & 15.8 & OECD & 75.2 & 61.6 & 56.7 & 51.1 & 47.3 & 44.2 \\
\hline
\end{tabular}

EQ9.2 Für Durchschnittsverdiener beträgt die Nettoersatzquote in den OECD-Ländern 71\%

Nettoersatzquote nach Verdienstniveau, obligatorische A Itersvorsorge, in Prozent des individuellen Nettoverdiensts vor Renteneintritt, Männer
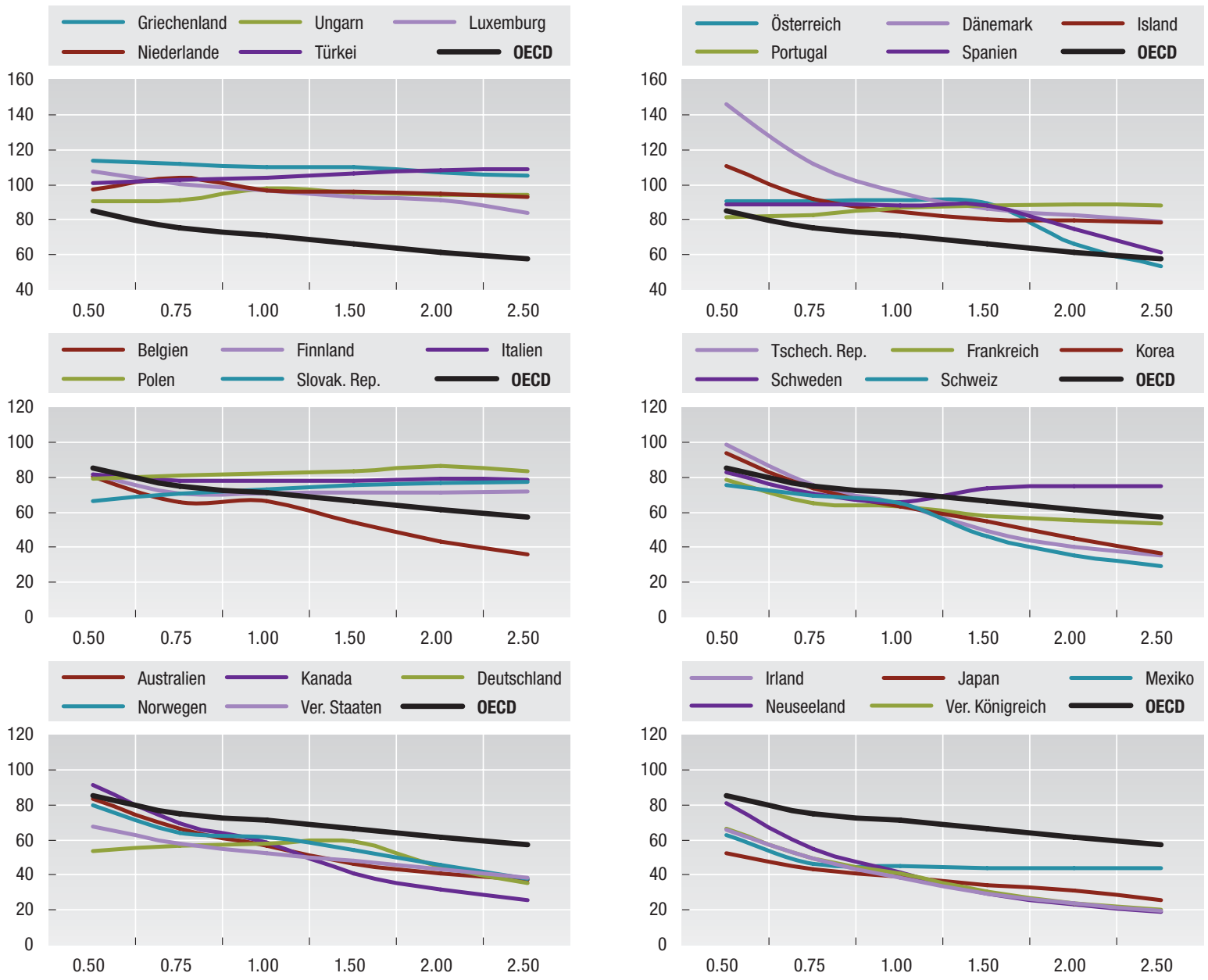

Quelle: OECD (2007, erscheint demnächst), Renten auf einen Blick: Staatliche Politik im OECD-Ländervergleich, Paris (www.oecd.org.els/social/ageing/PAG).

StatLink 2 http://dx.doi.org/10.1787/041567813082

Weitere Informationen — OECD (2000), Reforms for an Ageing Society, Paris. — OECD (2001), A geing and Income: Financial Resources and Retirement in 9 OECD Countries, Paris. Whitehouse, E. (2004), “The Value of Pension Entitlements: A Model of Nine OECD Countries", OECD Social, Employment and Migration Working Paper, Paris (www.oecd.org/els/workingpapers). 



\section{Definition und Messung}

Die Lebenserwartung ist der allgemeinste und bekannteste Indikator des Gesundheitszustands der Bevölkerung. Veränderungen der Lebenserwartung stehen mit einer ganzen Reihe interdependenter Variablen in Zusammenhang, wie z.B. Lebensstandard, Lebensweisen und Zugang zu qualitativ hochwertigen Gesundheitsleistungen. Da sich die fundamentalen sozioökonomischen Faktoren nicht von einem Tag auf den anderen wandeln, sind Veränderungen der Lebenserwartung am besten über lange Zeiträume hinweg zu beurteilen.

Die hier vorgestellten Indikatoren, die Lebenserwartung bei der Geburt und mit 65 Jahren, sind definiert als die durchschnittliche Zahl der einer Person voraussichtlich verbleibenden Lebensjahre gemäß der altersspezifischen Mortalitätsrate in einem gegebenen Land und in einem bestimmten Jahr. Sie berücksichtigen nicht den Effekt eines künftigen Rückgangs der altersspezifischen Sterberaten. Jedes Land berechnet die Lebenserwartung nach etwas anderen Methoden. Diese methodischen Abweichungen können die Vergleichbarkeit der übermittelten Schätzungen beeinträchtigen, da sie den Indikator der Lebenserwartung für ein Land um den Bruchteil eines Jahres verändern können.

Die Lebenserwartung bei der Geburt ist in den vergangenen 40 Jahren in den OECD-Ländern erheblich gestiegen, was sich durch einen starken Rückgang der Sterberaten in jedem Alter erklärt. Im Durchschnitt der OECD-Länder erhöhte sich die Lebenserwartung bei der Geburt seit 1960 für Frauen um 10,1 Jahre auf 81,1 Jahre im Jahr 2004 und für Männer um 9,4 Jahre auf 75,4 Jahre (Abb. HE1.1). 2004 lag die Lebenserwartung bei der Geburt für Frauen in Japan am höchsten (85,6 Jahre), gefolgt von Frankreich, Spanien, der Schweiz und Australien; für Männer war die Lebenserwartung in Island am höchsten (79,2 Jahre), gefolgt von Japan, der Schweiz, Schweden und Australien.

Der Anstieg der Lebenserwartung bei der Geburt im OECD-Raum ging mit einer bedeutenden Verringerung der Unterschiede zwischen den einzelnen Ländern einher, was durch das rasche Aufschließen zum Land mit den besten Ergebnissen bedingt war. In der Türkei erhöhte sich die Lebenserwartung bei der Geburt im Zeitraum 1960-2004 für Frauen und Männer zusammengenommen um 23 Jahre, während sie in Mexiko um mehr als 17 Jahre stieg. In beiden Ländern erklärte sich diese Erhöhung der Lebenserwartung in erster Linie durch die deutliche Reduzierung der Säuglingssterblichkeit.

Zwar folgt einem raschen Anstieg der Lebenserwartung während der Aufholphase normalerweise ein langsamerer Anstieg, doch gibt es kaum Anzeichen dafür, dass sich der Anstieg einem Plafond nähert: So ging die Zuwachsrate der Lebenserwartung bei der Geburt für japanische Frauen nach der Aufholphase auf die Hälfte zurück, setzt sich aber seither weiter bei rd. 3\% jährlich fort.

Da die Lebenserwartung bei der Geburt seit 1960 für Frauen etwas stärker stieg als für Männer, hat sich die Geschlechterdifferenz bei der Lebenserwartung im Zeitraum 1960-2004 im Durchschnitt geringfügig von
5,0 Jahren auf 5,7 Jahre vergrößert. Dieses Ergebnis verdeckt jedoch unterschiedliche Trends zwischen den ersten und den späteren Jahrzehnten. Während der Geschlechterunterschied bei der Lebenserwartung in vielen Ländern in den sechziger und siebziger Jahren erheblich zunahm (auf einen Höchststand von durchschnittlich 6,7 Jahren im Jahr 1980), hat er sich in den letzten 25 Jahren in mehreren OECD-Ländern verkleinert. Diese Verkleinerung ist z.T. auf die zunehmende Konvergenz im Risikoverhalten von Männern und Frauen (z.B. in Bezug auf das Rauchen) und die niedrigeren durch Herz-Kreislauf-Erkrankungen bedingten Mortalitätsraten unter Männern zurückzuführen.

Auch die Lebenserwartung älterer Personen hat sich dank eines besseren Zugangs zu Gesundheitsdiensten und des medizinischen Fortschritts, vor allem was die Behandlung von Herz-Kreislauf-Erkrankungen betrifft, in den letzten Jahrzehnten wesentlich erhöht. Im Jahr 2004 lag die Restlebenserwartung von Frauen im Alter von 65 Jahren durchschnittlich bei 19,5 Jahren, d.h. 4,5 Jahre mehr als 1960; Männer im selben Alter hatten eine Restlebenserwartung von 16 Jahren, d.h. 3,3 Jahre mehr als 1960 (Abb. HE1.2). Was die Lebenserwartung bei der Geburt betrifft, so hat sich der Geschlechterunterschied in Bezug auf die verzeichnete Zunahme der Lebenserwartung älterer Personen in mehreren OECD-Ländern seit Mitte der achtziger Jahre verkleinert, und dieser Trend wird sich den Projektionen zufolge künftig fortsetzen.

Statusindikatoren: Gesundheitliche Ungleichheit (HE6). Reaktionsindikatoren: Gesundheitsausgaben (HE2), Langzeitpflegebedürftige (HE5). 


\section{HE1.1 Frauen leben länger als Männer, und die Lebenserwartung bei der Geburt ist in den letzten Jahrzehnten für beide Geschlechter deutlich gestiegen}

Lebenserwartung bei der Geburt, in Jahren, Männer und Frauen, 1960 und $2004^{1}$

Frauen

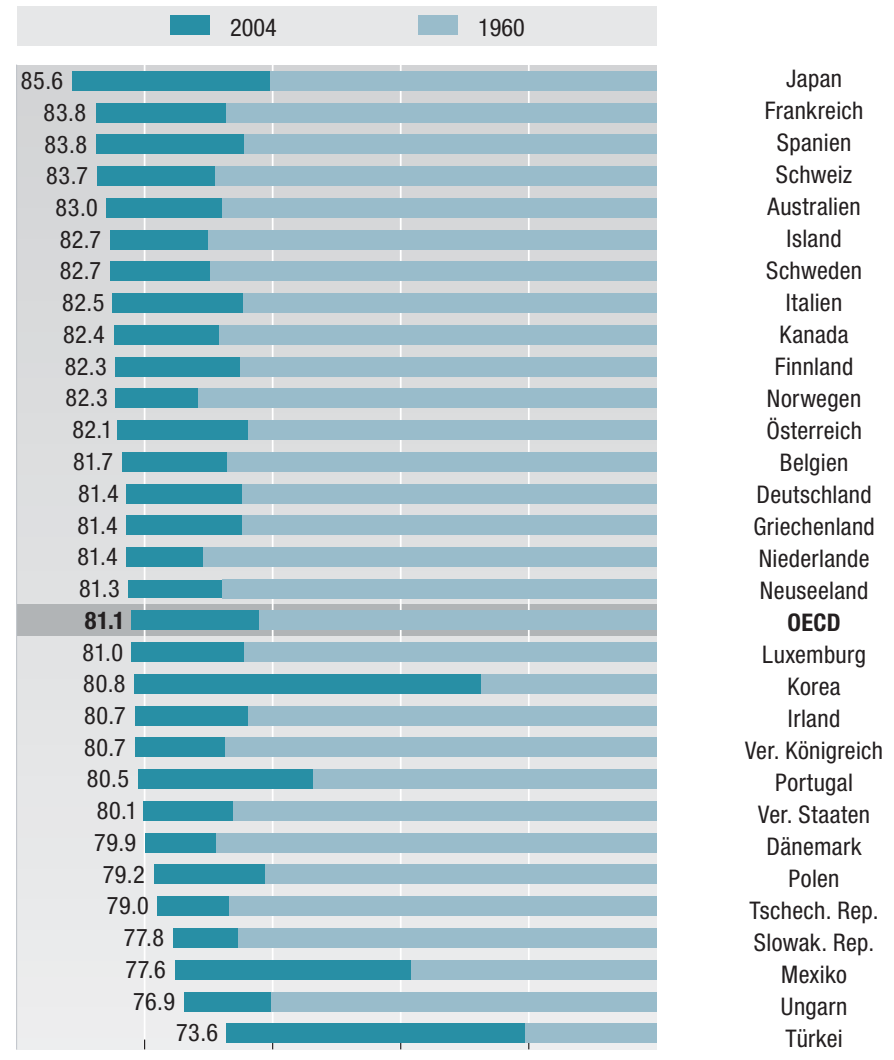

Männer

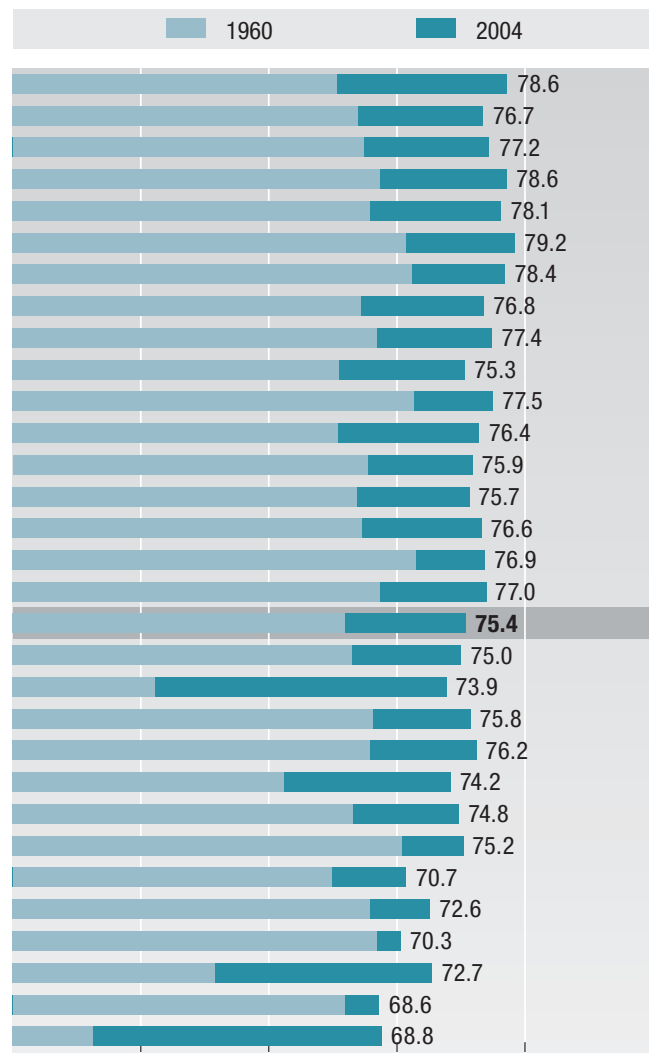

60

50

40

40

50

60

80

HE1.2 Eine längere Lebenserwartung auch für ältere Frauen

Lebenserwartung mit 65 Jahren, in Jahren, Männer und Frauen, $2004^{1}$

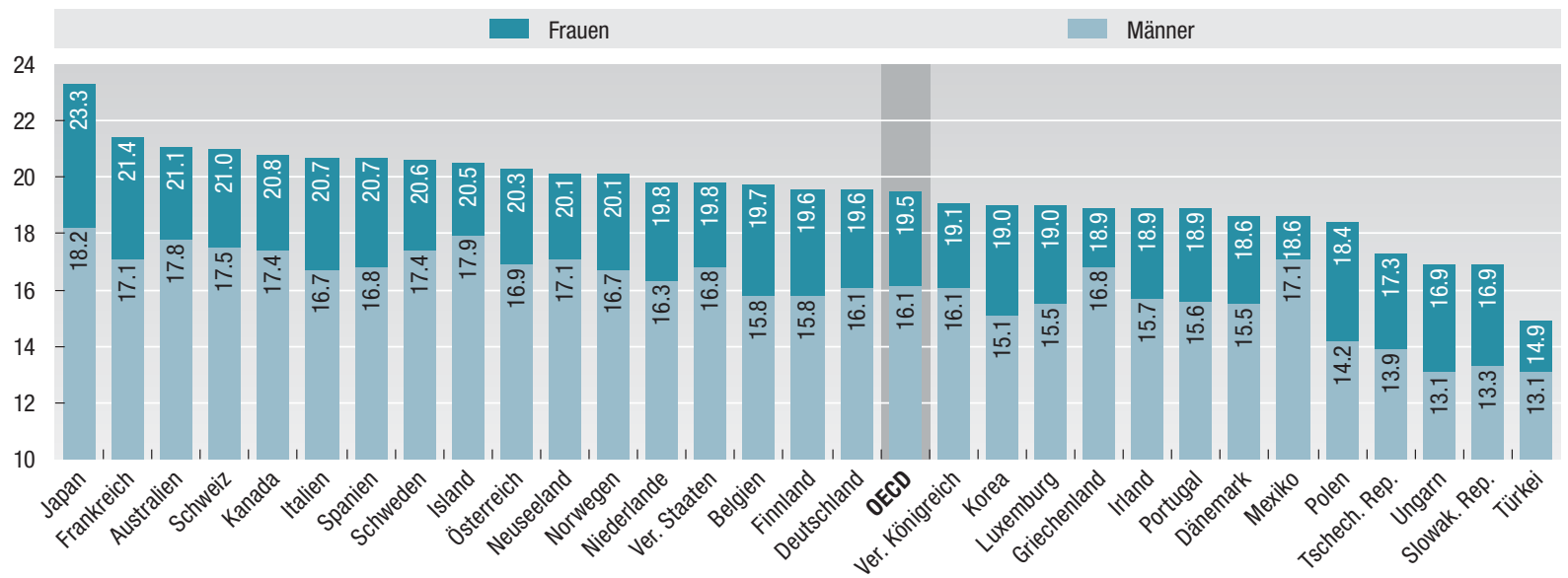

1. 2003 für Dänemark, Deutschland, Griechenland, Irland, Kanada, Korea, Luxemburg, Norwegen, Portugal, Schweiz, Slowakische Republik,

Tschechische Republik und Vereinigte Staaten; 2002 für Belgien, Finnland, Frankreich, Spanien und Vereinigtes Königreich; 2001 für Italien. Quelle: OECD (2006), OECD-Gesundheitsdaten 2006, CD-Rom, Paris (www.oecd.org/health/healthdata). StatLink 2

Weitere Informationen $\square$ OECD (2004), Towards High-Performing Health Systems, Paris. — OECD (2005), Gesundheit auf einen Blick - OECD-Indikatoren 2005, Paris. 


\section{Definition und Messung}

Mit den Gesamtausgaben für Gesundheit werden der Endverbrauch an Waren und Dienstleistungen der Gesundheitsversorgung (d.h. die laufenden Gesundheitsausgaben) sowie die Kapitalinvestitionen in die Gesundheitsinfrastruktur gemessen. Erfasst werden damit sowohl die öffentlichen als auch die privaten Ausgaben (unter Einschluss des Haushaltssektors) für medizinische Dienstleistungen und Waren, und Ausgaben für öffentliche Gesundheits- und Vorsorgeprogramme sowie Verwaltungsleistungen. Unberücksichtigt bleiben Ausgaben im Gesundheitsbereich, die im Zusammenhang mit Ausbildung, Forschung und umweltbezogenem Gesundheitsschutz stehen. Die beiden Hauptkomponenten der laufenden Gesamtgesundheitsausgaben sind: Ausgaben für die individuelle Gesundheitsversorgung und Ausgaben für Kollektivleistungen.

Zum Vergleich des Gesamtverbrauchsniveaus von Gesundheitswaren und -dienstleistungen in den einzelnen Ländern zu einem gegebenen Zeitpunkt werden die Pro-Kopf-Gesundheitsausgaben in eine gemeinsame Währung (US-\$) konvertiert und angepasst, um der unterschiedlichen Kaufkraft der nationalen Währungen in den einzelnen Ländern Rechnung zu tragen. Die gesamtwirtschaftlichen (BIP) Kaufkraftparitäten (KKP) werden als die am meisten verfügbaren und verlässlichsten Umrechnungsraten verwendet.

Im Jahr 2004 wendeten die OECD-Länder im Durchschnitt $8,9 \%$ ihres BIP für Gesundheitsausgaben auf. Dieser Anteil variiert zwischen den Ländern erheblich und reicht von 15,3\% in den Vereinigten Staaten bis zu unter $6 \%$ in der Slowakischen Republik und Korea (Abb. HE2.1). Hinter den Vereinigten Staaten rangierten in Bezug auf den höchsten prozentualen BIP-Anteil der Gesundheitsausgaben die Schweiz und Deutschland mit 11,6\% bzw. 10,9\% an nächster Stelle. 2004 wendeten acht Länder mehr als $10 \%$ des BIP für die Gesundheitsversorgung auf, während es 1997 lediglich drei Länder waren. Die öffentlichen Gesundheitsausgaben machten in Deutschland, Frankreich, Island und Norwegen mehr als $8 \%$ des BIP aus, in Korea und Mexiko hingegen nur etwa 3\%.

Der öffentliche Sektor finanziert in allen OECD-Ländern mit Ausnahme der Vereinigten Staaten und Mexikos weiterhin den Großteil der Gesundheitskosten. Im Durchschnitt wurden 2004 in den OECD-Ländern 73\% der Gesundheitsausgaben mit öffentlichen Mitteln finanziert. Dieser durchschnittliche öffentliche Anteil blieb im Zeitverlauf relativ konstant, wobei aber in mehreren Ländern erhebliche Veränderungen zu beobachten waren. Von wenigen Ausnahmen abgesehen ist zudem seit Anfang der neunziger Jahre der Anteil der öffentlichen Gesundheitsausgaben an den Gesamtausgaben der OECD-Länder konvergiert. Viele Länder, deren Anteil der öffentlichen Gesundheitsausgaben 1990 relativ hoch war, wie z.B. Polen, Ungarn und die Tschechische Republik, haben einen Rückgang zu verzeichnen. Andererseits wiesen mehrere Länder, in denen der Anteil der öffentlichen Gesundheitsausgaben 1990 gering war, im Zeitverlauf eine Zunahme dieses Anteils auf (z.B. Korea, die Vereinigten Staaten, Mexiko und die Schweiz). In Korea erhöhte sich der Anteil der öffentlichen Gesundheitsausgaben im Zeitraum 1990-2004 von 38\% auf etwas mehr als die Hälfte der gesamten Gesundheitsausgaben.

Im Zeitverlauf eintretende Veränderungen im Verhältnis Gesundheitsausgaben/BIP spiegeln den kombinierten Effekt der Trendentwicklungen von BIP und Gesundheitsausgaben wider. Zwischen 1990 und 2004 hat sich der für Gesundheitsausgaben aufgewendete Anteil am Nationaleinkommen in fast allen OECD-Ländern vergrößert, da die Zuwachsrate der Gesundheitsausgaben die Wachstumsrate der Gesamtwirtschaft überstieg. Im Durchschnitt der OECDLänder erhöhte sich der Ausgabenquotient von 7,0\% auf $8,9 \%$. Vor allem Norwegen, Island und die Vereinigten Staaten verzeichneten einen starken Anstieg, denn hier wuchsen die Gesundheitsausgaben doppelt so rasch wie das BIP. In Finnland und Italien erhöhte sich der BIP-Anteil der Gesundheitsausgaben dagegen nur geringfügig.

Abbildung HE2.2 zeigt einen positiven Zusammenhang zwischen Pro-Kopf-Netto-Nationaleinkommen (NNE) und Pro-Kopf-Gesundheitsausgaben in den OECD-Ländern. Auch wenn Länder mit höherem Pro-Kopf-NNE einen größeren Anteil ihres NNE für Gesundheitszwecke ausgeben, gibt es doch große Unterschiede zwischen den Ländern, da das NNE nicht der einzige Einflussfaktor ist. Der erwähnte Zusammenhang ist zudem in Ländern mit niedrigerem Einkommen stärker als in Ländern mit höherem Pro-Kopf-NNE. Unter Ländern mit einem Pro-Kopf-NNE von 25000 US-\$ KKP und darüber sind hinsichtlich der Gesundheitsausgaben bei einem bestimmten NNE-Niveau erhebliche Differenzen auszumachen. Zum Beispiel gibt Deutschland rund ein Drittel mehr für Gesundheitszwecke aus als Italien und Japan, trotz eines vergleichbaren Pro-Kopf-NNE.

Abbildung HE2.3 zeigt den Zusammenhang zwischen der Lebenserwartung bei der Geburt und den Pro-KopfGesundheitsausgaben in den einzelnen OECD-Ländern. Mit höheren Pro-Kopf-Gesundheitsausgaben wird generell eine höhere Lebenserwartung bei der Geburt assoziiert, obwohl dieser Zusammenhang in Ländern mit höheren Pro-KopfGesundheitsausgaben generell weniger deutlich ist. Hier wiederum ragen Japan und Spanien auf Grund ihrer im Verhältnis zum Niveau der Gesundheitsausgaben hohen Lebenserwartung heraus, während für die Vereinigten Staaten, Ungarn und die Türkei das Gegenteil zutrifft. Diese einfachen Korrelationen lassen sich natürlich nur vermuten, denn Unterschiede beim Pro-Kopf-NNE können sowohl die Lebenserwartung als auch die Pro-Kopf-Gesundheitsausgaben beeinflussen; neben dem Nationaleinkommen und den Gesundheitsausgaben müssen noch viele andere Faktoren herangezogen werden, um die zwischen den Ländern bei der Lebenserwartung bestehenden Unterschiede zu erklären.

Statusindikatoren: Lebenserwartung (HE1).

Reaktionsindikatoren: Langzeitpflegeausgaben (HE5), Öffentliche Sozialausgaben (EQ5). 
HE2.1 Höhere öffentliche und private Ausgaben für Gesundheit seit 1990

Öffentliche und private Ausgaben für Gesundheit, in Prozent des BIP, $2004^{1}$ (blaue Balken) und $1990^{2}$ (Rauten) Öffentlich

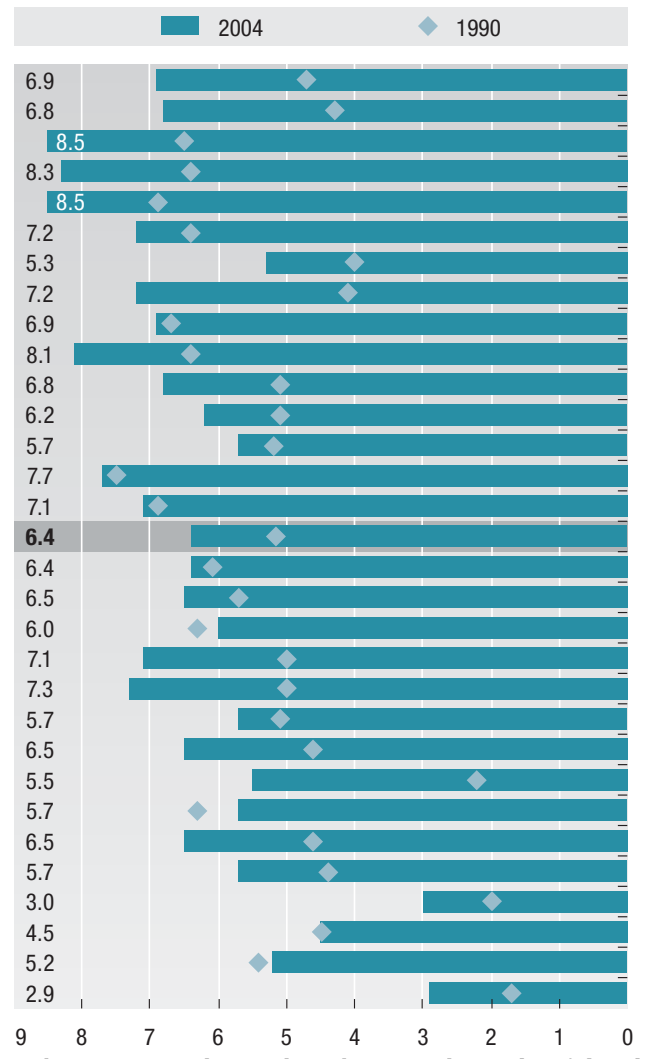

Ver. Staaten (15.4)
Schweiz (11.6)
Deutschland (10.9)
Frankreich (10.6)
Island (10.2)
Belgien (10.1)
Griechenland (10.0)
Portugal (10.0)
Kanada (9.9)
Norwegen (9.7)
Österreich (9.6)
Australien (9.2)
Niederlande (9.2)
Schweden (9.1)
Dänemark (8.9)
0ECD (8.9)
Italien (8.4)
Neuseeland (8.4)
Ungarn (8.3)
Ver. Königreich (8.3)
Luxemburg (8.1)
Spanien (8.1)
Japan (8)
Türkei (7.6)
Finnland (7.5)
Tschech. Rep. (7.3)
Irland (7.2)
Mexiko (6.5)
Polen (6.5)
Slowak. Rep. (5.9)
Korea (5.6)
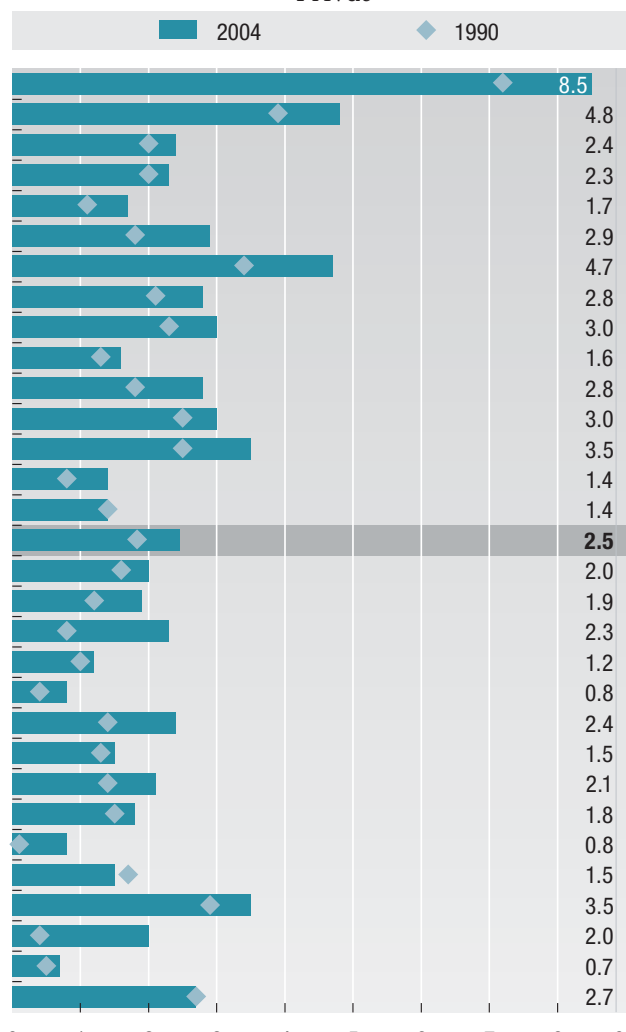

Anmerkung: Die Länder sind in absteigender Reihenfolge ihrer Gesamtgesundheitsausgaben von 2004 angegeben (Werte in Klammern in der mittleren Spalte).

1. 2003 für Australien, Belgien, Deutschland, Japan und die Slowakische Republik; laufende Ausgaben für Dänemark.

2. 1991 für Ungarn; 1995 für Belgien; 1997 für die Slowakische Republik.

\section{HE2.2 Pro-Kopf-Gesundheitsausgaben und -Nationaleinkommen, 2004}

Pro Kopf für NNE und Gesamtgesundheitsausgaben, $2004^{1}$, US-\$/KKP Gesamtgesundheitsausgaben pro Kopf (US-\$/KKP)

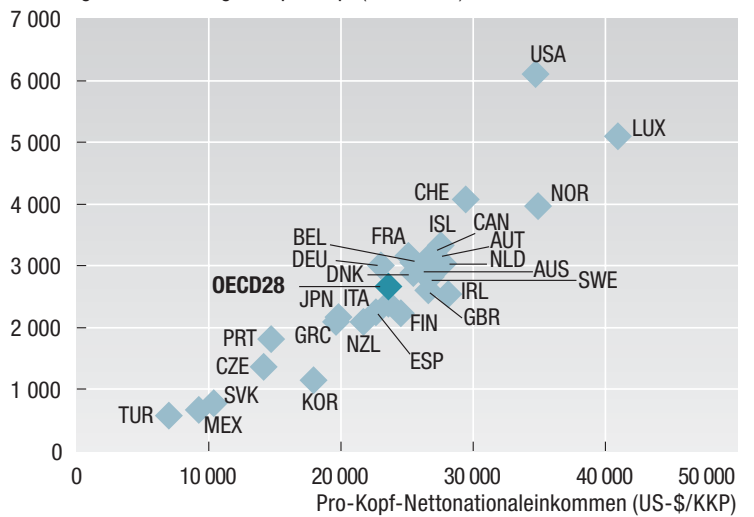

1. 2003 für Australien, Belgien, Deutschland, Japan und die Slowakische Republik.

Quelle: OECD (2006), OECD-Gesundheitsdaten 2006, Paris (www.oecd.org/health/healthdata),

S tat Link 2
HE2.3 Unterschiede zwischen den OECD-Ländern bei Gesundheitsausgaben und Gesundheitsergebnissen

Pro-Kopf-Gesundheitsausgaben und Lebenserwartung bei der Geburt, 2004 Gesamtgesundheitsausgaben pro Kopf (US-\$/KKP)

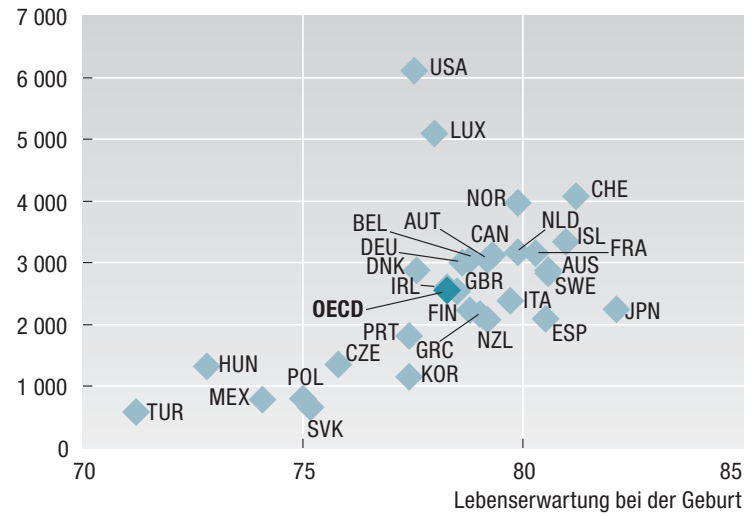

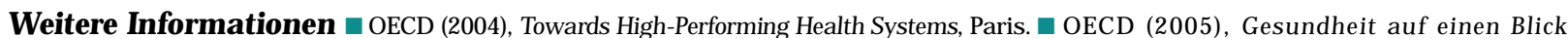
- OECD-Indikatoren 2005, Paris. 


\section{Definition und Messung}

Ein niedriges Geburtsgewicht ist der Definition der Weltgesundheitsorganisation (WHO) zufolge das Gewicht eines Säuglings bei der Geburt, das unabhängig von der Dauer der Schwangerschaft unter 2500 Gramm liegt. Dieser Grenzwert basiert auf epidemiologischen Untersuchungen des erhöhten Sterberisikos bei Säuglingen und dient dem internationalen Vergleich der Gesundheitsstatistiken. Die Zahl der Neugeborenen mit niedrigem Gewicht wird dann in Prozent aller Lebendgeburten ausgedrückt. Die Mehrzahl der Daten ist Geburtsregistern entnommen; im Fall der Niederlande ist die Datenquelle jedoch eine nationale Gesundheitserhebung.

Dieser Abschnitt enthält zudem Daten über die Säuglingssterblichkeit, d.h. die Zahl der Todesfälle bei Kindern unter einem Lebensjahr, die in einem gegebenen Jahr je 1000 Lebendgeburten auftreten. Ein Teil der internationalen Unterschiede bei den Säuglingssterberaten ist u.U. darauf zurückzuführen, dass zwischen den Ländern Differenzen in Bezug darauf bestehen, ob Frühgeburten statistisch als Lebendgeburten erfasst werden oder nicht; die Tatsache, dass in einigen Ländern, wie beispielsweise den Vereinigten Staaten und den nordischen Ländern, sehr kleine Frühgeborene mit relativ geringen Überlebenschancen als Lebendgeburten registriert werden, erhöht die Sterberaten im Vergleich zu anderen Ländern.

Ein niedriges Geburtsgewicht ist ein wichtiger Indikator für die Gesundheit eines Säuglings, da zwischen Geburtsgewicht und Säuglingsmortalität bzw. -morbidität ein Zusammenhang besteht. Es gibt zwei Kategorien von untergewichtigen Säuglingen, solche, die zu früh geboren werden, und solche, die auf Grund von verzögertem fetalen Wachstum oder IUWR (Intrauteriner Wachstumsretardierung) ein niedriges Gewicht aufweisen. Bei Säuglingen mit niedrigem Geburtsgewicht ist die Gefahr von Gesundheitsschäden bzw. das Sterberisiko größer, sie müssen nach der Geburt für längere Zeit im Krankenhaus bleiben und entwickeln mit höherer Wahrscheinlichkeit schwere Behinderungen (UNICEF und WHO, 2004). Zu den möglichen Bestimmungsfaktoren für ein niedriges Geburtsgewicht zählen der sozioökonomische Status der Eltern, demographische Faktoren (Alter der Mutter, Mehrfachgeburten usw.), individuelle Verhaltensweisen wie Rauchen und Alkoholkonsum sowie das Niveau der pränatalen Versorgung.

Im Jahr 2004 (bzw. dem letzten verfügbaren Jahr) gaben Island, Finnland, Korea und Schweden mit Werten von bis zu 4,5\% unter den OECD-Ländern den geringsten Anteil an Neugeborenen mit niedrigem Geburtsgewicht an. Japan, Ungarn und Griechenland sind am anderen Ende der Skala angesiedelt, wo die Rate der Neugeborenen mit niedrigem Geburtsgewicht $8 \%$ überschreitet (Abb. HE3.1). Die Türkei, die Vereinigten Staaten und das Vereinigte Königreich folgen dicht dahinter, in diesen Ländern wird bei nahezu 8\% aller Lebendgeburten ein niedriges Geburtsgewicht registriert. Der OECD-Durchschnitt liegt vergleichsweise bei $6,5 \%$.

Seit 1980 hat sich der Anteil der Neugeborenen mit niedrigem Geburtsgewicht in mehreren OECD-Ländern erhöht (Abb. HE3.2 und Abb. HE3.3). Hierfür gibt es mehrere Gründe. Erstens ist die Zahl der Mehrlingsgeburten, mit denen das Risiko von Frühgeburten und niedrigem Geburtsgewicht zunimmt, z.T. infolge der Zunahme von Fruchtbarkeitsbehandlungen stetig gestiegen. Zweitens tendieren Frauen mehr und mehr dazu, mit einer Schwangerschaft bis zu einem Alter von über 30 Jahren zu warten, was wiederum das Risiko von Geburten mit niedrigem Gewicht erhöht. Ein dritter Faktor ist, dass die neue medizinische
Technologie und die bessere pränatale Versorgung sehr kleinen Föten eine größere Chance bieten, lebend geboren zu werden.

Abbildung HE3.4 zeigt eine positive Korrelation zwischen dem Prozentsatz an Neugeborenen mit niedrigem Geburtsgewicht und den Sterberaten von Säuglingen. In der Regel verzeichnen Länder mit einem geringen Anteil an solchen Geburten auch verhältnismäßig niedrige Säuglingssterberaten. Das trifft beispielsweise auf die nordischen Länder zu. Japan stellt indessen eine Ausnahme dar, mit dem höchsten Anteil an mit niedrigem Gewicht geborenen Kindern, aber einer der niedrigsten Säuglingssterberaten. Japan, das historisch zur Ländergruppe mit einem geringen Anteil an Niedriggewichtgeburten zählte, erlebte mit einem Anstieg der Zahl der Säuglinge mit niedrigem Geburtsgewicht von rd. $5 \%$ der Neugeborenen in den späten siebziger Jahren auf über $9 \%$ im Jahr 2003 die stärkste Zunahme. Eine Reihe von Faktoren wird als Grund hierfür angeführt. Einer davon ist der wachsende Anteil von Raucherinnen unter jüngeren japanischen Frauen seit den siebziger Jahren; ein weiterer ist der deutliche Trend bei japanischen Frauen zu einer späteren Mutterschaft (Jeong und Hurst, 2001). Andererseits wurde auch geltend gemacht, dass die medizinische Versorgung für Neugeborene in Japan bei der Verringerung der Säuglingssterblichkeit besonders erfolgreich war, und dies trotz der Zunahme von Neugeborenen mit niedrigem Gewicht.

Vergleiche unterschiedlicher Bevölkerungsgruppen innerhalb der Länder legen den Schluss nahe, dass der Anteil der Neugeborenen mit niedrigem Gewicht auch durch Unterschiede in Bezug auf Bildung, Einkommen und ethnische Zugehörigkeit beeinflusst wird. In den Vereinigten Staaten ist die Rate bei schwarzen Säuglingen fast doppelt so hoch wie bei weißen; ähnliche Unterschiede sind auch zwischen der indigenen und der übrigen Bevölkerung in Australien und Mexiko beobachtet worden.

Statusindikatoren: Lebenserwartung (HE1). Responseindikatoren: Gesundheitsausgaben (HE2). 
HE3.1 Bis zu 1 von 10 Neugeborenen wiegt weniger als 2,5 kg

Zahl der Säuglinge mit niedrigem Geburtsgewicht je 1000 Lebendgeburten, 2004

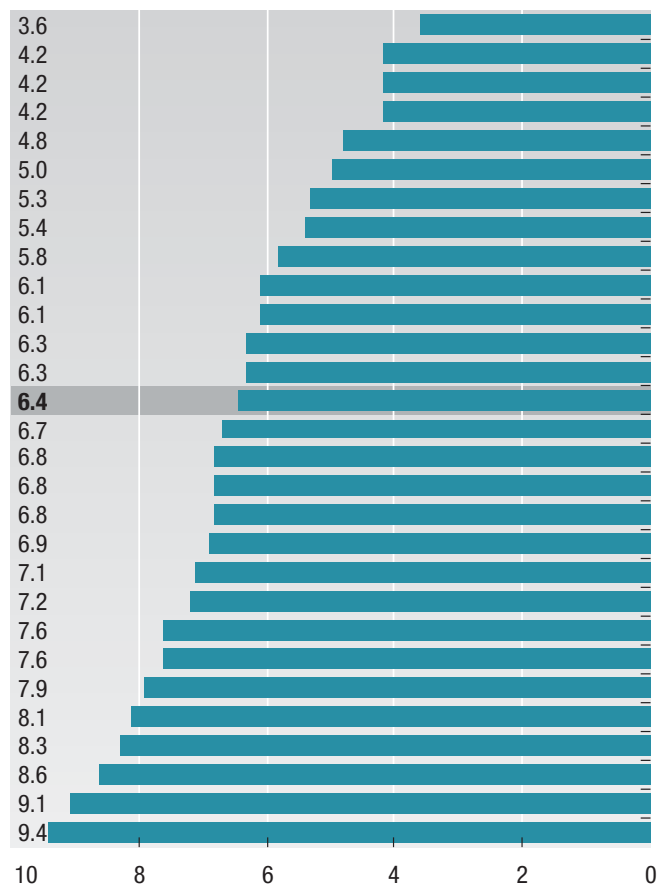

Prozentsatz der Neugeborenen mit einem Gewicht unter $2500 \mathrm{~g}$

1. 2003.

2. 2002 .

\section{HE3.3 Trends in Bezug auf Säuglinge mit niedrigem Geburtsgewicht, 1980-2004}

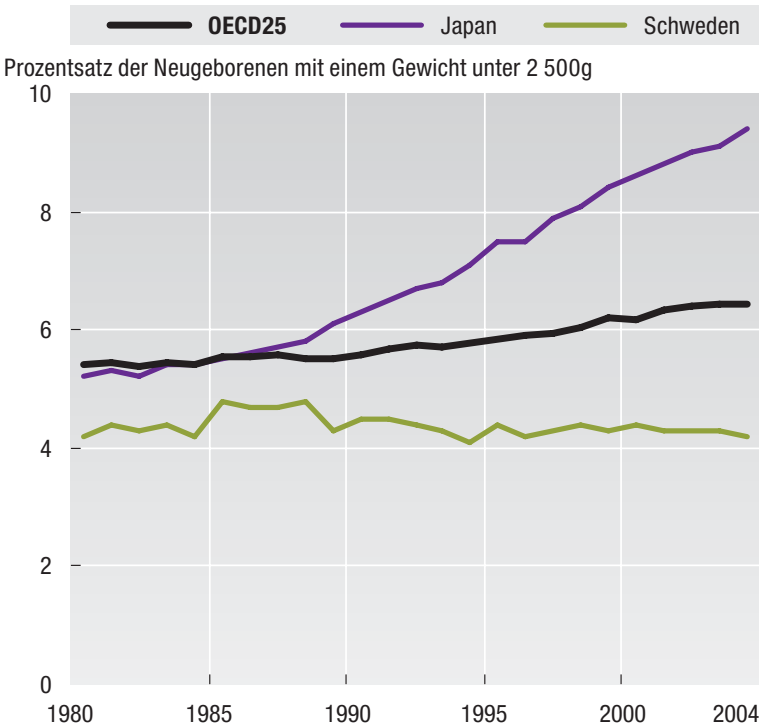

\section{HE3.2 Anstieg der Zahl der Säuglinge mit niedrigem Geburtsgewicht, 1980-2004}

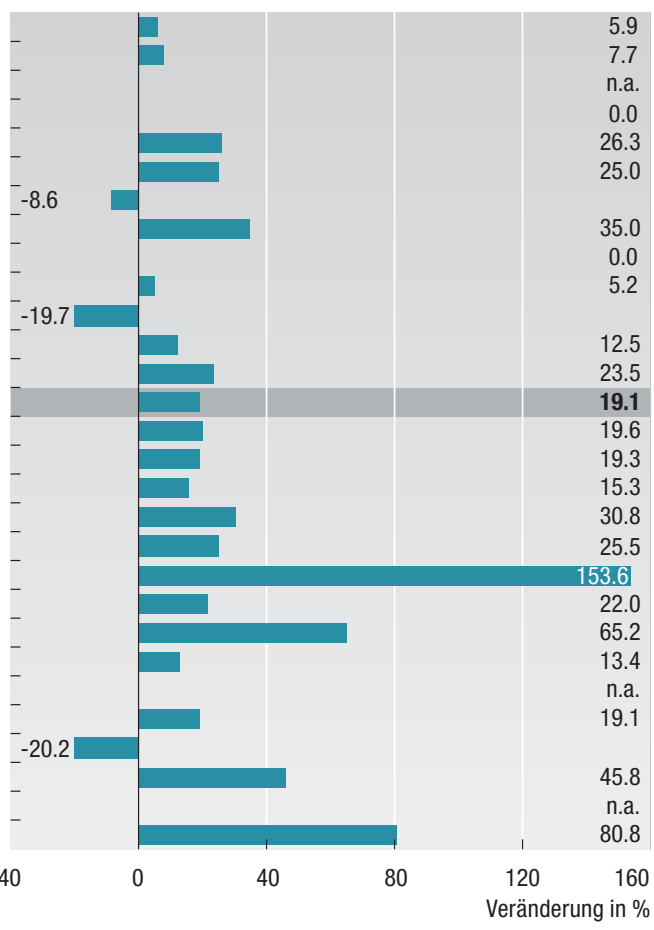

HE3.4 Niedriges Geburtsgewicht und Säuglingssterblichkeit, 2004

Säuglingssterblichkeit (Todesfälle je 1000 Lebendgeburten)

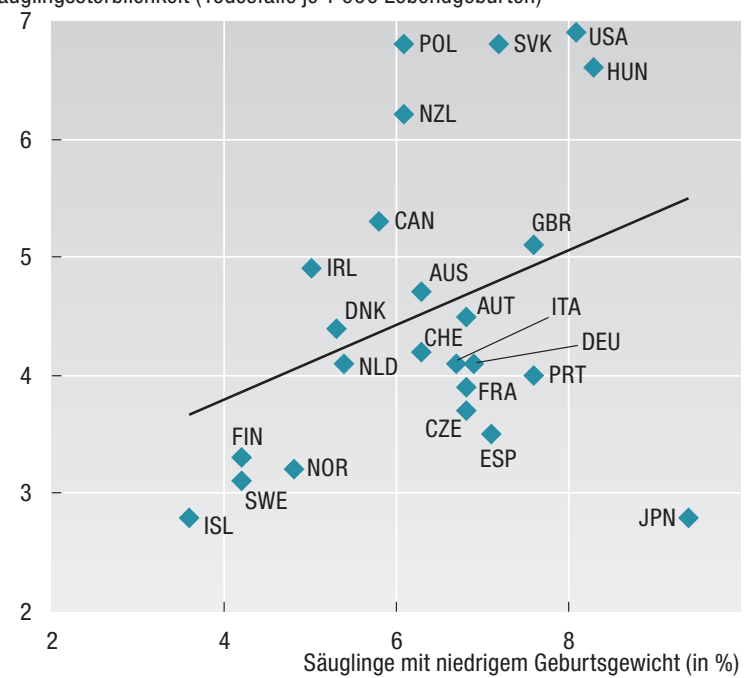

Quelle: OECD (2006), OECD-Gesundheitsdaten 2006, Paris (www.oecd.org/health/healthdata).

S tatLink 2 http://dx.doi.org/10.1787/128205010012

Weitere Informationen a Jeong, H.S. und J. Hurst (2001), "An Assessment of the Performance of the Japanese Health Care System", OECD Labour Market and Social Policy Occasional Paper, No. 56, Paris. n OECD (2004), Towards High-Performing Health Systems, Paris. OECD (2005), Gesundheit auf einen Blick - OECD-Indikatoren 2005, Paris. — UNICEF und WHO (2004), Low Birthweight: Country, regional and global estimates, UNICEF, New York. 


\section{Definition und Messung}

Messgrößen der krankheitsbedingten Fehlzeiten sind in mehrerer Hinsicht wichtig. Sie informieren über den Verlust an Arbeitskraft (d.h. entgangener Produktion) und den durch krankheitsbedingte Abwesenheit vom Arbeitsplatz entstehenden Kostendruck, und sie geben Aufschluss über die Gesundheit der Arbeitnehmer, den Grad ihrer Arbeitszufriedenheit und die Integration am Arbeitsplatz. Es gibt weder eine international vereinbarte Definition der krankheitsbedingten Fehlzeiten noch eine für internationale Vergleiche heranziehbare einheitliche Datenquelle. Die von den Krankenversicherungen oder den Unternehmen erfassten Fehlzeiten sind zwar für jedes Land die Hauptinformationsquelle, doch werden diese Daten durch die unterschiedlichen nationalen Praktiken bei der Erfassung derartiger Fehlzeiten beeinträchtigt. Eine bessere Alternative sind die im Rahmen von Umfragen bei privaten Haushalten von den Betroffenen selbst angegebenen Fehlzeiten, selbst wenn diese durch geringe Stich probengrößen, Unterschiede im Hinblick auf die Frequenz der Durchführung der Umfragen und den Wortlaut der Fragestellungen beeinträchtigt werden können.

In diesem Abschnitt werden zwei auf Arbeitsmarkterhebungen (EU-Arbeitskräfteerhebung für 22 europäische Länder und nationale Erhebungen für die übrigen Länder) basierende Indikatoren für die krankheitsbedingten Fehlzeiten vorgestellt. Der erste Indikator für Vollzeitbeschäftigte bezieht sich auf Arbeitskräfte, die angaben, in der Referenzwoche der Erhebung ihrem Arbeitsplatz krankheitsbedingt vorübergehend ferngeblieben zu sein. Die Daten aus den europäischen Ländern erfassen krankheitsbedingte Fehlzeiten mit einer Dauer von einer ganzen Woche bzw. eines Teils davon, während sich die Daten einiger Nicht-EU-Länder oft auf die erste Kategorie beschränken. Der gezeigte Indikator ist die durchschnittliche Zahl des jährlichen Arbeitstageausfalls je Arbeitnehmer. Der zweite Indikator für als nichterwerbstätig eingestufte Personen bezieht sich auf diejenigen, die "Krankheit und Erwerbsunfähigkeit" als Hauptgrund ihrer Nichterwerbstätigkeit angeben; der gezeigte Indikator ist der Bevölkerungsanteil der Personen, die sich in dieser Situation befinden. Bei Arbeitskräfteerhebungen kann das erfasste Ausmaß krankheitsbedingter Fehlzeiten insofern unvollständig sein, als dabei lediglich der "Hauptgrund" der Abwesenheit vom Arbeitsplatz angegeben wird.

Im Jahr 2005 lag die Zahl der krankheitsbedingten Fehlzeiten von Vollzeitbeschäftigten zwischen rd. 25 Tagen in Schweden und weniger als 1 Tag in Griechenland (Abb. HE4.1). Gleich hinter Schweden verzeichneten die Vollzeitbeschäftigten in Finnland und Norwegen Fehlzeiten von 17 bzw. 20 Tagen, während die krankheitsbedingten Fehlzeiten in der Slowakischen Republik und den Vereinigten Staaten 5 Tage oder weniger betrugen. Frauen haben tendenziell längere krankheitsbedingte Fehlzeiten als Männer (zwischen 31 versäumten Arbeitstagen in Schweden und 5 Tagen in den Vereinigten Staaten, Tabelle HE4.2). Die krankheitsbedingten Fehlzeiten von weniger als einer Woche lagen in den europäischen Ländern durchschnittlich bei weniger als zwei Tagen; die Krankheitsdauer ist in den Ländern am längsten, in denen ganzwöchige Fehlzeiten häufiger sind (z.B. Finnland, Norwegen und Schweden), aber auch in den Niederlanden und im Vereinigten Königreich (wo sie zwischen drei und sechseinhalb Tagen liegen). Die krankheitsbedingten Fehlzeiten von einer Woche oder mehr haben je nach Land eine sehr unterschiedliche Dauer (wobei Schweden mit 17 Tagen Krankenurlaub an erster Stelle rangiert).

Seit 1995 haben sich die krankheitsbedingten Fehlzeiten unter den Vollzeitbeschäftigten in Belgien, Norwegen und Schweden deutlich erhöht, wogegen sie in den Niederlanden (im Zeitraum 1995-2005 von 18,8 auf 10 Tage) und in Portugal zurückgingen. Allgemein lässt der Krankenurlaub eine ausgeprägte jahreszeitliche Struktur erkennen, wobei seine Häufigkeit im Winter größer und im Sommer geringer ist. In einigen Ländern scheinen krankheitsbedingte Fehlzeiten prozyklisch zu sein (Belgien, Niederlande, Norwegen und Schweden), und dieses Schema könnte durch die stärkere Arbeitsbelastung während Konjunkturaufschwüngen und die Entlassung häufig abwesender Arbeitnehmer während Perioden der Konjunkturschwäche bedingt sein (Bonato und Lusinyan, 2004).

Krankheitsbedingte Fehlzeiten können zudem, wenn sie von langer Dauer sind, zum ständigen Rückzug aus dem Erwerbsleben führen. 2005 gaben in Finnland, Norwegen, Polen, Schweden, Ungarn und im Vereinigten Königreich zwischen $5 \%$ und $8 \%$ der Männer im Alter von 15 bis 64 Jahren an, auf Grund von Krankheit und ständiger Erwerbsunfähigkeit weder einer Beschäftigung nachzugehen noch auf Arbeitsuche zu sein; in Dänemark, Island und den Niederlanden wurden ähnliche Anteile für Frauen verzeichnet. Der Anteil der Befragten, die angeben, auf Grund von Krankheit und ständiger Erwerbsunfähigkeit nicht erwerbstätig zu sein, nimmt mit dem Alter zu, wobei die Rate für Personen im Alter von 50 bis 64 Jahren in mehreren Ländern bei $10 \%$ oder mehr liegt und in Polen (für Männer und Frauen) und Norwegen (nur für Frauen) $20 \%$ übersteigt.

Krankheitsbedingte Fehlzeiten und Ausscheiden aus dem Erwerbsleben stellen für die öffentlichen Finanzen und die Unternehmen eine Kostenbelastung dar, sie bedeuten eine Vergeudung menschlicher Ressourcen und Stress für die hiervon betroffenen Personen. Die finanziellen Leistungen wegen krankheitsbedingter Abwesenheit (öffentliche und private Pflichtleistungen) entsprachen $2001 \mathrm{im}$ OECD-Raum (ohne Zahlungen auf Grund von Erwerbsunfähigkeit) einem Anteil von rd. $0,8 \%$ des BIP, aber von über $2 \%$ in den Niederlanden und Norwegen. Die Reduzierung dieser Ausgaben ist heute in der Politik mehrerer OECD-Länder eine Priorität. Ein System gegenseitiger Verpflichtungen zwischen den Arbeitnehmern und den Versicherungsträgern in Verbindung mit effektiven Rehabilitationsdienstleistungen hat sich in vielen Ländern als eine wirksame Lösung erwiesen, um Personen, die hierzu körperlich in der Lage sind, eine schnelle Rückkehr ins Erwerbsleben zu ermöglichen (Rae, 2005; Bonato und Lusinyan, 2004).

\section{Statusindikatoren: Beschäftigung (SS1), Arbeitsunfälle (CO4).}

Reaktionsindikatoren: Öffentliche Sozialausgaben (EQ5), Gesamtsozialausgaben (EQ6), Langzeitpflegeausgaben (HE5). 
HE4.1 Eine hohe Zahl krankheitsbedingter Fehltage in den nordischen Ländern

Ausgewählte OECD-Länder im Jahr 2005, Vollzeitbeschäftigte

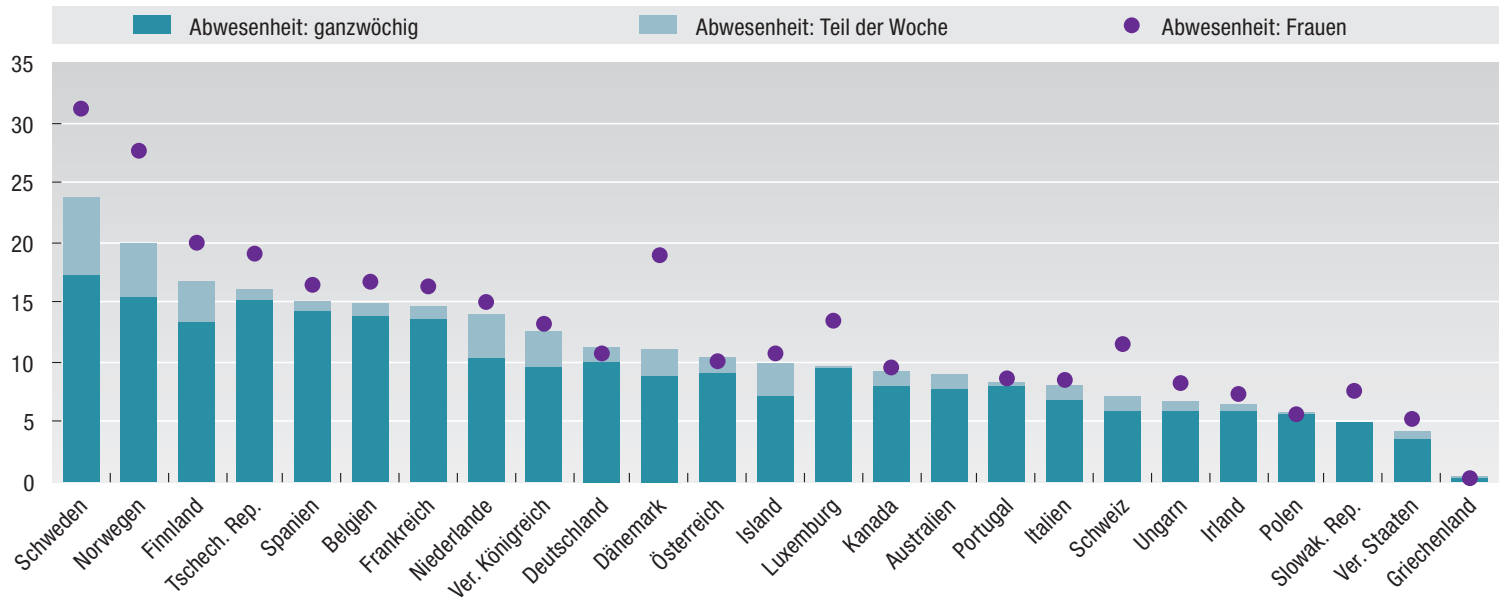

Anmerkung: Jahresdurchschnitt vierteljährlicher Schätzungen für die europäischen Länder; Durchschnitt monatlicher Schätzungen für Kanada und die Vereinigten Staaten. Für Australien und Kanada handelt es sich bei den krankheitsbedingten Fehlzeiten (ganze Woche oder Teil einer Woche) um Schätzwerte. Für die Vereinigten Staaten wurden die krankheitsbedingten Fehlzeiten von weniger als einer Woche geschätzt.

Quelle: Europäische Arbeitskräfteerhebung und Current Population Survey, 2004 National Health Survey für Australien und monatliche Arbeitsmarkterhebung für Kanada und die Vereinigten Staaten (CPS).

HE4.2 Krankheit und Erwerbsunfähigkeit sind wichtige Ursachen der Nichterwerbstätigkeit Ausgewählte OECD-Länder 2005, Bevölkerungsanteil jeder Gruppe

\begin{tabular}{|c|c|c|c|c|c|c|c|c|c|c|c|c|}
\hline & \multicolumn{4}{|c|}{ Erwerbsfähige Bevölkerung (15-64 Jahre) } & \multicolumn{4}{|c|}{ Erwachsene Bevölkerung (25-49 Jahre) } & \multicolumn{4}{|c|}{ Ältere (50-64 Jahre) } \\
\hline & \multicolumn{2}{|c|}{ Männer } & \multicolumn{2}{|c|}{ Frauen } & \multicolumn{2}{|c|}{ Männer } & \multicolumn{2}{|c|}{ Frauen } & \multicolumn{2}{|c|}{ Männer } & \multicolumn{2}{|c|}{ Frauen } \\
\hline & $\begin{array}{l}\text { Alle Nicht- } \\
\text { erwerbs- } \\
\text { tätigen }\end{array}$ & $\begin{array}{l}\text { Krankheit } \\
\text { und E- } \\
\text { werbs- } \\
\text { unfähigkeit }\end{array}$ & $\begin{array}{l}\text { Alle Nicht- } \\
\text { erwerbs- } \\
\text { tätigen }\end{array}$ & $\begin{array}{l}\text { Krankheit } \\
\text { und E- } \\
\text { werbs- } \\
\text { unfähigkeit }\end{array}$ & $\begin{array}{c}\text { Alle Nicht- } \\
\text { erwerbs- } \\
\text { tätigen }\end{array}$ & $\begin{array}{l}\text { Krankheit } \\
\text { und Er- } \\
\text { werbs- } \\
\text { unfähigkeit }\end{array}$ & $\begin{array}{l}\text { Alle Nicht- } \\
\text { erwerbs- } \\
\text { tätigen }\end{array}$ & $\begin{array}{l}\text { Krankheit } \\
\text { und Er- } \\
\text { werbs- } \\
\text { unfähigkeit }\end{array}$ & $\begin{array}{c}\text { Alle Nicht- } \\
\text { erwerbs- } \\
\text { tätigen }\end{array}$ & $\begin{array}{l}\text { Krankheit } \\
\text { und E- } \\
\text { werbs- } \\
\text { unfähigkeit }\end{array}$ & $\begin{array}{c}\text { Alle Nicht- } \\
\text { erwerbs- } \\
\text { tätigen }\end{array}$ & $\begin{array}{l}\text { Krankheit } \\
\text { und Er- } \\
\text { werbs- } \\
\text { unfähigkeit }\end{array}$ \\
\hline Australien & 16.9 & 2.6 & 30.9 & 2.9 & 9.0 & 2.3 & 25.4 & 2.4 & 32.9 & 6.5 & 53.8 & 7.4 \\
\hline Belgien & 27.3 & 3.6 & 42.3 & 3.1 & 6.6 & 2.5 & 22.1 & 3.1 & 43.9 & 8.1 & 65.4 & 5.1 \\
\hline Dänemark & 15.8 & 4.6 & 23.9 & 7.0 & 8.0 & 3.3 & 14.0 & 4.9 & 21.2 & 9.1 & 33.6 & 14.1 \\
\hline Deutschland & 21.0 & 2.4 & 34.9 & 2.2 & 6.6 & 1.7 & 20.8 & 1.6 & 31.3 & 4.9 & 48.4 & 4.3 \\
\hline Finnland & 21.6 & 7.4 & 25.9 & 6.0 & 8.1 & 3.8 & 14.7 & 3.0 & 32.4 & 16.9 & 33.8 & 13.8 \\
\hline Frankreich & 24.7 & 2.5 & 36.3 & 2.0 & 5.6 & 2.2 & 19.3 & 1.5 & 37.6 & 4.7 & 49.2 & 3.8 \\
\hline Giechenland & 20.9 & 2.2 & 45.9 & 1.3 & 4.0 & 1.8 & 29.0 & 1.0 & 30.0 & 3.8 & 66.1 & 2.4 \\
\hline Irland & 20.8 & 3.9 & 42.0 & 2.0 & 6.3 & 3.0 & 30.0 & 1.9 & 25.9 & 9.1 & 56.9 & 3.3 \\
\hline Island & 8.7 & 2.4 & 17.7 & 5.6 & 4.1 & 1.8 & 13.5 & 5.0 & 7.1 & 6.0 & 18.5 & 11.0 \\
\hline Italien & 24.9 & 2.4 & 49.6 & 3.0 & 7.0 & 1.7 & 33.7 & 2.1 & 41.6 & 4.7 & 69.7 & 5.8 \\
\hline Luxemburg & 25.2 & 3.3 & 45.7 & 2.3 & 3.9 & 1.6 & 28.7 & 0.9 & 39.8 & 9.0 & 65.6 & 6.6 \\
\hline Niederlande & 16.1 & 4.5 & 30.8 & 7.4 & 4.3 & 2.5 & 19.0 & 5.3 & 29.7 & 11.1 & 53.8 & 15.8 \\
\hline Norwegen & 18.2 & 7.0 & 24.5 & 9.0 & 9.2 & 4.9 & 16.4 & 6.0 & 21.7 & 15.0 & 31.8 & 20.3 \\
\hline Österreich & 22.9 & 2.2 & 36.5 & 1.8 & 7.4 & 1.7 & 19.2 & 1.6 & 45.7 & 4.1 & 63.6 & 3.0 \\
\hline Polen & 30.1 & 8.1 & 42.4 & 7.8 & 8.2 & 4.9 & 19.2 & 4.3 & 47.1 & 21.6 & 64.4 & 20.5 \\
\hline Portugal & 20.9 & 2.8 & 33.3 & 3.3 & 6.5 & 2.6 & 16.6 & 2.1 & 27.4 & 4.4 & 47.1 & 7.5 \\
\hline Schweden & 20.2 & 6.2 & 24.5 & 8.3 & 8.4 & 4.2 & 14.0 & 4.9 & 20.1 & 12.9 & 25.1 & 18.2 \\
\hline Schweiz & 12.0 & 2.7 & 26.1 & 2.5 & 3.9 & 1.9 & 18.7 & 1.7 & 15.6 & 5.8 & 36.0 & 5.5 \\
\hline Slowak. Rep. & 23.5 & 4.9 & 37.0 & 4.1 & 4.8 & 3.7 & 14.5 & 3.5 & 32.1 & 12.3 & 58.1 & 8.8 \\
\hline Spanien & 19.8 & 3.9 & 43.4 & 2.7 & 6.9 & 2.9 & 29.1 & 2.0 & 27.9 & 8.7 & 64.5 & 5.8 \\
\hline Tschech. Rep. & 22.4 & 4.5 & 37.9 & 4.4 & 4.2 & 2.9 & 19.8 & 3.3 & 28.3 & 10.1 & 48.4 & 8.6 \\
\hline Ungarn & 32.9 & 5.4 & 46.4 & 5.5 & 12.5 & 3.9 & 28.8 & 4.2 & 47.1 & 11.7 & 58.4 & 10.6 \\
\hline Ver. Königreich & 18.4 & 6.7 & 31.6 & 6.3 & 7.5 & 4.8 & 22.2 & 4.8 & 25.6 & 13.9 & 42.0 & 12.5 \\
\hline Ver. Staaten & 19.6 & 4.7 & 31.9 & 4.6 & 8.7 & 3.9 & 24.5 & 3.8 & 24.0 & 9.3 & 36.0 & 8.9 \\
\hline OECD24 & 21.0 & 4.2 & 35.0 & 4.4 & 6.7 & 2.9 & 21.4 & 3.1 & 30.7 & 9.3 & 49.6 & 9.3 \\
\hline
\end{tabular}

Quelle: Europäische Arbeitskräfteerhebung, September Labour Force Survey für Australien und Current Population Survey für die Vereinigten Staaten.

Stat Link 2 http://dx.doi.org/10.1787/7248471572302

Weitere Informationen — Bonato, L. und L. Lusinyan (2004), “Work Absence in Europe”, IMF Working Paper, European Department, Washington DC. - OECD (2004), “Clocking in (and out): several facets of working time”, Kapitel 1 in OECD Employment Outlook, Paris. Rae, D. (2005), "How to Reduce Sickness Absences in Sweden: Iessons from international experience", OECD Economics Department Working Paper, No. 442, Paris. 


\section{Definition und Messung}

Die Langzeitpflege umfasst eine ganze Reihe von Dienstleistungen für körperlich oder geistig eingeschränkte Personen, die Hilfe bei den Verrichtungen des täglichen Lebens benötigen. Zu diesen Verrichtungen zählen Waschen, Anziehen, Essen, Hilfe beim Hinlegen/Hinsetzen und Aufstehen in und aus Bett und Sessel, bei der Fortbewegung sowie bei der Toilettenbenutzung. Diese persönlichen Pflegeleistungen werden häufig in Kombination mit medizinischen Grundleistungen wie Wundbehandlung, Schmerzlinderung, Verabreichung von Medikamenten, Gesundheitsüberwachung, Vorbeugung, Rehabilitation oder Leistungen palliativer Pflege erbracht.

Die Langzeitpflege kann daheim oder in Einrichtungen verschiedener Art erbracht werden, z.B. in Pflegeheimen und Langzeitkliniken. Da in den letzten 15 Jahren in vielen OECD-Ländern neue Formen häuslicher Pflege für ältere Menschen entstanden sind, wird es immer schwieriger, sich auf eine Datenaufschlüsselung zu stützen, die allein zwischen häuslicher und institutioneller Pflege unterscheidet. Auf internationaler Ebene wird das Problem durch die Tatsache erschwert, dass mit ein und demselben Begriff Einrichtungen bezeichnet werden können, die sich relativ stark von denen unterscheiden, die in einem anderen Land unter demselben Begriff zusammengefasst sind. In diesem Abschnitt ist eine Langzeitpflegeeinrichtung als ein Ort gemeinschaftlichen Wohnens definiert, an dem Pflege und Unterkunft aus einer Hand kommen. Soweit nicht anders angegeben, handelt es sich hier sowohl um öffentliche als auch um private Einrichtungen. Die Daten über häusliche Pflegeleistungen beziehen sich nur auf solche, die entgeltlich erbracht werden (d.h. in privaten Haushalten unentgeltlich erbrachte Leistungen bleiben unberücksichtigt). Im Allgemeinen beziehen sich die Daten auf Personen ab 65 Jahre, doch im Falle der Slowakischen Republik, der Tschechischen Republik und Ungarns schließen sie die Langzeitpflegebedürftigen jeden Alters mit ein, so dass die Schätzungen gegenüber den von anderen Ländern zur Verfügung gestellten Daten überhöht sind.

Die Erbringung und Finanzierung von Langzeitpflegeleistungen ist insofern enorm wichtig, als die Bevölkerungsalterung Wirkung zu zeigen beginnt und Frauen auf Grund ihrer zunehmenden Erwerbsbeteiligung weniger in der Lage und bereit sind, die Pflege anderer Familienmitglieder zu übernehmen. Im Jahr 2004 (oder dem letzten verfügbaren Jahr) lebten in den meisten OECD-Ländern zwischen $3 \%$ und $6 \%$ der Personen im Alter von 65 Jahren oder darüber in Langzeitpflegeeinrichtungen (Abb. HE5.1). Der Anteil älterer Menschen, die in entsprechenden Einrichtungen Leistungen der Langzeitpflege in Anspruch nehmen, reichte von weniger als $1 \%$ in Korea bis 7,5\% in Schweden.

In den letzten zehn Jahren ist der prozentuale Anteil in Langzeitpflegeeinrichtungen lebender älterer Menschen in vielen Ländern gesunken, was zumindest teilweise die Präferenz der meisten älteren Menschen widerspiegelt, Pflegeleistungen nach Möglichkeit im häuslichen Bereich zu erhalten. In den Niederlanden zum Beispiel sank der Anteil in Langzeitpflegeeinrichtungen lebender älterer Menschen im Zeitraum 1995-2004 von 7,6\% auf 5,6\% und in Schweden von $8,8 \%$ auf $7,5 \%$. In den Vereinigten Staaten führte die Entwicklung von Alternativlösungen zur Versorgung älterer Menschen in Pflegeeinrichtungen, wie z.B. neuartige Wohneinrichtungen für ältere Menschen mit nur leichter Behinderung, in den letzten zehn Jahren ebenfalls zu einer Reduzierung des Anteils der in Pflegeeinrichtungen lebenden Personen, doch hatte dies zur Folge, dass das Alter der Bewohner von Langzeitpflegeeinrichtungen und der Grad ihrer Behinderung jetzt höher sind als in der Vergangenheit. Dagegen ist der Anteil der in Langzeitpflegeeinrichtungen lebenden älteren Menschen in Deutschland und Österreich in den letzten zehn Jahren wie auch in Luxemburg und Japan (zwischen 2000 und 2004) gestiegen. In diesen Ländern erfolgte dieser Anstieg parallel zur Einführung einer Pflegeversicherung, so dass sich die Kosten der direkt von Einzelpersonen getragenen Langzeitpflegeleistungen reduzierten (1993 in Österreich, 1995 in Deutschland, 1998 in Luxemburg und 2000 in Japan).

Um Menschen mehr Wahlmöglichkeiten bei ihren Pflegeentscheidungen zu lassen und die Erbringung von Pflegeleistungen im häuslichen Bereich zu fördern, wurden von mehreren Ländern Programme eingeführt, die Vergünstigungen für Personen einräumen, die Leistungen im Rahmen der häuslichen Pflege in Anspruch nehmen. Diese Programme sind in den einzelnen Ländern unterschiedlich ausgestaltet. Im Großen und Ganzen ist zwischen zwei Programmkategorien zu unterscheiden. Die erste Kategorie bezieht sich auf Programme, bei denen Pflegebedürftige Zahlungen erhalten, die sie nach Belieben für die Inanspruchnahme geeigneter Pflegeleistungen verwenden können. Bei der zweiten Kategorie handelt es sich um Programme, bei denen pflegende Angehörige Einkommensstützung erhalten. Auf Grund des derzeitigen prioritären Politikanliegens, die Versorgung älterer behinderter Menschen (möglichst) im eigenen häuslichen Umfeld zu fördern, hat sich der Anteil der Menschen im Alter von 65 Jahren, die in der einen oder anderen Art formelle (bezahlte) häusliche Pflegeleistungen erhalten, in Dänemark, Norwegen und Schweden in den letzten zehn Jahren sowie in Japan, Luxemburg und Österreich in den letzten fünf bis zehn Jahren erhöht (Abb. HE5.2). In den meisten OECD-Ländern erhalten heute zwischen 5\% und 10\% der älteren Menschen eine Art formeller Leistungen häuslicher Langzeitpflege.

\footnotetext{
Statusindikatoren: Lebenserwartung (HE1).

Reaktionsindikatoren: Öffentliche Sozialausgaben (EQ5), Gesamtsozialausgaben (EQ6), Langzeitpflegeausgaben (HE5).
} 


\section{HE5.1 In den meisten OECD-Ländern lebt ein geringerer Anteil älterer Menschen in Pflegeeinrichtungen}

Anteil der in Pflegeeinrichtungen lebenden Menschen im Alter von 65 Jahren und darüber,

in Prozent der Personen im Alter von 65 Jahren und darüber, 1995 und 2004

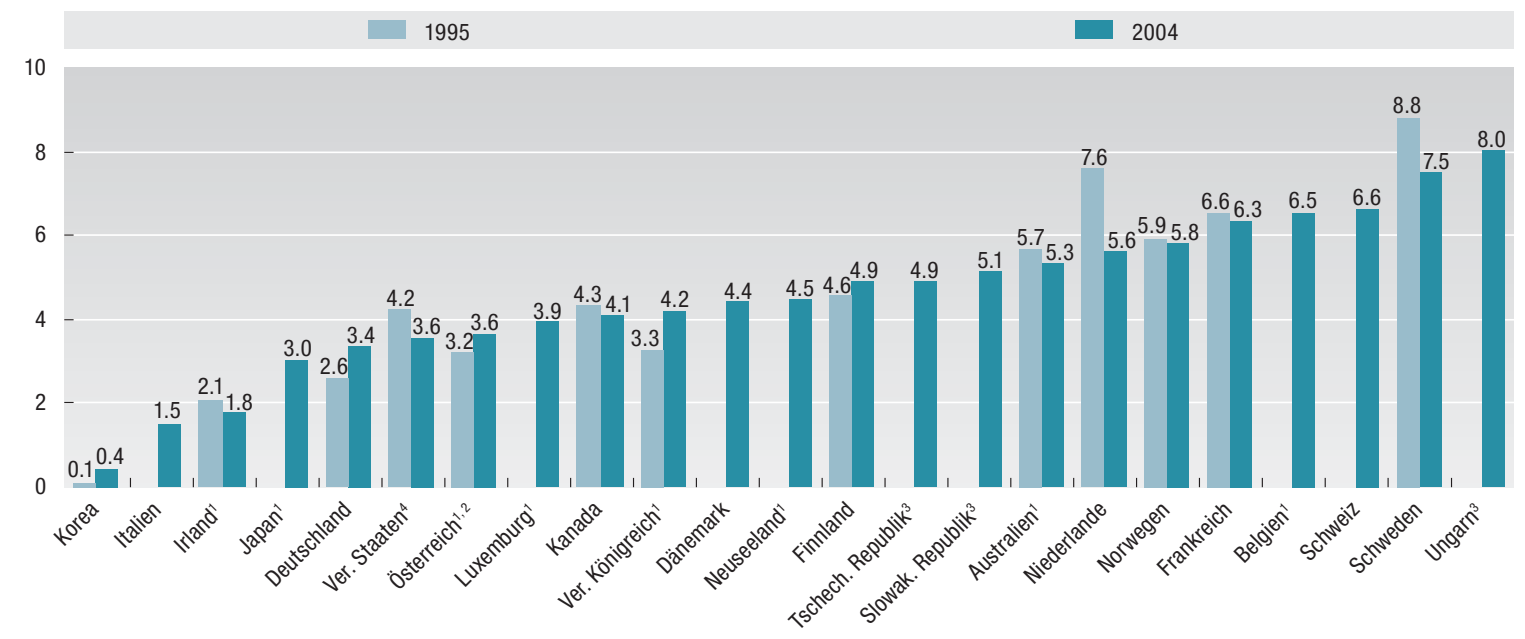

Anmerkung: Die Länder sind in aufsteigender Reihenfolge von links nach rechts entsprechend ihrem Anteil älterer Menschen angegeben, die im letzten verfügbaren Jahr in Pflegeheimen versorgt wurden. Die Daten für die Vorperiode beziehen sich für Australien und Deutschland auf 1996, für Österreich und das Vereinigte Königreich auf 1997, für Frankreich auf 1994. Die Daten für den letzten Zeitraum beziehen sich für Kanada auf 2001, für Finnland, Frankreich, Italien, Norwegen, Österreich, die Schweiz, die Slowakische Republik, die Tschechische Republik und Ungarn auf 2003.

1. Die Daten beziehen sich lediglich auf Personen, die mit öffentlichen Mitteln finanzierte Leistungen der Langzeitpflege in Einrichtungen erhalten.

2. Die Daten beziehen sich auf die Bevölkerung im Alter von 60 Jahren und darüber.

3. Die Daten beziehen sich auf die Bevölkerung jeden Alters.

4. Bei den US-Daten für 2004 bleiben Personen unbekannten Alters unberücksichtigt (etwa 1,5\% der Pflegeheimbewohner im Jahr 2004).

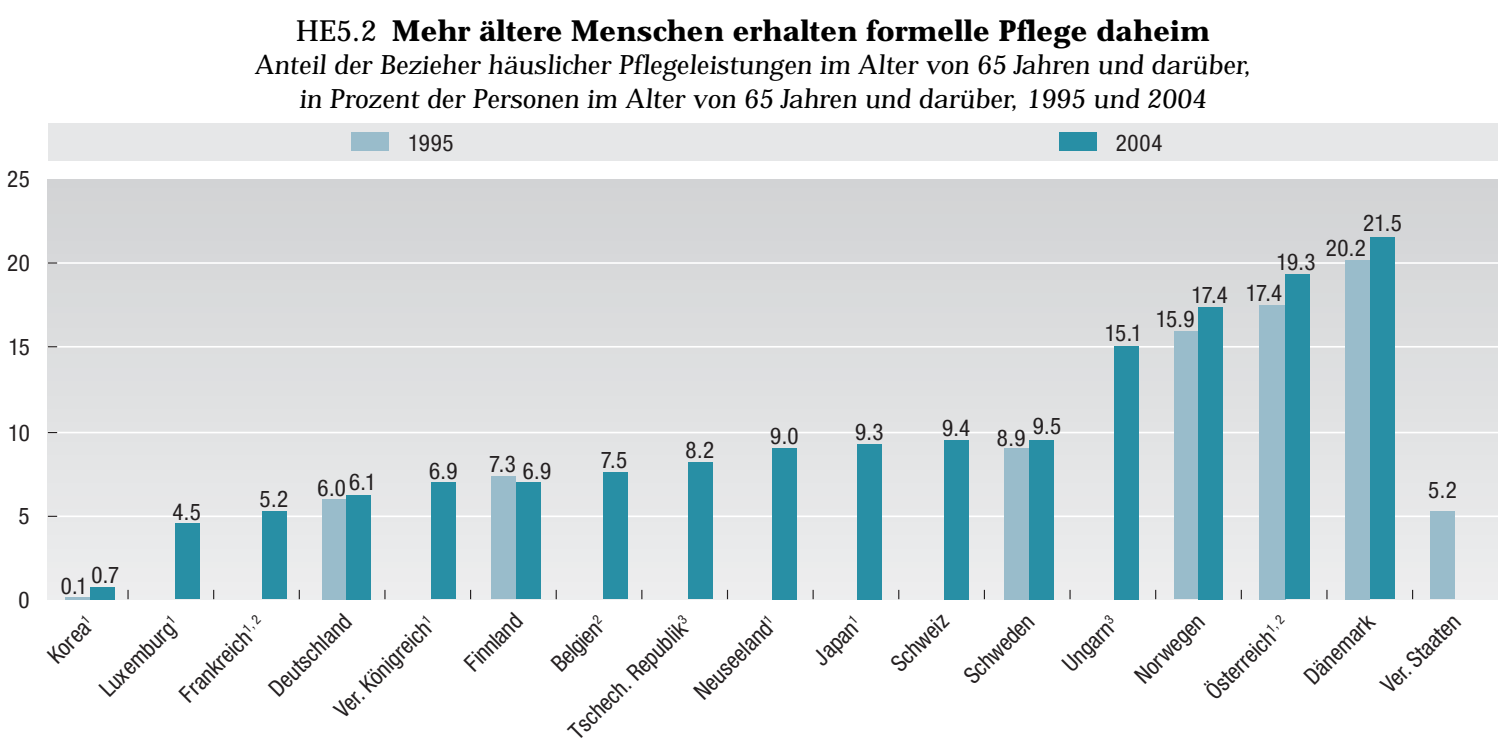

Anmerkung: Die Daten für Deutschland beziehen sich auf das Jahr 1996, für Österreich auf das Jahr 1997 und für Frankreich auf das Jahr 1994 (anstatt 1995). Die Daten für Finnland, Frankreich, Italien, Norwegen, Österreich, die Schweiz, die Slowakische Republik, die Tschechische Republik und Ungarn beziehen sich auf das Jahr 2003 (anstatt 2004).

1. Die Daten für Irland, Japan, Luxemburg, Neuseeland, Österreich und das Vereinigte Königreich beziehen sich lediglich auf Personen, die mit öffentlichen Mitteln finanzierte häusliche Langzeitpflege erhalten, was dazu führt, dass die für diese Länder angegebenen Anteile unterschätzt sind.

2. Die Daten für die Empfänger von Langzeitpflegeleistungen beziehen sich in Belgien, Frankreich und Österreich auf die Bevölkerung im Alter von 60 Jahren und darüber. Daraus ergibt sich eine überhöhte Schätzung (auf Grund der Tatsache, dass der Nenner zur Errechnung der Anteile nur die Bevölkerung im Alter von 65 Jahren und darüber einschließt).

3. Die Daten über die Empfänger von häuslichen Langzeitpflegeleistungen sind für die Tschechische Republik und Ungarn nur für die Bevölkerung jeden Alters verfügbar, darunter auch die unter 65-Jährigen.

Quelle: OECD (2006), OECD-Gesundheitsdaten 2006, Paris (www.oecd.org/health/healthdata).

StatLink 2 http://dx.doi.org/10.1787/013105642736

Weitere Informationen — Lunsgaard, J. (2005), "Consumer Direction and Choice in Long-Term Care for Older Persons", OECD Health Working Paper, No. 20, Paris. — OECD (2005), Long-Term Care for Older People, Paris. 


\section{Definition und Messung}

Gesundheitliche Ungleichheit lässt sich auf verschiedene Art und Weise beschreiben. In diesem Abschnitt werden zwei Indikatoren vorgestellt, und beide beziehen sich auf die Mortalität (anstatt auf die Morbidität). Der erste Indikator misst die Differenzen beim Sterbealter - oder aber der Lebenslänge - von Personen in verschiedenen OECD-Ländern nach der Definition von Edwards und Tuljapurkar (2005). Die hier verwendete spezifische Messgröße der Differenzen im Sterbealter ist die Standardabweichung aller Sterbefälle im Alter über zehn Jahren. Die Hauptvorteile dieses Indikators sind seine Einfachheit und die Tatsache, dass er eine direkte Messung der Ungleichheiten zwischen einzelnen Personen in Bezug auf die Gesundheit ermöglicht. Dieser Indikator basiert auf Daten der Human Mortality Database und ist für die meisten OECD- und Nicht-OECD-Länder als Zeitreihe sowohl für die Gesamtbevölkerung als auch geschlechtsspezifisch verfügbar.

Der zweite Indikator bezieht sich auf die durchschnittliche Mortalitätsrate von Personen mit unterschiedlichen Merkmalen. Diese Unterschiede zwischen einzelnen Gruppen Iassen sich in absoluten Zahlen (Differenz zwischen der Lebenserwartung verschiedener Gruppen) und relativ (Verhältnis zwischen verschiedenen Lebenserwartungen) ausdrücken. Die meisten Studien über gesundheitliche Ungleichheit zwischen einzelnen Gruppen basieren auf parallelisierten Daten, d.h. auf der Verknüpfung von Daten aus Sterberegistern (über die Zahl der in einem bestimmten Zeitraum innerhalb von Bevölkerungsuntergruppen erfassten Sterbefälle) mit Volkszählungsdaten (über die Personenzahl innerhalb dieser Untergruppen). Solche Ungleichheiten wurden zwar für einzelne Länder im Rahmen mehrerer Studien dokumentiert, doch gibt es auf internationaler Ebene nur vereinzelte Befunde, und die verwendeten Messgrößen sind unterschiedlich im Hinblick auf die Merkmale der Personen (Bildung, Einkommen, Wohnort, ethnische Zugehörigkeit), die (oft auf ältere Personen begrenzte) Referenzpopulation, den geographischen Erfassungsbereich (oft ganz spezifische Städte eines Landes) und die Zahl der erfassten Jahre. Auf Grund dieser Unterschiede lässt sich der Grad dieser gesundheitlichen Ungleichheit nicht immer direkt zwischen den einzelnen Ländern vergleichen. Die hier vorgestellten Schätzungen sind von Mackenbach (2006) und beschränken sich auf die europäischen Länder.

Gesundheitliche Ungleichheit gibt es überall, nicht nur zwischen, sondern auch in den einzelnen Ländern. Abbildung HE6.1 zeigt den Trend eines Indikators der gesundheitlichen Ungleichheit - die Standardabweichung des Sterbealters nach dem zehnten Lebensjahr (ohne den in allen OECD-Ländern eingetretenen Rückgang der Kindersterblichkeit). Unter den OECD-Ländern waren diese Differenzen 2003 in den Vereinigten Staaten und Frankreich am größten, am geringsten in den Niederlanden und Schweden. Japan, dessen Ausgangsniveau 1960 nahe dem der Vereinigten Staaten lag, hatte bis Anfang der neunziger Jahre einen starken Rückgang zu verzeichnen, erlebt seither jedoch wieder einen Anstieg (Edwards und Tuljapurkar, 2005). Dagegen kam es in Dänemark, dessen niedriges Ausgangsniveau nahe dem schwedischen lag, seit 1990 zu einem starken Anstieg und anschließend zu einem Rückgang. Insgesamt gesehen ist dieser Indikator der gesundheitlichen Ungleichheit seit 1960 gegenüber früheren Zeiträumen nur wenig gesunken.

Die Differenzen zwischen einzelnen Ländern in Bezug auf die gesundheitliche Ungleichheit auf der Ebene von Einzelpersonen sind durch Unterschiede sowohl innerhalb der einzelnen Gruppen als auch zwischen den Gruppen bedingt. Personen mit niedrigerem Bildungsniveau und geringerem Einkommen bzw. Angehörige von Berufsgruppen mit geringerem Status sterben tendenziell jünger und weisen während ihres kürzeren Lebens eine höhere Prävalenz für verschiedene Gesundheitsprobleme auf (Mackenbach, 2006). Abbildung HE6.2 liefert Befunde für einen mit dem Qualifikationsniveau steigenden "sozialen Gradienten" der Lebenserwartung in England und Wales, wobei die Lebenserwartung beim Aufstieg vom nichtqualifizierten Arbeiter zum qualifizierten, vom Arbeiter zum Angestellten, vom unteren Büroangestellten zum höheren jeweils steigt. Auch die Unterschiede in Bezug auf die durchschnittliche Lebenserwartung verschiedener ethnischer Gruppen sind beachtlich; das Spektrum dieser Unterschiede reicht von 61/2Jahren Unterschied zwischen afro-amerikanischen und weißen Männern in den Vereinigten Staaten (2003) und zwischen registrierten Indianern (registered Indians) und Nichtindianern in Kanada (1998) bis zu 18 Jahren zwischen Aborigines und Torres-Strait-Insulanern und der übrigen nicht den Ureinwohnern Australiens zugehörenden Bevölkerung (1996-2000).

In europäischen Ländern haben generell weniger gebildete Personen im Durchschnitt eine um rd. 15\% geringere Lebenserwartung als Personen mit höherem Bildungsniveau (Abb. HE6.3). Diese Ungleichheiten sind bei Männern größer als bei Frauen und nehmen mit zunehmendem Alter ab. Im Zeitverlauf ist keine eindeutige Verringerung dieser gesundheitsspezifischen Unterschiede zwischen den Gruppen und in einigen europäischen Ländern sogar eine Vergrößerung festzustellen (Mackenbach, 2006). Doch selbst wenn die Ungleichheit zwischen den einzelnen Gruppen groß ist, erklärt sie doch nur einen Teil der zwischen den Ländern bestehenden Differenzen in Bezug auf die Streuung des individuellen Sterbealters, was darauf schließen lässt, dass andere Faktoren - z.B. in Bezug auf Generika und die vielen unterschiedlichen Lebensweisen - eine Rolle spielen (Edwards und Tuljapurkar, 2005).

Das Wissen um diese gesundheitlichen Unterschiede spiegelt sich in der Tatsache wider, dass mehrere europäische Länder öffentliche Gesundheitsstrategien oder andere Initiativen eingeleitet haben, um diese Diskrepanzen zu reduzieren (Mackenbach und Bakker, 2003). Auf internationaler Ebene haben die Weltgesundheitsorganisation und die Europäische Union in jüngster Zeit Ausschüsse für die Untersuchung der sozialen Bestimmungsfaktoren der Gesundheit gegründet.

Statusindikatoren: Lebenserwartung (HE1), Generationenmobilität (EQ4).

Reaktionsindikatoren: Langzeitpflegebedürftige (HE5). 


\section{HE6.1 Die Differenzen beim Sterbealter sind in den Vereinigten Staaten und Frankreich am größten}

Standardabw eichung des Sterbealters (A Iter > 10 Jahre), Männer und Frauen insgesamt
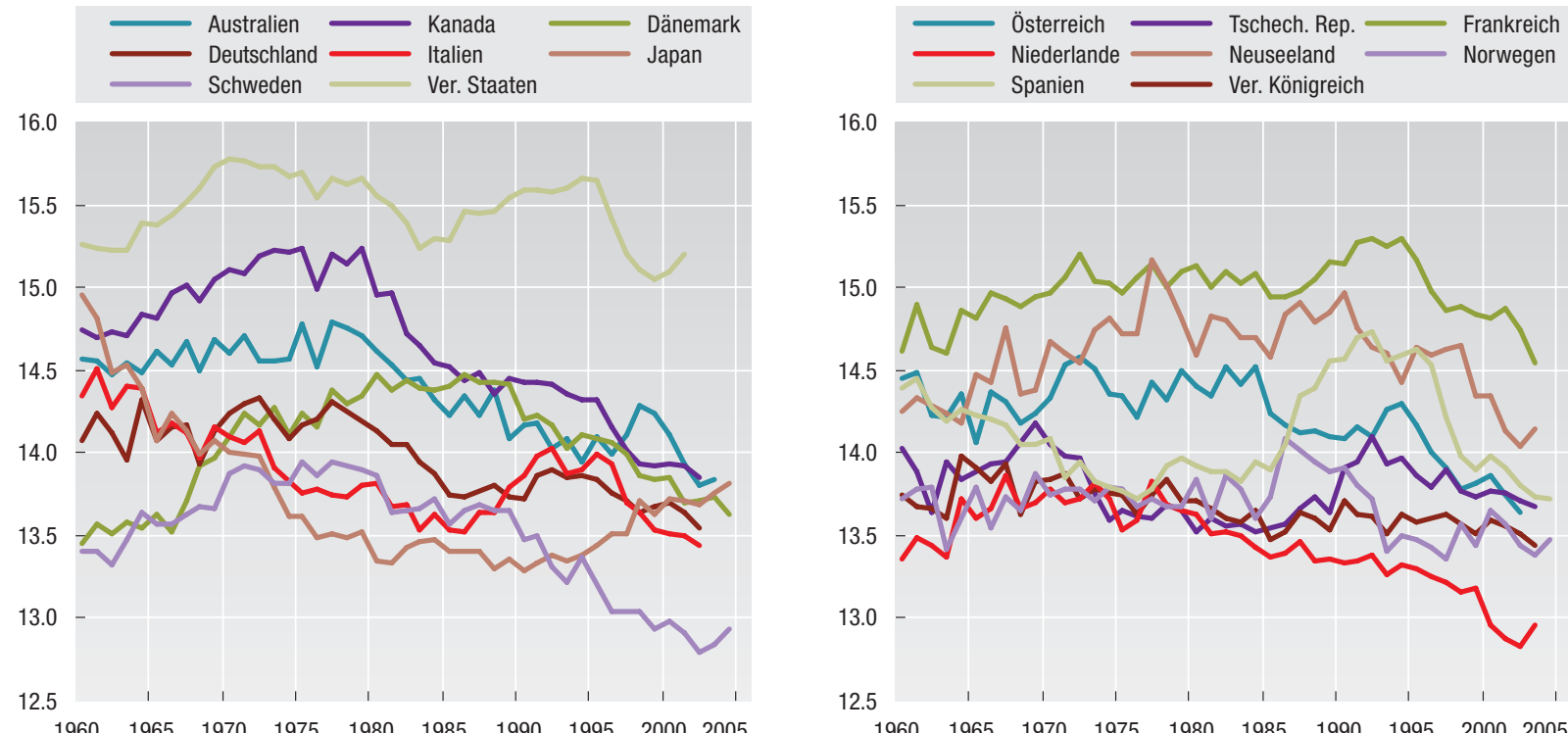

$\begin{array}{llllllllll}1960 & 1965 & 1970 & 1975 & 1980 & 1985 & 1990 & 1995 & 2000 & 2005\end{array}$

Quelle: R.D. Edwards und S. Tuljapurkar (2005), “Inequality in Life Spans and a New Perspective on Mortality Convergence Across Industrialised Countries", Population and Development Review, Vol. 34, Nr. 4, Dezember.

HE6.2 Ansteigende soziale Gradiente der Lebenserwartung nach Qualifikationsniveau Lebenserwartung bei der Geburt nach Qualifikationsniveau in England und Wales

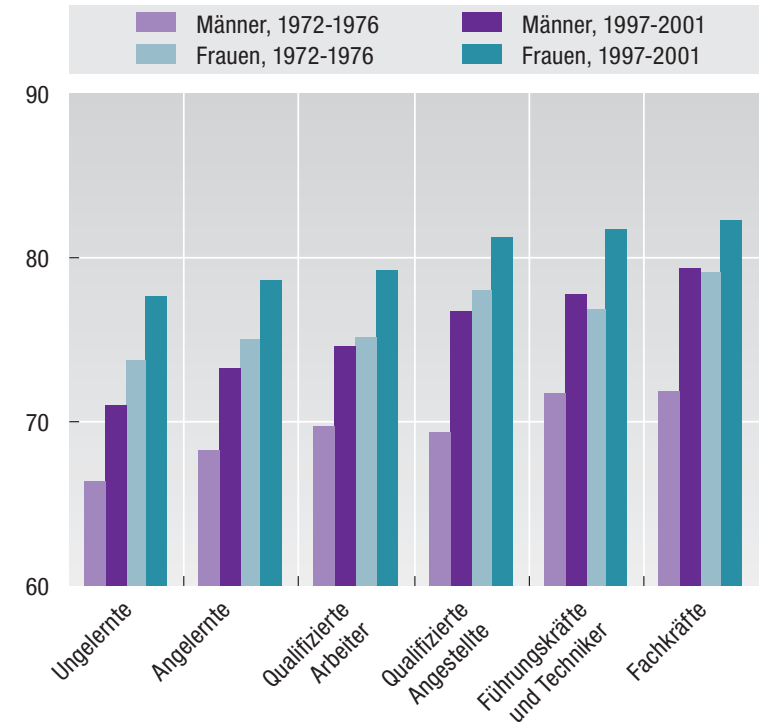

Quelle: National Statistics, "Trends in Life Expectancy by Social Class, 1972-2001", Statistisches Amt, Vereinigtes Königreich.

\section{HE6.3 Höhere Mortalitätsrate von Personen mit niedrigerem Bildungsniveau}

Verhältnis M ortalitätsraten von Personen mit niedrigerem/ höherem Bildungsniveau in ausgewählten europäischen Ländern

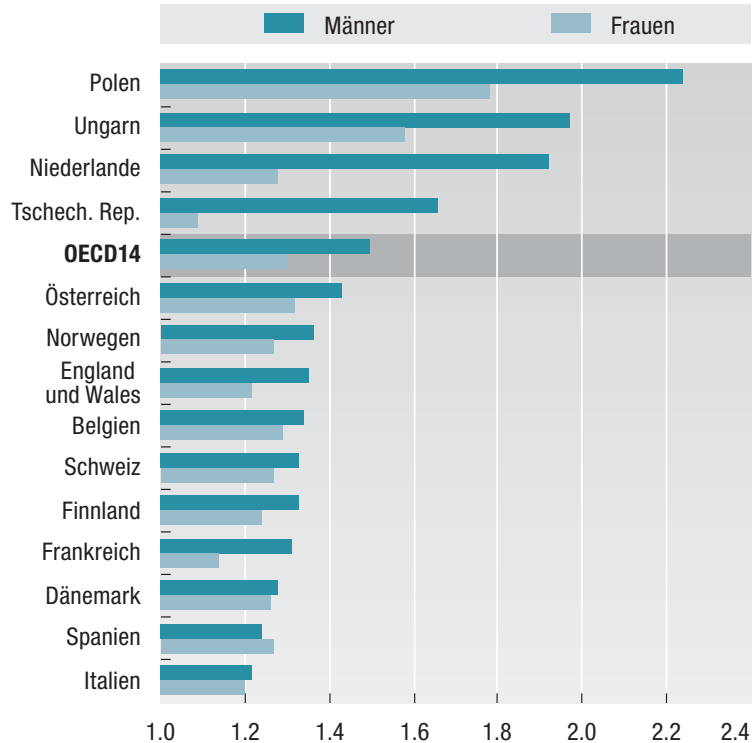

Anmerkung: Die Länder sind in absteigender Reihenfolge der relativen Ungleichheit zwischen Männern angegeben. Die relative Ungleichheit wird gemessen am Verhältnis der Mortalitätsrate der Gruppe mit niedrigerem gegenüber der mit höherem Bildungsniveau. Die nationalen Schätzungen können sich auf Personen unterschiedlichen Alters beziehen.

Quelle: J.P. Mackenbach (2006) “Health Inequalities: Europe in ProfiIe", EU-Vorsitz des Vereinigten Königreichs, Februar.

$$
\text { StatLink } 2 \text { http://dx.doi.org/10.1787/564263058115 }
$$

Weitere Informationen —Edwards, R.D. und S. Tuljapurkar (2005), “Inequality in Life Spans and a New Perspective on Mortality Convergence Across Industrialised Countries", Population and Development Review, Vol. 34, No. 4, Dezember. $\square$ Macken bach, J.P. und M.J. Bakker (2003), “Tackling Socioeconomic Inequalities in Health: analysis of European experiences", The Lancet, Oktober. 




\section{Definition und Messung}

Die Wahlbeteiligung ist ein Indikator für die Beteiligung der Bevölkerung am Leben ihres Gemeinwesens. Der hier verwendete Indikator zur Messung der Beteiligung des Einzelnen am Wahlprozess ist die "Wahlbeteiligung", d.h. die Anzahl der Personen, die bei einer Wahl eine Stimme abgeben, ausgedrückt als Anteil der Bevölkerung im Wahlalter - d.h. generell die Bevölkerung über 18 Jahre -, wie sie den amtlichen Statistiken der Mitgliedsländer zu entnehmen ist. In den einzelnen Ländern finden entsprechend ihrer institutionellen und ihrer jeweiligen hoheitlichen Struktur verschiedene Arten von Wahlen statt. In dieser Analyse werden die Wahlen betrachtet, die in jedem Land die größte Wählerschaft mobilisieren: Präsidentschaftswahlen in Finnland, Frankreich, Korea, Mexiko, Polen und den Vereinigten Staaten sowie Parlamentswahlen in den anderen OECD-Ländern. Die Daten über die Wahlbeteiligung sind der vom Institut für Demokratie und Wahlhilfe (IDEA) eingerichteten internationalen Datenbank entnommen.

Der folgende Beitrag enthält auch Daten über die Wahlbeteiligung nach ausgewählten soziodemographischen Merkmalen. Diese Daten, die im Rahmen von Personenbefragungen nach wichtigen Wahlen erhoben werden, beruhen auf Ergebnissen der Vergleichenden Untersuchung von Wahlsystemen (CSES), einem internationalen Forschungsprogramm, das vergleichbare Wahldaten sammelt. Schätzungen der gesamten Wahlbeteiligung auf der Basis dieser Erhebungen können von den in Abbildung CO1.1 dargestellten Schätzwerten abweichen, die auf Verwaltungsdaten fußen.

Eine hohe Wahlbeteiligung ist ein Zeichen dafür, dass das politische System eines Landes ein hohes Maß an Legitimität genießt. Obwohl eine geringe Wahlbeteiligung prinzipiell als Ausdruck für den Grad der Zufriedenheit der Bevölkerung mit der politischen Führung ihres Landes gewertet werden könnte, bedeutet sie gleichzeitig, dass das politische System des betreffenden Landes nur den Willen einer begrenzten Zahl von Personen widerspiegelt. Nachdem die Wahlbeteiligung mehrere Jahrzehnte gestiegen ist, war sie in den meisten OECD-Ländern und auch im Durchschnitt in den vergangenen zehn Jahren rückläufig (Abb. CO1.1). Die Wahlbeteiligung weist zwischen den OECD-Ländern enorme Unterschiede auf, sie liegt in der Schweiz, in Polen, Kanada, den Vereinigten Staaten, Luxemburg, Ungarn und dem Vereinigten Königreich bei unter $60 \%$ und in Spanien, Dänemark, Italien, Korea, Belgien und Island bei über $80 \%$. Obgleich davon ausgegangen werden kann, dass Wahlpflicht die Wahlbeteiligung beeinflusst, scheint sie die zwischen den Ländern beobachteten Unterschiede nur bedingt zu erklären. Wahlpflicht besteht in Australien, Österreich (für Bundespräsidentenwahlen), Belgien, Griechenland, Luxemburg, Mexiko (nicht durchgesetzt), (Teile der) Schweiz und der Türkei (nicht durchgesetzt), und viele dieser Länder verzeichnen ebenfalls geringe Wahlbeteiligungen.

Die soziodemographischen Merkmale des Einzelnen - Alter, Bildungsniveau, Einkommen - haben einen bedeutenden Einfluss auf die Wahrscheinlichkeit seiner Wahlbeteiligung (Tabelle CO1.2). Im Durchschnitt der OECD-Länder ist die Wahlbeteiligung bei Frauen etwas niedriger als bei Männern, wobei diesbezüglich in Ungarn, Mexiko, Belgien, der Schweiz und Irland größere Unterschiede bestehen, umgekehrt die Wahlbeteiligung der Frauen in neun OECDLändern, darunter die Vereinigten Staaten, das Vereinigte Königreich, Australien und Frankreich, aber höher ist. Die Wahlbeteiligung nimmt mit dem Alter stetig zu, wobei sie unter jungen Menschen im Durchschnitt 20 Prozentpunkte niedriger ist als in der Gruppe der Personen ab 65 Jahre. In etwa der Hälfte der OECD-Länder verzeichnen ältere Personen die höchste Wahlbeteiligung von allen untersuchten Bevölkerungsgruppen. Die höhere Wahlbeteiligung älterer Personen bei landesweiten Wahlen kann sich auch auf den politischen Prozess auswirken und die Gefahr erhöhen, dass Regierungen, die Kürzungen bei jenen Sozialprogrammen vorsehen, die älteren Menschen unverhältnismäßig stark zugute kommen, bei den Wahlen abgestraft werden.

Das Bildungsniveau ist ein weiterer entscheidender Bestimmungsfaktor der Wahlbeteiligung. Im Durchschnitt ist die Wahlbeteiligung unter den Personen mit dem höchsten Bildungsabschluss um 12 Prozentpunkte höher als unter denjenigen mit dem niedrigsten Bildungsabschluss, wobei die Unterschiede in Frankreich, Portugal, der Schweiz und der Tschechischen Republik größer sind. Im Vergleich zu den Erwerbstätigen ist die Wahlbeteiligung auch unter den Rentnern hoch, während sie unter "Hausfrauen" und in stärkerem Maß unter Studenten, Arbeitslosen und Personen mit Behinderungen geringer ist. Schließlich steigt die Wahlbeteiligung mit dem Einkommen, dieser Effekt lässt aber ganz oben in der Verteilung nach. Alles in allem verzeichnen Personen im unteren Quintil der Einkommensverteilung Wahlbeteiligungsquoten, die um $12 \%$ niedriger sind als im obersten Quintil, mit einem Abstand von $20 \%$ oder mehr in Kanada, Finnland, Ungarn, Korea, Mexiko, Norwegen, Portugal, dem Vereinigten Königreich und den Vereinigten Staaten. Zwischen den Beziehern mittlerer und hoher Einkommen sind die Unterschiede in der Wahlbeteiligung geringer, wobei die Beteiligungsquoten in mehreren Ländern in der erstgenannten Gruppe höher sind als in der letztgenannten.

Statusindikatoren: Vertrauen in die politischen Institutionen (CO6), Lebenszufriedenheit (CO7). 


\section{CO1.1 Niedrigere Wahlbeteiligung in den meisten OECD-Ländern in den vergangenen zehn Jahren}

Zahl der Personen, die bei einer Wahl eine Stimme abgeben, als Anteil der Bevölkerung im Wahlalter
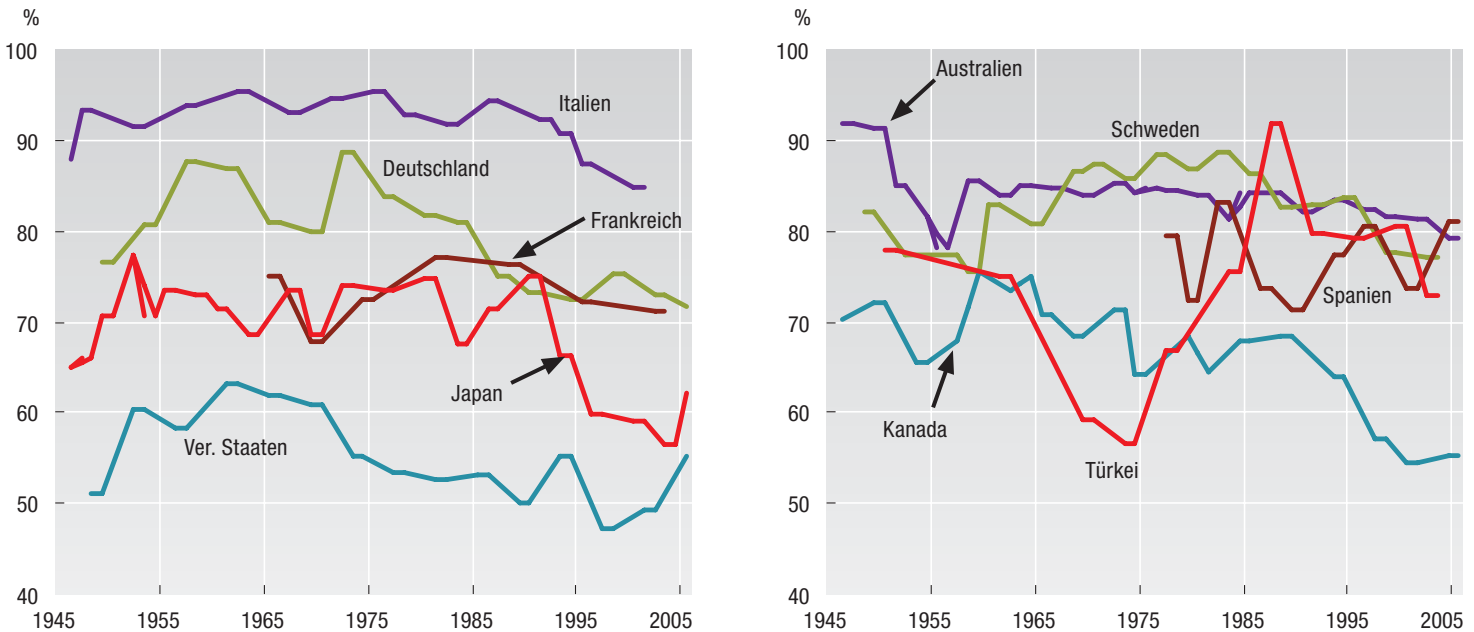

Anmerkung: Für einige Länder sind die Wahlbeteiligungsquoten bei den jüngsten Wahlen berechnet worden als das Verhältnis zwischen den Personen, die laut IDEA-Datenbank eine Stimme abgegeben haben, und der Bevölkerung im Wahlalter gemäß OECD-Schätzung. Quelle: Internationales Institut für Demokratie und Wahlhilfe (IDEA), Stockholm.

\section{CO1.2 Geringere Wahlneigung bei jüngeren, weniger gebildeten und einkommensschwächeren Personen}

Wahlbeteiligung nach ausgewählten sozioökonomischen M erkmalen, in Relation zu verschiedenen Bevölkerungsgruppen

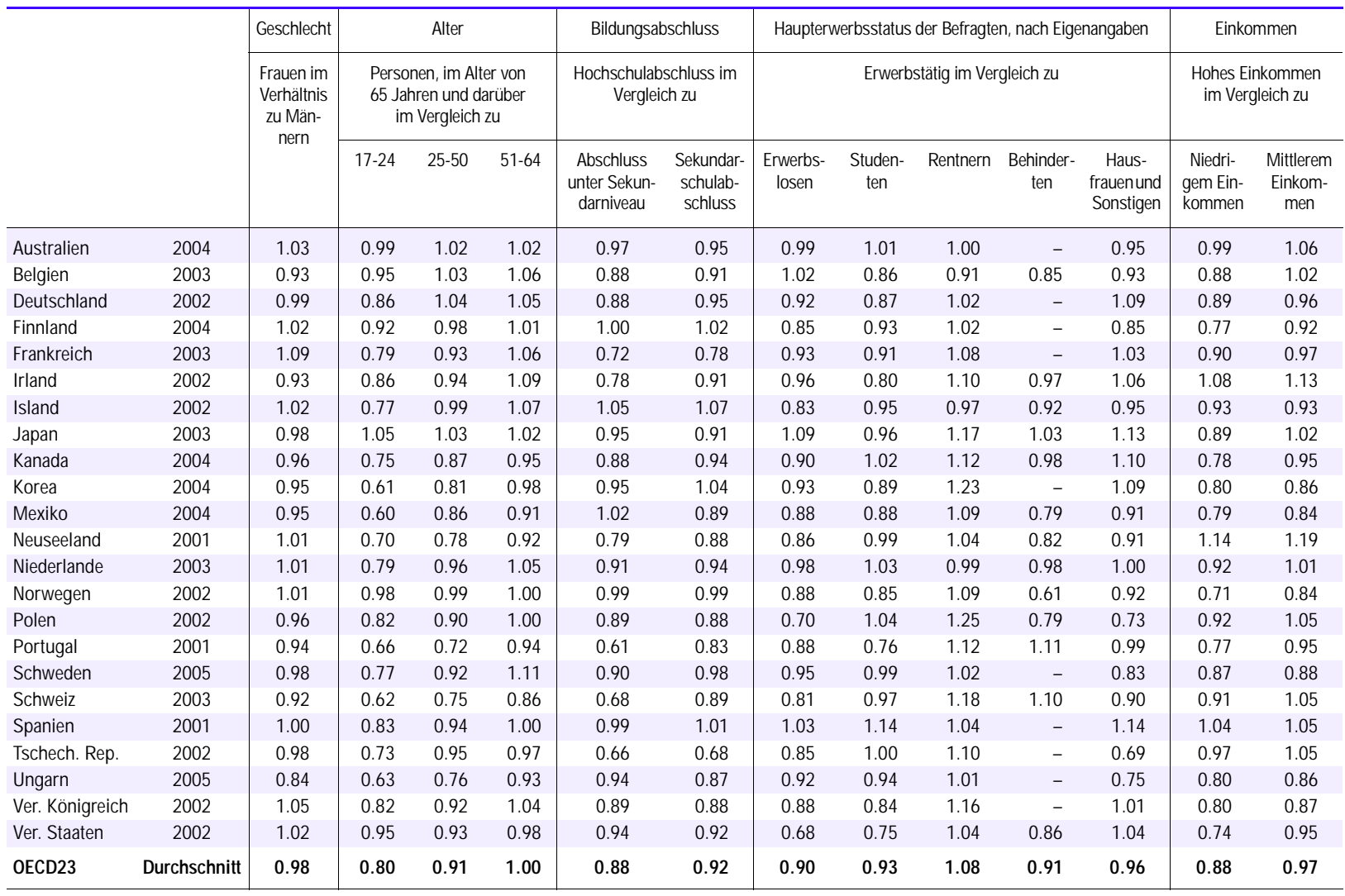

A nmerkung: Einfacher Durchschnitt für die aufgeführten Länder. Die Schätzungen der gesamten Wahlbeteiligung anhand dieser Erhebungsdaten können etwas von den auf Verwaltungsdaten fußenden Schätzungen in Abb. CO1.1 abweichen.

Quelle: Modul 2 der Vergleichenden Untersuchung von Wahlsystemen (CSES).

Weitere Informationen $\square$ López Pintor, R. und M. Gratschev (2002), Voter Turnout since 1945 - A Global Report, International Institute for Democracy and Electoral Assistance (IDEA), Stockholm. 


\section{Definition und Messung}

Verbrechen verursachen nicht nur großes Leid für die Opfer und ihre Familien, sie sind auch ein Ausdruck der extremen Ausgrenzung einiger Personen von der Mehrheitsgesellschaft. Mit der Kriminalität entstehen der Gesellschaft durch die Inhaftierung auch generell hohe Kosten, die normalerweise unter Hinweis auf drei "Bedürfnisse" der Gesellschaft gerechtfertigt werden: die Täter zu bestrafen, andere von ähnlichem Verhalten abzuschrecken und erneute Straffälligkeit zu verhindern.

Der Basisindikator für den Umfang der Gefängnisbevölkerung ist in jedem Land die Zahl der Gefängnisinsassen (einschließlich Untersuchungshäftlinge) je 100000 Einwohner. Die Daten über die Gefängnisbevölkerung lassen sich auch nach geographischen Merkmalen und Rechtsstatus aufschlüsseln. Die hier dargelegten Indikatoren wurden vom International Centre for Prison Studies zusammengetragen (www.prisonstudies.org).

In den vergangenen 15 Jahren hat die Gefängnisbevölkerung in den meisten OECD-Ländern kontinuierlich zugenommen. Im Durchschnitt der 30 OECD-Länder ist die Inhaftierungsrate von 100 Personen je 100000 Einwohner der Gesamtbevölkerung Anfang der neunziger Jahre auf etwa 130 Personen Mitte 2000 gestiegen (Abb. CO2.1). Am höchsten ist die Inhaftierungsrate in den Vereinigten Staaten, wo über 700 Personen je 100000 Einwohner 2005 in Gefängnissen einsaßen. Sie ist drei- bis viermal höher als im Land mit dem zweithöchsten Niveau (Polen) und hat über den in Abbildung CO2.1 dargelegten Zeitraum rasch zugenommen. Ein solcher Anstieg hat auch in den meisten anderen OECDLändern stattgefunden. Seit 1992 hat sich die Gefängnisbevölkerung in den Niederlanden, Mexiko, Japan, der Tschechischen Republik, Luxemburg, Spanien und dem Vereinigten Königreich mehr als verdoppelt, während sie offenbar nur in Kanada, Island und Korea rückläufig war.

In der Zusammensetzung der Gefängnisbevölkerung gibt es zwischen den Ländern große Unterschiede. Im Durchschnitt ist jeder vierte Inhaftierte in Untersuchungshaft, in der Türkei sowie in Mexiko und Luxemburg ist der Anteil dieser Kategorie an der Gefängnisbevölkerung aber wesentlich größer (Tabelle CO2.2). Frauen und Jugendliche (unter 18 Jahren) machen im Durchschnitt 5\% bzw. 2\% der Gefängnisbevölkerung aus - im Falle der Minderjährigen kann dies aber durch die spezifischen Sanktionsformen bedingt sein, die in den einzelnen OECD-Ländern Anwendung finden (Formen, die möglicherweise außerhalb des hier verwendeten statistischen Rahmens liegen). Ein erheblich größerer Anteil der Gefängnisbevölkerung besteht aus Ausländern (durchschnittlich nahezu 20\% aller Inhaftierten), wobei dieser Anteil in Luxemburg, der Schweiz wie auch Australien, Österreich, Belgien und Griechenland 40\% überschreitet.

In mehreren Ländern hat die rasche Zunahme der Gefängnisbevölkerung die Aufnahmekapazität der bestehenden Gefängniseinrichtungen überschritten. Die Belegungsquote liegt in über der Hälfte der OECD-Länder bei über $100 \%$ und in Griechenland, Ungarn, Italien, Spanien und Mexiko bei über 125\%. Eine derartige Überfüllung der Gefängnisse nährt Gewalt und Auflehnung gegen die entsprechenden Einrichtungen.
Den Strafvollzugssystemen in den einzelnen OECDLändern fehlt es generell an statistischen Instrumenten zur Analyse der individuellen Biographien, die einige Personen in das Verbrechen und in die Haft führen, wie auch der Effizienz des Systems zur Förderung ihrer Resozialisierung. Und dennoch konnte in mehreren Studien der enge Zusammenhang zwischen Inhaftierung auf der einen Seite und extremer Armut und Marginalisierung auf der anderen Seite dokumentiert werden. Die Inhaftierung betrifft in erster Linie Personen mit wenig sozialen Kontakten, die Familienzerfall, schulisches Scheitern und Gewalt erlebt haben. Gleichzeitig scheint die Inhaftierung (bestenfalls) begrenzte Auswirkungen auf die Resozialisierung ehemaliger Häftlinge gehabt zu haben: In Frankreich werden laut Kensey und Tournier (2004) etwa drei Viertel der wegen Einbruch verurteilten Täter innerhalb von fünf Jahren rückfällig, während aus nationalen Statistiken für die Vereinigten Staaten hervorgeht, dass zwei Drittel aller aus Gefängnishaft entlassenen Personen (über 630000 Personen pro Jahr) innerhalb von drei Jahren wieder festgenommen werden. Wegen der Gefahr, dass wiederholte Inhaftierungen die soziale Ausgrenzung verstärken können, haben einige Länder eine Reihe (begrenzter) Schritte zur Förderung der Wiedereingliederung dieser Personen in den Arbeitsmarkt ergriffen. In der Form kann es sich bei diesen Schritten um Interventionen öffentlicher Arbeitsverwaltungen, mit denen Inhaftierten die Rückkehr in den Arbeitsmarkt erleichtert wird, oder aber um stärker strukturierte Programme handeln (die häufig lokal umgesetzt werden), in denen vorberufliche Ausbildung, kurzfristige Übergangsbeschäftigungen sowie Vermittlungsdienste für Vollzeitarbeitsplätze miteinander kombiniert werden (CEO, 2006).

Statusindikatoren: Vertrauen in die politischen Institutionen (CO6), Lebenszufriedenheit (CO7), Arbeitslosigkeit (SS2).

Reaktionsindikatoren: Öffentliche Sozialausgaben (EQ5). 


\section{CO2.1 Ein größerer Teil der OECD-Bevölkerung befindet sich in Haft}

Inhaftierungsrate je 100000 Einw ohner
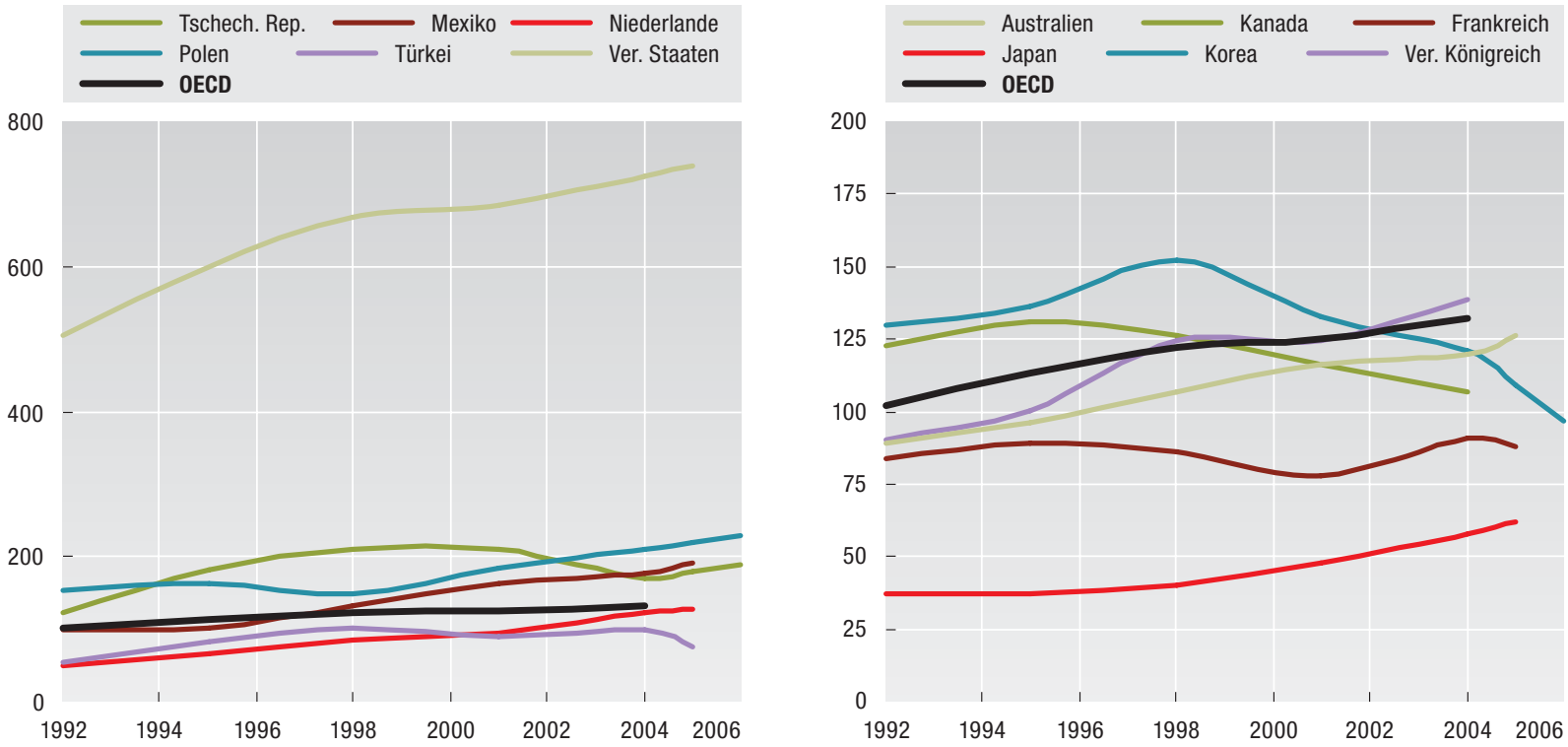

CO2.2 Große Unterschiede in der Zusammensetzung der Gefängnisbevölkerung in den OECD-Ländern

Inhaftierungsrate und Zusammensetzung, Belegungsniveau, letztes verfügbares Jahr

\begin{tabular}{|c|c|c|c|c|c|c|c|}
\hline & \multirow[b]{2}{*}{ Jahr } & \multirow{2}{*}{$\begin{array}{l}\text { Inhaftierungsrate } \\
\text { (je } 100000 \\
\text { Enwohner) }\end{array}$} & \multicolumn{4}{|c|}{ Zusammensetzung der Gefängnisbevölkerung (in \%) } & \multirow{2}{*}{$\begin{array}{l}\text { Belegungs- } \\
\text { niveau (in \%) }\end{array}$} \\
\hline & & & $\begin{array}{l}\text { Untersuchungs- } \\
\text { häftlinge }\end{array}$ & $\begin{array}{c}\text { Frauen } \\
\text { und Mädchen }\end{array}$ & $\begin{array}{l}\text { Jugendliche } \\
\text { (unter } 18 \text { Jahren) }\end{array}$ & Ausländer & \\
\hline Australien & 2005 & 126 & 20 & 7 & 0 & 17 & 106 \\
\hline Belgien & 2005 & 90 & 38 & 4 & 1 & 41 & 114 \\
\hline Dänemark & 2005 & 77 & 25 & 5 & 1 & 18 & 95 \\
\hline Deutschland & 2005 & 97 & 19 & 5 & 4 & 28 & 100 \\
\hline Finnland & 2006 & 75 & 12 & 6 & 0 & 8 & 112 \\
\hline Frankreich & 2005 & 88 & 36 & 4 & 1 & 21 & 110 \\
\hline Giechenland & 2005 & 90 & 28 & 6 & 7 & 42 & 179 \\
\hline Irland & 2004 & 85 & 16 & 3 & 2 & 9 & 95 \\
\hline Island & 2004 & 39 & 8 & 6 & 0 & 6 & - \\
\hline Italien & 2004 & 97 & 36 & 5 & 1 & 32 & 132 \\
\hline Japan & 2005 & 62 & 15 & 6 & 0 & 8 & 106 \\
\hline Kanada & 2004 & 107 & 30 & 5 & 6 & - & 90 \\
\hline Korea & 2006 & 97 & 29 & 5 & 1 & 1 & 100 \\
\hline Luxemburg & 2005 & 143 & 46 & 4 & 2 & 73 & - \\
\hline Mexiko & 2005 & 191 & 42 & 5 & - & 1 & 126 \\
\hline Neuseeland & 2006 & 189 & 16 & 6 & 1 & 9 & 102 \\
\hline Niederlande & 2005 & 127 & 31 & 9 & 1 & 33 & 98 \\
\hline Norwegen & 2005 & 68 & 19 & 5 & 0 & 18 & 97 \\
\hline Österreich & 2005 & 108 & 23 & 5 & 2 & 45 & 107 \\
\hline Polen & 2006 & 228 & 16 & 3 & 1 & 1 & 121 \\
\hline Portugal & 2006 & 123 & 23 & 7 & 2 & 17 & 105 \\
\hline Schweden & 2005 & 78 & 20 & 5 & 0 & 26 & 103 \\
\hline Schweiz & 2005 & 83 & 39 & 5 & 1 & 71 & 93 \\
\hline Slowak. Rep. & 2005 & 169 & 32 & 4 & 3 & 3 & - \\
\hline Spanien & 2006 & 143 & 23 & 8 & 0 & 30 & 130 \\
\hline Tschech. Rep. & 2006 & 189 & 14 & 5 & 1 & 8 & 104 \\
\hline Türkei & 2005 & 76 & 48 & 3 & 3 & 2 & - \\
\hline Ungarn & 2005 & 163 & 25 & 6 & 3 & 4 & 145 \\
\hline Ver. Königreich & 2006 & 143 & 18 & 6 & 3 & 11 & 109 \\
\hline Ver. Staaten & 2005 & 738 & 21 & 9 & 0 & 6 & 108 \\
\hline OECD & & 136 & 26 & 5 & 2 & 20 & 111 \\
\hline
\end{tabular}

Source: World Prison Brief, International Centre for Prison Studies, School of Law, King's College, University of London, Vereinigtes Königreich (www.prisonstudies.org).

StatLink 2 http://dx.doi.org/10.1787/613855171184

Weitere Informationen —Kensey, A. und P. Tournier (2004), "La récidive des sortants de prisons", Cahiers de démographie pénitentiaire, März. CEO (2006), The Power of Work, Center for Employment Opportunities Comprehensive Prisoner Reentry Program, New York, März. 


\section{Definition und Messung}

Die Suiziddaten in diesem Beitrag basieren auf amtlichen Statistiken, die alljährlich Informationen über die "Todesursachen" bei allen Sterbefällen liefern, wie in OECD (2006) dargelegt. Zur Bereinigung um den Effekt von Abweichungen in den Altersstrukturen zwischen den Ländern und im Zeitverlauf sind diese Suizidraten anhand der OECD-Bevölkerungsstruktur im Jahr 1980 standardisiert und je 100000 Einwohner ausgedrückt. Die Weltgesundheitsorganisation definiert den "Suizid" als eine Handlung, die eine Person in voller Kenntnis und in Erwartung des tödlichen Ausgangs selbst plant und ausführt. Die internationale Vergleichbarkeit der Suiziddaten wird durch eine Reihe von Faktoren beeinflusst, darunter die vom Standesbeamten zur Feststellung der Selbsttötungsabsicht der Person herangezogenen Kriterien, die für die Ausstellung der Sterbeurkunde verantwortliche Person, die Häufigkeit gerichtsmedizinischer Untersuchungen, die Beachtung der Schweigepflicht in Bezug auf die Todesursachen. All diese Faktoren werden vom kulturellen und religiösen Kontext jedes Landes beeinflusst.

Es lässt sich nur schwer beurteilen, welchen Effekt diese Faktoren auf die Ländervergleiche haben. Ein Konzept beruht auf der Untersuchung von Todesfällen, die eine Selbsttötungsabsicht von Personen verdecken können, wie beispielsweise Tod aus unbekannter Ursache, Tod durch Unfall sowie Tod durch Gewalteinwirkung, Fälle, in denen die Absicht der Person unbekannt ist. Auf der Basis dieses Ansatzes kommen Jougla et al. (2002) zu der Schlussfolgerung, dass die französischen Statistiken die tatsächliche Zahl der Suizide um etwa $20 \%$ zu niedrig ausweisen, wenngleich dies nur geringfügige Auswirkungen auf die Entwicklung der Daten im Zeitverlauf, die Merkmale der Suizidtäter und die Rangfolge der Regionen hat.

Die vorsätzliche Selbsttötung ist nicht nur Ausdruck eines persönlichen Zusammenbruchs, sondern auch einer Verschlechterung des sozialen Umfelds, in dem die Personen leben. Der Suizid kann viele Ursachen haben: Er erfolgt häufiger in Krisenzeiten in Verbindung mit Scheidung, Alkohol- und Drogenmissbrauch, Arbeitslosigkeit sowie in Fällen klinischer Depression oder bei sonstigen Formen psychischer Erkrankungen. Obwohl diese Faktoren häufig bei suizidgefährdeten Personen anzutreffen sind, hängt ihr Effekt auch von unterschiedlichen Faktoren auf gesellschaftlicher Ebene ab, die die Widerstandsfähigkeit des Einzelnen gegenüber externen Ereignissen entweder stärken oder schwächen.

Die durchschnittliche Suizidrate ist in den OECD-Ländern seit dem Höchststand Ende der achtziger Jahre geringfügig, aber stetig gesunken (Abb. CO3.1). Während dieser Rückgang bei beiden Geschlechtern zu beobachten ist, bleibt der Suizid dennoch ein hauptsächlich männliches Phänomen: So ist die Wahrscheinlichkeit der Selbsttötung unter Männern doppelt so hoch wie unter Frauen (Abb. CO3.2), wenngleich Frauen häufiger Selbstmordversuche begehen. Die Suizidfrequenz hängt auch vom Alter der Person ab, obwohl die altersbedingten Unterschiede im Laufe der Zeit geringer geworden sind. Im Allgemeinen sind die Suizidraten unter älteren Menschen in den vergangenen zwei Jahrzehnten deutlich gesunken, während bei den jüngeren Kohorten kaum Fortschritte verzeichnet wurden. In Finnland, Neuseeland, Irland und Island liegt die Suizidneigung der unter 25-Jährigen über dem nationalen Durchschnitt - die entsprechenden Raten sind in diesen Ländern seit 1980 drastisch gestiegen -, während dieselbe Bevölkerungsgruppe in Südeuropa und Mexiko Suizidgefahren weniger stark ausgesetzt ist.

Die durchschnittlichen Suizidraten in den 23 OECDLändern verdecken große Unterschiede. So reichen die Suizidraten von 5 Personen oder weniger je 100000 Einwohner in den meisten Mittelmeerländern bis zu über 20 Personen je 100000 Einwohner in Ungarn, Japan, Belgien bzw. Finnland. Die unterschiedlichen Suizidraten zwischen OECDLändern hängen nicht mit dem Pro-Kopf-Einkommen (Abb. CO3.3) und auch nur schwach mit dem selbst angegebenen Niveau der Lebenszufriedenheit zusammen (Abb. CO3.4). Empirische Studien sind zu dem Ergebnis gekommen, dass es diesel ben Faktoren sind, die die Unterschiede zwischen den Ländern bei der subjektiv empfundenen Lebenszufriedenheit und bei den Suizidraten erklären, wobei in den 50 untersuchten Ländern nahezu 80\% der Varianz durch Differenzen in der Häufigkeit von Scheidungen, Arbeitslosigkeit, Qualität der Regierungsführung, religiösen Überzeugungen, Vertrauen in andere Menschen und Mitgliedschaft in nichtreligiösen Vereinigungen bedingt ist (Helliwell, 2004).

Statusindikatoren: Lebenszufriedenheit (CO7), Lebenserwartung (HE1).

Reaktionsindikatoren: Gesundheitsausgaben (HE2). 
CO3.1 Niedrigere Suizidraten in den meisten OECD-Ländern in den vergangenen zwanzig Jahren Suizide je 100000 Einw ohner, 1960-2004

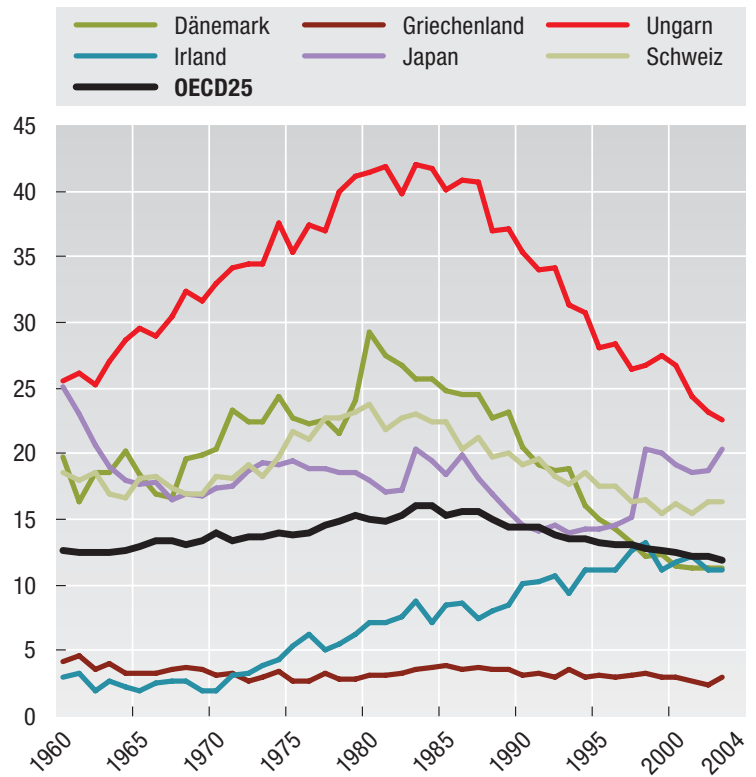

\section{CO3.3 Geringer Zusammenhang zwischen Suizidraten und Pro-Kopf-Einkommen in OECD-Ländern Suizidraten und Pro-Kopf-BIP, $2004^{1}$}

\section{Suizidraten}

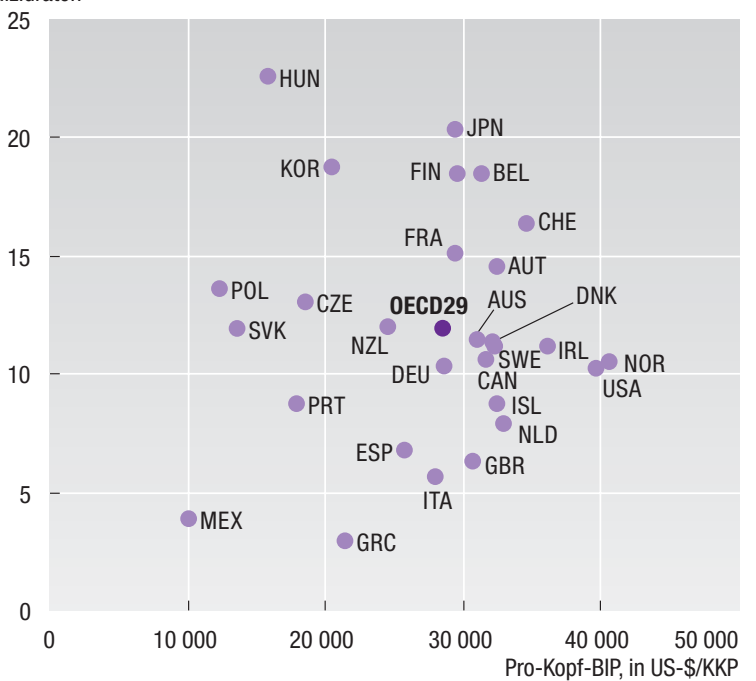

1. 2003 für Griechenland, Island, Japan, Norwegen, Polen, Portugal, Spanien und Ungarn; 2002 für Australien, Frankreich, Irland, ItaIien, Kanada, Korea, Schweden, Schweiz, Slowakische Republik, Vereinigtes Königreich und Vereinigte Staaten; 2001 für Dänemark und Neuseeland; 1997 für Belgien; 1995 für Mexiko.

Quelle: OECD (2006), OECD-Gesundheitsdaten, CD-ROM, Paris (vgl. auch www.oecd.org/health/healthdata); und Annual National Accounts.

\section{CO3.2 Höhere Suizidraten unter Männern als unter Frauen}

Suizide je 100000 Einw ohner nach Ländern und Geschlecht, 2004 oder letztes verfügbares Jahr

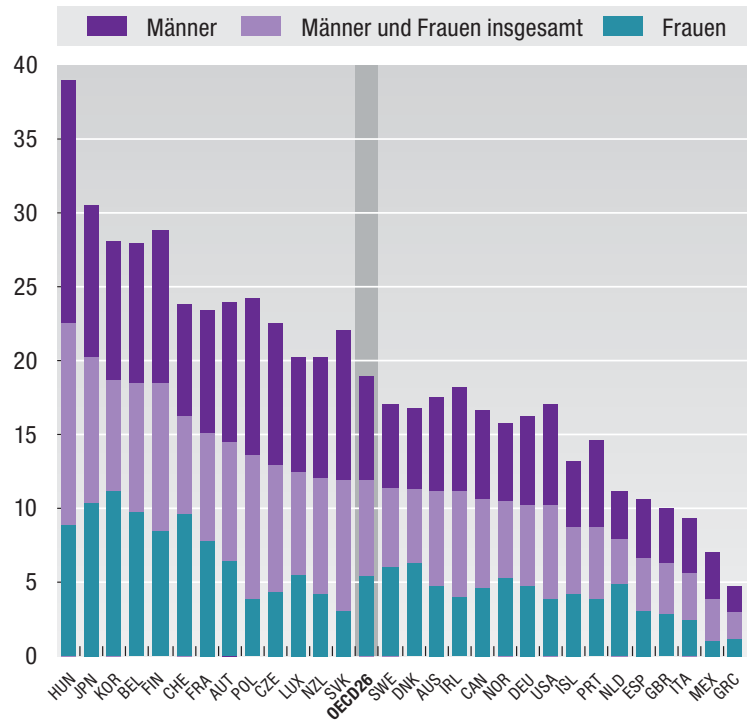

\section{CO3.4 Niedrigere Suizidraten in OECD-Ländern} mit höherem subjektiven Wohlbefinden Suizidraten und mittlere Lebenszufriedenheit ${ }^{1}$ Suizidraten

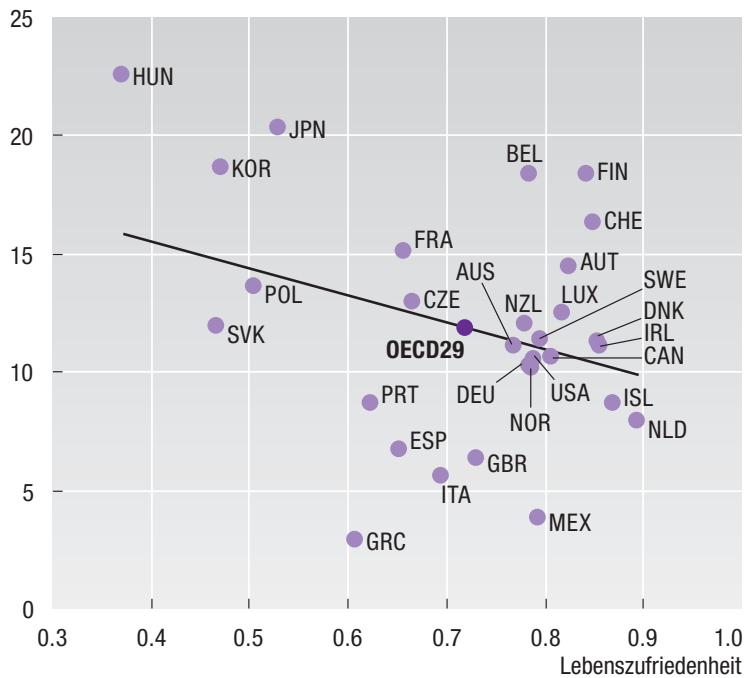

1. Die Suizidraten beziehen sich auf unterschiedliche Jahre, entsprechend zu Anmerkung 1 zu Abb. CO3.3. Die Daten zur Lebenszufriedenheit basieren auf der Erhebungswelle 1999/2001 des World Values Survey für alle Länder, außer Neuseeland und Schweiz, wo sie auf die Welle 1994-1995 zurückgehen.

Quelle: OECD (2006), OECD-Gesundheitsdaten, CD-ROM, Paris (vgl. auch www.oecd.org/health/healthdata) und Berechnungen aus dem World Values Survey.

S tatLink 2 http://dx.doi.org/10.1787/867063480631

Weitere Informationen — Baudelot, C. und R. Establet (2006), Suicide - l'envers de notre monde, Seuil, Paris. — Helliwell, J. (2004), "Well-Being and Social Capital: Does Suicide Pose a Puzzle?", NBER Working Paper, No. 10896, Boston. Jougla, E., F. Pequignot, J.L. Chappert, F. Rossolin, A. Le Toullec und G. Pavillon (2002), “La qualité des données de mortalité sur le suicide”, Revue d'épidémiologie et santé publique, Paris. 


\section{Definition und Messung}

Arbeitsunfälle sind unvorhergesehene und manchmal heftige Ereignisse, die während der Arbeit eintreten und für den Beschäftigten zu gesundheitlichen Schäden bzw. zum Tod führen. Internationale Vergleiche der Arbeitsunfälle gestalten sich auf Grund der Unterschiede in der Datenerfassung - in manchen Fällen werden in die Statistiken nur so genannte "entschädigte" Unfälle am Arbeitsplatz ab einem bestimmten Schweregrad aufgenommen, kleinere Verletzungen bleiben unberücksichtigt - und bei den Datenquellen (Versicherungsgesellschaften, Sozialversicherungsdaten, Gewerbeaufsicht, Unternehmens- und Sondererhebungen) schwierig. Die Vergleichbarkeit hat sich seit der Verabschiedung der ILO-Resolution von 1998 zu Statistiken über Verletzungen infolge von Arbeitsunfällen jedoch verbessert, in der Normen für die Datensammlung und -darstellung festgelegt werden. Die Resolution empfiehlt die Erfassung aller Arbeitsunfalldaten, die in einem bestimmten Referenzzeitraum (in der Regel ein Jahr) Ausfallzeiten von mindestens einem Tag zur Folge haben (unter Ausklammerung des Unfalltages).

Die hier darstellten Daten wurden von Eurostat anhand eines harmonisierten Fragebogens kompiliert, der sich auf die EU15-Länder und Norwegen erstreckt, und auf andere OECD-Länder ausgedehnt, für die in der ILO-Datenbank über Arbeitsstatistiken (Laborsta) Daten verfügbar sind. Die Meldung von Arbeitsunfällen ohne Todesfolge beschränkt sich in den europäischen Ländern und in Japan auf Verletzungen, die zu Abwesenheiten vom Arbeitsplatz von mehr als drei Tagen führen, in Australien sind es sechs oder mehr Tage und in anderen Ländern ein Tag oder mehrere Tage; hingegen bleiben in allen Ländern Fehlzeiten unberücksichtigt, die nur eine Reduzierung der Arbeitsstunden und kein eigentliches Fernbleiben vom Arbeitsplatz zur Folge haben. Die Frequenz der Arbeitsunfälle mit und ohne Todesfolge wird ausgedrückt als die Zahl der Arbeitsunfälle in 12 aufeinander folgenden Monaten je 100000 Arbeitskräfte. Der Schweregrad der am Arbeitsplatz erlittenen Verletzungen wird anhand der Zahl der durch Arbeitsunfälle je 100000 Arbeitskräfte verlorenen Arbeitstage gemessen. Bei den Daten für einige Länder sind Unfälle, die Selbstständige und Arbeitskräfte kleiner Unternehmen betreffen, möglicherweise ausgenommen. In einigen Fällen beziehen sich die Daten eher auf entschädigte als auf gemeldete Verletzungen und geben das Verhältnis Arbeitsunfälle/Versicherte und nicht die Relation Arbeitsunfälle/Gesamtzahl der Arbeitskräfte wieder.

Arbeitsunfälle sind der offensichtlichste Ausdruck für die Gefahren bezahlter Beschäftigung. Die meisten Arbeitsunfälle enden nicht tödlich. Im Jahr 2003 war die Zahl der tödlichen Arbeitsunfälle in der Türkei, Korea und Mexiko am höchsten und im Vereinigten Königreich sowie in Schweden am niedrigsten (Tabelle CO4.1), und ihre Zahl ist in allen Ländern, für die Daten verfügbar sind, seit 1995 zurückgegangen. Arbeitsunfälle ohne Todesfolge sind geläufiger, ihre Zahl reicht von 1200 je 100000 Arbeitskräfte in den Niederlanden bis zu 6500 in Spanien und scheint in allen Ländern, außer Spanien, ebenfalls zurückgegangen zu sein (Abb. CO4.2). In den Vereinigten Staaten könnte dieser Rückgang durch eine Verschärfung der Versicherungskonditionen bedingt gewesen sein, die Arbeitgebern mehr Anreize bieten, kleinere Unfälle entweder weniger häufig zu melden oder verletzten Arbeitnehmern eine reduzierte Stundenzahl anzubieten: Infolgedessen ist die Gesamtzahl der Arbeitsunfälle (6 200 je 100000 Arbeitskräfte im Jahr 2000) wegen der Vielzahl der Arbeitsunfälle, die entweder nur eine eingeschränkte Berufsausübung (1 200) oder überhaupt keine Abwesenheit vom Arbeitsplatz zur Folge haben (3 200 Fälle; Ruser, 2002) mehr als dreimal so hoch wie die Zahl der in Tabelle CO4.1 ausgewiesenen Arbeitsunfälle, die zu einer mehrtägigen Abwesenheit vom Arbeitsplatz führen.

Sowohl die tödlichen als auch die nicht tödlich endenden Arbeitsunfälle sind sehr stark auf die Bereiche Landwirtschaft, bestimmte Zweige des Verarbeitenden Gewerbes, das Baugewerbe und den Straßenverkehr konzentriert. Da es sich bei den Arbeitskräften in diesen Sektoren hauptsächlich um erwachsene Männer handelt, entfällt über die Hälfte aller tödlichen Unfälle auf Arbeitskräfte in der Altersgruppe 45-54 Jahre (und über die Hälfte aller nicht tödlich endenden Unfälle auf Arbeitskräfte zwischen 25 und 44 Jahren). In Europa ging mit dem seit 1995 beobachteten Rückgang der tödlichen Unfälle um 25\% eine steigende Konzentration der Arbeitsunfälle auf ältere Arbeitskräfte einher. Beschäftigungsverlagerungen zwischen Sektoren erklären zwar einen Teil des Rückgangs der Arbeitsunfälle, dieser hat in den Vereinigten Staaten aber in allen Branchen stattgefunden (zumindest bei den Unfällen ohne Todesfolge).

Die Durchschnittsdauer der arbeitsunfallbedingten Abwesenheit vom Arbeitsplatz betrug im Jahr 2001 weniger als sechs Tage, in Spanien hingegen nahezu acht Tage. In den Vereinigten Staaten lag der Mittelwert der auf Grund von Arbeitsunfällen verlorenen Arbeitstage bei sechs Tagen je Vollzeitarbeitskraft. In allen Ländern ist die Dauer dieser Abwesenheiten im Verarbeitenden Gewerbe wie auch in der Landwirtschaft und im Baugewerbe besonders hoch.

Arbeitsunfälle sind mit bedeutenden wirtschaftlichen Kosten für die Arbeitskräfte, Firmen und Gemeinwesen verbunden. Trotz der schwierigen Quantifizierung reichten die Schätzungen dieser Kosten - im Verein mit den Kosten für Berufskrankheiten - in mehreren OECD-Ländern von 0,4-4\% des BIP (EASHW, 1998). Eine Reduzierung der Zahl der Arbeitsunfälle setzt ein Arbeitsumfeld voraus, in dem die Beschäftigten über die entsprechenden Qualifikationen und Schulung zur Ausübung der von ihnen am Arbeitsplatz geforderten Aufgaben verfügen und Firmen Anreize geboten werden, das Auftreten von Arbeitsunfällen so weit wie möglich zu vermeiden.

Statusindikatoren: Krankheitsbedingte Abwesenheit vom Arbeitsplatz (HE4).

Reaktionsindikatoren: Öffentliche Sozialausgaben (EQ5), Gesundheitsausgaben (HE2). 
CO4.1 Tödliche Arbeitsunfälle sind in der Türkei, Korea und Mexiko häufiger als in anderen OECD-Ländern Unfälle mit und ohne Todesfolge im Jahr 2003 je 100000 A rbeitskräfte, verlorene A rbeitstage je betroffenen Beschäftigten im Jahr 2001

\begin{tabular}{|c|c|c|c|c|c|c|c|}
\hline & \multicolumn{3}{|c|}{ Arbeitsunfälle } & \multicolumn{4}{|c|}{ Nicht tödliche Arbeitsunfälle nach Branchen } \\
\hline & Tödlich & Nicht tödlich & $\begin{array}{l}\text { Verlorene Tage } \\
\text { je Betroffenen }\end{array}$ & Landwirtschaft & $\begin{array}{l}\text { Verarbeitendes } \\
\text { Gewerbe }\end{array}$ & Baugewerbe & Straßenverkehr \\
\hline & \multicolumn{7}{|c|}{ Entschädigte Arbeitsunfälle } \\
\hline Australien & 2.0 & 1230 & 6.0 & 2561 & 2070 & 2201 & 2056 \\
\hline Finnland & 2.7 & 2847 & 5.7 & 5226 & 3339 & 5908 & 3534 \\
\hline Deutschland & 3.5 & 3674 & 4.3 & 12160 & 3432 & 7029 & 3702 \\
\hline Luxemburg & 3.6 & 5033 & $\ldots$ & 9795 & 4887 & 10812 & 4415 \\
\hline Belgien & 3.9 & 3456 & 5.7 & 5387 & 3572 & 6398 & 3898 \\
\hline Griechenland & 3.9 & 2090 & $\ldots$ & 1265 & 3226 & 4519 & 1820 \\
\hline Neuseeland & 5.2 & 1605 & $\ldots$ & $\ldots$ & .. & $\ldots$ & .. \\
\hline Frankreich & 5.4 & 4689 & 5.9 & 4778 & 4232 & 10066 & 6123 \\
\hline Kanada & 6.1 & 2227 & 5.7 & 2212 & 3914 & 3428 & 2650 \\
\hline \multirow[t]{2}{*}{ Korea (2001) } & 15.5 & . & . & . & . & $\cdots$ & . \\
\hline & \multicolumn{7}{|c|}{ Gemeldete Arbeitsunfälle } \\
\hline Ver. Königreich & 1.0 & 1614 & $\ldots$ & 2139 & 1519 & 2493 & 1868 \\
\hline Schweden & 1.6 & 1252 & 5.3 & 1355 & 1717 & 2090 & 1583 \\
\hline Niederlande & 1.8 & 1188 & . & . & . & . & . \\
\hline Dänemark & 2.4 & 2443 & . & 1284 & 4141 & 3773 & 2991 \\
\hline Japan & 3.1 & 233 & $\ldots$ & 1028 & 287 & 584 & 440 \\
\hline Norwegen & 3.1 & 3325 & $\ldots$ & 3161 & 5563 & 5835 & 4448 \\
\hline Ungarn & 3.4 & 656 & $\ldots$ & 748 & 1235 & 469 & 960 \\
\hline Irland & 3.9 & 1262 & $\ldots$ & $\ldots$ & . & . & $\ldots$ \\
\hline Tschech. Rep. & 4.5 & 1872 & 6.4 & 3947 & 3256 & 3429 & 1966 \\
\hline Slowak. Rep. & 4.7 & 801 & 5.1 & 2720 & 1601 & 2049 & 882 \\
\hline Polen & 4.9 & $\ldots$ & 5.0 & . & . & . & . \\
\hline Italien & 5.6 & 3267 & $\ldots$ & $\ldots$ & .. & $\ldots$ & $\ldots$ \\
\hline Spanien & 6.0 & 6520 & 7.7 & 2401 & 8820 & 13651 & 6526 \\
\hline Österreich & 6.6 & 2629 & $\ldots$ & $\ldots$ & . & $\ldots$ & $\ldots$ \\
\hline Ver. Staaten & 8.0 & 1626 & 6.0 & . & . & .. & .. \\
\hline Portugal & 8.4 & 4054 & $\ldots$ & 880 & 5773 & 6851 & 3624 \\
\hline Mexiko & 12.0 & 2968 & . & $\ldots$ & $\ldots$ & $\ldots$ & $\ldots$ \\
\hline Türkei (2001) & 20.6 & . & $\ldots$ & . & $\ldots$ & .. & .. \\
\hline
\end{tabular}

.: Nicht verfügbar.

Anmerkung: In jedem Teil der Tabelle sind die Länder in aufsteigender Reihenfolge der tödlichen Unfälle angeordnet. Die Daten zur Frequenz der tödlichen und nicht tödlichen Arbeitsunfälle in den EU15-Ländern und Norwegen werden anhand der Beschäftigungsstruktur (nach Industriezweig) der EU15-Länder gewichtet.

Quelle: ILO-Laborsta-Datenbank, Eurostat Referenzdatenbank New Cronos sowie die BLS Website über tödliche Arbeitsunfälle und Berufsunfälle (www.bls.gov/iif).

\section{CO4.2 Nicht tödliche Arbeitsunfälle sind in den meisten Ländern rückläufig} Nicht tödliche Arbeitsunfälle je 100000 A rbeitskräfte, 1995-2003

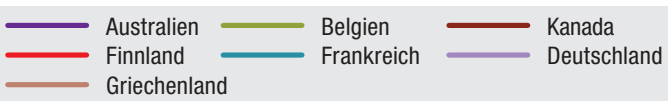

10000 Griechenland

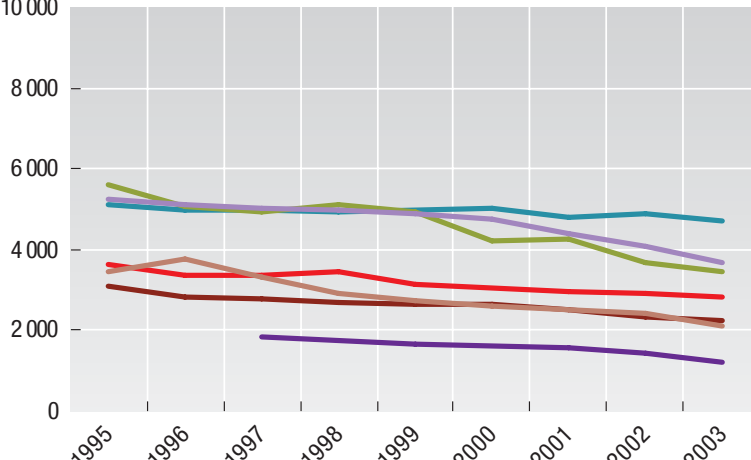

Quelle: ILO-Laborsta-Datenbank; Eurostat-Referenzdatenbank New Cronos.
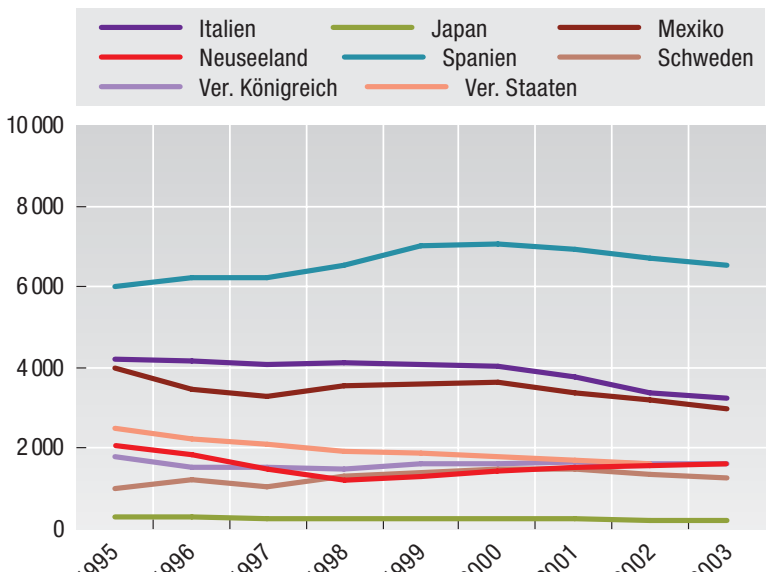

StatLink $2 \quad$ http://dx.doi.org/10.1787/552432835786

Weitere Informationen — European Agency for Safety and Health at Work - EASHW (1998), Economic Impact of Occupational Safety and health in Member States of the European Union, Bilbao. Ruser, J. (2002), M easuring Workplace Safety and Health: general considerations and the US case, US Bureau of Labor Statistics, UNECE-Eurostat-ILO seminar on Measurement of the quality of employment, Genf, Mai. 


\section{Definition und Messung}

Streiks sind ein Ausdruck von Arbeitskonflikten. Auf der Internationalen ILO-Konferenz der Arbeitsstatistiker wurden Streiks definiert als vorübergehende Arbeitsniederlegung bzw. Schließung einer Arbeitsstätte auf Initiative einer oder mehrerer Gruppen von Arbeitnehmern oder Arbeitgebern, um Forderungen durchzusetzen bzw. abzuwehren und Beschwerden auszudrücken oder andere Arbeitnehmer bzw. Arbeitgeber in ihren Forderungen oder Beschwerden zu unterstützen. Der umfassendste Indikator von Arbeitskonflikten ist der Anteil der auf Grund von Streiks verlorenen Arbeitsstunden, dieser ist aber nur für einige wenige Länder verfügbar. Daher wird hier als Hauptindikator das Verhältnis zwischen der Anzahl der infolge von Streiks verlorenen Arbeitstage und der Gesamtzahl der Arbeitskräfte verwendet.

Die internationale Vergleichbarkeit von Streikdaten wird durch Unterschiede in der Definition und Messung erschwert. Viele Länder schließen kleinere Arbeitsniederlegungen aus den amtlichen Statistiken aus und legen je nach Zahl der teilnehmenden Arbeitskräfte und/oder Zahl der verlorenen Arbeitstage unterschiedliche Schwellenwerte zu Grunde. Unberücksichtigt bleiben in einigen Ländern auch Arbeitsniederlegungen in bestimmten Industriezweigen, wie beispielsweise im öffentlichen Dienst, oder auch bestimmte Streikformen sowie politische oder ungenehmigte Streiks. Umgekehrt sind in den Statistiken anderer Länder auch indirekt betroffene Arbeitskräfte (d.h. jene, die auf Grund der Streiktätigkeit anderer am Arbeitsplatz selbst nicht arbeiten können) oder durch ausbleibende Materiallieferungen auf Grund von Streiks in Zulieferunternehmen verursachte Arbeitsniederlegungen aufgenommen. Im Allgemeinen sind Aktionen, bei denen es nicht zu einer vollständigen Arbeitsniederlegung kommt, wie "Bummelstreiks", stille oder sonstige Proteste am Arbeitsplatz nicht inbegriffen. Die OECD trägt in regelmäßigen Abständen Streikdaten aus internationalen und nationalen Quellen zusammen, um die Arbeitsmarkttrends in den Mitgliedsländern aufzuzeigen.

Da die Streiktätigkeit von Jahr zu Jahr erheblich abweichen kann, sind über mehrere aufeinander folgende Jahre berechnete Durchschnittswerte der verlässlichste Indikator für die Beobachtung der Trendentwicklungen bei Arbeitskonflikten. Abbildung CO5.1 enthält Fünfjahresdurchschnitte der Streikquote, d.h. der Gesamtzahl der auf Grund von Streiks je 1000 Arbeitskräfte verlorenen Arbeitstage in zwei Zeiträumen - zum einen 1980-1984 und zum anderen 2000-2004 - für 25 OECD-Länder (Teil A). In beiden Zeiträumen verzeichneten Island, Italien, Kanada und Spanien die höchste Zahl der in Arbeitskonflikten verlorenen Arbeitstage je Arbeitskraft, während diese Zahl in Deutschland, Japan, den Niederlanden und der Schweiz am niedrigsten war.

Im OECD-Raum hat sich die Streikquote seit Anfang der achtziger Jahre alle zehn Jahre in etwa halbiert. Der seit Anfang der achtziger Jahre beobachtete Rückgang war in Australien, Finnland, Irland, Island, Italien, Kanada, Spanien und dem Vereinigten Königreich besonders stark. Veränderungen in der Beschäftigungsstruktur nach Industriezweigen mit einer Verlagerung in Richtung der Dienstleistungssektoren erklären einen Teil dieses Rückgangs, da die Streikquoten in der Industrie (Bergbau, Verarbeitendes Gewerbe, Strom, Versorgungsunternehmen und Baugewerbe) generell doppelt so hoch sind wie im Dienstleistungssektor (mit Ausnahme des Verkehrssektors). Dennoch sind die Streikquoten in den meisten OECD-Ländern in den vergangenen zehn Jahren sowohl im Industrie- als auch im Dienstleistungssektor gesunken (Beardsmore, 2006).

Die Streikquote lässt sich nach dem Anteil der an Arbeitskonflikten beteiligten Arbeitskräfte und der Durchschnittsdauer dieser Arbeitskonflikte je Arbeitskraft ausdrücken (Teile C und D). Die Durchschnittsdauer der Arbeitskonflikte lag im Zeitraum 2000-2004 bei 71/2 Tagen, wobei nur sieben Länder je Arbeitskraft mehr als zehn Tage an Arbeitsniederlegungen verzeichneten. Die Türkei und die Vereinigten Staaten meldeten mehr Streiktage, allerdings mit einer vergleichsweise geringen Zahl von Arbeitskräften und Arbeitsniederlegungen (in den Daten für die Vereinigten Staaten bleiben indessen Streiks mit einer Beteiligung von weniger als 10000 Arbeitskräften unberücksichtigt). In allen OECD-Ländern nahm parallel zum Rückgang der Streikquoten die Zahl der betroffenen
Arbeitskräfte ab, während die Streikdauer in Island, Korea, Norwegen und den Vereinigten Staaten zunahm. In jüngster Zeit ist die Zahl der Arbeitskonflikte nur in Dänemark gestiegen, was sich aber nicht in einer höheren Streikquote niederschlug (Teil B).

Zwischen Streiks und sonstigen Merkmalen des Systems der Arbeitsbeziehungen besteht kein einfacher Zusammenhang. In Abbildung CO5.2 werden die Streikquoten der jüngeren Vergangenheit und der Grad der Tarifgebundenheit (d.h. der Anteil der Arbeitnehmer, deren Gehalts- und Arbeitsbedingungen im Rahmen eines Tarifvertrags zwischen Arbeitgebern und Gewerkschaften geregelt sind) sowie die Streikquoten und der gewerkschaftliche Organisationsgrad (d.h. der Anteil der Arbeitnehmer, die einer Gewerkschaft angehören ) im Rahmen eines Koordinatensystems dargestellt. Sehr niedrig sind die Streikquoten sowohl in Ländern mit hohem Grad der Tarifgebundenheit (z.B. Finnland und Schweden) als auch in Ländern, in denen dies nur für wenige Arbeitskräfte gilt (z.B. Japan, wenngleich in den Daten für dieses Land wilde Streiks und Streiks von weniger als einem Tag unberücksichtigt bleiben). Ferner sind die Streikquoten in Ländern mit hohem gewerkschaftlichen Organisationsgrad (Dänemark) und Ländern mit einem Organisationsgrad von unter $20 \%$ (z.B. Vereinigte Staaten) verhältnismäßig niedrig, mit einem schwachen Trend zu Gunsten höherer Streikquoten in Ländern mit geringer Gewerkschaftsdichte. Jüngste Forschungsergebnisse legen den Schluss nahe, dass die Qualität der Arbeitgeber-Arbeitnehmer-Beziehungen Einfluss auf die Arbeitsmarktergebnisse hat. Addison und Texeira (2006) kommen beispielsweise zu dem Ergebnis, dass nach Bereinigung um eine Palette sonstiger Faktoren OECD-Länder, in denen die Arbeitsbeziehungen (gemessen anhand der Streikquoten) weniger konfliktreich sind, die Arbeitslosenquote niedriger ist als in anderen Ländern.

Statusindikatoren: Wahlbeteiligung (CO1), Vertrauen in die politischen Institutionen (CO6), Lebenszufriedenheit (CO7), Beschäftigung (SS1). 


\section{CO5.1 Messgrößen von Streiks in OECD-Ländern}

A. Streikquoten: verlorene Arbeitstage je 1000 abhängig Beschäftige ${ }^{1}$ - $1980-1984$ 2000-2004

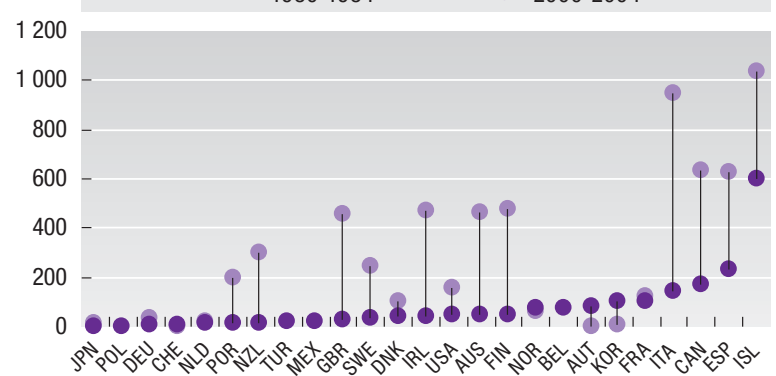

C. Durchschnittsdauer der Arbeitsniederlegungen (in Tagen je beteiligter Arbeitskraft) ${ }^{3}$

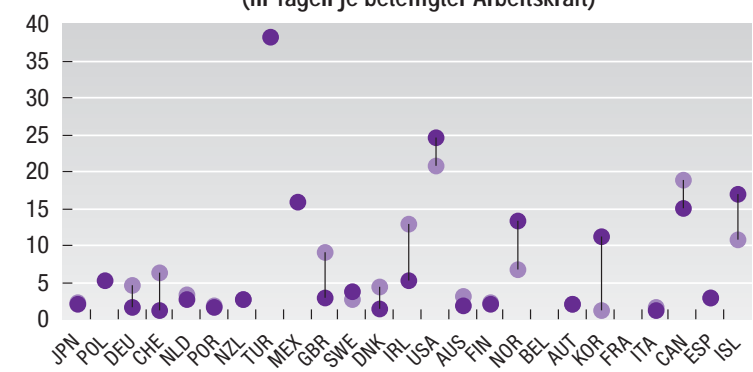

B. Intensität der Streiks (je 100000 abhängig Beschäftigte) ${ }^{2}$

1980-1984

- 2000-2004

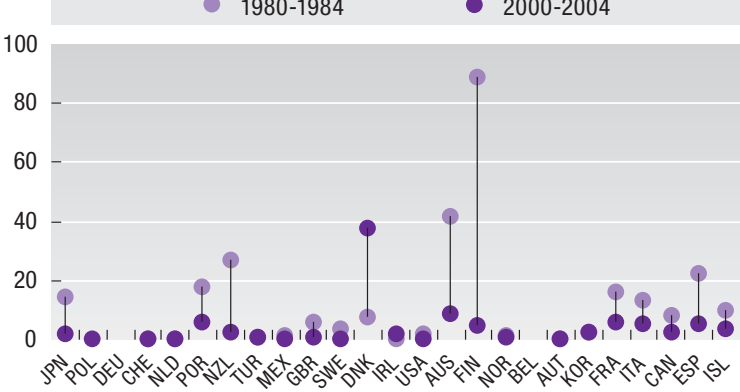

D. Beteiligte Arbeitskräfte (je 1000 abhängig Beschäftigte) ${ }^{4}$

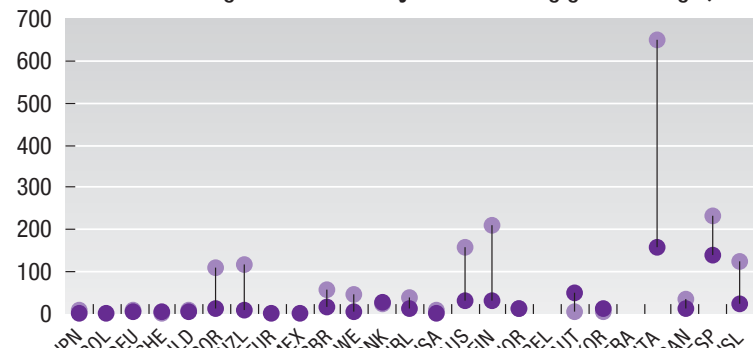

Anmerkung: Die Länder sind in aufsteigender Reihenfolge der je 1000 Arbeitnehmer verlorenen Arbeitstage angeordnet (Streikquoten).

1. Zahl der Tage mit Arbeitsniederlegungen je 1000 abhängig Beschäftigte.

2. Zahl der Arbeitsniederlegungen je 100000 abhängig Beschäftigte.

3. Zahl der Tage mit Arbeitsniederlegungen je beteiligter Arbeitskraft.

4. Zahl der beteiligten Arbeitskräfte je 1000 abhängig Beschäftigte.

Quelle: OECD-Daten abgeleitet von ILO Laborsta, Eurostat New Cronos und NSO-Websites. Die Angaben zu den zivilen abhängig Beschäftigten stammen aus OECD Labour Force Statistics.

\section{C05.2 Keine Korrelation zwischen Streiks und Tarifgebundenheit bzw. Streiks und Gewerkschaftsdichte}

\section{A. Streiks und Grad der Tarifgebundenheit}

Grad der Tarifgebundenheit, in \%

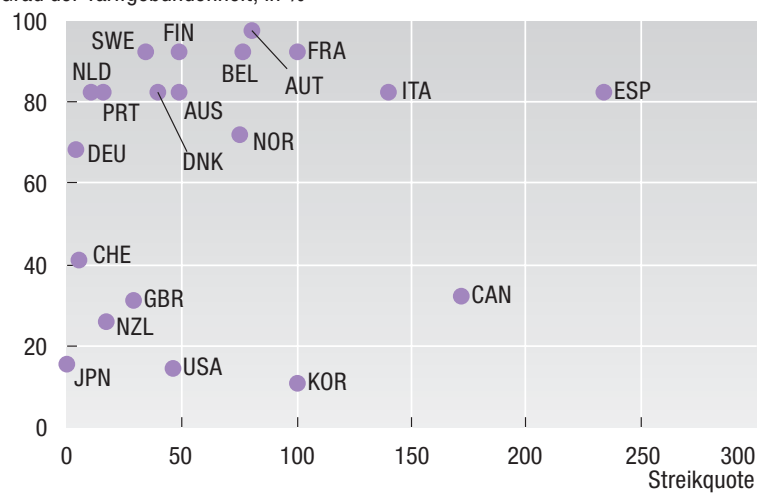

B. Streiks und Gewerkschaftsdichte

Gewerkschaftsdichte, in \%

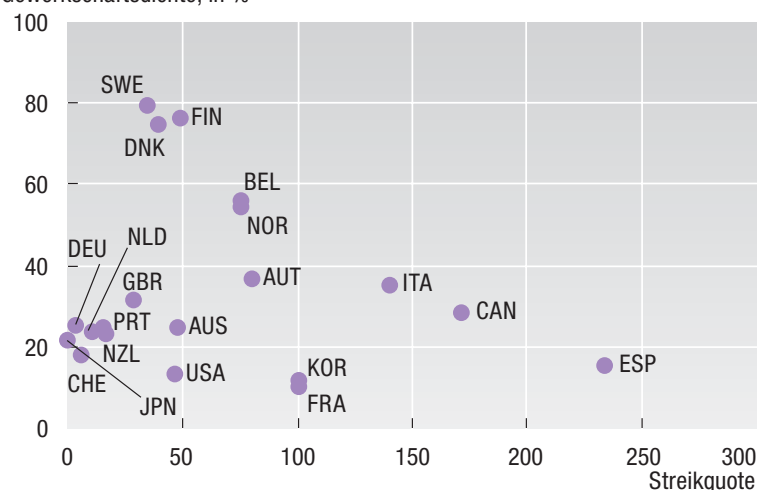

Anmerkung: Alle Daten sind in Prozent der zivilen abhängig Beschäftigten ausgedrückt.

Quelle: Die Angaben zu Gewerkschaftsdichte und Tarifgebundenheit sind Tabelle 3.3 aus OECD (2004), OECD Employment Outlook, Paris, entnommen; für die Daten zu Streikquoten vgl. Abbildung CO6.1.

Weitere Informationen —Addison, J.T. und P. Texeira (2006), “Does the Quality of Industrial Relations Matter for the Macro-economy? A cross-country analysis using strikes data", IZA Discussion Paper, No. 1968, Februar. Beardsmore, R. (2006), "International Comparisons of Labour Disputes in 2004", Labour Market Trends, Special feature, United Kingdom Office for National Statistics. — ILO (2005), Yearbook of Labour Statistics 2005, Internationale Arbeitsorganisation, Genf. 


\section{Definition und Messung}

Der Indikator des Vertrauens in die politischen Institutionen misst, inwieweit die Bürger hohes Vertrauen in die politischen Institutionen (Regierung und Parlament) und die öffentliche Verwaltung des Landes haben, in dem sie leben. Die Daten zu diesen Variablen sind der Erhebungswelle 1999-2004 des World Values Survey entnommen, in der die Bürger nach dem Grad ihres Vertrauens in eine Reihe von Organisationen gefragt wurden; die Antworten wurden in vier Kategorien eingeteilt (sehr viel Vertrauen, recht viel, nicht sehr viel und überhaupt kein Vertrauen). Die weiter unten dargelegten Indikatoren beziehen sich auf den Prozentsatz aller Befragten, die angaben, "sehr viel" oder "recht viel" Vertrauen in Regierung, Parlament und öffentlichen Dienst zu haben. Die Datenvergleichbarkeit zwischen den Ländern kann durch den kleinen Stichprobenumfang und sonstige Erhebungsmerkmale beeinträchtigt werden.

Der vorliegende Abschnitt enthält ferner Daten über die Trendentwicklung der Zufriedenheit des Einzelnen mit der Funktionsweise der Demokratie in seinem Land. Die Daten sind verschiedenen Erhebungen entnommen, wie in OECD (2005) beschrieben. Der hier verwendete Indikator bezieht sich auf den Prozentsatz der Befragten, die sich mit dem demokratischen Prozess entweder "sehr" oder "recht" zufrieden erklären.

Das Vertrauen in den öffentlichen Dienst und den politischen Prozess ist für die Stabilität der Gesellschaft und das Funktionieren der Demokratie in jedem Land von entscheidender Bedeutung; ferner ist es ein grundlegender Faktor für die Bereitschaft der Menschen zur Zusammenarbeit bei der Erreichung gemeinsamer Ziele und Finanzierung öffentlicher Güter (Meikle-Yaw, 2006). Zwischen den OECD-Ländern gibt es in Bezug auf das Ausmaß des Bürgervertrauens in verschiedene öffentliche Einrichtungen große Unterschiede (Abb. CO6.1). Im Durchschnitt gaben 38\% der Personen in 24 OECD-Ländern zu Beginn dieses Jahrzehnts an, großes Vertrauen in ihr Parlament zu haben, wobei der Anteil in Island, Luxemburg, Norwegen, den Niederlanden, Schweden und Spanien größer und in Korea, der Tschechischen Republik, Japan, Mexiko und Australien kleiner war. Ein geringfügig kleinerer Anteil der Befragten gab in 17 OECD-Ländern an, großes Vertrauen in die Regierung zu haben, mit niedrigeren Anteilen in Neuseeland, Deutschland, Australien, der Tschechischen Republik und Korea und höheren Anteilen in Schweden, der Slowakischen Republik, Spanien, der Schweiz und der Türkei.

Auch in der Einschätzung der Funktionsweise des öffentlichen Dienstes, mit dem die einzelnen Bürger im Alltag am stärksten konfrontiert sind, bestehen zwischen den Ländern bedeutende Unterschiede. Etwa 44\% der OECDBürger geben an, großes Vertrauen in den öffentlichen Dienst zu haben, wobei die jeweiligen Anteile in Griechenland, Japan, Mexiko und der Tschechischen Republik niedriger und in der Türkei, Korea, Luxemburg und Irland höher sind. In der Mehrzahl der OECD-Länder geben die Bürger an, mehr Vertrauen in den öffentlichen Dienst als in die Regierung zu haben, die größten Ausnahmen bilden diesbezüglich die Tschechische Republik, Mexiko, Polen und Neuseeland. Der öffentliche Dienst spielt in der Gesellschaft eine Schlüsselrolle: Haben Bürger wenig Vertrauen in den öffentlichen Dienst, kann dies zu Unzufriedenheit mit dem kollektiven Handeln und dem gesamten politischen Prozess führen. Schlecht konzipierte Reformen können auch das Vertrauen der Bürger in den öffentlichen Dienst untergraben. Auch wenn es keine direkte Verknüpfung zwischen der Leistung des öffentlichen Dienstes und der subjektiven Evaluierung der Bürger zu geben scheint, wird in OECD (2005) veranschaulicht, dass ein größeres Vertrauen in den öffentlichen Dienst mit stärkerem Vertrauen in das Parlament Hand in Hand geht.

Schwieriger ist die Beurteilung der Frage, wie sich das Vertrauen der Bürger in die politischen Institutionen im Lauf der Zeit verändert hat. Die Rolle der Modernisierung stand häufig im Mittelpunkt der Diskussion, und einige Autoren legen den Schluss nahe, dass die Verbreitung von Informationen und die Anhebung des Bildungsniveaus der Bürger zu einem Vertrauensverlust in staatliche Einrichtungen geführt haben könnten. Jedoch bietet die bei der Bürgerzufriedenheit generell beobachtete Trendentwicklung wenig Belege für einen allgemeinen Rückgang der Zufriedenheit mit der Demokratie (Abb. CO6.2). Zwischen den Ländern bestehen aber bedeutende Unterschiede: Eine geringere Zufriedenheit mit der Funktionsweise der Demokratie wird in Japan und seit kurzem auch in den Niederlanden beobachtet; noch geringer ist die Zufriedenheit mit der Demokratie in Italien, wo sich die Lage aber bessert; ein höheres Niveau an Zufriedenheit mit der Demokratie wurde in Dänemark und anderen nordischen Ländern verzeichnet. Generell fordern die Bürger häufig mehr Mitwirkung und Beteiligung an öffentlichen Angelegenheiten. Als Reaktion auf diese Forderungen haben die Regierungen in mehreren OECD-Ländern Reformen in die Wege geleitet, um die öffentlichen Dienste offener, transparenter und stärker kundenorientiert zu gestalten.

Statusindikatoren: Wahlbeteiligung (CO1), Lebenszufriedenheit (CO7). 
CO6.1 Weniger als die Hälfte der OECD-Bürger gibt großes Vertrauen in verschiedene öffentliche Institutionen an Anteil der Befragten, die Anfang dieses Jahrzehnts angaben, großes Vertrauen in verschiedene Einrichtungen zu haben
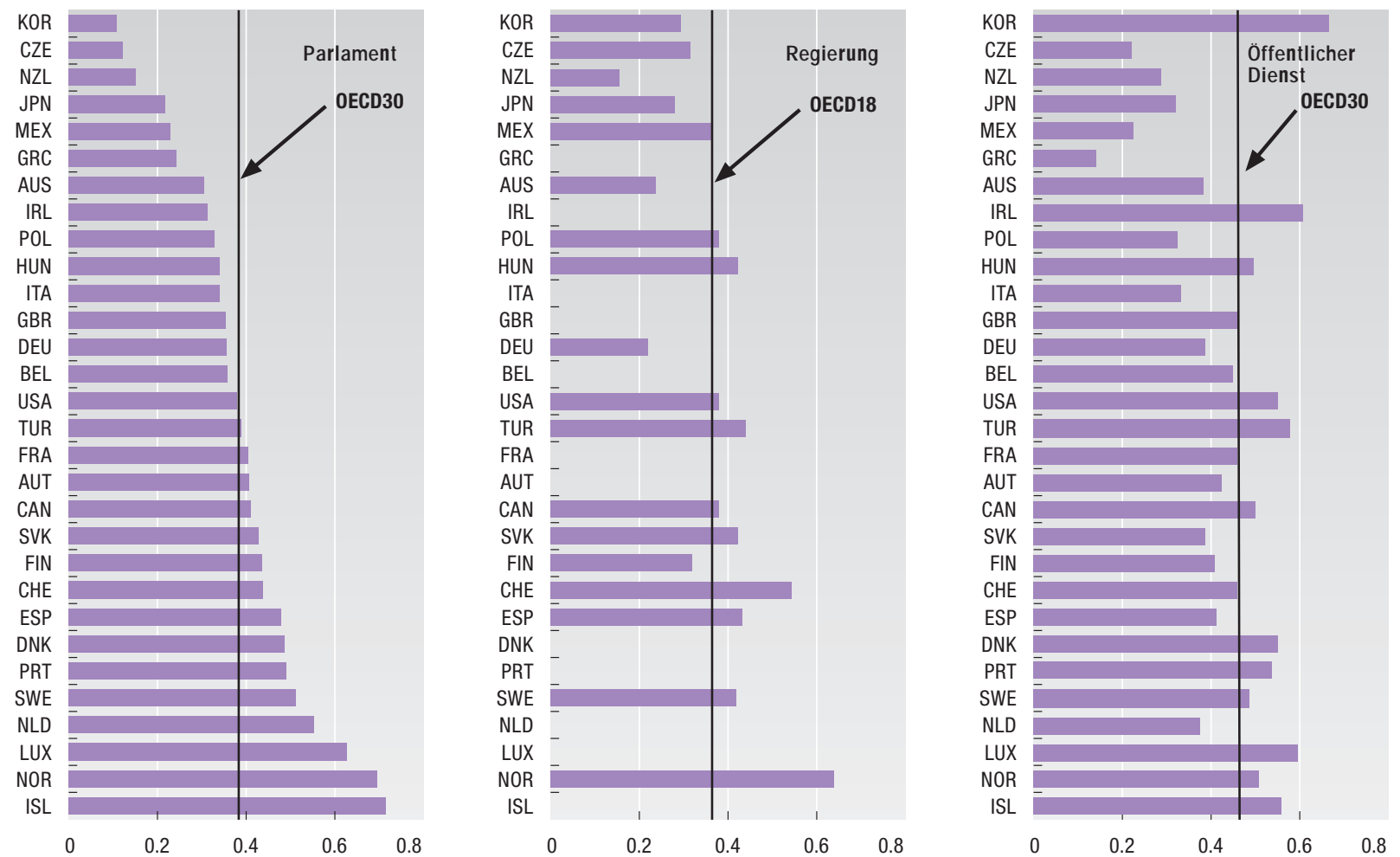

Quelle: Die Daten sind der Erhebungswelle 1999-2004 des World Values Survey entnommen. Für Australien, Norwegen, Neuseeland und die Schweiz beziehen sich die Daten auf die Erhebungswelle 1994-1999.

C06.2 Kein allgemeiner Rückgang der Zufriedenheit mit der Demokratie

Prozentsatz der Befragten, die mit dem demokratischen Prozess "sehr" oder "recht" zufrieden waren, 1975-2005
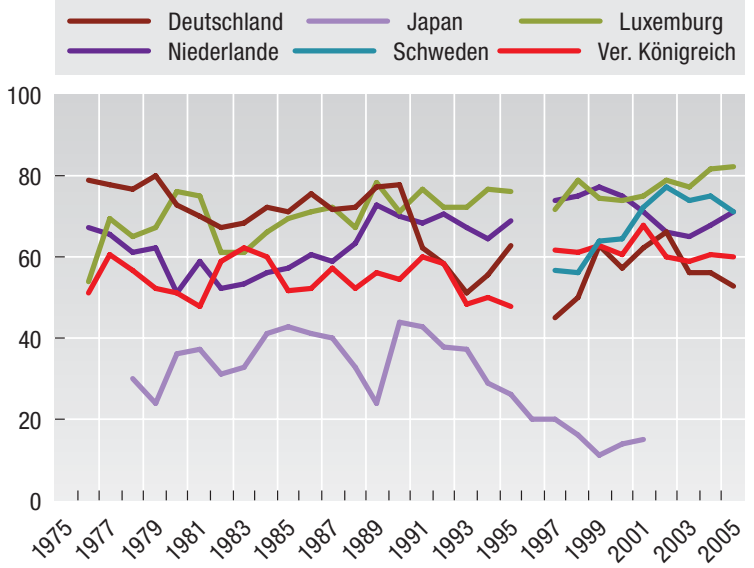
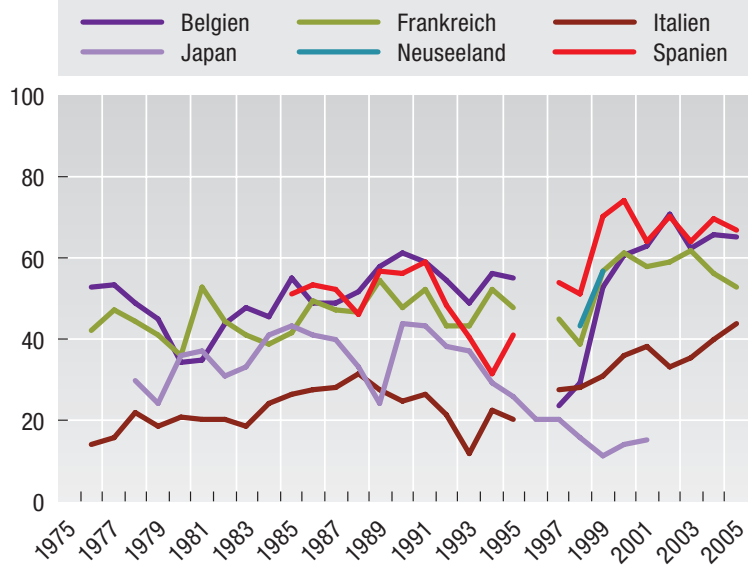

Quelle: Die Daten für die europäischen Länder sind Eurobarometer-Erhebungen entnommen; die Daten für Japan stammen aus nationalen Quellen und beziehen sich auf den Prozentsatz der Befragten, die sich mit den Politikern zufrieden erklären. Wegen näherer Einzelheiten, vgl. OECD (2005), "Data on Trust in the Public Sector", Tagung auf Ministerebene des Ausschusses für öffentliche Verwaltung, 27.-28. November, Paris.

StatLink 2 http://dx.doi.org/10.1787/020765758801

Weitere Informationen a Meikle-Yaw, P.A. (2006), “Democracy Satisfaction: The Role of Social Capital and Civic Engagement in Local Communities", Department of Sociology, Anthropology, and Social Work, Mississippi State University. 


\section{Definition und Messung}

Subjektive Messgrößen der Lebenszufriedenheit geben an, bis zu welchem Grad eine Person ihre Lebensqualität insgesamt als positiv bewertet. Zusammengetragen werden die Daten im Rahmen von Erhebungen, in denen Personen gefragt werden, "wie zufrieden" sie mit ihrem Leben insgesamt (und in bestimmten Bereichen) sind, wobei die Befragten ihre Zufriedenheit auf einer Skala von 1 bis 10 bewerten (von der niedrigsten bis zur höchsten Zufriedenheit). Der in diesem Abschnitt verwendete Indikator entspricht dem Anteil der Befragten, der seine Lebenszufriedenheit auf dieser Skala mit mindestens 7 einstuft. Im Mittelpunkt der Analyse stehen die Unterschiede in der Beurteilung der Lebenszufriedenheit durch verschiedene Personengruppen (je nach Geschlecht, Alter, Bildungsniveau, Beschäftigungsstatus, Familienstand und Einkommen) und die Frage nach Korrelationen zwischen dem Durchschnittswert jedes Landes und einer Reihe sonstiger sozialer und wirtschaftlicher Aspekte.

Der hier verwendete Indikator der Lebenszufriedenheit ist den Ergebnissen der Erhebungswelle 1999-2004 des World Values Survey entnommen. Diese Erhebung sammelt Daten, die Vergleiche von Wertvorstellungen, Normen und Einstellungen in unterschiedlichen sozialen Bereichen ermöglichen. Konkret werden die Erhebungen in Interviews unter vier Augen mit Erwachsenen durchgeführt. Die in Abbildung C07.2 dargelegten Indikatoren des Vertrauens in das Parlament (der Anteil der Befragten, der angibt entweder "sehr viel" oder "recht viel Vertrauen" in das Parlament zu haben) und das Vertrauen in Menschen (der Anteil der Befragten, der der Meinung ist, dass "den meisten Menschen vertraut werden kann") wurden von Daten aus derselben Erhebung abgeleitet.

Die umfangreiche Literatur über das subjektive Wohlbefinden veranschaulicht, dass dieses unter Personen mit unterschiedlichen genetischen, Persönlichkeits- und wirtschaftlichen Merkmalen systematisch variiert. Tabelle C07.1 stellt Daten über die Lebenszufriedenheit nach Geschlecht, Alter, Familienstand, Schulbildung sowie (nach Eigenangaben) Einkommensniveau dar. Im Durchschnitt des OECD-Raums ist die Lebenszufriedenheit unter Männern geringfügig höher als unter Frauen, wenngleich in mehreren Ländern das Gegenteil der Fall ist (z.B. Finnland, Japan und Türkei); auch in Bezug auf das Alter sind kleine Unterschiede zu beobachten. Höher ist die durchschnittliche Lebenszufriedenheit unter den Älteren als unter den Jüngeren in elf OECD-Ländern, darunter Japan und Korea. Demgegenüber ist die Lebenszufriedenheit junger Menschen in Ungarn, Polen und der Slowakischen Republik im Durchschnitt $10 \%$ höher als die der älteren Bevölkerung.

Der hier verwendete Indikator legt den Schluss nahe, dass die Lebenszufriedenheit im Durchschnitt mit dem Bildungsniveau steigt. Das erklärt sich möglicherweise aus dem Effekt der Bildung auf andere Bereiche, die Einfluss auf die Lebenszufriedenheit haben (z.B. haben Personen mit höherem Bildungsabschluss häufiger eine bessere Gesundheit, ein höheres Einkommen und mehr soziale Interaktion). Die Lebenszufriedenheit schwankt noch stärker mit dem Familienstand. Im Durchschnitt ist die Lebenszufriedenheit verheirateter Personen um etwa 10 Punkte höher als die Lebenszufriedenheit geschiedener und verwitweter Personen, wobei der Unterschied gegenüber getrennt lebenden Personen noch größer ist. Alleinstehende bzw. nie verheiratet gewesene Personen sind mit ihrem Leben im Durchschnitt ebenfalls weniger zufrieden als verheiratete Personen (Deutschland, Polen, die Slowakische Republik und die Türkei bilden hier Ausnahmen). Auch nach sozioökonomischem Status (der in der Tabelle nicht aufgeführt ist) weichen die Werte für die Lebenszufriedenheit ab. Unter Arbeitslosen wird ein Niveau der Lebenszufriedenheit beobachtet, das etwa 20 Punkte unter dem der Erwerbstätigen liegt, da Arbeitslosigkeit nicht nur höheren finanziellen Stress zur Folge hat, sondern auch einen Verlust an Selbstwertgefühl, weniger soziale Kontakte und häufigeres Auftreten seelischer und geistiger Probleme. Umgekehrt sind die Unterschiede in der Lebenszufriedenheit zwischen Vollzeit- und Teilzeitkräften, Selbstständigen und Studenten generell gering. Schließlich sind Personen mit höherem Einkommen (in den drei oberen Dezilen der Verteilung) generell mit ihrem Leben zufriedener als Personen mit niedrigerem Einkommen, obgleich der Abstand proportional geringer ist als der Einkommensunterschied (Helliwell, 2002).

Die Lebenszufriedenheit hängt auch mit den Merkmalen der Gesellschaft zusammen, in der eine Person lebt. Die durchschnittliche Lebenszufriedenheit ist in den Ländern höher, in denen das Vertrauen in andere und auch in das Parlament hoch ist (Abbildung CO7.2, obere Teile; Bjornskov et al., 2005; Helliwell und Hang, 2006). Umgekehrt scheinen höhere Inflationsraten und längere Arbeitszeiten die Lebenszufriedenheit negativ zu beeinflussen (untere Teile).

Statusindikatoren: Vertrauen in die politischen Institutionen (CO6), Materielle Entbehrung (EQ1), Armutspersistenz (EQ7). 
C07.1 Die Lebenszufriedenheit variiert zwischen Personen mit unterschiedlichen Merkmalen Anteil der Befragten, die ein hohes Niveau der Lebenszufriedenheit angeben

\begin{tabular}{|c|c|c|c|c|c|c|c|c|c|c|c|c|c|c|c|c|}
\hline & \multicolumn{2}{|c|}{ Geschlecht } & \multicolumn{4}{|c|}{ Alter } & \multicolumn{3}{|c|}{ Schulbildung } & \multicolumn{4}{|c|}{ Familienstand } & \multicolumn{3}{|c|}{ Enkommen } \\
\hline & Männer & Frauen & $<25$ & $25-50$ & $51-64$ & $65+$ & Niedrig & Mittel & Hoch & Geschieden & Verheiratet & $\begin{array}{l}\text { Alleinstehend/ } \\
\text { noch nie } \\
\text { verheiratet }\end{array}$ & Verwitwet & Hoch & Mittel & Niedrig \\
\hline Belgien & 0.73 & 0.75 & 0.81 & 0.71 & 0.78 & 0.75 & 0.67 & 0.75 & 0.80 & 0.63 & 0.82 & 0.69 & 0.61 & 0.85 & 0.75 & 0.61 \\
\hline Dänemark & 0.85 & 0.81 & 0.85 & 0.85 & 0.82 & 0.78 & 0.82 & 0.88 & 0.84 & 0.72 & 0.87 & 0.83 & 0.76 & 0.92 & 0.87 & 0.75 \\
\hline Deutschland & 0.72 & 0.74 & 0.78 & 0.71 & 0.71 & 0.79 & 0.70 & 0.76 & 0.75 & 0.61 & 0.75 & 0.76 & 0.74 & 0.78 & 0.79 & 0.66 \\
\hline Finnland & 0.83 & 0.86 & 0.82 & 0.86 & 0.84 & 0.82 & 0.79 & 0.91 & 0.86 & 0.76 & 0.91 & 0.78 & 0.79 & 0.89 & 0.88 & 0.76 \\
\hline Frankreich & 0.67 & 0.64 & 0.72 & 0.66 & 0.61 & 0.65 & 0.63 & 0.67 & 0.70 & 0.58 & 0.72 & 0.64 & 0.53 & 0.78 & 0.64 & 0.54 \\
\hline Griechenland & 0.59 & 0.58 & 0.57 & 0.58 & 0.61 & 0.62 & 0.52 & 0.56 & 0.64 & 0.61 & 0.63 & 0.56 & 0.51 & 0.69 & 0.58 & 0.49 \\
\hline Irland & 0.86 & 0.83 & 0.82 & 0.84 & 0.83 & 0.87 & 0.82 & 0.85 & 0.88 & 0.82 & 0.90 & 0.80 & 0.85 & 0.90 & 0.84 & 0.80 \\
\hline Island & 0.85 & 0.86 & 0.84 & 0.89 & 0.82 & 0.79 & 0.81 & 0.87 & 0.95 & 0.69 & 0.91 & 0.85 & 0.81 & 0.93 & 0.89 & 0.77 \\
\hline Italien & 0.71 & 0.66 & 0.67 & 0.69 & 0.70 & 0.67 & 0.64 & 0.70 & 0.74 & 0.72 & 0.73 & 0.66 & 0.54 & 0.77 & 0.66 & 0.62 \\
\hline Japan & 0.50 & 0.53 & 0.50 & 0.47 & 0.54 & 0.59 & 0.45 & 0.50 & 0.62 & 0.44 & 0.55 & 0.42 & 0.58 & 0.65 & 0.48 & 0.46 \\
\hline Kanada & 0.78 & 0.79 & 0.85 & 0.75 & 0.81 & 0.80 & 0.78 & 0.77 & 0.83 & 0.69 & 0.85 & 0.70 & 0.80 & 0.85 & 0.82 & 0.70 \\
\hline Korea & 0.45 & 0.45 & 0.45 & 0.44 & 0.45 & 0.57 & 0.33 & 0.46 & 0.46 & 0.23 & 0.50 & 0.39 & 0.35 & 0.60 & 0.47 & 0.33 \\
\hline Luxemburg & 0.83 & 0.81 & 0.79 & 0.82 & 0.87 & 0.82 & 0.76 & 0.84 & 0.85 & 0.81 & 0.85 & 0.80 & 0.81 & 0.89 & 0.88 & 0.75 \\
\hline Mexiko & 0.79 & 0.81 & 0.82 & 0.78 & 0.79 & 0.87 & 0.78 & 0.80 & 0.85 & 0.77 & 0.82 & 0.80 & 0.78 & 0.81 & 0.80 & 0.76 \\
\hline Niederlande & 0.90 & 0.89 & 0.96 & 0.90 & 0.88 & 0.85 & 0.85 & 0.92 & 0.91 & 0.80 & 0.96 & 0.88 & 0.76 & 0.94 & 0.92 & 0.79 \\
\hline Österreich & 0.83 & 0.80 & 0.83 & 0.82 & 0.82 & 0.79 & 0.78 & 0.85 & 0.88 & 0.71 & 0.85 & 0.80 & 0.76 & 0.88 & 0.78 & 0.77 \\
\hline Polen & 0.47 & 0.49 & 0.63 & 0.48 & 0.43 & 0.46 & 0.43 & 0.49 & 0.61 & 0.32 & 0.50 & 0.56 & 0.36 & 0.60 & 0.53 & 0.38 \\
\hline Portugal & 0.66 & 0.65 & 0.70 & 0.69 & 0.60 & 0.62 & 0.53 & 0.74 & 0.83 & 0.65 & 0.69 & 0.63 & 0.53 & 0.77 & 0.60 & 0.43 \\
\hline Schweden & 0.80 & 0.78 & 0.77 & 0.79 & 0.80 & 0.80 & 0.78 & 0.78 & 0.82 & 0.72 & 0.87 & 0.71 & 0.63 & 0.87 & 0.83 & 0.70 \\
\hline Slowak. Rep. & 0.45 & 0.44 & 0.53 & 0.44 & 0.43 & 0.40 & 0.37 & 0.47 & 0.54 & 0.30 & 0.45 & 0.50 & 0.38 & 0.54 & 0.40 & 0.35 \\
\hline Spanien & 0.65 & 0.66 & 0.65 & 0.66 & 0.67 & 0.61 & 0.60 & 0.70 & 0.70 & 0.71 & 0.69 & 0.65 & 0.51 & 0.70 & 0.69 & 0.52 \\
\hline Tschech. Rep. & 0.67 & 0.65 & 0.64 & 0.69 & 0.60 & 0.66 & 0.59 & 0.69 & 0.76 & 0.54 & 0.71 & 0.65 & 0.52 & 0.75 & 0.66 & 0.57 \\
\hline Türkei & 0.33 & 0.42 & 0.41 & 0.34 & 0.38 & 0.39 & 0.37 & 0.35 & 0.38 & 0.25 & 0.38 & 0.38 & 0.35 & 0.49 & 0.32 & 0.29 \\
\hline Ungarn & 0.38 & 0.39 & 0.49 & 0.38 & 0.32 & 0.39 & 0.33 & 0.43 & 0.54 & 0.29 & 0.43 & 0.38 & 0.26 & 0.56 & 0.31 & 0.23 \\
\hline Ver. Königreich ${ }^{1}$ & 0.76 & 0.71 & 0.76 & 0.73 & 0.73 & 0.71 & 0.74 & 0.72 & 0.77 & 0.65 & 0.78 & 0.73 & 0.70 & 0.80 & 0.71 & 0.64 \\
\hline Ver. Staaten & 0.77 & 0.76 & 0.78 & 0.72 & 0.81 & 0.85 & 0.73 & 0.76 & 0.79 & 0.70 & 0.84 & 0.74 & 0.75 & 0.89 & 0.76 & 0.72 \\
\hline OECD26 & 0.69 & 0.68 & 0.71 & 0.68 & 0.68 & 0.69 & 0.64 & 0.70 & 0.74 & 0.60 & 0.73 & 0.66 & 0.61 & 0.77 & 0.69 & 0.59 \\
\hline
\end{tabular}

1. Großbritannien.

\section{CO7.2 Die durchschnittliche Lebenszufriedenheit in den OECD-Ländern hängt von einer Reihe nationaler Merkmale ab}

Lebenszufriedenheit

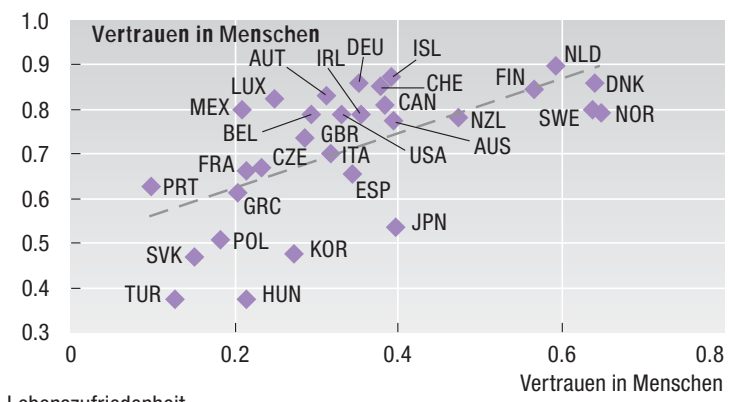

Lebenszufriedenheit

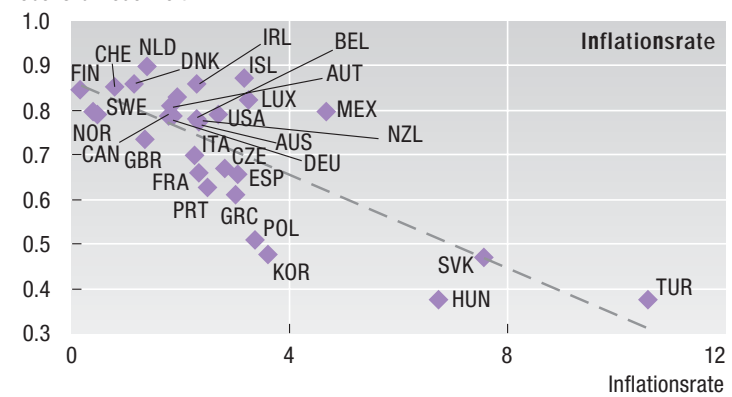

Lebenszufriedenheit

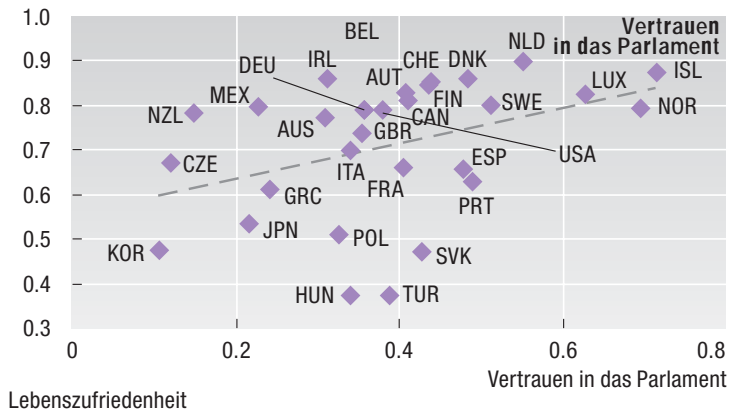

Lebenszufriedenheit

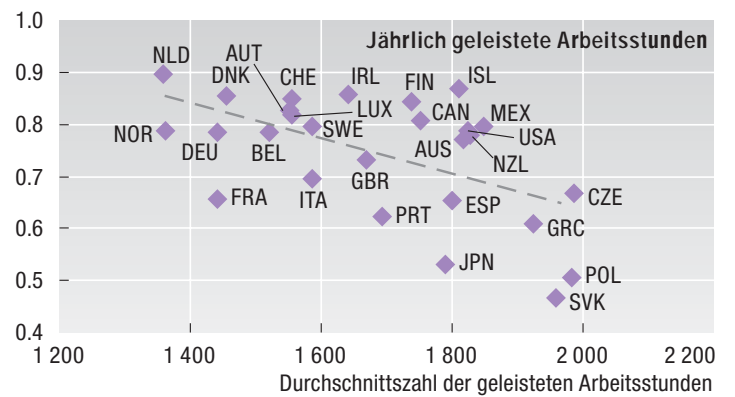

Quelle: Daten aus unterschiedlichen Erhebungswellen des World Values Survey und sonstige OECD-Daten. Die Daten zur Lebenszufriedenheit sind der Erhebungswelle 1999-2004 des World Values Survey entnommen, außer für Neuseeland und die Schweiz, wo sie sich auf die Erhebungswelle 1994-1999 beziehen.

StatLink $2 \quad$ http://dx.doi.org/10.1787/687418556206

Weitere Informationen — Bjornskov, C., A. Dreher und J. A.V. Fischer (2005), “Cross Country Determinants of Life Satisfaction”, Discussion Paper, No. 2005-19, Universität St. Gallen. "Helliwell, J.F. (2002), “How's Life? Combining Individual and national variables to explain subjective well-being", Working Paper, No. 11988, NBER, Boston. Helliwell, J.F. und H. Huang (2006), “How's your Government? International evidence linking good government and well-being", Working Paper, No. 11988, NBER, Boston. 
OECD PUBLICATIONS, 2, rue André-Pascal, 75775 PARIS CEDEX 16 PRINTED IN FRANCE

(81 2007025 P) ISBN 978-92-64-02955-2 - No. 554602007 